\title{
Directing Matter and Energy: Five Challenges for Science and the Imagination
}

A Report from the Basic Energy Sciences Advisory Committee 


\section{ON THE COVER:}

Fabrication of new crystalline materials with atomic-level control. To understand charge ordering and screening in mixed valence oxides, this oxide superlattice has been designed, synthesized, and then spectroscopically characterized at the atomic scale using scanning transmission electron microscopy. In the false color elastic images, white stripes are layers of $\mathrm{LaTiO}_{3}$; purple stripes are $\mathrm{SrTiO}_{3}$. Small images show individual atomic layers-in the topmost principal-component-filtered image, pink is lanthanum; light purple is strontium; and green is titanium.

(From "Artificial Charge-Modulation in Atomic-scale Perovskite Titanate Superlattices," A. Ohtomo, D. A. Muller, J. L. Grazul, H. Y. Hwang, Nature, 419, 378 (2002). Reprinted by permission from Macmillan Publishers Ltd: Nature.) 


\title{
Directing Matter and Energy: Five Challenges for Science and the Imagination
}

\author{
A Report from the \\ Basic Energy Sciences Advisory Committee
}

Chair:

John Hemminger (University of California, Irvine)

U.S. Department of Energy

December 20, 2007

Prepared by the BESAC Subcommittee on Grand Challenges for Basic Energy Sciences

Co-Chairs:

Graham Fleming (Lawrence Berkeley National Laboratory and University of California, Berkeley) Mark Ratner (Northwestern University) 



\section{BASIC ENERGY SCIENCES ADVISORY COMMITTEE}

\section{Chair:}

John Hemminger (University of California, Irvine)

Nora Berrah (Western Michigan University)

Sylvia Ceyer (Massachusetts Institute of Technology)

Sue Clark (Washington State University)

Peter Cummings (Vanderbilt University)

Frank DiSalvo (Cornell University)

Mostafa El-Sayed (Georgia Institute of Technology)

George Flynn (Columbia University)

Bruce Gates (University of California, Davis)

Laura Greene (University of Illinois at Urbana-Champaign)

Eric Isaacs (Argonne National Laboratory)

Anthony Johnson (University of Maryland)

Kate Kirby (Harvard-Smithsonian Center for Astrophysics)

Walter Kohn (University of California, Santa Barbara)

Gabrielle Long (Argonne National Laboratory)

William McCurdy, Jr. (Lawrence Berkeley National Laboratory)

Daniel Morse (University of California, Santa Barbara)

Martin Moskovits (University of California, Santa Barbara)

Ward Plummer (University of Tennessee)

John Richards (California Institute of Technology)

John Spence (Arizona State University)

Kathleen Taylor (General Motors, retired)

Mary Wirth (University of Arizona)

\section{Designated Federal Officer:}

Patricia M. Dehmer (U.S. Department of Energy, Associate Director of Science for Basic Energy Sciences)

\section{Committee Manager:}

Karen Talamini (U.S. Department of Energy, Office of Basic Energy Sciences) 


\section{SUBCOMMITTEE ON GRAND CHALLENGES FOR BASIC ENERGY SCIENCES}

\section{Co Chairs:}

Graham Fleming (Lawrence Berkeley National Laboratory and University of California, Berkeley)

Mark Ratner (Northwestern University)

Paul Alivisatos (Lawrence Berkeley National Laboratory and University of California, Berkeley)

Leon Balents (University of California, Santa Barbara)

David Bishop (Alcatel-Lucent Bell Labs)

Philip Bucksbaum (Stanford University and Stanford Linear Accelerator Center)

Jay Groves (Lawrence Berkeley National Laboratory and University of California, Berkeley)

John Hemminger [ex officio] (University of California, Irvine)*

Michael Hochella (Virginia Polytechnic Institute and State University)

Walter Kohn (University of California, Santa Barbara)**

Tobin Marks (Northwestern University)

Paul McEuen (Cornell University)

Joel Moore (Lawrence Berkeley National Laboratory and University of California, Berkeley)

Tom Moore (Arizona State University)

Cherry Murray (Lawrence Livermore National Laboratory)

Daniel Nocera (Massachusetts Institute of Technology)

Teri Odom (Northwestern University)

Julia Phillips (Sandia National Laboratories)

Pete Schultz (Scripps Research Institute and Genomics Institute of the Novartis Research Foundation)

Robert Silbey (Massachusetts Institute of Technology)

John Spence (Arizona State University)**

Stan Williams (Hewlett-Packard Laboratories)

Jun Ye (University of Colorado at Boulder and JILA)

\section{Subcommittee Manager:}

Tiffaney D. Dressen (Lawrence Berkeley National Laboratory)

* BESAC Chair

** BESAC Member 


\section{TABLE OF CONTENTS}

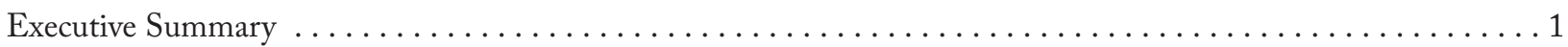

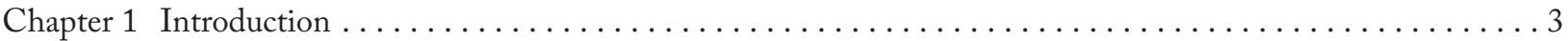

Chapter 2 Quantum Control of Electrons in Atoms, Molecules, and Materials:

Creating a New Language for the Behavior of Electrons $\ldots \ldots \ldots \ldots \ldots \ldots \ldots \ldots \ldots \ldots \ldots \ldots \ldots \ldots$

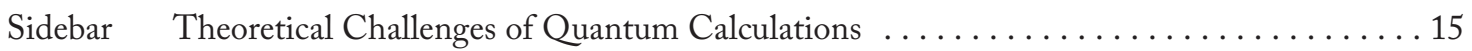

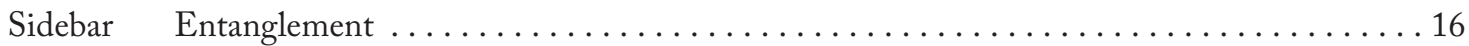

Sidebar Conical Intersections and Wavepacket Dynamics $\ldots \ldots \ldots \ldots \ldots \ldots \ldots \ldots \ldots \ldots \ldots \ldots$

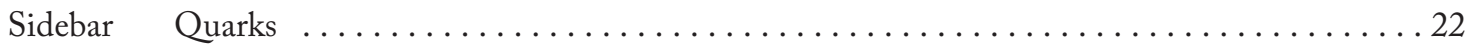

Sidebar Quantum Electron Scattering ................................ 25

Chapter 3 The Basic Architecture of Matter: Directed Assemblies, Structure, and Properties . . . . . . . . . . 29

Sidebar Nature's Answer to Fragile Building Blocks $\ldots \ldots \ldots \ldots \ldots \ldots \ldots \ldots \ldots \ldots \ldots \ldots \ldots \ldots$

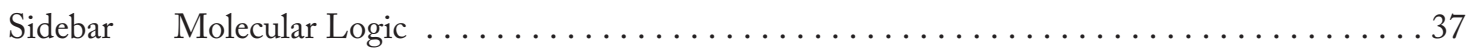

Sidebar $\quad$ Solar Fuels ......................................... 43

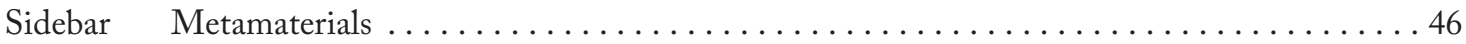

Chapter 4 Emergence of Collective Phenomena: Strongly Correlated Multiparticle Systems . . . . . . . . . . . 49

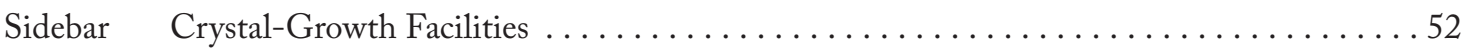

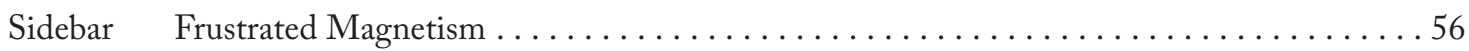

Sidebar High-Temperature Superconductivity .............................. 59

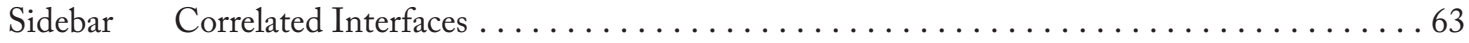

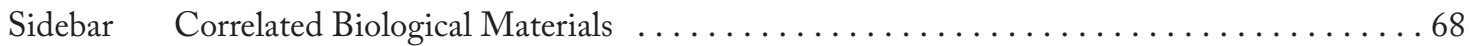

Chapter 5 Realizing the Dream of Nanoscience: Energy and Information on the Nanoscale . . . . . . . . 73

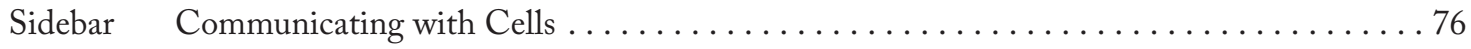

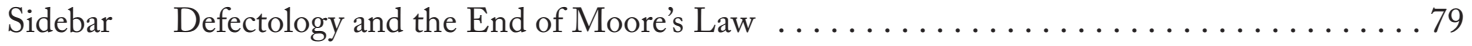

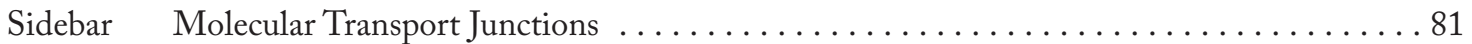

Sidebar $\quad$ Noise Can Be Useful . . . . . . . . . . . . . . . . . . . . . . . . . . . . . . 84

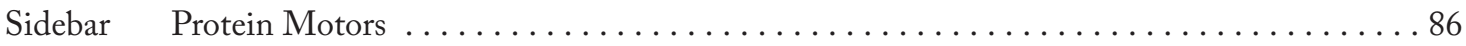

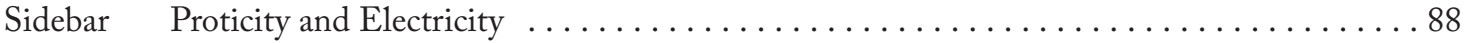




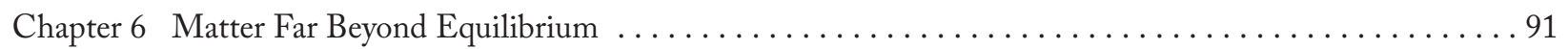

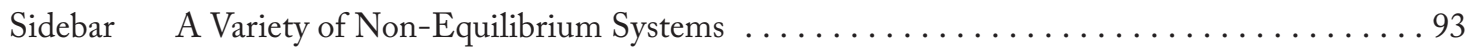

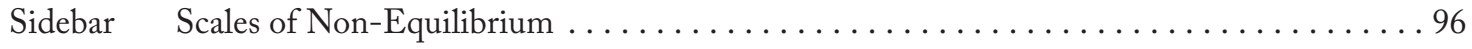

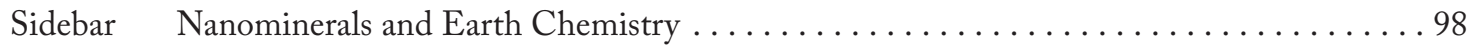

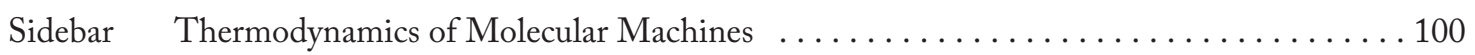

Sidebar $\quad$ Fluctuation Theorems .................................... 102

Chapter 7 Enabling Grand Challenge Science: The People and Tools Required . . . . . . . . . . . . . . . . 109

Sidebar Imaging Molecular Functionality $\ldots \ldots \ldots \ldots \ldots \ldots \ldots \ldots \ldots \ldots \ldots \ldots \ldots \ldots \ldots \ldots \ldots \ldots$

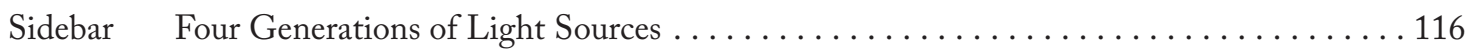

Appendix 1 The Grand Challenges Subcommittee Process .............................. 121

Appendix 2 Acknowledgements, Figure Sources, and Resource Materials . . . . . . . . . . . . . . 123 


\section{GRAND CHALLENGES FOR BASIC ENERGY SCIENCES}

It is frequently said that any sufficiently advanced technology is indistinguishable from magic. Modern science stands at the beginning of what might seem by today's standards to be an almost magical leap forward in our understanding and control of matter, energy, and information at the molecular and atomic levels.

Atoms - and the molecules they form through the sharing or exchanging of electrons - are the building blocks of the biological and non-biological materials that make up the world around us. In the $20^{\text {th }}$ century, scientists continually improved their ability to observe and understand the interactions among atoms and molecules that determine material properties and processes. Now, scientists are positioned to begin directing those interactions and controlling the outcomes on a molecule-by-molecule and atom-by-atom basis, or even at the level of electrons. Long the staple of science-fiction novels and films, the ability to direct and control matter at the quantum, atomic, and molecular levels creates enormous opportunities across a wide spectrum of critical technologies. This ability will help us meet some of humanity's greatest needs, including the need for abundant, clean, and cheap energy. However, generating, storing, and distributing adequate and sustainable energy to the nation and the world will require a sea change in our ability to control matter and energy.

One of the most spectacular technological advances in the $20^{\text {th }}$ century took place in the field of information, as computers and microchips became ubiquitous in our society. Vacuum tubes were replaced with transistors and, in accordance with Moore's Law (named for Intel co-founder Gordon Moore), the number of transistors on a microchip has doubled approximately every two years for the past two decades. However, if the time comes when integrated circuits can be fabricated at the molecular or nanoscale level, the limits of Moore's Law will be far surpassed. A supercomputer based on nanochips would comfortably fit in the palm of your hand and use less electricity than a cottage. All the information stored in the Library of Congress could be contained in a memory the size of a sugar cube. Ultimately, if computations can be carried out at the atomic or sub-nanoscale levels, today's most powerful microtechnology will seem as antiquated and slow as an abacus.

For the future, imagine a clean, cheap, and virtually unlimited supply of electrical power from solar-energy systems modeled on the photosynthetic processes utilized by green plants, and power lines that could transmit this electricity from the deserts of the Southwest to the Eastern Seaboard at nearly 100-percent efficiency. Imagine information and communications systems based on light rather than electrons that could predict when and where hurricanes make landfall, along with self-repairing materials that could survive those hurricanes. Imagine synthetic materials fully compatible and able to communicate with biological materials. This is speculative to be sure, but not so very far beyond the scope of possibilities.

Acquiring the ability to direct and control matter all the way down to molecular, atomic, and electronic levels will require fundamental new knowledge in several critical areas. This report was commissioned to define those knowledge areas and the opportunities that lie beyond. Five interconnected Grand Challenges that will pave the way to a science of control are identified in the regime of science roughly defined by the Basic Energy Science portfolio, and recommendations are presented for what must be done to meet them.

\section{FIVE GRAND CHALLENGES FOR BASIC ENERGY SCIENCES}

\section{- How do we control material processes at the level of electrons?}

Electrons are the negatively charged subatomic particles whose dynamics determine materials 
properties and direct chemical, electrical, magnetic, and physical processes. If we can learn to direct and control material processes at the level of electrons, where the strange laws of quantum mechanics rule, it should pave the way for artificial photosynthesis and other highly efficient energy technologies, and could revolutionize computer technologies.

- How do we design and perfect atom- and energy-efficient synthesis of revolutionary new forms of matter with tailored properties?

Humans, through trial and error experiments or through lucky accidents, have been able to make only a tiny fraction of all the materials that are theoretically possible. If we can learn to design and create new materials with tailored properties, it could lead to low-cost photovoltaics, self-repairing and self-regulating devices, integrated photonic (light-based) technologies, and nano-sized electronic and mechanical devices.

- How do remarkable properties of matter emerge from complex correlations of the atomic or electronic constituents and how can we control these properties?

Emergent phenomena, in which a complex outcome emerges from the correlated interactions of many simple constituents, can be widely seen in nature, as in the interactions of neurons in the human brain that result in the mind, the freezing of water, or the giant magneto-resistance behavior that powers disk drives. If we can learn the fundamental rules of correlations and emergence and then learn how to control them, we could produce, among many possibilities, an entirely new generation of materials that supersede present-day semiconductors and superconductors.

- How can we master energy and information on the nanoscale to create new technologies with capabilities rivaling those of living things?
Biology is nature's version of nanotechnology, though the capabilities of biological systems can exceed those of human technologies by a vast margin. If we can understand biological functions and harness nanotechnologies with capabilities as effective as those of biological systems, it should clear the way towards profound advances in a great many scientific fields, including energy and information technologies.

- How do we characterize and control matter away — especially very far away - from equilibrium?

All natural and most human-induced phenomena occur in systems that are away from the equilibrium in which the system would not change with time. If we can understand system effects that take place away-especially very far away—from equilibrium and learn to control them, it could yield dramatic new energy-capture and energystorage technologies, greatly improve our predictions for molecular-level electronics, and enable new mitigation strategies for environmental damage.

We now stand at the brink of a "Control Age" that could spark revolutionary changes in how we inhabit our planet, paving the way to a bright and sustainable future for us all. But answering the call of the five Grand Challenges for Basic Energy Science will require that we change our fundamental understanding of how nature works. This will necessitate a three-fold attack: new approaches to training and funding, development of instruments more precise and flexible than those used up to now for observational science, and creation of new theories and concepts beyond those we currently possess. The difficulties involved in this change of our understanding are huge, but the rewards for success should be extraordinary. If we succeed in meeting these five Grand Challenges, our ability to direct and control matter might one day be measured only by the limits of human imagination. 


\section{INTRODUCTION}

Civilization has been built upon the ability of humankind to exploit the material resources that nature has provided. From prehistoric tribes of hunters and gatherers who utilized wood-burning fires and fashioned tools from stone, to modern nations that run on machinery and processes primarily powered by energy from coal and oil, progress has been marked by increasingly advanced technologies designed to make use of our planet's bounty, either directly or by transforming natural materials into more useful substances.

However, as we move into the $21^{\text {st }}$ century, the finite and uneven distribution of natural resources is apparent, and many of our activities are adversely impacting the environment. Whereas it was once axiomatic that civilization would always advance into the future, questions of "sustainability" now arise. Here in the United States, it has also been taken for granted that such problems will be solved through science and technology. While science and technology can be the keys to a better tomorrow, there are significant intellectual challenges. These intellectual challenges are exacerbated by the current trend in which many of our best and brightest young minds are seeking careers in areas other than the physical sciences and engineering.

The basic ingredients of materials-biological and non-biological-are atoms, the smallest bits of matter that have the properties of chemical elements. Atoms can connect to one another to form molecules, the smallest bits of matter that retain the properties of chemical substances. Electrons, the glue that binds atoms into molecules, are negatively charged subatomic particles that can be shared or exchanged by neighboring atoms. It is the electrons, their dynamics and their properties, beginning with electrical charge and a property called "spin," that determine materials properties and direct chemical, electrical, and physical processes.

For all the vast and astonishingly diverse assortment of materials that we have learned to produce or fabricate, our discovery or invention of useful materials has historically resulted from a combination of trial-anderror experiments and lucky accidents. After some two and half million years, however, this situation is ripe for change. During the twentieth century, chemical synthesis and materials preparation began to be based more on control than on serendipity, resulting in advances ranging from new polymers to nanostructures. With the knowledge and tools (or capability for making the tools) that we currently possess, it is now within our grasp to achieve the ultimate in control of matter and energy. It is now realistic to think about one day being able to directly control molecules, atoms, and even the electrons that bind them. This ability will propel us into a "Control Age," where we design and create our own material resources, the resources that civilization needs.

Since the start of the $20^{\text {th }}$ century, it has become clear that the microscopic world in which atoms interact through the movement of electrons is not subject to the same classical laws of physics that govern the macroscopic world we encounter in our daily lives. The activities of electrons, atoms, and their molecular assemblies follow the counter-intuitive and often paradoxical rules of quantum mechanics, where, for example, a beam of light can function either as particles traveling in a straight line (photons) or as a wave propagating through empty space. During the twentieth century, scientists developed increasingly sophisticated technologies and instrumentation for the study of quantum effects. Our understanding of these phenomena has reached the point where we are ready to move beyond simple observation and take the steps that will enable us to direct and control matter and energy at the quantum level.

In the coming Control Age, scientists will be able to design and create entirely new materials and processes with desired properties and outcomes. With such capabilities, we should find solutions to some of the most vexing problems that civilization now faces, including energy, in all of its aspects, and changing global climate patterns. We should also find a great wealth of new science beyond problem-solving, including opportunities and possibilities we cannot even 
envision at this time. There is still much scientific groundwork that has to be done, however, before this Control Age can commence.

To define key needs in our scientific knowledge of matter and energy at the quantum level and to identify opportunities that await the acquisition of such knowledge, the U.S. Department of Energy's Office of Basic Energy Sciences (BES), one of the nation's largest sponsors of the natural sciences, convened a Grand Challenges subcommittee of the Basic Energy Sciences Advisory Committee (BESAC). This Grand Challenges subcommittee conducted a multi-faceted examination of the roadblocks to progress at the most fundamental level (see Appendix 1). This examination was based on a series of workshop reports, completed over the previous five years, on specific BES problems from catalysis to lighting. The results of that examination are presented in this report, titled Directing Matter and Energy: Five Challenges for Science and the Imagination.

The title reflects several aspects of this report. The twin aspects of energy and control (or direction) are the underlying concepts. Matter and energy are closely linked, and their understanding and control will have overwhelming importance for our civilization, our planet, our science, and our technology. This importance ranges even beyond the large portfolio of BES, both because these truly significant Grand Challenges confront many other realms of science and because even partial solutions to these challenges will enrich scientists' collective imagination and ability to solve problems with new ideas and new methods.

The Five Grand Challenges identified in the report roughly encompass the scientific areas defined by the BES portfolio. A truly Grand Challenge for basic energy sciences must be scientifically deep and demanding, be clear and well-defined, be relevant to the broad portfolio of BES, and promise real dividends in the form of devices or methods that can significantly improve the economy and quality of life for the United States. These five Grand Challenges were selected using those criteria. Over the past year, as the report was being assembled, the community provided a great deal of informal feedback, all of which was useful, and resulted in some honing of challenge names and emphasis. We believe that these five choices are the most appropriate and challenging topic areas for BES.

There are several deep scientific themes that link the Challenges together; Figure 1.1 illustrates this in an informal way. There is some overlap among Chapters 2 through 6 because these themes pervade all of them. These themes are the scientific issues that this report addresses. They are at the forefront of science today, and they will dominate and inspire the research agenda for basic energy science for the foreseeable future.

The next five chapters of this report describe the five Grand Challenges. Each chapter starts with an introduction that discusses the nature of the challenge and its importance both for fundamental understanding and for real-world applications. After that introduction, each chapter becomes more technical. These discussions provide details of each challenge and illustrate both the pure science and its possible applications.

Chapter 7 suggests some ways in which the Department of Energy can lead the scientific community in solving these Grand Challenge problems. Avenues discussed include developing the energy scientists of the $21^{\text {st }}$ century, developing the fundamental theoretical foundations for Grand Challenge science, and providing the advanced instruments and tools needed to go beyond observation towards control.

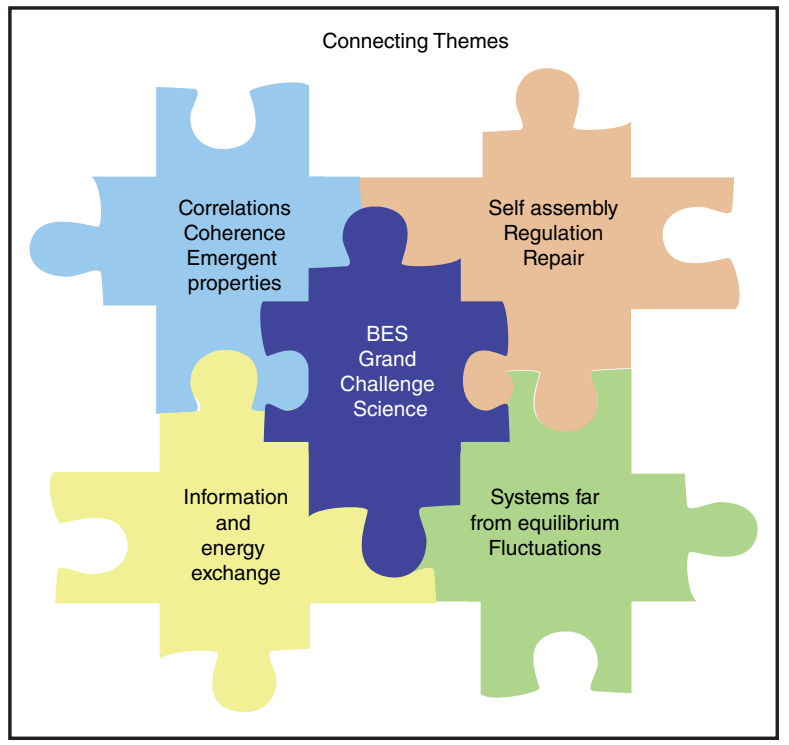

FIGURE 1.1. The major scientific themes underlying and connecting the five Grand Challenges. 


\section{FIVE GRAND CHALLENGES FOR BASIC ENERGY SCIENCES}

In the following, we identify the Grand Challenges, first describing each with a question and then following with a brief summary.

\section{Grand Challenge: How Do We Control Materials Processes at the Level of Electrons?}

In the coming decades basic energy science will shift from the study of how quantum systems are organized and assembled to the study of how they work and, ultimately, how to make such systems work for us. To date, the equations behind quantum mechanics have been far too difficult to solve, and the experimental methods to probe strong quantum correlations have been too crude. We will have to find ways to overcome these limits and improve our understanding of such quantum concepts as coherence and coherent interactions between matter and energy.

Coherence in quantum mechanics is a measure of the extent to which a wave field vibrates in unison with itself at neighboring points, enabling it to produce interference effects. Although quantum coherence is utterly absent from classical physics and only becomes evident on the microscopic scale of atoms and electrons, it can dominate the macroscopic properties of materials. For example, quantum coherence is responsible for superconductivity, the resistance-free flow of electrical current. Control of quantum coherence should lead to highly desirable material properties and processes in the $21^{\text {st }}$ century.

Elements of quantum control are exhibited by nature in various physical processes, including the harvesting of light during photosynthesis. Research in catalysis, photochemistry, molecular biology, and device physics could build on nature's techniques to achieve quantum-level control in human technologies. How can we produce energy in ways that are renewable and environmentally benign and that do not adversely impact global climate patterns? How can we detect harmful pathogens early, when their concentrations are small and more easily dealt with? How can we make

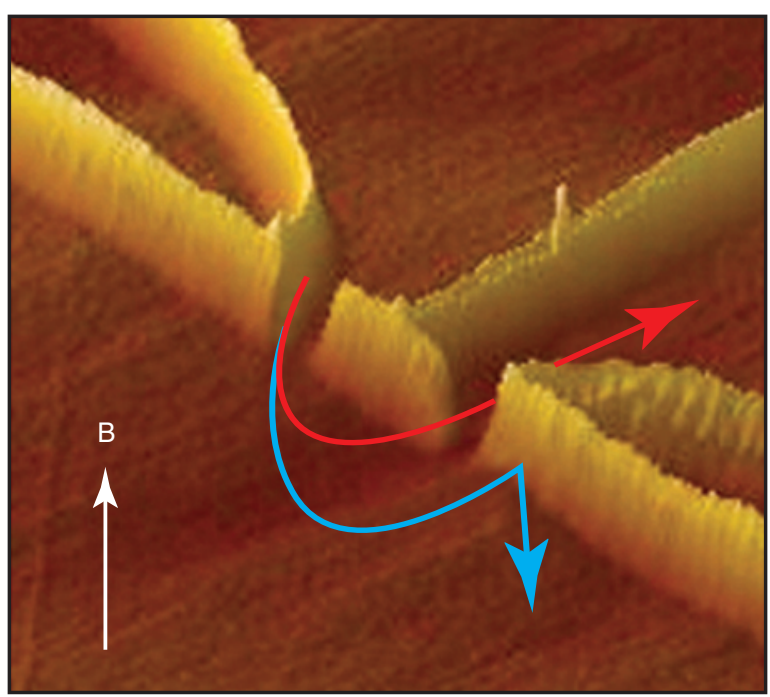

FIGURE 1.2. Atomic force micrograph of a device used to separate electrons according to their spin, a feature that could be used in next-generation "spintronics."

computers smaller, faster and better, and what are the true physical limits to doing this (Figure 1.2)?

\section{Grand Challenge: How Do We Design and Perfect Atom- and Energy-Efficient Syntheses of Revolutionary New Forms of Matter with \\ Tailored Properties?}

The terms "Stone Age," "Bronze Age," and "Iron Age" denote our increasing mastery over those materials. In the "Control Age," we will direct the movement of electrons in the "un-gluing" and "re-gluing" of atoms in chemical reactions and processes. This will enable the creation of new materials with properties tailored to meet the changing needs of civilization.

The creation of new materials and processes has always progressed hand-in-hand with advances in our ability to define the arrangements and transformations of atoms and electrons in matter and in our theories to explain and predict such phenomena. That only a tiny fraction of all possible chemical compounds has been prepared and their properties characterized points to great discoveries and technological pay-offs from further advances in our knowledge, if we can efficiently find or make the ones we want. 
Science is now tantalizingly close to the "directed" synthesis, guided by predictive design, of materials with tailored properties. How mechanically strong can we make materials yet keep them light-weight? How durable can our materials be? How resistant to corrosion, decay, or failure in extreme conditions of temperature, radiation, or environmental exposure? How electrically and thermally conductive? How transparent? How small yet functional? How biocompatible? Can we learn, like nature, how to make substances that repair themselves? Can we make materials that constantly modify themselves in response to changing environments?

These questions cannot be answered now, but they set the stage for the Grand Challenge of designing new materials with tailored properties to meet human needs (Figure 1.3). Over the next few decades, advances in theoretical understanding and computational power and methodologies could turn the design rules for materials upside down. For example, in a future world in which "reverse design" of materials is possible, scientists will start with a set of desired properties. They will then use theoretical models and computation to predict the specific type of atoms and atomic arrangement needed to obtain these properties and to create a recipe for producing the material. In this future world, materials designers should be able to produce truly extraordinary materials with properties we can now only guess at.

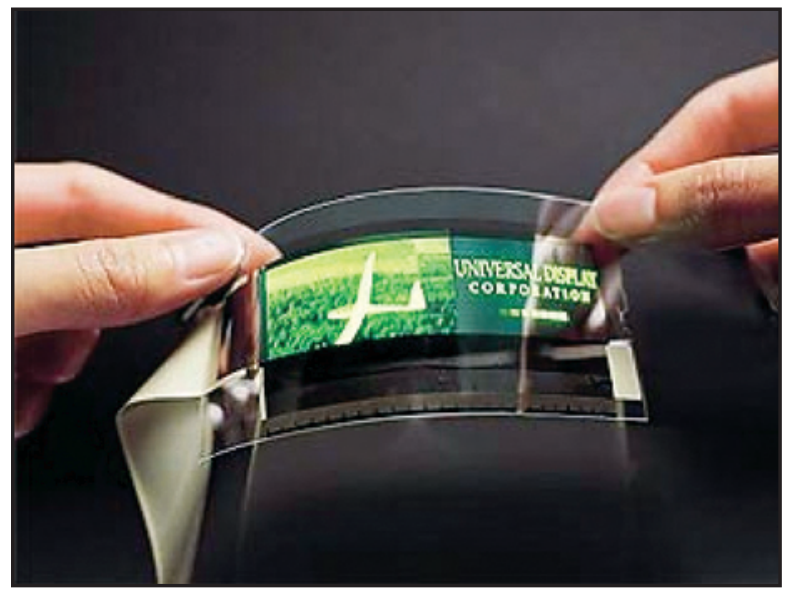

FIGURE 1.3. An OLED (organic light-emitting diode) utilizes flexible organic materials for hole and electron transport and recombination to achieve efficient light emission. Applications include more energy-efficient displays and solid-state lighting.
Grand Challenge: How Do Remarkable Properties of Matter Emerge from the Complex Correlations of Atomic or Electronic Constituents and How Can We Control These Properties?

"Emergent" phenomena, in which the correlated behavior of many particles leads to an unexpected collective outcome, are of great significance across a broad swath of science and engineering. In basic energy sciences, the particles may be microscopic like atoms, molecules, or electrons, or they may be larger entities such as sand grains, cells in an organism, or magnetic rocks in the earth's crust. One can readily find emergence in a multitude of phenomena, including crystalline materials (Figure 1.4), superconductivity, phase transitions, plasmas, climate change, self-assembly, cell colonies, and life itself. The human brain stands as one of the most stunning displays of emergent properties. The brain contains about 100 billion neurons, cells that transmit and receive electrochemical signals. Each neuron exists as an individual and rather simple cell, but from billions of them acting collectively there emerges the human mind.

Uncovering the fundamental rules of correlations and emergence is the first part of this Grand Challenge. The second part is to achieve control over those correlations, a prospect that can be contemplated because we now have the tools to probe and affect particles and their correlations on the nanoscale. Understanding and controlling correlations should enable us to put emergence to work for our benefit. The
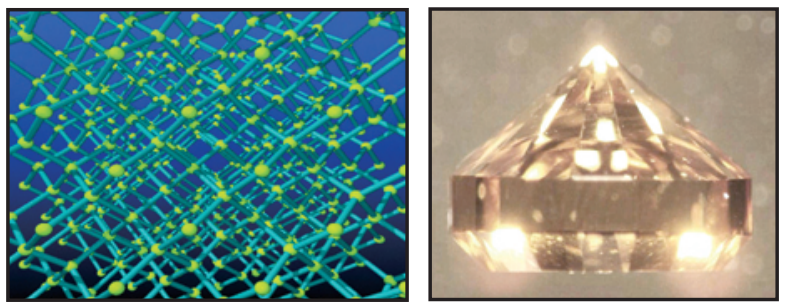

FIGURE 1.4. Left: The diamond lattice. Each carbon atom (yellow sphere) is bonded to four neighbors in a tetrahedral arrangement. The correlations in the atomic positions in the lattice persist over macroscopic length scales. Right: An artificial gem-quality diamond made by deposition of carbon vapor. Many of the extraordinary macroscopic properties of this diamond, such as its clarity and hardness, result from the perfect correlations of the atoms in the lattice. 
potential applications are as rich and diverse as the variety of emergent phenomena, but it is the collective properties of electrons in solids that are of special importance to basic energy sciences.

For example, today's information technology is based on semiconductors that, under normal conditions, are "weakly correlated" materials. This means that the motion of one electron is only influenced by other electrons in a relatively simple averaged way. While such simplicity has been a great benefit for modeling the function of semiconductors under ordinary conditions, it also has also limited that functionality. "Strongly correlated" materials, on the other hand, display collective electronic phenomena even under ordinary conditions. Such phenomena include magnetism, superconductivity and ferroelectricity.

If the precise processing and control that is routinely achieved for semiconductors today could be achieved for strongly correlated materials, it would open up remarkable technological possibilities. The Grand Challenge of understanding and controlling collective phenomena and emergent properties will require the extension of existing theories to new regimes, the development of fundamentally new theories, and the building of new instruments to control directly the atomic-level dynamics.

\section{Grand Challenge: How Can We Master Energy and Information on the Nanoscale to Create New Technologies with Capabilities Rivaling Those of Living Things?}

Biological systems are built of the same fundamental elements of matter and follow the same laws of physics as inorganic and human-engineered entities. Biological energy and chemical transduction, communication, adaptation, self-repair, and reproduction are all emergent properties. Look closely at biological nanomachines such as rotary molecular motors; they appear to resemble their human engineered counterparts. However, the way in which biological machinery works is often dramatically different from that of human-designed devices. In most cases, biological mechanisms hinge on physical behaviors that exist only at the nanoscale. Learning how biosystems utilize energy and fluctuations in their function can lead directly to new human-engineered devices.

While the design and fabrication of devices on the nanometer scale using solid-state electronic materials is becoming possible, most biological systems currently exceed (by a great margin) human-engineered nanotechnologies in what they can do. Biological systems are the proof-of-concept for what can physically be achieved with nanotechnology. Consider, for example, the ease with which biological systems transform and store energy, or their abilities to perform self-repair and to adapt to changing external conditions. The ways in which energy, entropy, and information are manipulated within the nanosystems of life provide us with lessons on what humans must learn to do in order to develop similarly sophisticated technologies.

The implementation and utilization of complex nanotechnologies with capabilities approaching and eventually exceeding those found in biological systems is quite beyond our present reach. To bring this level of nanotechnology under human control, we must answer several questions. Can the long-standing approach of "top-down" engineering be extended to the molecular level? Can we construct functional interfaces between biological systems and synthetic soft-matter and solidstate technology that directs the biological processes to suit our purposes? Or will we need to pursue a "bottom-up" approach, in which we develop design and fabrication techniques that enable the construction of synthetic devices with the functionalities of living systems-or, more likely, a blend of these two approaches?

The Grand Challenge of harnessing nanoscale phenomena will entail interfacing biological and nonbiological systems, which in turn will require communication, i.e., that we learn the chemical language of cells in order to convey information to them and ultimately domesticate them. To interface with living systems, we must also be able to handle their hardware configurations and connect nanoscale functions with the macroscopic world (Figure 1.5). Energy and information will also need to be controlled on the nanoscale. Issues must also be addressed pertaining to the fabrication and implementation of nanoscale devices. The size of nanoscale objects makes it feasible to utilize very large numbers of them with the possibility of radically new 


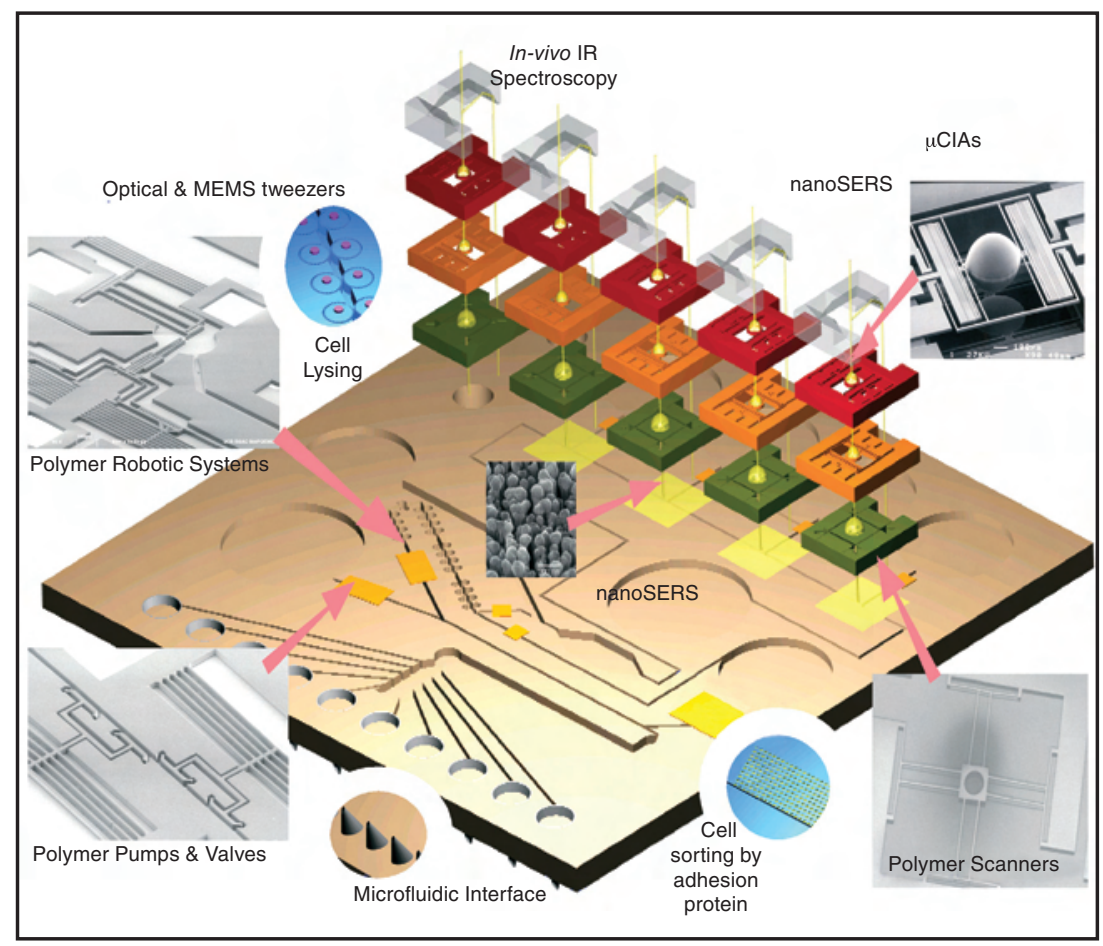

FIGURE 1.5. "Laboratory on a chip" includes an array of fluidic channels and ports for movement of chemicals. Optical and electronic detection systems, including nanoSERS (nano surface-enhanced Raman spectroscopy) and $\mu \mathrm{ClAs}$ (micro confocal imaging arrays), may be integrated as well into a truly hybrid device, combining elements from both biological and non-biological sides of the nanoscience revolution.

emergent properties. Meeting this Grand Challenge also paves the way for artificial, self-regulating, adaptive interactive devices.

\section{Grand Challenge: How Do We Characterize and Control Matter Away-Especially Very Far Away-from Equilibrium?}

A system is in equilibrium when it does not change with time. All natural and most human-caused phenomena occur away from equilibrium. Away-fromequilibrium systems can range in scale from the very small (nanostructures and bacteria) to the very large (earthquakes), and away-from-equilibrium processes occur on timescales ranging from nanoseconds to millennia. Despite the pervasiveness of these nonequilibrium systems and processes, most of our current understanding of physical and biological systems is based on equilibrium concepts.

It has long been recognized that understanding matter and information systems away from equilibrium is a Grand Challenge to science and engineering. Understanding non-equilibrium behavior of physical, chemical, biological, atmospheric, geological, and even astronomical phenomena promises huge advances in our ability to manufacture super-hard, super-strong, and self-repairing materials, and in a great many other areas as well, from earthquake prediction to the control of complex structures and even phase stability. Understanding non-equilibrium phenomena will also help us optimize processes for obtaining, transducing, and storing energy from sources such as wind, geothermal, nuclear, and fossil fuels through advanced batteries and fuel cells.

Meeting the Grand Challenge of non-equilibrium phenomena will require that we answer a number of complex questions. What are the general rules that apply to microscopic relaxation time scales that are very long? Can we control structural geometry or direct processes at the nanoscale? Can we deal with nonequilibrium and fluctuations? Can we understand enough about biological repair mechanisms to use them (or related schemes) in molecular and solid-state device applications? Can we comprehend how systems search free-energy landscapes? Can efficiency measures be developed for cellular and artificial cellular processes and for highly non-equilibrium processes? How do we extend these considerations to deal with power, and how do we design machines that operate far from equilibrium to produce power and to do so 
efficiently? Can we understand non-equilibrium processes in biology well enough to mimic them with artificial systems (Figure 1.6)? Can we use multipletemperature phenomena for energy control? Can we stabilize matter in non-equilibrium states, such as metamaterials, high-strength glass or graded structures? Can we understand and control turbulent flows that pertain to phase transitions, diffusion, and chemical reactions?

Because of the pervasiveness of non-equilibrium phenomena, mastering this Grand Challenge should pave the way for effectively dealing with a host of issues facing civilization, including energy, climate, materials, biology, and even security.

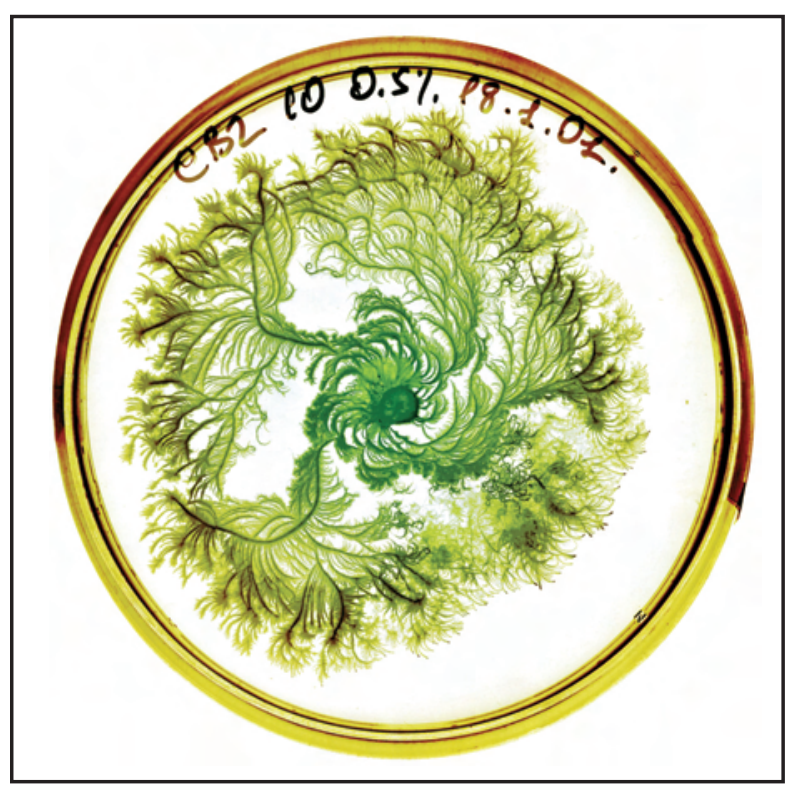

RECOMMENDATIONS: WHAT WILL WE NEED TO MEET THE FIVE GRAND CHALLENGES FOR BASIC ENERGY SCIENCES?

Making the leap from observation science to control science is the collective goal of the Five Grand Challenges for Basic Energy Science and will provide the United States with the deep expertise needed to address the overarching national priority of a secure energy future. It requires a three-fold attack: new approaches to training and funding, creation of theories and concepts beyond those we currently possess, and development of instruments that are more precise and more flexible than those used for observation science.

The transition from observation science to control science envisaged in the five Grand Challenges depends upon finding the right people, including a young and diverse set of scientists (Figure 1.7), and supplying them with the right tools. Providing this mix will require new ways and methods of addressing the training and support of scientists, and the development of new instrumentation. Such efforts will have to be sustained and carried out across multiple areas of research. In addition, because meeting the five Grand Challenges promises to radically reshape many of the technologies that are critical to solving some of the world's most vexing problems, including nearly all aspects of energy, Grand Challenge scientists will also need to have a
FIGURE 1.6. One of several striking patterns that bacteria, in this case tens of billions of them, form when grown in petri dishes. The colony structures form as adaptive responses to laboratory-imposed stresses that mimic non-equilibrium hostile environments faced in nature.
FIGURE 1.7. To attack the Grand Challenges and to provide the United States with the deep expertise needed to address the overarching national priority of a secure energy future, it is critical to have the right people, including a young and diverse set of scientists.

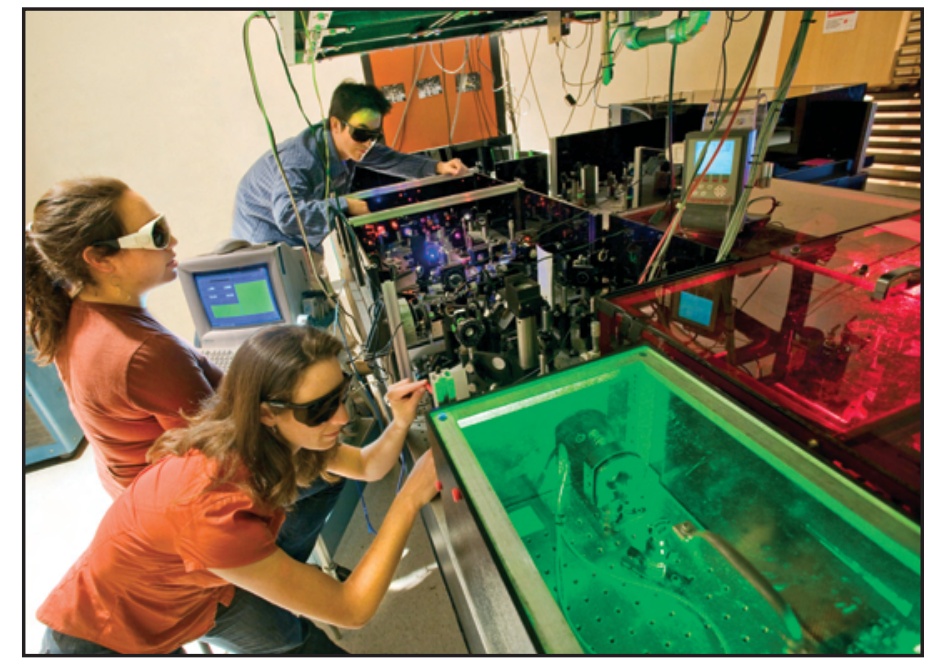


heightened awareness of the social, diversity, industrial, and technological contexts of their research. We will need to foster, nuture, and reward excellence and creativity in research and in all aspects of education.

To effectively attack the Five Grand Challenges, science will also require theories and concepts beyond those we currently possess and instruments that are more precise and flexible than those we now use. Directing and controlling the properties of materials for a desired outcome requires the ability to see functionality at the length, time, and energy scales at which it takes place (Figure 1.8). We will need to interrogate matter at a level much deeper than the macroscopic averages we study today, so that the properties of individual molecules or atoms of a material can be observed and controlled. We therefore need to develop and disseminate new tools capable of viewing such inner workings of matter as transport, reactivity, fields, excitations, and dynamics.

Richard Smalley won the Nobel Prize for his discovery of a new form of carbon. He argued that the problem of energy confronts the human race today

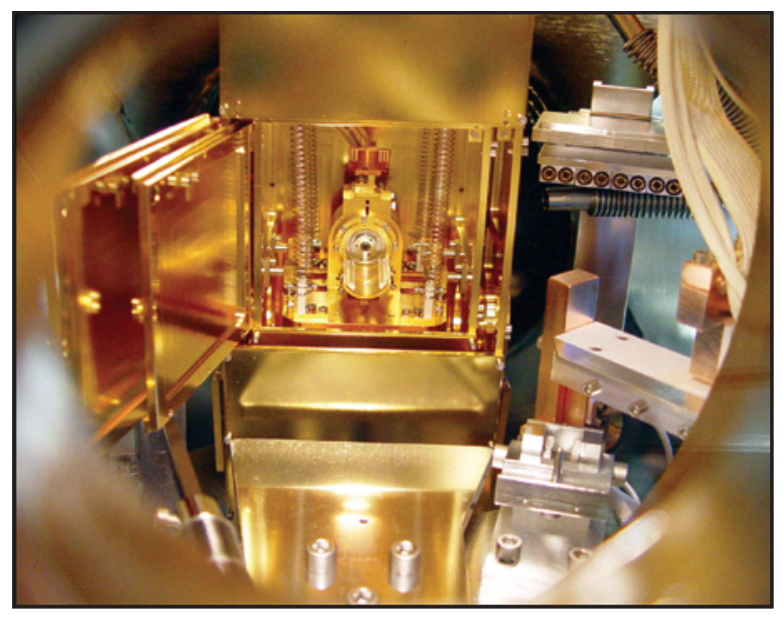

FIGURE 1.8. This cryogenic, variable-temperature ( $8 \mathrm{~K}$ to $300 \mathrm{~K})$, ultra-high-vacuum scanning tunneling microscope (UHV STM) optimized for single-molecule studies on semiconductors exemplifies the experimental capabilities needed for imaging functionality in Grand Challenge science.

with immediacy and danger. He felt that understanding and mastering energy science could have great and positive effects on our civilization, our economy, and our people. This report, written in that spirit, focuses on Five Grand Challenges for Science and the Imagination. 


\section{QUANTUM CONTROL OF ELECTRONS IN ATOMS, MOLECULES, AND MATERIALS: CREATING A NEW LANGUAGE FOR THE BEHAVIOR OF ELECTRONS}

The Grand Challenge for Quantum Control of Electrons is to control material processes at the level of electrons.

One of the most significant new paradigms in quantum research may build on applied quantum physics, whose goal is to facilitate the transition from science to engineering of processes involving quantum coherence. Basic quantum research in physics and chemistry laboratories may hold solutions to some of the world's most important technological problems: How can we produce enough energy? How can we detect harmful pathogens early, when their concentrations are small? How can we make computers smaller, faster, better, cooler, and what are the true physical limits to computation? How can we control the spread of new diseases or the increasing drug-resistance of old ones? Research in catalysis, photochemistry, molecular biology, and device physics may deemphasize discovery of nature's solutions in favor of quantum-level synthesis and control of new, human-devised solutions to these problems. We need to go beyond the study of how quantum systems are put together and organized to the study of how they work. And we need to go still further, beyond how they work to how to make quantum-scale systems work for us.

Much of the last century has focused on understanding how electrons in matter-their charge, their spin, and their dynamics - determine the properties of materials and how they direct chemical, electrical, or physical processes in materials. We are now on the verge of a new science of quantum control where our tools will go beyond probing what is there, towards the goal of controlling these processes and properties through direct manipulation of the electrons.
This chapter describes the challenges of understanding and controlling the electrons in matter. Much of the work at the frontier of this field concentrates on the concept of coherence and its relationship to control. Coherence in quantum mechanics refers to the effects of well-defined phase. It is a measure of the extent to which a wave field vibrates in unison with itself at neighboring points and so is capable of producing interference effects. In sound waves, this leads to beat notes. In light, coherence is responsible for the speckled appearance of a laser beam on a flat surface. In quantum mechanics, coherence may occur in the quantum phase, a property of matter that usually only becomes manifest on the tiny scale of atoms and electrons and is utterly absent from the classical description. Yet quantum-phase correlation can dominate the macroscopic properties of materials; for example, it is responsible for superconductivity, the resistance-free flow of electrical current in some cooled metals. Quantum correlations also produce giant magnetoresistance, which is used to read computer hard drives. Our mastery and control of quantum coherence may lead to other highly desirable material properties and processes in the $21^{\text {st }}$ century.

Chapter 4 in this report will describe some of the challenges in understanding phenomena that arise from quantum phase correlations. This chapter focuses on exploring the challenge of controlling coherence.

Control should not be viewed as achievable only by human design. Nature also implements quantum control in various physical processes, and we have much to learn from it. Ubiquitous yet mysterious phenomena such as light harvesting by photosynthesis may have as much to do with quantum control as any laboratory quantum information processor. In this chapter, we link these themes together. 
MATERIALS, THEIR COHERENCE-

BASED PROPERTIES, AND HOW WE MAY CONTROL THESE PROPERTIES IN THE FUTURE

\section{Motivation and Connections to Materials}

The $20^{\text {th }}$ century witnessed spectacular progress in our understanding of the inherent properties of isolated atoms, molecules, and their aggregates, and their reactions to external influences. The progress can be appreciated by recalling the opposition to the atomic hypothesis (that is, the notion that atoms exist) by the physicist Ernst Mach and chemist Wilhelm Ostwald at the start of that century.

The scientific challenges at the beginning of the $21^{\text {st }}$ century will, no doubt, include further significant clarifications of deep physical and chemical issues, such as the mechanism of high-temperature superconductivity and the physical implementations of powerful quantum computers. Several of these are discussed at greater length in other chapters of this report. In the present chapter, the main emphasis will be on the exciting, new field of coherent dynamical control of atoms, molecules, and their aggregates by lasers and other coherent perturbations, from weak to very strong (laser intensities of greater than $10^{20} \mathrm{~W} / \mathrm{cm}^{2}$ are now available) at the length and time scales of atoms and molecules themselves [angstroms and attoseconds $\left(10^{-18} \mathrm{~s}\right.$ or as)]. This challenge promises to open up entirely new areas of science with applications largely still unexpected. For example, will there be programmable quantum simulators that can model the behavior of nuclear matter? Will drugs be designed and assembled for testing by using coherent properties of lasers? Such visions may not be realized soon, but there are sure to be unanticipated applications that are just as important and far-reaching.

The collective behavior of electrons in solids is responsible for many remarkable properties and technological applications. This collective behavior has not yet been understood because of the great challenge of describing systems of strongly interacting electrons and ions. This topic is also discussed in Chapter 4.

\section{Superconductivity and Coherence}

Quantum coherence is not only seen in carefully controlled materials; it can occur in natural systems. One of the most remarkable phenomena in condensed matter science is the sudden, total disappearance of electrical resistance of many metals and compounds as the temperature is lowered through a material-specific critical temperature $\left(\mathrm{T}_{\mathrm{c}}\right)$. Discovered in 1911 in lead $(\mathrm{Pb})$, whose critical temperature is 7.2 degrees above absolute zero, it took over 40 years before it was explained by Bardeen, Cooper and Schrieffer. The phenomenon is governed by the so-called gap function, an inherent and coherent quantum mechanical property in a superconductor that is in some respects similar to the coherent field of laser light.

In 1986, a new family of superconductors, so-called high-temperature (or cuprate) superconductors, was discovered by Mueller and Bednorz. They exhibited much higher critical temperatures, but the essential coherence properties were of the same nature. Cuprate materials are composed of metallic copper-oxygen $(\mathrm{CuO})$ planes separated by insulating spacers. Yet from this modest origin emerge many distinct electronic behaviors, superconductivity being one of them. Understanding the underlying physics of high- $\mathrm{T}_{\mathrm{c}}$ superconductors requires novel experiments, innovative theory, and the development of new computational methods.

\section{Correlation and Control}

Control may be particularly important in materials that exhibit strong correlation, where the properties can be easily manipulated by externally applied forces. For example, the magnetic moments associated with the spins of individual electrons in a ferromagnet can qualitatively be regarded as coupled parallel, or ferromagnetically, to each other. Another way of thinking about this is that a given spin is acted on by an effective magnetic field due to the other spins that line it up parallel to a common direction. This effective field is phase-coherent and points in the same direction everywhere.

For these reasons, sophisticated magnetic materials can be controlled for information storage. There are 
opportunities to expand magnetic correlation and control in the future, to devices such as nanoscale sensors as well as whole technologies, which have come to be known as spintronics (Figure 2.1).

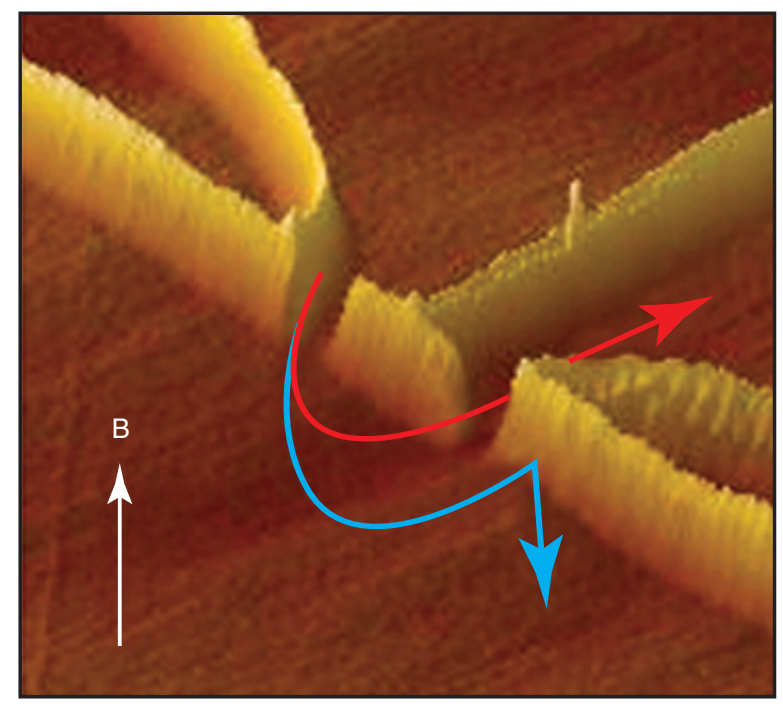

FIGURE 2.1. Atomic force micrograph of a device used to separate electrons according to their spin, a feature that could be used in next-generation "spintronics."

\section{COHERENT CONTROL OF EXCITED ELECTRONIC STATES}

New quantum-coherence examples in photochemistry and photocatalysis concern the control of excited electronic states in molecules and on surfaces. For example, the molecular dynamics of combustion is significantly affected by the presence of molecules in electronically excited states. Increasing the overall efficiency of new fuels that come from biomass and reducing pollution from new and existing fuels require that we understand these transitions in detail; however, even the simplest example is nearly beyond our grasp at present.

The root of the very challenging problem of controlling chemistry is the quantum-mechanical behavior of the electrons. Electrons, of course, are the glue of chemistry: chemical bonds are simply electrons that are shared between atoms. Reactions - the making and breaking of bonds-just come down to electron dynamics.
Electrons must be treated quantum mechanically when they form chemical bonds because they are simply too light to follow the rules of Newtonian mechanics in the confined space within a molecule. Quantum mechanics is more difficult than classical mechanics of discrete particles because it requires that the electrons be treated as waves.

The nuclei in chemical reactions are much heavier than electrons and so we can often treat them as slow-moving objects within a landscape determined by the fast-moving electrons; this is known as the BornOppenheimer approximation (BOA). But even so, the electron problem is still formidable.

\section{Challenge: The Transition State (TS) Concept in Chemical Reactions Does Not Always Work}

Consider, as a simple example, the reaction of a hydrogen atom $(\mathrm{H})$ with a formaldehyde molecule $\left(\mathrm{H}_{2} \mathrm{CO}\right)$. The forces on the atoms depend on the position of their shared electrons, which in turn depend on nine distances and angles between the atoms in the reaction. As the atoms move, the electrons rearrange, and the forces change. Chemical physicists have adopted the notion of a potential-energy landscape or surface, a kind of imagined curved sheet, where atoms can roll around like peas on a warped dinner plate (see Figure 6.3 in Chapter 6).

Calculating such surfaces has occupied much effort in the $20^{\text {th }}$ century and resulted in very sophisticated modeling programs that do a pretty good job. We can calculate that stable reactants and products are $\mathrm{H}+\mathrm{H}_{2} \mathrm{CO}$ and $\mathrm{H}+\mathrm{H}+\mathrm{HCO}$, or $\mathrm{CH}_{3} \mathrm{O}$, and we can also see that the atomic pathways that lead from one of these stable configurations to another go over high places (saddle points) on the surface that require some kinetic energy to reach. There are successful approximate models called transition-state theories that use simple statistical ideas-basically considering the relative size of the transition saddle point to the total space of dynamics on the surface-to calculate the rate of these reactions as temperature and pressure are varied. All this knowledge represents a triumph of computational sophistication. However, this is just a tiny corner of the real problem. 
It is known that transition-state theory cannot predict some reactions because evidently they do not take the simple path over the mountain pass in the potential energy surface. For example, one of the important final states in the $\mathrm{H}+\mathrm{H}_{2} \mathrm{CO}$ reaction is the formation of the molecular hydrogen in the products $\mathrm{H}_{2}+\mathrm{HCO}$. For these situations, larger calculations are required that may involve the interaction of solvent molecules, and these are much more complicated. Full quantum calculations are quite impossible because of their tremendous size and complexity. Basically, a quantum calculation of the motion of atomic nuclei on a single Born-Oppenheimer surface scales not as the number of atoms, but as a power of the number of atoms. To be specific, the amount of effort required to calculate the interatomic motion of $\mathrm{n}$ interacting atoms scales as the $3 \mathrm{n}$ power! (see Sidebar "Theoretical Challenges of Quantum Calculations"). We quickly run into a problem identified by Walter Kohn as the "exponential wall" of difficulty in calculating using the rules of quantum mechanics.

Even this complication, challenging as it is, only represents a tiny step towards the whole solution.

\section{A Bigger Challenge: Excited States}

The real trouble starts when we consider the effects of radiation and excited states of electrons; that is, not just kinetic energy, but photoexcitation. Problems like combustion or light harvesting by photosynthesis are important examples. Light excites electrons to higher states, which can - and in fact usually do-have completely different potential-energy surfaces. The photochemistry is therefore completely different when we take into account the transfer of energy among excited electronic states. The computational tools developed for ground-state molecular structure and dynamics are of limited utility here.

An example of this problem that has been studied extensively is bacteriorhodopsin (bR), a protein that absorbs light and stores its energy by transporting protons across a membrane. Bacteriorhodopsin is closely related to rhodopsin, the primary light detector in vision. In the center of the protein is a light-absorbing molecule called retinal (Figure 2.2). It first collects a photon and then undergoes a sub-picosecond twist around a double bond, which triggers a chain of events that pumps a

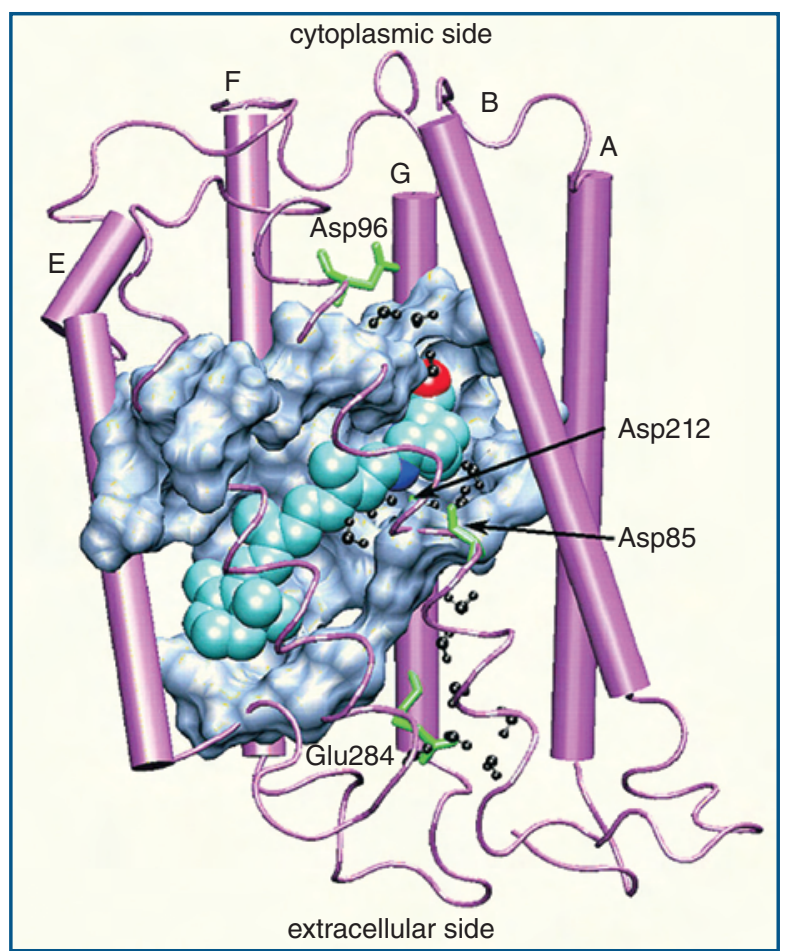

FIGURE 2.2. The retinal molecule (light blue) in the center of rhodopsin initiates a complex series of events that moves protons as part of the process of vision.

proton across the membrane. This process involves multiple electronic states, so it cannot simply be described as pushing atoms around on a potential-energy surface governed by Newtonian mechanics.

\section{Quantum Coherence: a Still Bigger Challenge}

The calculation of dynamics on two or more different potential-energy landscapes (governed by the absorption or emission of photons by the binding electrons) is a daunting problem to confound the fastest computer, but the real physics is even deeper than that. A further challenge, which makes the other look like a simple child's game, involves the issue of quantum coherence in molecular dynamics, i.e., the simultaneous evolution of a system on multiple potential-energy surfaces, and issues like entanglement.

Schroedinger's cat, quantum teleportation, quantum computation, the Einstein-Podolsky-Rosen (EPR) paradox, spooky action at a distance- these are all popular catch phrases that refer to the fact that the rules of quantum mechanics cannot be understood simply from the classical analogs of waves sloshing in a bathtub. So 


\section{THEORETICAL CHALLENGES OF QUANTUM CALCULATIONS}

As experimental methods become rapidly more capable of controlling the statics and dynamics of many-atom quantum systems (nanostructures, proteins, Coulomb explosions, attosecond physics), there is a corresponding theoretical challenge (Figure 1). The underlying problem is that the complexity of $\mathrm{N}$-atom wave functions grows exponentially as a function of $\mathrm{N}$, and currently becomes unmanageable when $\mathrm{N}>\overline{\mathrm{N}}$ where $\overline{\mathrm{N}}$ is in the range 10-50. Density functional theory extends this range for static problems to 100-1000. Great challenges for the next decades are to develop new methodologies to increase $\overline{\mathrm{N}}$ for static systems into the range of 1000-10,000. For dynamic

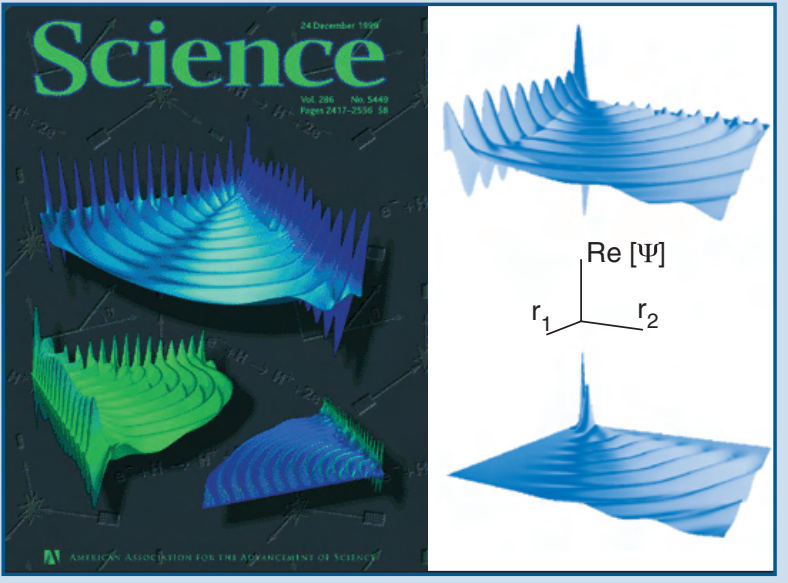

FIGURE 1. Left: Visualization on the cover of Science of three of the hundreds of possible components of the quantum wave function for a two-electron system broken by collision with an electron. Right: Two of the many components of the quantum wave function for two electrons being ejected from the $\mathrm{H}_{2}$ molecule by a single photon. systems, reasonable targets are $\overline{\mathrm{N}}=10-100$ for low excitation energies $(\Delta \mathrm{E}<1-10 \mathrm{eV} /$ atom $) . \overline{\mathrm{N}} \approx 10$ for higher energies. Such theoretical progress would have a major impact on structural and energetic aspects of the chemistry of large molecules, on material science, and on the understanding and control of chemical reactions.

An even greater conceptual challenge is learning to deal with the increasingly studied regimes in which nuclear and electronic dynamics cannot be separated, i.e., in which the Born-Oppenheimer approximation is not applicable. it should not surprise us that the practical worlds of photochemistry and biophysics are not immune from the laws of quantum coherence. These rules present computational difficulties that are currently intractable. But they also present opportunities to solve big problems in new ways.

\section{Connections to Nature: Photosynthesis}

Plants and bacteria harvest light for photosynthesis in ways that we do not yet understand in detail; however, the earth, both land and sea, is covered with the solution-the sun! The energy reaching the earth from our sun is a staggering hundred-thousand-trillion $\left(10^{17}\right)$ watts. That's more than human civilization needs by a lot! The typical house in a suburb or town in America has far more solar power hitting its roof, and vastly more solar power hitting the property it rests on, than the family inside consumes for electricity and heat, even with our American wastefulness. Some U.S. households are beginning to use this power. The solar power source is abundant, but the challenge is to find cheap and efficient ways to harvest the power.

Very recently there were clues that some kinds of photosynthesis may utilize the same quantum coherent behavior used for qubits, the internal working parts of a quantum computer (see Sidebar "Entanglement"). In this case, evolution may have built qubits into the mechanism for photosynthesis. What researchers have found is that the transfer of energy from light to chemical potential energy during photosynthesis (in a particular system found in the green sulfur bacterium) is absolutely dependent on electronic coherence (Figure 2.3). Hence, the electron that is excited by absorbing a photon does not just move through the 


\section{ENTANGLEMENT}

The power of quantum computing comes from a peculiar feature of quantum mechanics called entanglement, which can be illustrated easily using only two particles or degrees of freedom, a and b, which carry information through their quantum state. Often one uses spins to represent this, and in the simplest case, each particle can be in one of only two spin states ("up" represented by $|\uparrow\rangle$ ) or ("down" represented by $|\downarrow\rangle)$. These particles are called quantum states, or qubits, and because they are quantum mechanical, they can be in a superposition of both of their spin states at once. Among the possible states of the system, represented by $|\Psi\rangle$, where both qubits are up and down simultaneously are these two:

$$
\begin{aligned}
& \left|\Psi_{\text {sep }}\right\rangle=\left[\left|\uparrow_{a}\right\rangle+\left|\downarrow_{a}\right\rangle\right]\left[\left|\uparrow_{b}\right\rangle+\left|\downarrow_{b}\right\rangle\right] \text { and } \\
& \left|\Psi_{\text {ent }}\right\rangle=\left|\uparrow_{a}\right\rangle\left|\downarrow_{b}\right\rangle+\left|\downarrow_{a}\right\rangle\left|\uparrow_{b}\right\rangle .
\end{aligned}
$$

The first of these states is said to be "separable" because measurement of either qubit will yield either up or down, separately. The result of each measurement will be independent of the measurement of the other.

The second state is a lot weirder because the measurements do not separate. If we measure the state of atom a, it may be up or down, and the result is random. But if we measure a to be up, then for this state $\mathrm{b}$ is down with certainty. Conversely, if $\mathrm{a}$ is down, $\mathrm{b}$ will definitely be found to be up. Thus while the fate of each atom is random, these fates are inextricably linked or correlated. This is what we mean by entanglement, visually represented by the "ambiguous cube" in Figure 1.

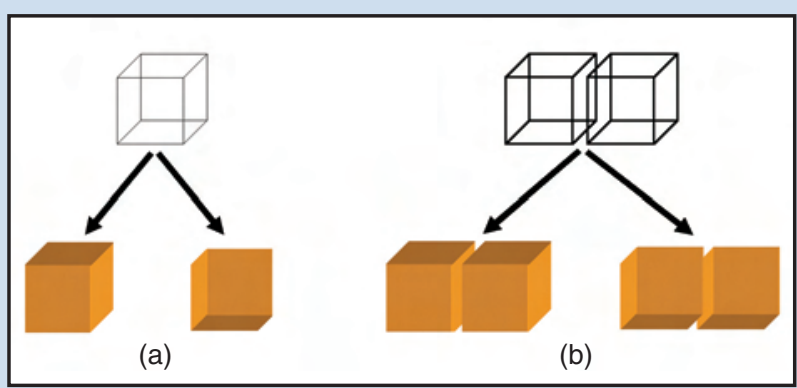

FIGURE 1. The "ambiguous cube" visually represents superposition and entanglement. Left: The mesh cube represents a superposition of a single qubit in orientations tilting up and down. A measurement is represented by your ability to mentally lock it into one or the other orientation, as shown in the lower shaded cubes. Right: This situation is analogous to an entangled superposition of two qubits, where measurement of either cube locks in your perspective on the other one, even though they have no physical connection.

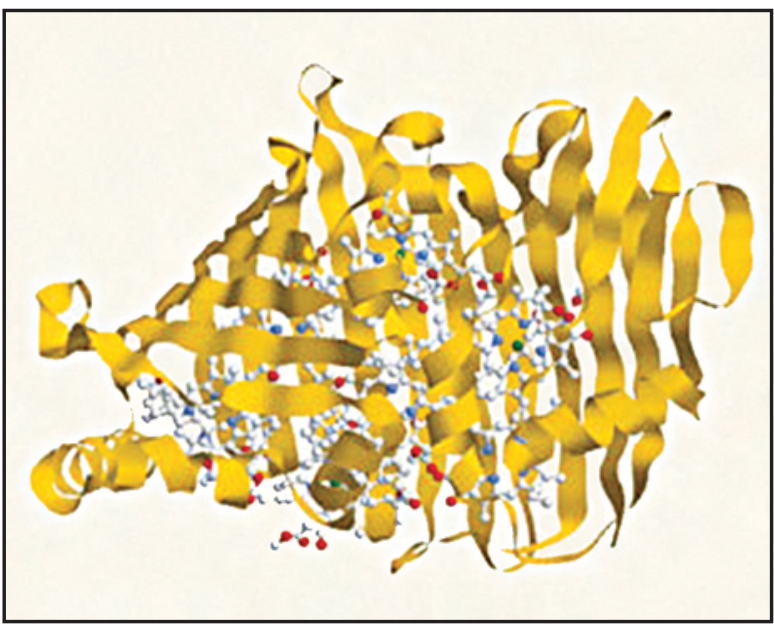

FIGURE 2.3. A structural cartoon, derived from protein crystallography, of the Fenna-Matthews-Olsen antenna complex that appears to utilize quantum coherence in the process of photosynthesis. Yellow: protein; white: chromophores. chromophore like a classical particle, which moves under the influence of local fields in the molecule, but rather has to transit simultaneously through more than one energy state in the system. This "two states at one time" concept is standard fare for quantum mechanics, but it is absolutely impossible to describe this classically, so much so that our language to describe it is inadequate. Hence we say the electron is in a superposition of different states - two states at one time.

This recent discovery was surprising because quantum coherence is known to be very fragile, and the messy, complicated environment of a bacterium at room temperature was thought to mean death to anything so delicate as electronic superposition. The molecule appears to have been constructed to protect itself from decoherence. This is yet another important lesson 
that we stand to learn from studies of light-harvesting systems.

\section{Going Beyond the Born-Oppenheimer Approximation}

The Born-Oppenheimer Approximation discussed above is a cornerstone of computational chemistry. The separation of time scales between the light electrons and heavier nuclei does more than just simplify the calculation of molecular properties and dynamics. It also provides a conceptual basis for understanding the physics by providing a way to visualize what is happening during a reaction.

Perhaps it is the strong sense of reality that comes from visualizing dynamics on potential-energy surfaces that makes the BOA so universal. However, it has been clear for some time that there are many situations where the approximation breaks down because the nuclear and electronic dynamics are coupled-and the results may be unexpected, or at least not obvious from dynamics on the potential-energy landscape.

A very important class of situations where the BOA breaks down is non-radiative decay of excited polyatomic molecules. When there are more than two atoms in a molecule, there are usually independent intramolecular degrees of freedom that must be described by a higher-dimensional potential-energy landscape. For example, for a tri-atomic molecule, there are two different bond-stretching modes plus one bending mode. In this case there are almost always points in the landscape (that is, specific geometrical arrangements of the atoms), where two different quantum levels of the electrons have the same energy, i.e., are degenerate. This energy degeneracy spoils the BOA because, in the vicinity of the degeneracy, the electronic and nuclear motion are strongly coupled. These degeneracies are called "conical intersections" because in a two-dimensional projection of the potential-energy landscapes, they look like points where two surfaces touch, like the apex of a pair of cones (Figure 2.4). Conical intersections are points where electron wave-functions in molecules can undergo large changes, often on the timescale of a few femtoseconds (fs), during chemical reactions.

Obviously, these are regions of intense interest for physical chemistry, and understanding the behavior of

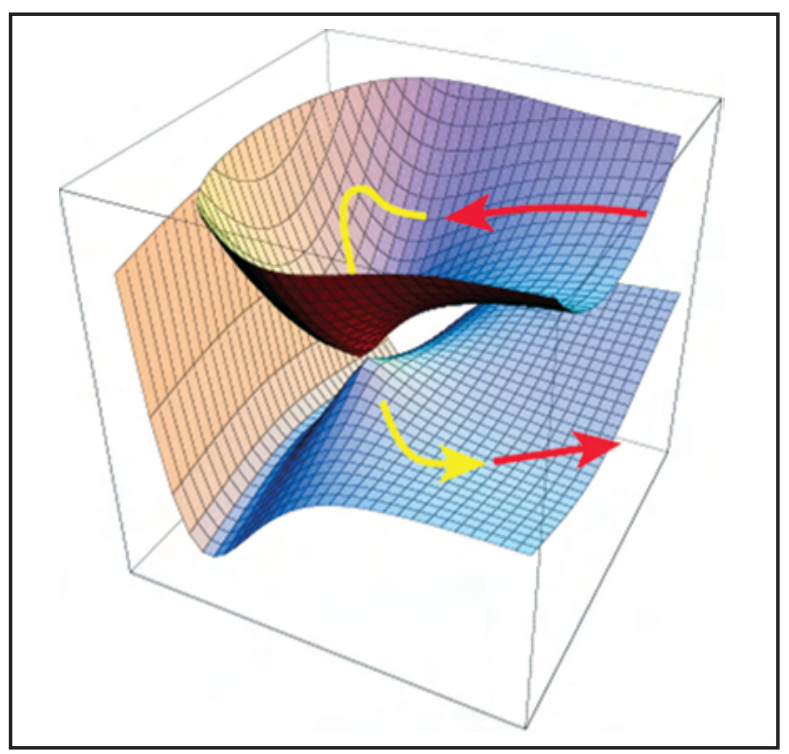

FIGURE 2.4. Conical intersection, the point of contact between the potential-energy surfaces of two different electronic states in a molecule.

molecules as they pass through conical intersections may point us in the direction of a new language of electron dynamics in material systems (see Sidebar "Conical Intersections and Wavepacket Dynamics").

\section{COHERENCE AND CONTROL IN MATTER AT HIGH OR LOW TEMPERATURES: COLD, COOL, WARM, AND HOT MATTER}

\section{Our Everyday World Is Cool}

"Ordinary" matter at room temperature- the matter we rely on to build everything from rocket ships to paper clips-is actually quite cool, in the sense that the thermal energy $\mathrm{kT}$ is far lower than the kinetic energy of the electrons responsible for the familiar shiny, malleable, and conducting properties of metals. The high electron energies are a consequence of quantum mechanics, which forces electrons to have a higher energy (their "Fermi temperature") when they are packed into solids. Likewise, the Coulomb binding energy that holds atoms together is much greater than $\mathrm{kT}$, which is why solid material exists. Much of the vast research in condensed-matter physics and materials engineering is devoted to understanding the properties of cool matter. 


\section{CONICAL INTERSECTIONS AND WAVEPACKET DYNAMICS}

In the conventional picture upon which chemists base their understanding of how most chemical reactions occur, each electronic state of a molecule is characterized by a multidimensional potential-energy surface upon which the nuclei move as the molecule vibrates or dissociates. This is the simple Born-Oppenheimer picture. When there are more than two atoms in a molecule, these surfaces can touch in a geometry that, when viewed in terms of two vibrational degrees of freedom, is equivalent to the contact of two cones at their apexes, as shown in Figure 2.4. If a molecule is prepared in an excited state represented by the upper surface, it can quickly evolve to a state on the lower surface through the common point (or set of points) that joins them, as shown by the arrows in that figure. The quantum description of that process can be seen by following the fate of a quantum wave packet, describing the motion of the nuclei, prepared on the upper surface, for example by the absorption of a photon. The two surfaces involved in such a process are depicted separately in the top row of Figure 1. A calculation based on these potential surfaces, modeled after two states of the organic molecule butatriene, shows that the upper-state population substantially decays to the lower state in only $10 \mathrm{fs}$, but partially returns after about $40 \mathrm{fs}$. There is strong coupling between

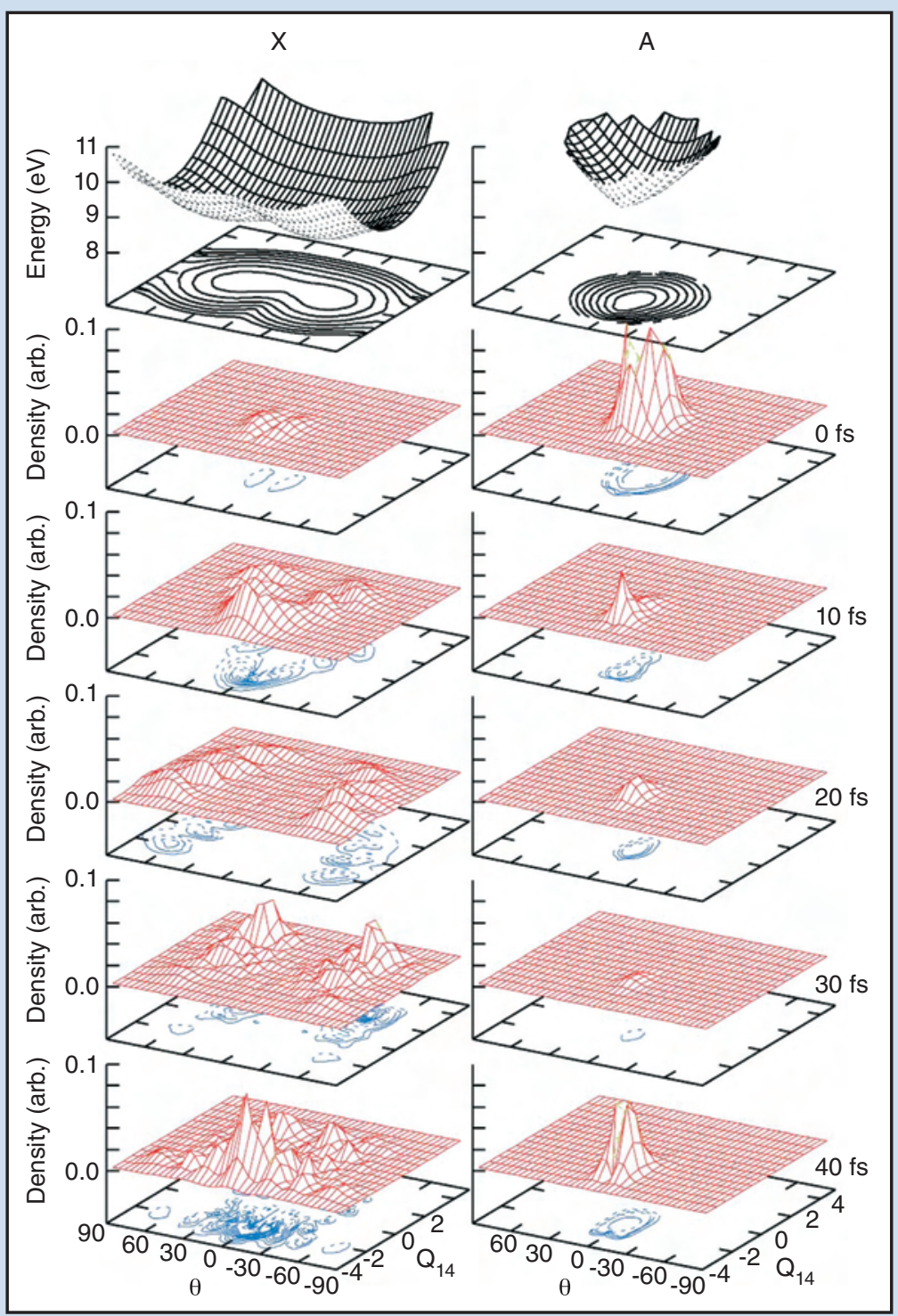

the nuclear and electronic motion in the molecule at the crossing point, signaling the breakdown of the Born-Oppenheimer approximation.

FIGURE 1. Evolution of the population of two excited states calculated from the conical intersection of their respective potential-energy surfaces (PES), which are shown in the top row. Following excitation, the upper-state (A) population decays very quickly to the lower state $(X)$ but then recovers on a femtosecond time scale. 


\section{Hot Stuff}

At the other temperature extreme lie plasmas, where thermal energies dominate (Figure 2.5). Here too, the physics has a kind of simplicity, because the particles in hot plasmas follow the laws of classical physics, which makes the computational task of predicting-or controlling - their behavior far simpler (see Sidebar "Theoretical Challenges of Quantum Calculations"). Modern examples of classical control for hot plasmas include laser-driven plasma accelerators, which are technically challenging machines and also have numerous long-term practical prospects in areas like imaging. Some quite novel and intriguing challenges, however, lie outside these traditional regimes of matter.

\section{The Transition from Solid to Plasma:}

\section{Warm Dense Matter}

The regime between hot and cold dense matter is extremely important because it is where much of the physics and chemistry of planetary interiors takes place, where the Fermi temperature, binding energy, and thermal energy are all comparable, and where theory has been so difficult that predictions have been virtually impossible. Quantum effects are important but not dominant. Phase transitions exist, but the phases and transition temperatures are unpredictable. In the next half century, the scientific community is poised to tackle this regime, owing to a new palette of computational and experimental tools. Some of these tools are

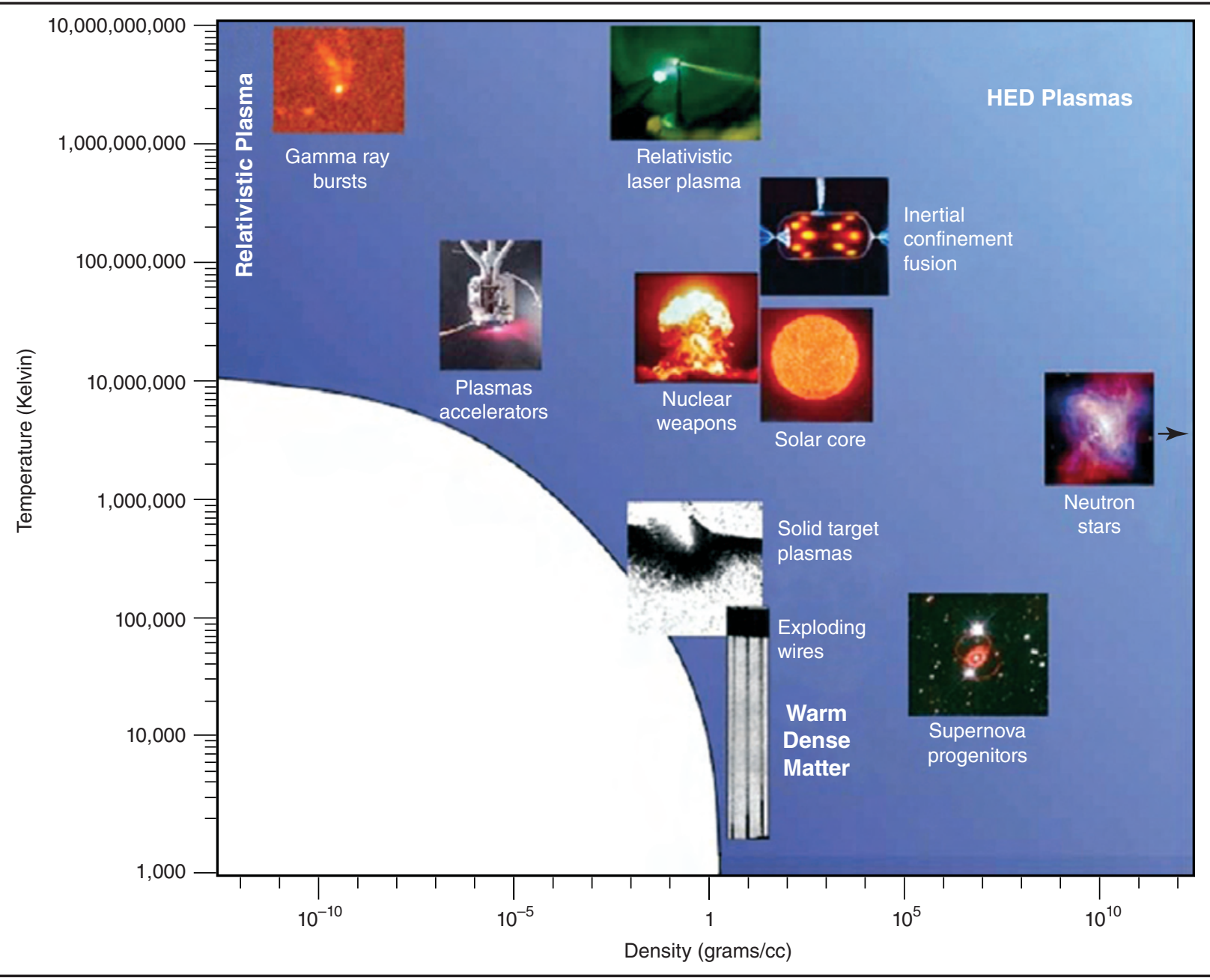

FIGURE 2.5. The space of high-energy-density plasmas. 
discussed in Chapter 7. They include advanced computation and new bright light sources such as the next-generation $\mathrm{x}$-ray free-electron lasers.

The expression "warm dense matter" suggests an interplay of different physical regimes that may be explored and controlled. High density is associated with degeneracy, correlation, ordering effects, and strong, multiple interactions; high temperature ("warmth") with ideality, thermal and spatial averaging, and the separability of degrees of freedom. Can elements of both regimes be present? Are there conditions under which they compete? If so, we can expect new physics that cannot be predicted by current condensed-matter or plasma theoretical models.

The archetypal thought experiment is to deposit enough energy in an ordered solid-state system to cause melting and ionization in a time short compared to the time scales for these processes, and then probe properties of the system as it relaxes to a plasma phase. Thus, the physics is intrinsically non-equilibrium (the focus of Chapter 6). Questions abound: what are the mechanisms of energy absorption and transfer? Are there metastable states? What, if any, is the effect of the initial-state physics? Until quite recently, the combination of ultra-fast time scales, high energy densities, and high-resolution precision diagnostics involved in these kinds of experiments was an obstacle to exploring the regime of warm dense matter. Recently, ultra-fast diagnostics and high-energy, short-pulse lasers have been developed to the point that they can start to be used to make precision measurements on well-characterized samples of warm dense matter. In the near future, fourth-generation light sources may provide a capability to probe the structure of matter at the boundary between solid and plasma with the same precision that current third-generation sources provide for the multiple solid phases of condensed systems at much lower energy densities.

\section{Cold Quantum-Degenerate Gases: Quantum Simulators, Quantum Computers, and Maybe More}

At the other extreme from the warm dense matter frontier lies the regime of ultra-cold matter. At low temperatures, the interactions that control the properties of matter are maintained in collective quantum phenomena, and the study of these fascinating systems has been an enduring challenge.

The basic particles of the quantum world have very different statistical properties depending on whether they have a total spin that is an integer multiple (bosons) of Planck's constant h divided by $2 \pi$, or $1 / 2$ integer (fermions). Electrons are fermions that have a spin of $1 / 2$ and are the basis for the room-temperature properties of atoms, molecules, and solids. All of the familiar stable constituents of atoms are fermions: electrons, protons, and neutrons. In fact, one of the fermionic properties is stability, or more correctly, conservation of total particle number. We can make stable bosonic particles with integer multiples of spin by combining fermions together; for example, the hydrogen atom-one electron plus one proton-is a stable atom with integer spin. Bosons at low temperature behave completely differently from fermions. An ultracold ensemble of hydrogen atoms can condense into a very special new phase of matter called a Bose-Einstein condensate (BEC), in which all the atoms occupy the same quantum state. Fermions obey the Pauli exclusion principle, and thus they cannot form a BEC.

Bose-Einstein condensates (BECs) of atomic gases are barely a dozen years old, and the cooling of fermionic gases is still newer, but these systems are starting to evolve as powerful tools to help us understand the quantum mechanics of collective phenomena (Figure 2.6). One of the most startling phenomena in these systems is superfluidity, the property of flow without dissipation, which was known decades earlier in lowtemperature liquid helium, but never before observed in a system that could be studied so thoroughly.

Of course, just as electrons and protons can form a composite boson, ultra-cold fermions can also pair up. Bardeen-Cooper-Schrieffer (BCS) theory describes this pairing for electrons in a cold metal. The resulting composite particles are called Cooper pairs, and they display the startling and very useful property of superconductivity.

What is the difference between Bose-Einstein condensation of fermions that have paired to form bosons and BCS superconductivity of fermions that have 


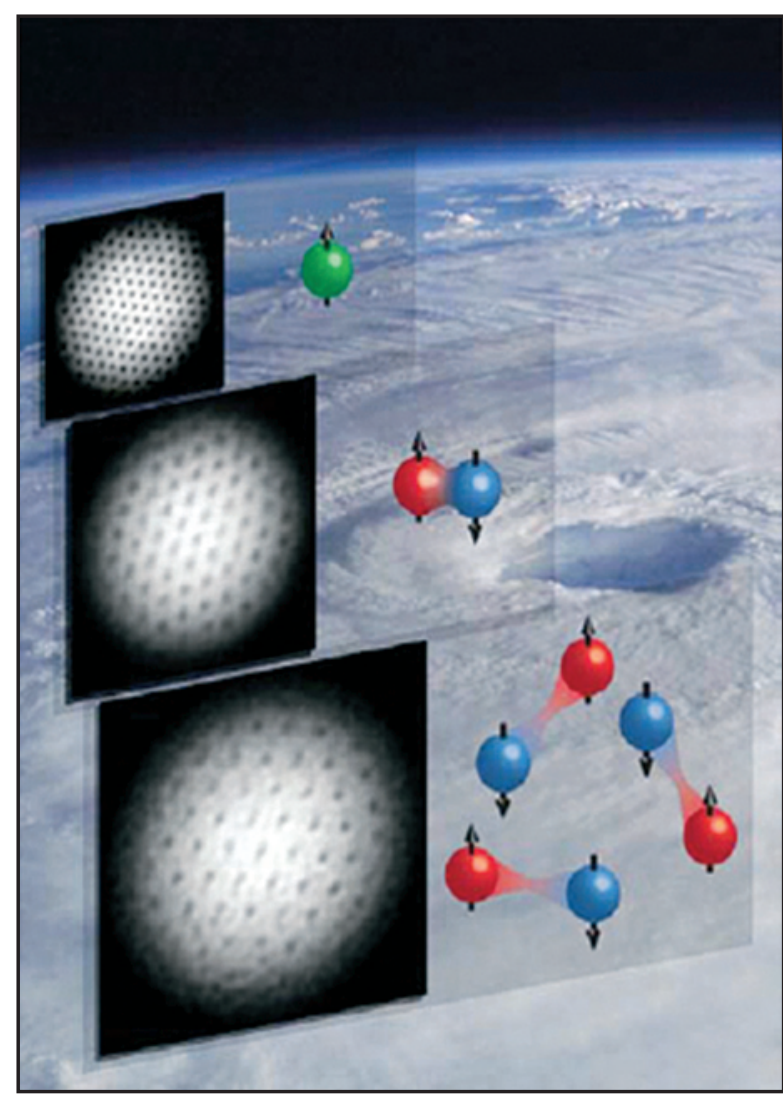

FIGURE 2.6. Superimposed on one of earth's largest vortices, a hurricane, viewed from space are three images of microscopic vortices in cooled atomic vapors.

paired to form Cooper pairs, also bosons? This is a deep question in many-body physics that has remained unaddressed by experiments until now. Evidently, the strength of the interaction determines which of these two phases describes the low temperature quantum behavior. This kind of physics may not merely address superconductivity, but it might also yield insight into more tightly bound and less well-understood fermions, such as the physics of neutron stars or the quark-gluon plasma that may have dominated the universe at an early stage of its existence (see Sidebar "Quarks").

\section{COHERENT INTERACTIONS BETWEEN LIGHT AND MATTER}

Lasers can now produce coherent radiation with almost any field strength, frequency, temporal shape, bandwidth, and polarization. Coherent matter waves have been produced and manipulated in laboratories. Engineering and controlling coherent quantum states have become a reality. Such coherent interactions are poised to open new scientific directions. The challenges outlined in the first half of the chapter-electronic states, dynamics, correlation, and exotic states of matter-could, in the future, be joined by the still greater challenge of direct coherent control by lasers. In the next few paragraphs we will describe this vision and extend it still further: to shorter wavelengths (Angstroms), to shorter times (attoseconds), to higher field strengths (thousands of times the atomic binding of hydrogen), and to ever greater complexity. The intellectual pay-off of this field will be vast because essentially all dynamical events in chemistry and materials science start at the atomic and molecular scale that could be controlled by lasers.

\section{Optimal Control and Learning Loops}

Direct laser control of chemical bonds is usually difficult because energy is transferred among all the bonds in a molecule much faster than a laser can deposit it, so that, in many cases, the laser is little more than a directed heat source. A new approach to quantum control is based on multi-path quantum interference. In quantum mechanics, there are often multiple paths that a system can take between its initial and final states. According to the laws of quantum mechanics, a quantum system will evolve on all these paths at once, and they interfere. It is similar to the sound wave interference you can hear as beating between two musical tones with different pitch, except that in quantum mechanics, a single system can interfere with itself as long as we do not know which of the quantum paths was taken. If we can externally control the quantum paths, then we can control the quantum interference, and therefore control the outcome of the physical or chemical process. We achieve this using coherent laser light to push the quantum system onto desired paths.

To use quantum control, we need to select the qualities of the laser light-its frequency, intensity, direction, polarization, and duration - to achieve a particular goal. But how do we do this? Knowledge of the optimal pulse shape requires that we invert the quantum dynamical equations, and these are calculations that are beyond present-day computers for most systems (See Sidebar "Theoretical Challenges of Quantum Calculations”). 


\section{QUARKS}

\section{The Structure of Nuclear Matter: Another Theoretical Problem Unraveled by Cold Atoms?}

Exquisite experimental control over cold atoms has now been used to demonstrate many exotic quantum properties, including superfluidity, superconductivity, Bose-Einstein condensation, BCS pairing, and many other properties of strongly correlated solids. But this may not be the limit of their power. By tuning interactions in cold atoms, perhaps we can simulate nuclear matter. Although extremely speculative, a number of researchers have begun to consider this connection.

From protons to neutron stars, nuclear matter dominates the observable mass of the universe, but we just do not know how it works because the interactions are too strong to calculate using known techniques. What we do know is that nuclei are made of baryons, and baryons contain spin- $1 / 2$ particles called quarks. These quarks, and the gluons that seem to bind them together, have some very unusual properties. For one thing, they never appear alone-evereven when kicked as hard as the highest-energy particle accelerator can kick them. They also like to congregate in twos (mesons) and threes (baryons). Elegant theories like quantum chromodynamics state that these properties devolve from a hidden internal quantum number, called "color." But these theories present extreme calculational difficulties, so that our predictive power is limited, and thus our insights into nuclear processes in neutron stars or the early universe, or even in fusion in the laboratory, is that much harder.

The DOE has been at the forefront of these problems and has built specialized accelerators to explore the properties of nuclear matter, but clearly other approaches are also needed to speed progress on these fundamental problems. We cannot place a neutron star in a laboratory, but one tremendously promising avenue that comes directly from the study and control of cold atoms is the quantum simulator. The ultra-cold atomic gas, we are coming to realize, is a many-body quantum system whose properties we can adjust with laboratory knobs and whose quantum evolution can be plotted with a good microscope and some table-top lasers.

In the ultra-cold atomic quantum simulator of nuclear matter, three different kinds of spin-1/2 atoms can represent three different quarks. The attraction or repulsion between them can be controlled by lasers and static fields. The "quarks" can be placed in regular lattices built from interfering laser beams, mirroring a simplification that is often used to make progress in quantum-field theory,

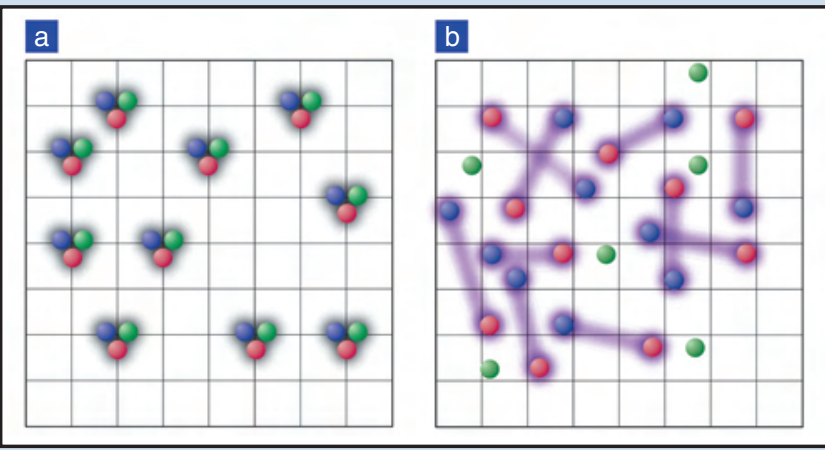
and they can be forced to congregate in threes, like baryons, or to wander freely with weak correlations, as "color" superconductors (Figure 1).

FIGURE 1. Three different kinds of atoms can form an analog to a three-quark nucleon (left) or a gas of Cooper pairs (right). 
A procedure that has been found for quantum control is to let the quantum system find its own solution through a process of adaptive learning (Figure 2.7). This learning feedback approach employs thousands of different trial laser pulses, each attempting to control the system. The results of these imperfect control trials can be used to train the laser to find the optimal conditions for control — and at the same time, teach the scientists how to control the system. This has worked in some simple photochemical systems and has even been applied to photosynthesis.

Quantum-learning algorithms and quantum computing are challenging examples of how to apply the rules of nature on the quantum scale to solve some of our most difficult chemical, materials, and information problems. The largest questions of control are unanswered: What are the general principles of control in quantum systems? How far will we go in achieving control over nature in this new way?

\section{CONTROL PUSHED TO HIGHER ENERGY, BROADER BANDWIDTH, AND SHORTER TIMES}

Today, we have unprecedented control over optical fields, which has opened unprecedented opportunities for precision measurements that can in many cases go considerably beyond the control of quantum-wave phenomena in molecules. The femtosecond-comb, which combines ultra-fast and ultra-stable laser technologies, earned a Nobel Prize for its inventors John Hall and Ted Haensch in 2005, but its real payoffs are certainly yet to come. We have already mastered important terrestrial tasks, such as knowing where we are on the earth through global positioning satellites (GPS). New challenges are tied to where we are in the universe, both in space and time. There are plenty of hints that new and possibly revolutionary knowledge awaits us just beyond our present ability to measure with precision. Challenges include the fundamental symmetries of nature revealed in electric-dipole moments, gravitational waves detected in earthbound observatories that could tell us about cataclysmic events in the universe, and the nature of the dark energy, the existence of which we only discovered in the last decade, that makes up most of the universe.

\section{Attosecond Science}

The time scale for the dynamics of electrons in atoms is femtoseconds or less. The atomic unit of time is $\tau_{\text {atomic }} \sim 24$ as. In the old Bohr theory of the atom, this is the time that it takes for a Bohr electron to complete one radian of its orbit in the ground state of hydrogen. Thus, attosecond science is fundamentally the science of electrons in atoms and molecules, and attosecond techniques may be capable of tracking electron dynamics inside atoms and molecules or on the atomic scale in condensed or solid matter. The kinds of fundamental science that might be advanced with attosecond resolution include: non-radiative chemical

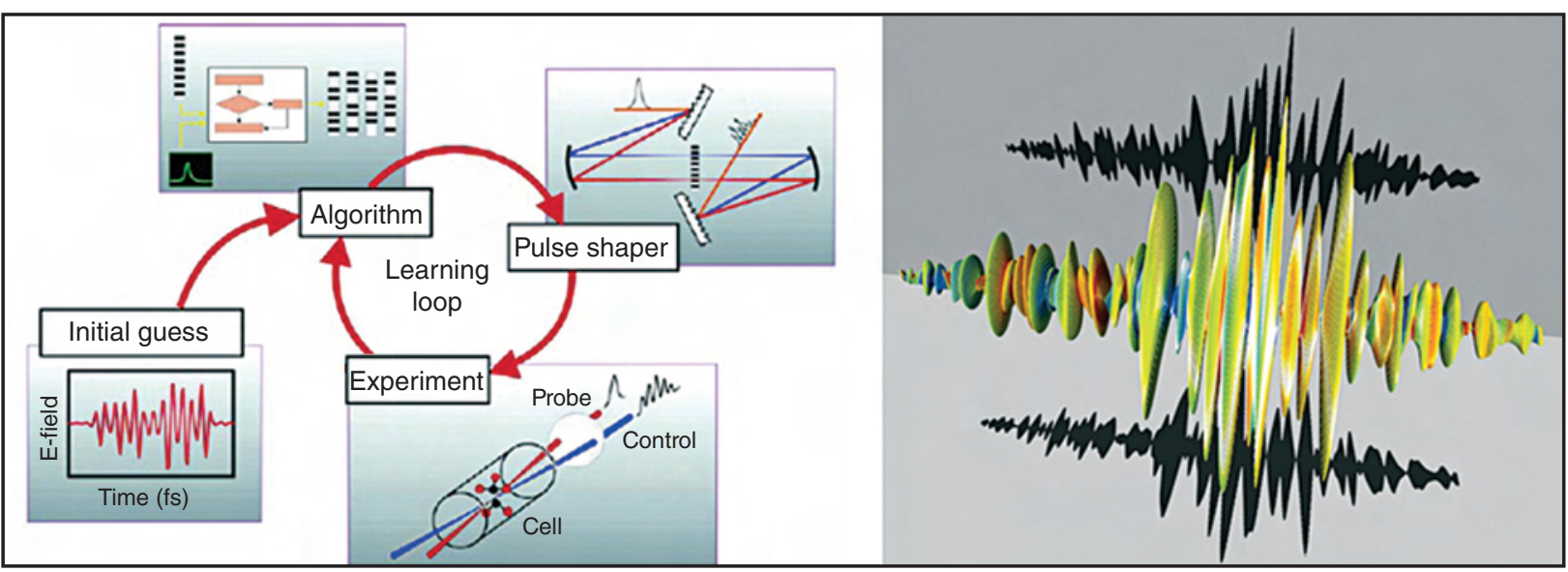

FIGURE 2.7. Left: Box diagram of a learning algorithm. The computer suggests pulse shapes, which then execute experiments. The analysis of the experiments leads to improved suggestions for pulse shapes and so on until the target is achieved. Right: Light pulses can be controlled in amplitude, color, polarization, and phase. This figure displays the electric field of a complex polarization-shaped laser pulse. 
transformation, electronic coherences in atoms and molecules discussed earlier in this chapter, and the physics of inner electronic shells.

Attosecond-scale phenomena have been studied indirectly for years by using the Heisenberg uncertainty principle to infer decay times by observing the energy uncertainty of the decay debris. Attosecond precision has often been obtained in this way, particularly in particle physics. (For example, the lifetime of the neutral pi-meson has a "measured" value of $82.0 \pm 2.4$ as.) Such inferred measurements cannot reveal many details that must exist in more complex phenomena such as the relaxation of core-excited molecules, so that there is really no substitute for direct observation.

Lasers have been developed over the past two decades that have special features allowing extremely short optical pulses, which could be used to take snapshot images of ultra-fast processes. These laser-based techniques are still limited by the modulation speed of the light, which cannot be faster than one optical period, or about 2 fs for red light. A single optical cycle seemed until recently to be a natural lower bound on direct measurements of short intervals of time.

\section{Attosecond-Pulse Generation Requires}

\section{New Technology}

Optical light waves oscillate with a period of 1-2 fs, and so attosecond pulses are vacuum ultraviolet or soft $\mathrm{x}$ rays. Right now, we can make optical pulses about 4.5 -fs duration, or just over one optical cycle. The methods to do this use nonlinear optical principles that are well known and have been commercialized for more than a decade.
The recent leap to sub-femtosecond pulses required a breakthrough technology, which has come from the implementation of the realization that the shortest "pulse" that we could imagine is the inelastic collision between two point-like particles. For example (Figure 2.8), if we focus an ultra-fast laser pulse into an atomic gas, the electric field at the focus rips electrons out of every atom, which then re-collide with their parent ions with sub-cycle precision. Under the right conditions, a narrow beam of vacuum ultraviolet or soft $\mathrm{x}$-ray radiation is generated with a duration as short as 200 as, or less.

\section{Using Attosecond Pulses}

Just as important as the generation of attosecond pulses is the means to propagate, characterize, and use them productively. The attosecond generation process shown in Figure 2.8 has also been employed to take ultrafast images of electrons in atoms (see Sidebar "Quantum Electron Scattering"). In the future, we may be able to record the dynamics of electrons as they undergo transitions between atomic orbitals-and even follow the correlated dynamics of multi-electron motion in molecules.

An important example of an attosecond process is Auger relaxation (Figure 2.9), which occurs when $\mathrm{x}$ rays ionize multi-electron atoms. Unlike normal photoionization with visible or $\mathrm{UV}$ light, $\mathrm{x}$ rays remove a deeply bound electron. This creates an atom with a vacancy in an inner electron shell that must relax through subsequent dynamics of at least two electrons from a more weakly bound outer shell. The result is that the atom transforms, in less than one femtosecond, from a neutral initial state to a doubly charged ion.

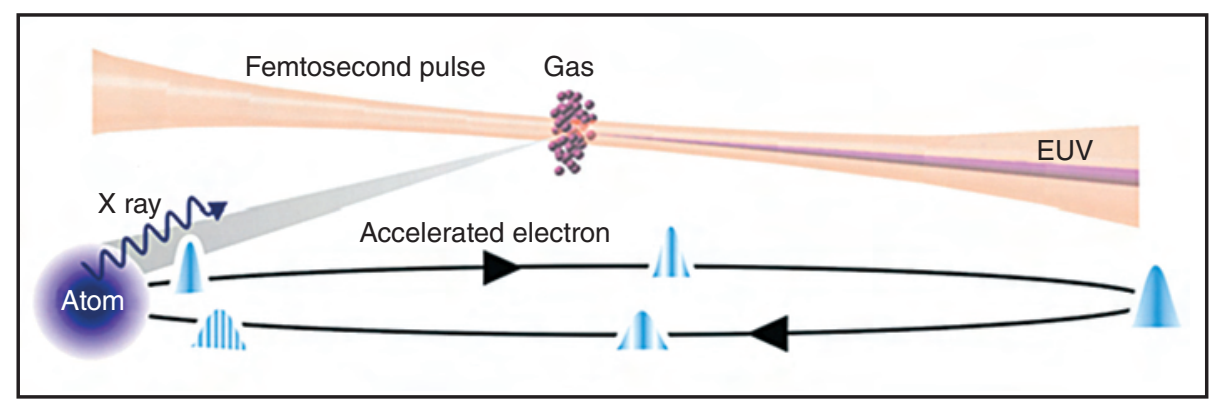

FIGURE 2.8. In the center of a tightly focused laser beam, the electric fields can easily exceed the atomic binding force. Electrons are literally ripped out of atoms by the laser field and then driven back to recollide with their parent atom in a femtosecond or less. The recollision produces a burst of attosecond $\mathrm{x}$-ray radiation. 


\section{QUANTUM ELECTRON \\ SCATTERING}

In this chapter, we have described a new approach to control the quantum world that bypasses our woefully inadequate supercomputers by asking the quantum system to help us find the control solutions. A similar approach may help us with imaging on the quantum scale by asking the atom or molecule to image itself. The advantage here is that the quantum system has access to quantum-phase information that is hidden from classical measurement tools.

Quantum imaging has already been developed for simple molecules. For example, if an intense femtosecond laser is focused onto a molecule, an electron may be field-ionized and then recollide a half-optical cycle later. This electron becomes the probe to interrogate its parent molecule. The electron can scatter coherently from the molecule or may be captured in the process that produces high harmonics. Either way, the emitted photon or electron will provide phase-coherent information on the electronic structure of the molecule.
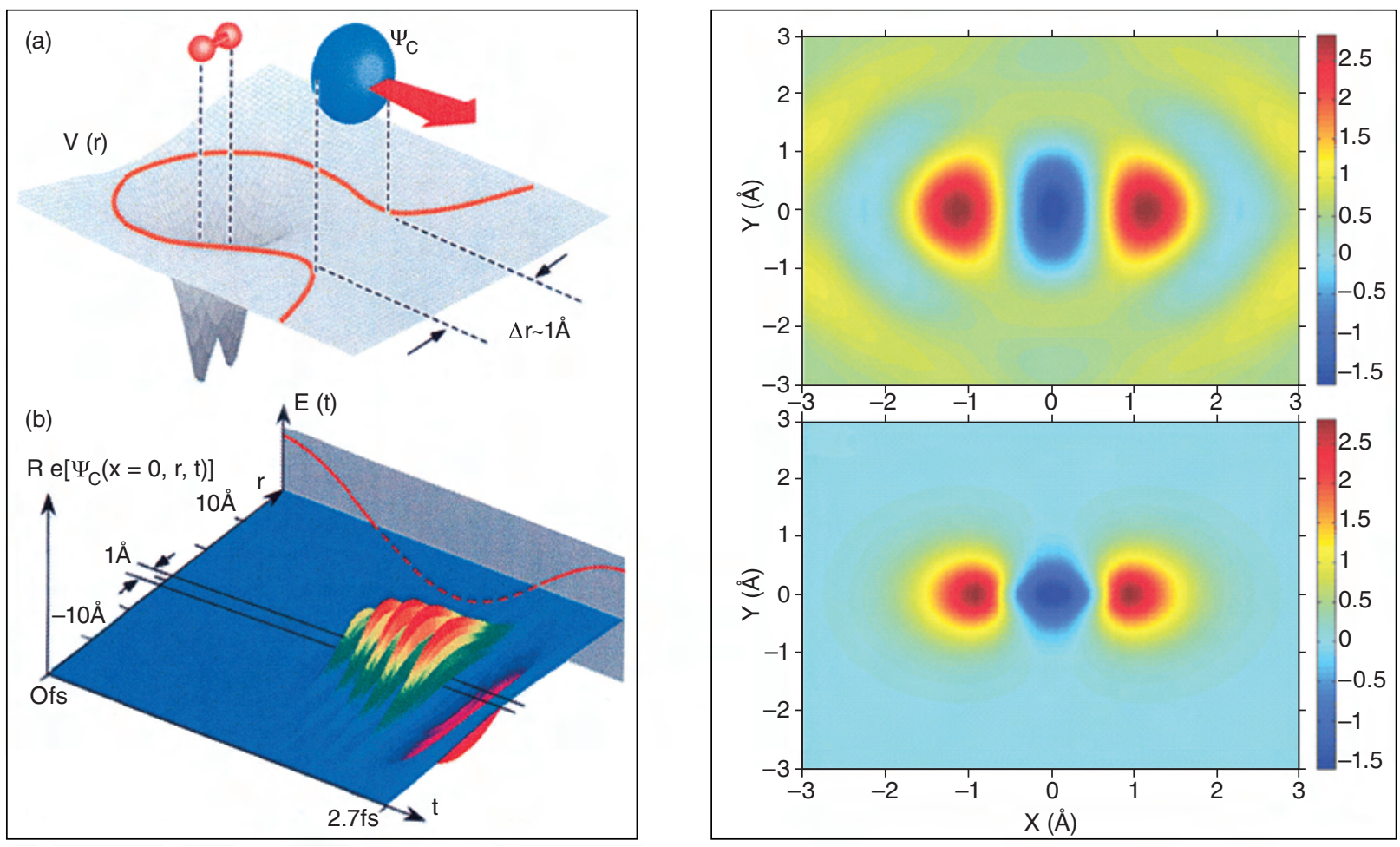

FIGURE 1. Left: Cartoon showing collection of an image of a nitrogen molecule as it undergoes strong-field ionization and recollision. Right: The image collected from the radiation produced in the recollision (top) and a calculation of the most loosely bound ground state electron in nitrogen (bottom). 


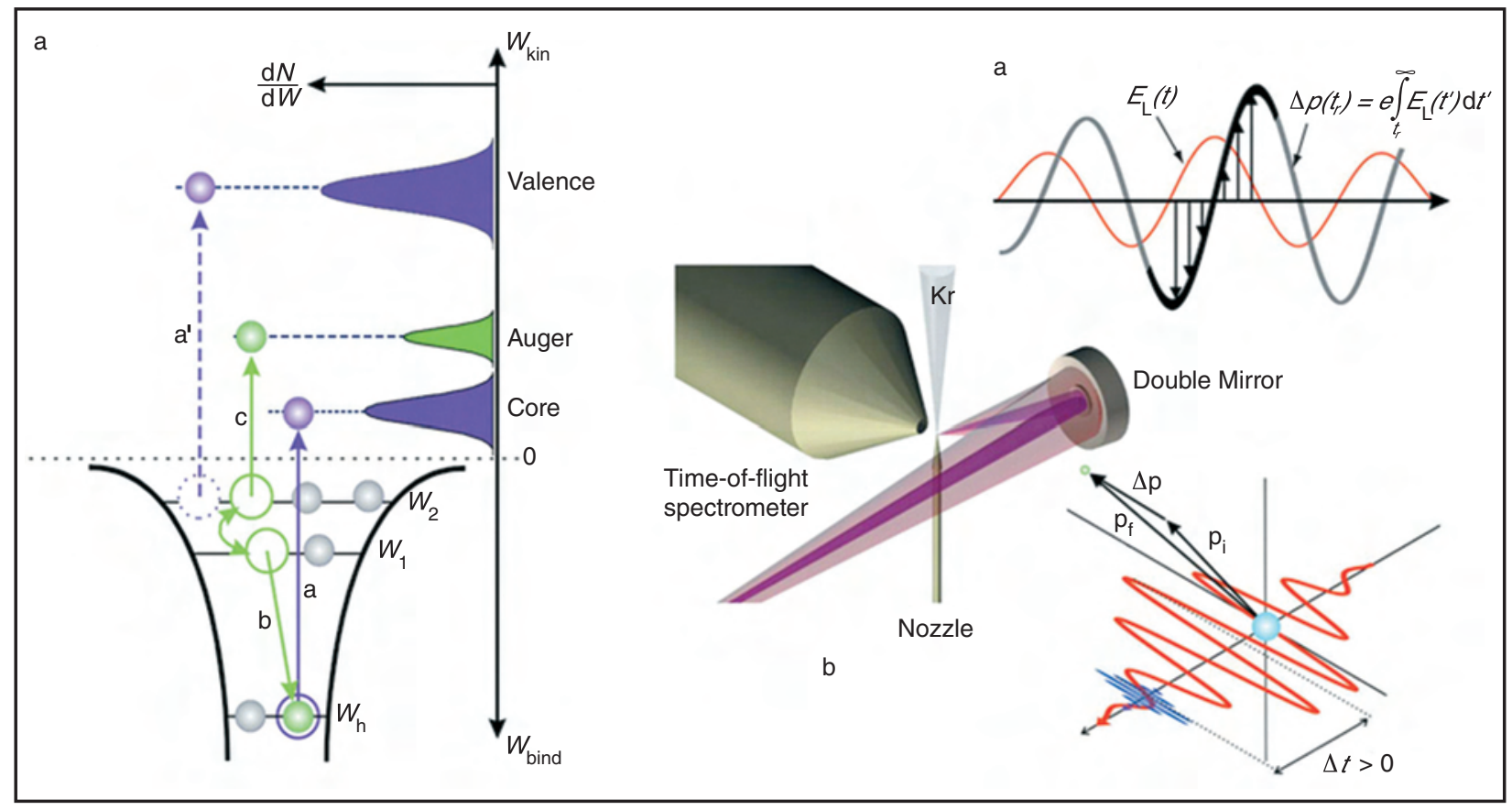

FIGURE 2.9. Left: In the Auger process, an $\mathrm{x}$ ray photoionizes a tightly bound electron from an inner shell, and then an outer electron fills the vacancy while a second outer electron is ejected. Right: The delay of the Auger electron following the initial photoionization can be measured by using the strong oscillating field of the laser to deflect the electron.

Attosecond pulses can capture this process as it evolves, on its own natural time scale.

\section{$X$-Ray Laser Science}

$\mathrm{X}$-ray synchrotron sources are a major $20^{\text {th }}$ century success story for BES and have made routine the field of protein crystallography studies and aided electronic structural studies in atomic, molecular, and solid-state science. The pace of work done with these sources continues to accelerate today, and the payoffs accumulate.

A new generation of $\mathrm{x}$-ray sources with brightness a billion times higher than the brightest synchrotrons is about to go into operation in the U.S., Europe, and Japan (see Sidebar "Four Generations of Light Sources" in Chapter 7). If revolutions can ever be predicted in science, a billion-fold increase in the brightness of a light source would seem to be a good bet. These new sources allow accelerator-based technology to have the coherent properties of lasers.

Free-electron lasers (FELs) are expensive investments compared to table-top lasers, but the return in terms of discovery science and follow-on applications is expected to be substantial. Much has already been written about their capabilities for new applications in BES research. The most important new qualitative features compared to existing $\mathrm{x}$-ray sources are directly connected to the laser-like qualities of the beams: the short pulse duration and the high field strength.

Pulse duration. The nominal pulse duration for the early operation of x-ray FELs (XFELs) is on the order of $100 \mathrm{fs}$. The minimum pulse duration based on the technology is in the range of 200 as, and there are enough ideas about how to reach this that there is little doubt that it will be achieved; therefore, the attosecond research program described above will have an opportunity to take advantage of these machines.

Field strength. X-ray lasers are capable of focused intensity in the range of $10^{20} \mathrm{~W} / \mathrm{cm}^{2}$ or more, simply by focusing these coherent beams to spot sizes of a tenth micron or less. This is sufficiently high that even tightly bound core electrons in atoms will be distorted or field-ionized by the strength of the field. The ramifications of this field strength for physics of atoms, molecules, and materials are not yet fully understood, but there will be a significant impact on high-energydensity plasma physics and related areas. 
Even the fundamental mechanisms of damage at such high intensities are not well understood. Are there important new nonlinear damage mechanisms such as $\mathrm{x}$-ray field ionization or multiple core-hole formation? Can the coherence of the $\mathrm{x}$-ray laser change the character of the damage? Can we find ways to lessen the effects of radiation damage on imaging by shortening the duration of the laser pulse or by changing the properties of the beam?

\section{THE HUGE CHALLENGES OF COMPUTING AND INFORMATION}

The last challenge we cover in this chapter is perhaps the greatest: the challenge of computing and information. The quantum simulations discussed earlier in this chapter are a form of physical computer with which we can compute the state or the evolution of a physical system. Our familiar digital computers can also do this, but they are limited by size and speed. For three decades now, we have become accustomed to the ever increasing power of our computers. But what lies beyond Moore's Law? As any computer-savvy teenager will tell you, Moore's law is the prediction made by Intel co-founder Gordon E. Moore in 1965 that the number of transistors on a chip would double every two years (Figure 2.10).

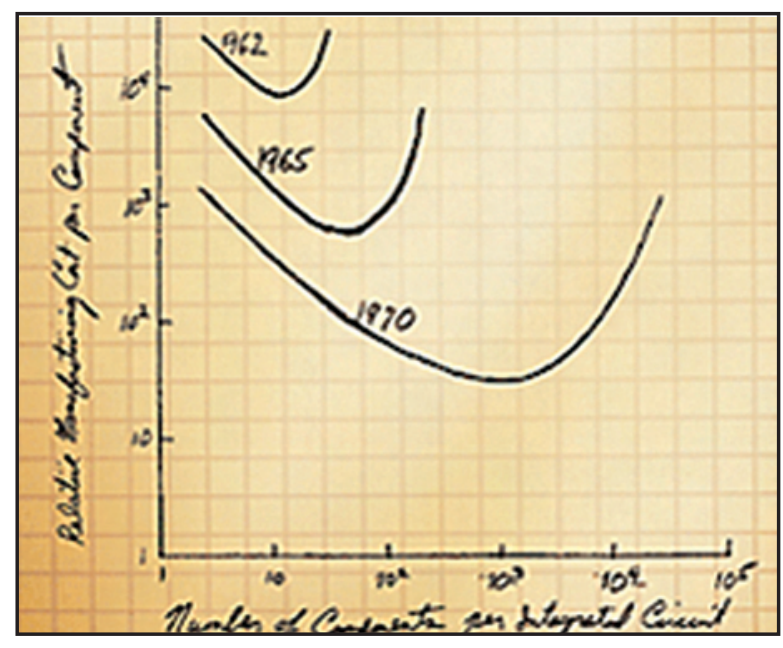

FIGURE 2.10. Moore's original 1965 graph showing the cost per transistor as a function of the number of transistors on a chip. Moore predicted that the number of transistors at the curve minimum, which represents the most efficient chip capacity, would double every two years.
Moore's Law is close to its natural end. The most efficient chip capacity will soon stop doubling every two years. The reason is simply that the classical laws of physics that have governed computer hardware and the laws of binary logic that dominate computer software have finally run out of room. Smaller transistors are harder to fabricate as the feature size on a chip drops to nearly an order of magnitude smaller than the wavelength of visible light and as the "gate oxide" which controls current flow in a transistor reaches thicknesses of a few atoms, so that electrons can undergo unwanted tunneling across the gap. (See also the Sidebar "Defectology and the End of Moore's Law" in Chapter 5.) Power consumption and cooling are also limiting factors. If we look inside a desktop computer today, the most obvious elements taking up space are the heat sinks and fans.

Nonetheless, we need more computer power. Quantum calculations of phenomena described in this chapter already outstrip the capabilities of the most powerful computers (see Sidebar "Theoretical Challenges of Quantum Calculations"). The answers to this problem are far from clear, but it is likely that a revolutionary change in computing will have to occur soon in hardware (quantum computers? analog computers?) and in programming (analog programming? probabilistic adaptive programming?).

Quantum information science may or may not be the solution to this problem or for computation when Gordon Moore's law runs out of steam. Quantum calculations are based on the power of quantum mechanics, the same power that defeats classical computational tools as described above for electronic excited states. Instead of bits and bytes stored in transistors whose states must be "0" or "1," quantum information is stored in "qubits"-quantum bits whose value can be both zero and one at the same time. This is not nonsense; it is the special property of quantum mechanics called superposition. Each dimension can take on multiple values through superposition: not spin up or spin down, but both, in precisely defined amounts. Even more fascinating is the notion of "entanglement," which is correlated superposition; e.g. two spins are not only each in superpositions of up and down, but they are correlated so that if one is up, the other must be down, and vice versa (see Sidebar "Entanglement"). 


\section{CONCLUSIONS}

This chapter has covered the Grand Challenge of quantum control at the level of electrons of atoms, molecules, and aggregate materials, from ultra-cold quantum degenerate gases to $\mathrm{x}$-ray free-electron lasers.
These subjects may seem separated by enormous distances in technology and science, but they are unified by the concept of coherence and control in material systems. In the $21^{\text {st }}$ century, quantum control will become an important tool for addressing basic problems in physics, chemistry, and materials science and engineering. 


\section{THE BASIC ARCHITECTURE OF MATTER: DIRECTED ASSEMBLIES, STRUCTURE, AND PROPERTIES}

The Grand Challenge for the Basic Architecture of Matter is to design and perfect atom- and energyefficient syntheses of revolutionary new forms of matter with tailored properties.

Humankind's ability to create and manipulate the properties of natural and synthetic materials has marked much of the progress made by our civilization. Indeed, the terminologies "Stone Age," "Bonze Age," and "Iron Age" denote our increasing mastery over these materials. In the new era that science now enters, the emerging mastery of the ability to control energy and matter at the most fundamental level will permit the creation of new materials with properties tailored to meet the changing needs of our world.

In early times, the manipulation of matter was largely derived from empirical, trial-and-error observations. The $19^{\text {th }}$ and $20^{\text {th }}$ centuries saw dramatic progress toward an ability to create matter rationally as an experimental and theoretical understanding of atoms, molecules, and solids emerged. The discovery of new substances using increasingly sophisticated synthetic and processing methodologies progressed hand-inhand with the scientific ability to define ever more precisely the arrangements of atoms and electrons in matter and the transformations that they undergo, along with the theory to explain and predict these phenomena. It is useful for purposes of discussion to classify matter generally in terms of "hard" and "soft," terms that convey a qualitative sense of mechanical characteristics at various length scales. In a complementary fashion, one can classify matter as "organic" and "inorganic," with the former referring to substances (many of which are molecular) based largely on the elements carbon, nitrogen, oxygen, and hydrogen $(\mathrm{C}, \mathrm{N}, \mathrm{O}$, and $\mathrm{H})$, and the latter referring to nearly everything else, largely consisting of extended structures. Of course many substances fall between these rough categorizations, and they are sometimes the most interesting.
Despite the scientific advances made to date, horizons of great discovery and knowledge await scientists working on materials design and synthesis in the $21^{\text {st }}$ Century. The periodic table contains more than $110 \mathrm{el}-$ ements, but only a tiny fraction of all possible chemical compounds has yet been prepared and their properties characterized. Moving beyond trial-and-error searching, science is in favorable cases now approaching the threshold of "directed" synthesis guided by predictive design, based on first-principles, of materials with properties that we desire. How mechanically strong can we make a substance while keeping it light-weight? How durable? How resistant to corrosion, decay, or failure in extreme conditions of temperature, radiation, or enviornmental exposure? How electrically and thermally conductive? How transparent? How small yet functional? How biocompatible? Can we learn from nature (and then go beyond!) how to make substances that repair themselves? That even replicate themselves? That constantly modify themselves in response to changing environment? Finally and operationally most important, what new techniques and strategies are needed to arrange atoms into remarkable new substances? These are questions we cannot answer now, and they set the stage for a Grand Challenge of Matter in the $21^{\text {st }}$ Century.

\section{MOTIVATION FOR THE GRAND CHALLENGE OF MATTER}

The emerging mastery in control of matter and energy sets the stage for this Grand Challenge. The creation of new forms of matter is predicated on chemical reactions involving the rearrangement of atoms. Chapter 2 describes reactions as the making and breaking of bonds and the redistribution of the electrons that are associated with them. The control of matter, at the most fundamental level, involves directing these electrons during the "ungluing" and "regluing" of 
atoms. Scientists have not yet been able to observe atoms changing their partners in real time.

The new light sources of Chapter 7 and the time scales accessed with the developments of attosecond lasers in Chapter 2 will allow the synthesizer of new materials to view a "movie" of a reaction at the atomic scale in real time. A reaction plunges atoms into a fiercely competitive environment. An astronomical number of atoms are ungluing and re-gluing over the course of a reaction. By having available movies of reactions in real time, we can define the rule sets for this competition and in doing so direct which partners decouple and couple - a process known as selectivity. The quantum coherence methods of Chapter 2 will also allow us to understand how atoms, which might not otherwise want to change partners, are able to do just that, in the regime far-from-equilibrium that Chapter 6 seeks to understand. In this way, the materials designer will be able to direct a symphony, a discordant one that the rules of equilibrium would normally make inaudible. Using the quantum coherence, we will be able to understand how to make bonds break and make those that do not wish to. In arriving at an understanding of how to direct this non-equilibrium reaction chemistry, we will be able to synthesize materials with properties that were once unimaginable. To be fruitful, such efforts will also require a better understanding of matter far from equilibrium as outlined in Chapter 6. The new synthetic capability described here will be crucial to the in-depth characterization of the exciting materials described in Chapter 4.

In the $21^{\text {st }}$ Century, advances in theoretical understanding and computational power and methodology might also turn the design rules for materials upside down. The "reverse design" of materials is the "Holy Grail." At present, we have little understanding of what it will take to achieve this. Beginning with a set of desired materials properties, the specific type of atoms and their arrangement needed to achieve the desired target materials will be defined in the computer. With this as a guidepost, the materials designer can set about to bring truly extraordinary materials to a tangible reality.

\section{SOFT AND HARD MATTER}

Advances in science are often driven by a tension between phenomena that lie at different ends of the properties spectrum. Soft and hard matter exemplify this perfectly. Soft matter has almost infinite architectural tailorability and processiblity but in general lacks robustness. In contrast, hard matter can be mechanically tough but difficult to build with soft-matter precision on the atomic scale.

As we attain control of energy and matter, the distinction between soft and hard matter may blur. Importantly, the ways soft and hard matter are designed may converge and desired materials properties will be realized, regardless of whether the material is soft or hard. Hard matter may be constructed with the atomlevel precision, versatility, and tailorability with which we now engineer much of soft-matter architecture. Soft materials might be constructed with pre-programmed durability and with electronic and mechanical properties that are now the exclusive domain of hard matter. These new capabilities can lead to the materials that humankind needs to confront the energy and environmental challenges of our new century.

\section{SOFT MATTER CHALLENGES}

While soft matter has almost infinite architectural tailorability, even its very name implies that it somehow lacks robustness. A fascinating question is whether such fragility must be intrinsic to all soft matter. For example, isolated instances exist of soft matter that is robust in various ways, ranging from industrially produced phthalocyanine dyes, ultra-high modulus polyimides such as Kevlar, ultra-temperature-resistant Nomex (Figure 3.1), to the enzymes of extremeophiles that live in volcanoes and nuclear reactors. Nevertheless, the overarching physical and chemical concepts and methodologies needed to rationally create such materials are currently at a primitive/empirical level. We know that carbon nanotubes, Kevlar, and natural spider webs have exceptional mechanical properties, but we do not understand the limits of such behavior or which 


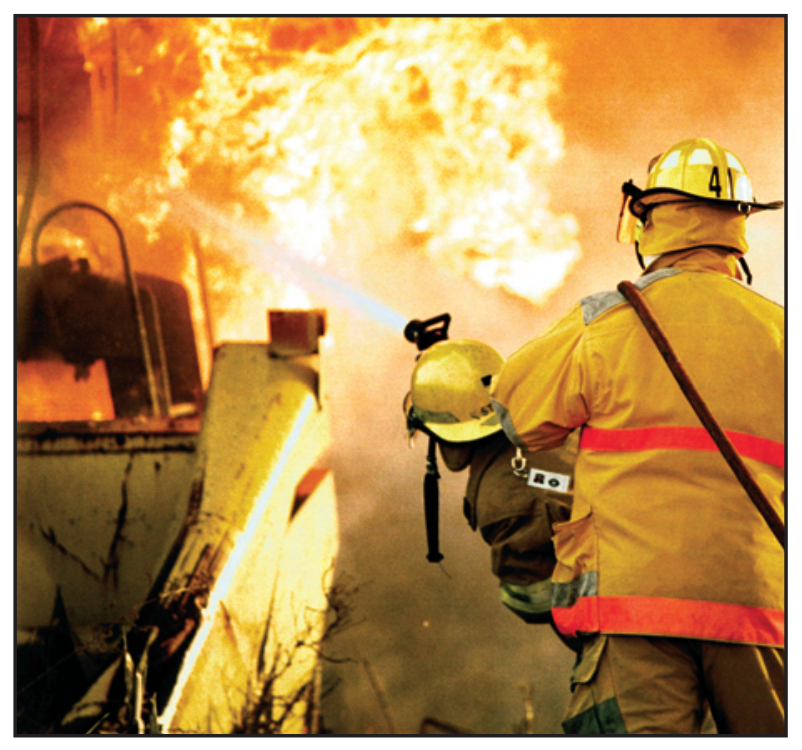

FIGURE 3.1. Used to make fire-fighters' jackets, Nomex is a human-made polyamide polymer with a structure similar to natural amino acids. It displays remarkable mechanical flexibility and strength along with flame resistance. This is exceptional behavior for soft matter. Can we gain the understanding to make it general?

modifications to make (or how to make them) to enhance and diversify these properties. The soft-matter scaffolding (ligands) that organizes and activates abiotic and biotic (enzyme) catalysts, of great importance to industrial and life processes, is typically fragile, and many of these catalysts have limited lifetimes before they undergo degradation. Nature frequently solves this problem by the remarkable self-repair process of constantly manufacturing more of the catalytic component that "wears out" and replacing it.

In most cases, we do not understand enough about the characteristics of ligand architecture and metallic electronic structure that govern catalytic activity, selectivity, and durability to allow first-principles catalyst design. A challenge would be to design and create molecular catalysts capable not only of self-repair, but of self-correction in activity and selectivity in response to a changing reaction environment or a challenging substrate. We know even less about how to construct "cascades" of coupled abiotic catalytic transformations. Imagine a system of complementary, perhaps selfcompartmentalizing synthetic catalysts that effectively couple sequences of transformations in a single reaction vessel, rather than requiring inefficient, timeconsuming initiation, work-up, and product separation from a series of transformations carried out in separate containers under different reaction conditions.

We also know today that soft matter can participate in charge transport, in both abiotic and biotic systems. Films of the oriented "doped" organic polymer polyacteylene have electrical conductivity within several orders of magnitude of that of copper metal, while natural photosynthetic systems unidirectionally transport photo-generated charge at rates approaching $10^{12} \mathrm{~s}^{-1}$. However, we are just beginning to understand the limits and subtle design principles for such behavior, much less coupling it to light absorption, light emission, or electron-hole pair generation. If we could, we could perhaps create the ultimate solar cells that not only exhibited high power-conversion efficiency, but were cheap, light weight, mechanically flexible, (Figure 3.2), manufacturable, and perhaps self-repairing and self-correcting with age or change in radiation or environment.

Below are some soft-matter challenges in both synthesis and properties, recognizing that the obvious attractions of soft matter include light weight, processability (molding, stamping, extruding, spin-coating, self-assembling), modifiable mechanical and electronic properties, great diversity and tunability in architecture and functional groups on many length scales, and exquisite possibilities for biomimicry and/or hybridization with biological systems.

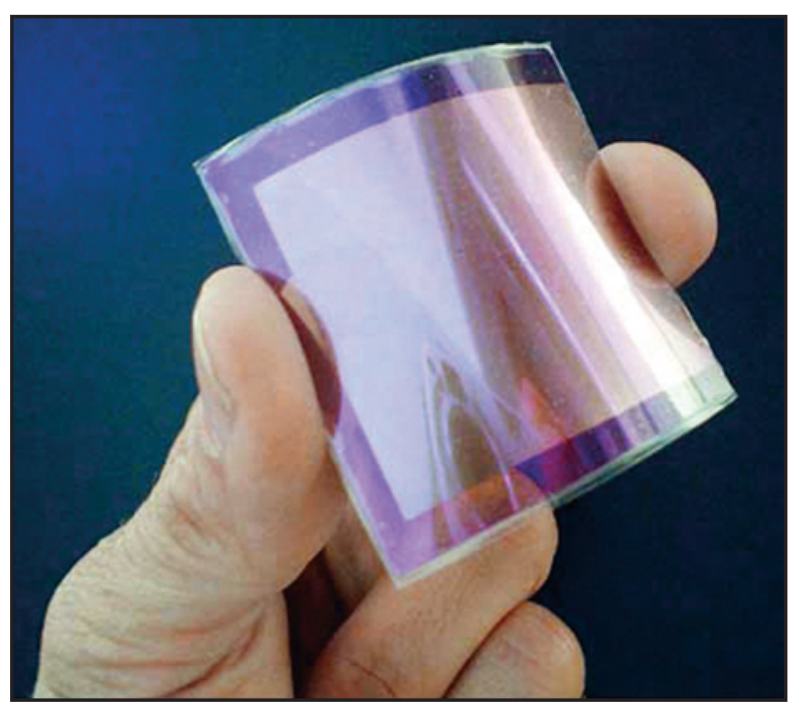

FIGURE 3.2. A flexible solar cell, the active region of which is made from polymeric soft-matter organic materials. Significant advances in materials design and synthesis will be required to achieve needed power-conversion efficiencies. 


\section{Soft-Matter Durability}

Here we desire the knowledge and design principles to understand how to create soft matter that is exceptionally durable with regard to thermal and oxidative degradation, redox cycling, and radiation damage (photon, charged particles, neutron). Achieving these goals will require a deep understanding of the degradation pathways as an integral part of the materials design process as well as new synthetic approaches. The ultimate achievement will be to create materials with the unique capability of self-healing or self-protecting (Figure 3.3) and/or with recyclability via either disassembly/reassembly or selective degradation to useful products. For an insight into how biology solves such problems, see the Sidebar "Nature's Answer to Fragile Building Blocks."

\section{Soft-Matter Architecture}

We desire the understanding to design and synthesize soft matter organized or even self-organized in three dimensions over multiple length scales. If we knew how to do this, we could achieve unprecedented control over many intriguing and useful properties.

A major challenge is to master single and multiple bond connectivity to achieve controlled electron delocalization over precise dimensions. Today our ability to create such bonding in controlled two- or three-

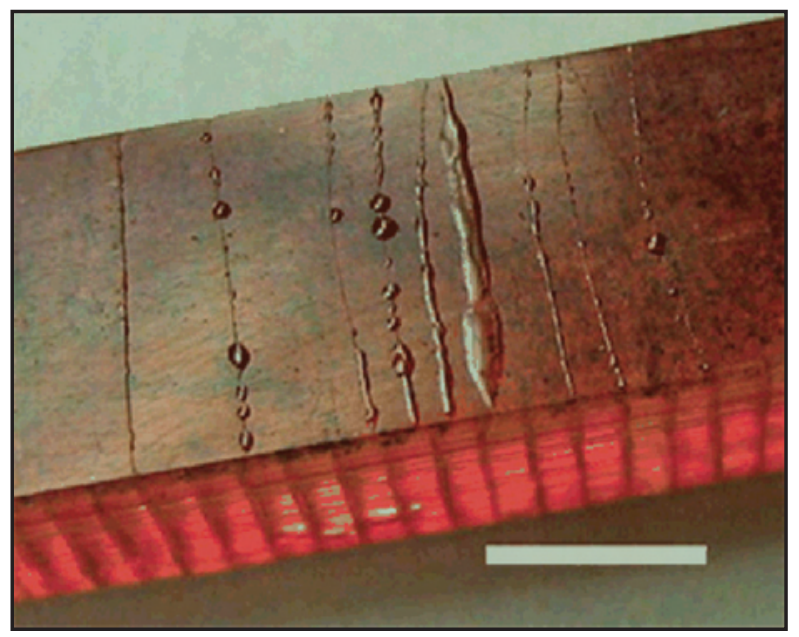

FIGURE 3.3. A self-healing polymer structure that releases a catalyst solution and a polymerizable monomer to repair cracking. The image shows the polydicyclopentadiene (polyDCPD) repair polymer forming in the crack. The scale bar is $5 \mathrm{~mm}$. dimensional connectivity is limited, and we require new synthetic methodologies to connect $\sigma$ - and $\pi$-bonds at will. Another challenge is to create cavities or protrusions of varying shapes, chiralities, functionalities, and recognition (especially biorecognition) characteristics. This could afford structures that could selectively recognize and "dock" with each other. This is a prelude to achieving far more selective catalytic transformations and better understanding enzymatic function. Achieving this goal would also allow new and/or more efficient forms of adhesion; spatial control of hydrophilicity and hydrophobicity; more effective sequestration of persistent, biocumulative, and/or toxic agents; abiotic catalysts with the properties of enzymes; and better controlled release of drugs, fertilizers, and/or active agents.

Another important problem in soft-matter design is to achieve controlled spatial organizations of electron donor and acceptor groups as well as paired and unpaired electron spins. Realizing this goal could afford new chemical reagents for selective transformations involving electron transfer, completely new types of softmatter optical, electrical, and magnetic materials, and materials for sensing, activation, and catalysis. We would also like to know how to create surfaces that reorganize in response to environment, thereby tuning compatibility or incompatibility between dissimilar materials. This knowledge is essential for developing soft matter that reorganizes to protect itself from harsh environments, but also to create soft matter that recognizes and adopts its exterior to maximize compatibility with other soft or hard matter. This could afford new types of supramolecular structures, polymer blends, and polymer composites.

The unique compatibility and incompatibility characteristics of soft materials offers unique opportunities to realize periodic microstructures that promote formation of structural-phase-separation patterns over multiple length scales. Elegant examples have been achieved with limited numbers of block copolymers (polymers having defined repeat sequences of dissimilar monomer units in the chain). We desire the synthetic capability to broaden the scope to other materials and length scales. Such structures offer intriguing opportunities to create photonic band-gap materials (those with periodic structures that guide the flow of light in ways analogous to how semiconductor lattices guide the flow 


\section{NATURE'S ANSWER TO FRAGILE BUILDING BLOCKS}

\section{Repair of Photosystem II in Green Plants}

The overall chemistry of natural photosynthesis can be written as $2 \mathrm{H}_{2} \mathrm{O}+\mathrm{CO}_{2} \stackrel{\text { light }}{\rightarrow}\left(\mathrm{CH}_{2} \mathrm{O}\right)_{n}+\mathrm{H}_{2} \mathrm{O}+\mathrm{O}_{2}$ where the $\mathrm{O}_{2}$ is generated by oxidation ("splitting") of water in Photosystem II (PSII). This reaction requires very high redox potentials of the catalytic intermediates and can produce highly reactive oxygen species. It is astonishing that such a demanding and potentially toxic reaction can occur in a delicate protein environment. It is thus not surprising that during water oxidation, protein damage occurs. In bright sunlight, Photosystem II is replaced every 30-60 minutes.

The major repair process is remarkable. One protein, called D1 (see Figure 1, top), bears the brunt of the damage, and the damaged D1 protein (light blue) is removed from the center of the PSII supercomplex by a molecular machine called Ftsh (see Figure 1, bottom), which contains 25 to 35 transmembrane and peripheral proteins. A new D1 protein is inserted into the supercomplex by a second large complex, restoring full activity to Photosystem II.

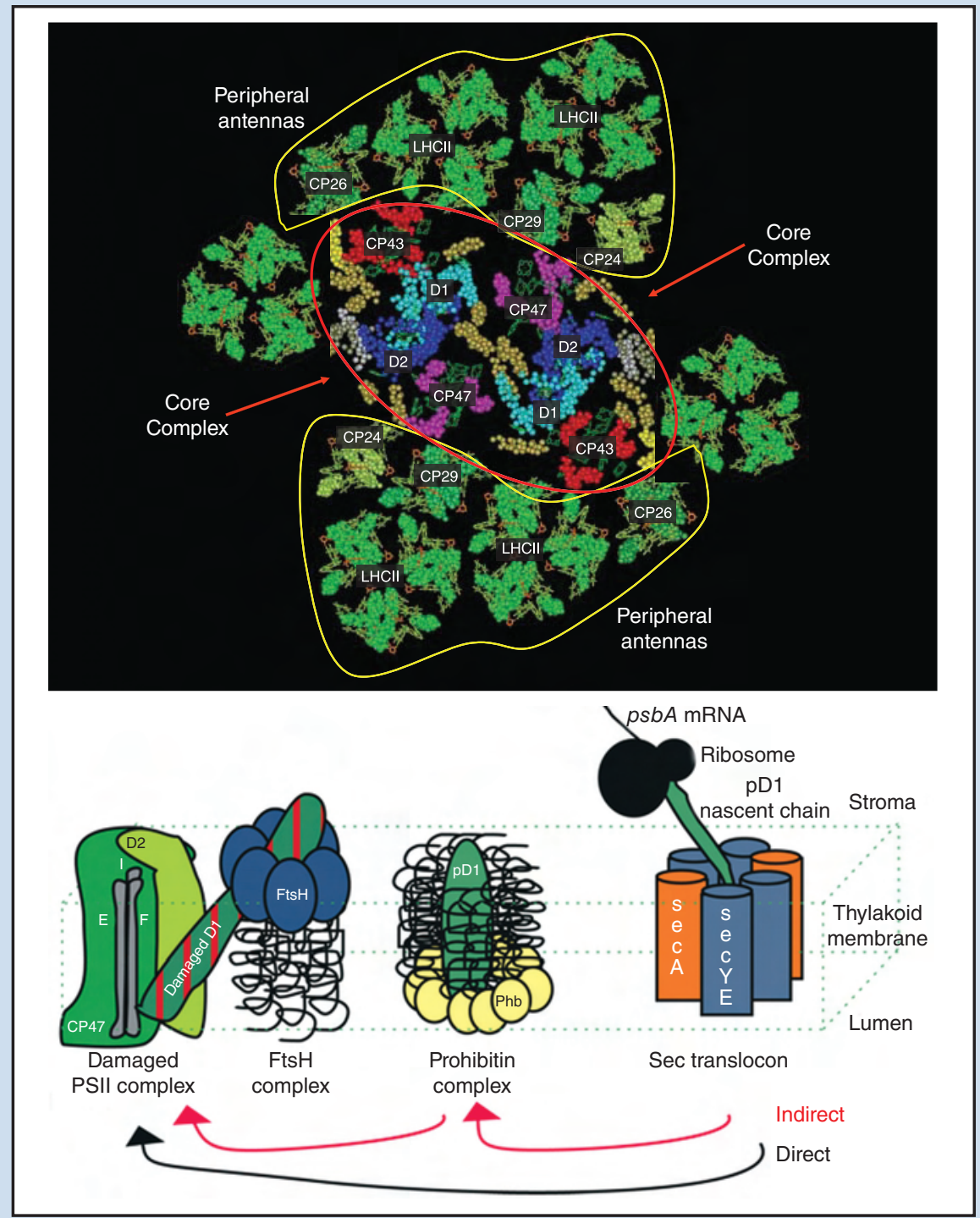

FIGURE 1. Top: View from above of the photosynthetic membrane of Photosystem II (PSII) supercomplex. Most of the pigment-protein complexes are for light harvesting, and charge separation is accomplished in the D1 and D2 proteins (light blue and dark blue, respectively) in the center of the complex. Bottom: Model of the repair process viewed in the membrane plane. Only a portion of the PSII supercomplex is sketched. 
of electrons) and serve as templates for the growth of new soft or hard-matter arrays.

\section{Some Soft-Matter Catalytic Properties}

Homogeneous catalysts are those that operate while dissolved in solution and include both synthetic molecules, which frequently contain one or more metal ions sequestered by "soft" organic ligands, and enzymes that are elaborate and elegant examples of nature's catalysts and that may or may not use metal ions at the catalytically active site (for example, compare Figures 3.4 and 3.5). Closely related are heterogeneous catalysts (see "Hard-Matter Challenges" section) in which the catalytic center is bound within or on the surface of a hard matter structure. Many of the principles and challenges for all of these catalysts are strikingly similar, however it is most convenient to discuss particular catalyst classes within the context of the soft or hard environments that surround them.

For homogeneous (and heterogeneous) catalysts, we desire the capability to create and utilize metal-organic (or metal-free) ligand arrays capable of achieving heretofore unknown or currently inefficient transformations. Catalytic processes to achieve thermodynamically unfavorable transformations ("going up hill") offer a problem of great significance as exemplified by the challenge to achieve selective and efficient transformations of inert feedstocks such as $\mathrm{H}_{2} \mathrm{O}, \mathrm{CO}, \mathrm{CO}_{2}$, or $\mathrm{CH}_{4}$. Examples would include not only efficient water-splitting to $\mathrm{O}_{2}$ and $\mathrm{H}_{2}$, but also $\mathrm{CO}$ or $\mathrm{CO}_{2}$ (a greenhouse gas) conversion to more benign materials, or methane (another

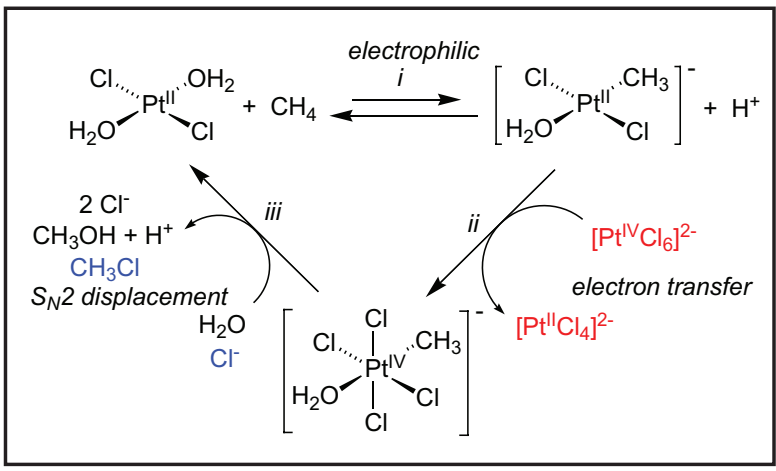

FIGURE 3.4. Homogeneous catalytic cycle for the selective conversion of methane to methanol in aqueous solution using well-defined platinum catalysts via a recently elucidated pathway. Compare to the biocatalyst of Figure 3.5. greenhouse gas) dehydrogenation (e.g., to the useful polymer building block ethylene and $\mathrm{H}_{2}$ ). Such reactions could be driven by coupling to thermodynamically "downhill" catalytic processes or by using radiation fields in the presence of catalysts to drive them. The solar radiation falling on the earth's surface delivers enormous amounts of untapped energy.

In the same vein, the current production of silicon (for metallurgy, computer chips, and solar cells) from abundant $\mathrm{SiO}_{2}$ and of elemental phosphorus (for biological building blocks) from commonly available phosphorus oxides is enormously energy-intensive. In the presence of appropriate catalysts, which currently do not exist, it might be possible with the appropriate coupled reactions or sunlight to transform abundant starting materials to more valuable and/or environmentally benign substances. To address these important problems will require far greater sophistication in catalyst design and assembly than is currently possible.

Another great challenge in catalysis is to selectively transform simple saturated hydrocarbons into alcohols, olefins, and other useful soft-matter substances under mild conditions with high selectivity. Nature achieves highly selective hydrocarbon functionalization processes

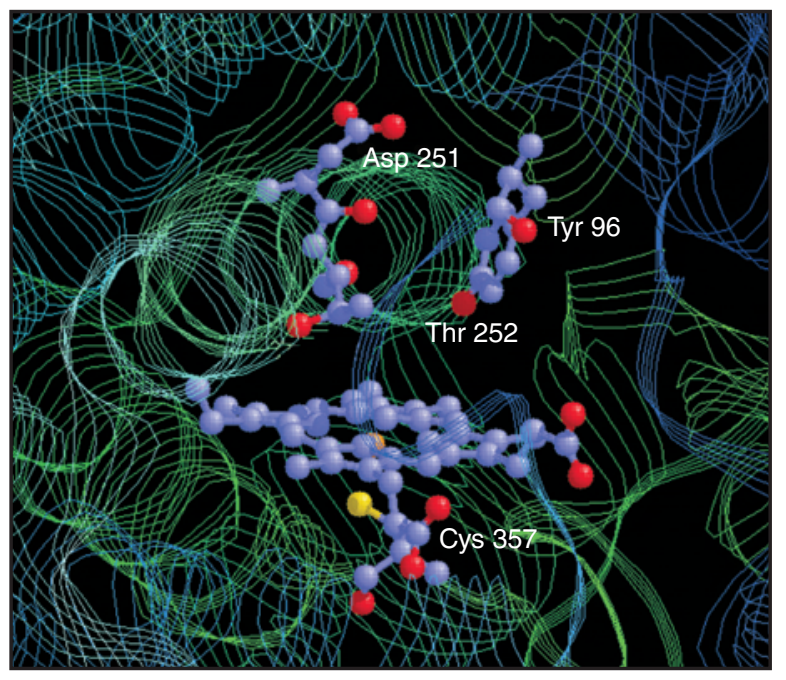

FIGURE 3.5. Active site of a heme mono-oxygenase enzyme $\mathrm{P}-450_{\mathrm{CAM}}$ that nature uses to oxidize small molecules such as hydrocarbons with $\mathrm{O}_{2}$ and selectively produce alcohols. Here the catalytic properties of the central iron atom (orange) are tuned by the surrounding planar porphyrin ligand and the exquisitely "engineered" protein environment superstructure. Compare to the simple platinum catalyst of Figure 3.4. 
in aqueous solution; however, such conversions can be limited in scope, sluggish, and very enzyme- and substrate specific (compare Figures 3.4 and 3.5). We desire far greater understanding of how to achieve such transformations and to exceed Nature's capabilities using synthetic catalysts.

We also desire the knowledge to selectively create conjugated carbon-rich material structures, such as $\mathrm{Cn}$ polyhedra, nanotubes, graphenes, diamonds, etc., at low temperatures and with high structural selectivity. Many interesting and important carbon-rich materials (e.g., C60 "Buckyballs", single-wall carbon nanotubes, graphene sheets, etc.) are currently prepared catalytically at very high temperatures by processes offering little selectivity in "dialing-in" the desired product or placing the product at a specific location on a substrate. New catalysts and catalytic processes could, in principal, offer more selective and hence efficient ways to create these very interesting substances, to modify their electronic and chemical properties in a controlled fashion (e.g., substitute carbon atoms at specific sites with other atoms), and to place them on desired substrates in desired orientations.

Catalytic transformations that selectively create products that consist of a single enantiomer (pairs of enantiomeric molecules are non-superimposable mirror images and are called chiral molecules) with the exclusion of the other are called asymmetric catalytic transformations. Achieving $>99 \%$ selectivity to a single enantiomer (called enantiomeric excess) in any asymmetric catalytic reaction with any substrate capable of producing numerous classes of bio-active agents and pharmaceutical building blocks represents an enormous scientific challenge. Ideally such catalysts could self-adjust to optimize their structures for the required catalytic conversion. Efficient catalytic transformations in which metal-ligand arrays having tailored chiral symmetry create chiral products from achiral reagents can achieve vast multiplications of their own chiralities, hence greatly enhanced atom and energy efficiency over conventional synthetic procedures. We seek new chemistries and levels of understanding to make a dent in such a daunting problem.

In addition to creating small-molecule products, catalysts can also be used to create polymeric materials ("plastics"-materials consisting of long connected chains of constituent building blocks called monomers). Copolymerizing polar (e.g., containing oxygen and nitrogen groups) and non-polar unsaturated monomers (such as ethylene and propylene) with high throughput could produce unique types of polymeric materials. This is an up-to-now "Impossible Dream" because polar substances typically "poison" conventional polymerization catalysts. Achieving this goal would advance our basic understanding of the mechanisms of catalytic polymerization processes, and they would also create new generations of polymers with greater compatibility with polar materials and coatings. Likewise, catalytically creating polymer chains with precisely tailored comonomer or branch incorporation points could afford unique mechanical and processing characteristics. Developing new ways to control polymer-chain sequences would allow tailoring of materials properties to a degree never before possible. A related challenge would be to create copolymers combining both natural and manmade monomers within the macromolecular chain. Efficiently incorporating biological units into abiotic macromolecules or vice versa might create polymers with unique processing, solubility, biorecognition, biocompatibility, and perhaps pharmacological properties.

Another important homogeneous catalytic goal is to dissolve and transform or functionalize previously intractable substances, such as minerals, radioactive wastes, metals, cellulose, or wood, using reagents with superstructures and chemical recognition properties tailored to the particular surface of interest. Many substances with which we desire to perform catalytic or other chemical transformations are intractable for reasons having to do with solubility and/or in the case of wood-like materials, because nature designed the material to resist parasites. New surface-active substances promoting dissolution, de-agglomeration, suspension/ and/or dispersion, or penetration of insoluble matrices for catalytic conversions would be extremely valuable-for example, in permitting direct conversion of biomass into liquid fuels.

\section{Soft-Matter Electronic Properties}

Here we need to know how to maintain the desirable soft-matter properties of mechanical flexibility, processability, environmental compatibility, and light weight 
while introducing hard-matter electronic properties or enhancing distinctive soft-matter properties. Particularly desirable would be to selectively create soft materials with high charge-carrier mobility for either holes or electrons and with controllable carrier densities. Soft matter characteristically has far lower carrier mobilities than hard matter for reasons reflecting differences in long-range order, bandwidths, electron-phonon coupling, etc. Although advances are being made, we currently have neither rigorous theoretical nor workable chemical synthetic design principles that would allow tailoring soft matter to maximize carrier mobility and to select between holes or electrons. To achieve this would have a significant impact on soft-matter applicability in electronics, displays, lighting systems, and solar-energy applications. Related goals would be to realize soft materials with tailorable band gaps, optical cross-sections, luminescence efficiencies, and singlettriplet crossing rates. Here we need to understand better how to tailor both electronic structure and photophysical dynamics. The attraction here is to control photophysical properties at will; ultimately this may be applicable to more energy-efficient small-molecule and polymer displays, lighting systems, and solarenergy systems (see Figure 3.2). For a discussion of how molecular photophysical properties might ultimately be used for computation, see the Sidebar "Molecular Logic."

Successful strategies to manipulate excitonic states (excited states of bound electron-hole pairs) in soft materials would greatly benefit organic solar cells and lighting systems. Creating long-lived excited electronic states with tunable emissive characteristics presents a scientific challenge in synthetic tailoring of soft-matter photophysics that we are barely beginning to understand. Likewise, soft-matter structures that enhance the mobility of tightly bound excitons or their splitting into current-carrying holes and electrons would be highly desirable. If we could master these materials properties, we could create far more spectrally tunable and energy-efficient display materials (see Figure 3.6) and perhaps soft-matter solar cells with unprecedented efficiencies.

Tuning magnetic properties in soft matter presents yet another major challenge. The target here would be

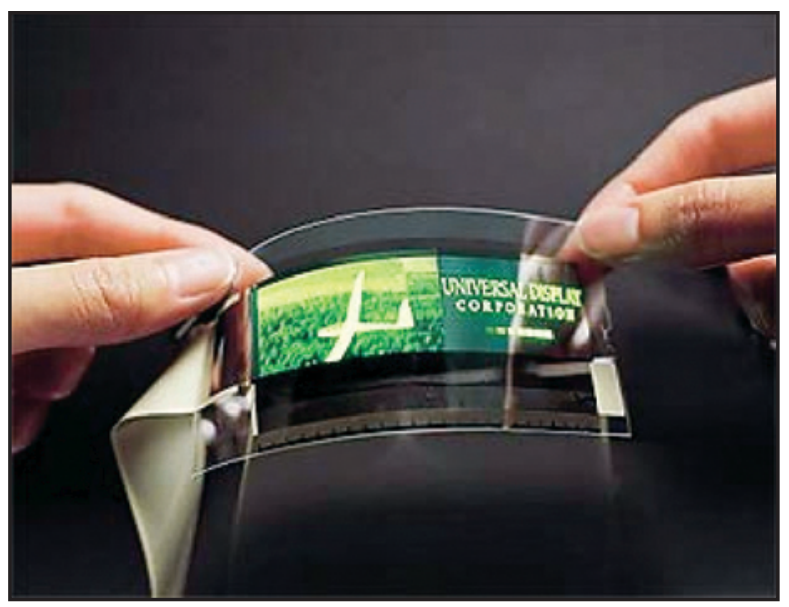

FIGURE 3.6. A prototype OLED (organic light-emitting diode) utilizes flexible organic materials for hole and electron transport and recombination to achieve efficient light emission. Advances in materials design and understanding should lead to more energy-efficient displays and solid-state lighting.

high-performance magnetic materials that might be stretched, molded, or cast like a plastic. Currently, we understand very little about the theoretical limits of soft-matter magnetic properties. much less how we would synthesize them. The same is true of tunable soft-matter optical properties (refractive index, dielectric constant, polarizability, and second- or third-order non-linear optical response). The delocalized $\pi$-electron systems of soft matter offer, in principle, completely unique approaches to creating new types of optical materials, materials for high-speed telecommunications, and materials for energy-efficient high-speed computation using light rather than electrons. To achieve these goals will require far better understanding of how light interacts with delocalized soft-matter electron systems and new synthetic capabilities to realize theoretical predictions of optimum structures.

\section{Soft-Material Mechanical Properties}

Here we are challenged to develop the new knowledge to prepare and understand soft matter that has light weight and great mechanical strength and durability, yet can be formed into shapes or has mechanical properties that can be "switched" on or off in response to a stimulus. Intriguing possibilities include ultrahigh-strength materials that can be processed into films, fibers, and other shaped objects. Traditionally, 


\section{MOLECULAR LOGIC}

\section{Nanoscale Communication Between Molecules Could Lead to Advances in Computation and Communication}

Solar-energy conversion by photosynthetic organisms is based on the intermolecular transfer of excitation energy and electrons. These same processes may be viewed as the nanoscale exchange of information between molecules, raising the possibility that the study of such fundamental phenomena could lead not only to better methods for energy production, but also to advances in computing and communication. Most technological data transmission and processing is based on binary operations carried out by transistors and other devices arranged on the planar substrates of silicon "chips."

This approach has powered the computer age, but as the search for faster, smaller, and cheaper devices continues, several problems loom on the horizon. Smaller switching devices and interconnects between them promise faster computing, but current fabrication technologies are approaching their lower limit for feature size, as discussed in Chapters 2 and 5. In addition, supercomputers now require tremendous amounts of energy. Inefficiencies in current devices contribute to this requirement, generating so much waste heat that further miniaturization of components and concurrent increases in processor capabilities are severely limited.

These considerations are spurring basic research into alternative approaches to data manipulation. The human brain, for example, is capable of prodigious feats, but is made up of organic molecules and systems that communicate via rather slow ionic currents and diffusion of neurotransmitters. Even primitive organisms have large numbers of feedback systems for control of biological function that involve communication between molecular species.

Some researchers are now beginning to explore information exchange between organic or bio-molecules as a prelude to molecular computing (see also Chapter 5). Many of these studies have used chemical agents to activate molecular switches, a process that occurs in the brain. Another approach that offers potential advantages is to use light as the switching agent. Light requires no wires or access for addition of chemical reagents, leads to no buildup of byproducts, and can be delivered on extremely fast time scales. It also allows three-dimensional architectures, which can lead to very high densities of information storage and processing. Light-operated logic devices may also be rapidly reconfigured for different purposes simply by changing the nature and order of the photonic inputs.

The structure in Figure 1 is an example of a lightcontrolled molecular logic gate. By using light of different wavelengths, the molecule may be interconverted among four distinct structures. The porphyrin senses the isomeric composition of the two photochromes via electron-transfer phenomena and reports on the overall structure through modulation of its fluorescence properties. This molecule may be configured by light inputs as a photonic AND Boolean logic gate, an INHIBIT gate, a 2:1 digital signal multiplexer, or a 1:2 demultiplexer.

Molecular logic devices may find application to data manipulation and storage, not as direct replacements for current technology but rather in ways we have yet to imagine.

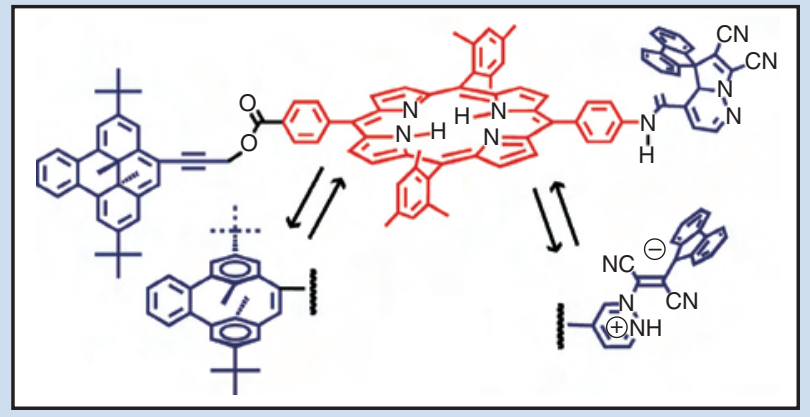

FIGURE 1. Light-controlled molecular logic gate, consisting of a porphyrin (red) flanked by two different photochromic molecules (blue), each of which can be isomerized (arrows) by light between two metastable forms. 
high-strength organic materials have been insoluble and made ductile only with difficultly (see Figure 3.1). We need to know how to manipulate intra- and interchain forces to overcome these deficiencies. In the same vein, mechanical properties that were tunable with a stimulus (thermal, magnetic, radiation, chemical) such as stiff $\leftrightarrow$ ductile, would produce unique materials. Here we desire the ability to "switch" mechanical properties on and off in response to an external activating process to allow molding, extrusion, film blowing, etc., to be followed by "locking-in" of high-modulus characteristics.

Crosslinking (creating links between different polymer chains in a material) that is reversible with a thermal, magnetic, radiation, or chemical stimulus is one strategy for creating "switchable" materials properties. If we knew how to do it, this process could also be used to make stable blends of dissimilar materials as in polymer solar cells or biomaterials for prosthetics. In the same vein, the flow and mechanical properties of many macromolecular materials are strongly influenced by entanglement processes among polymer chains. A significant challenge would be to create entanglement that is reversible with a stimulus (thermal, magnetic, radiation, chemical). We desire here the ability to turn such properties "on and off" in a synthetic polymeric material. A related challenge would be to create polymers in which density was controllable with an external stimulus. The ability to imbue soft matter with this property could lead to materials of interest for functions as diverse as dielectrics for organic electronics and efficient thermal insulation. It might also provide true recycling capability for synthetic materials. To achieve these goals will require new synthetic capabilities closely coupled with new measurement and theoretical methodologies.

\section{HARD-MATTER CHALLENGES}

Hard-matter compounds encompass the great bulk of the periodic table, and only a very small fraction of all the possible compounds has yet been prepared. The traditional thermal approaches to synthesizing hardmatter compounds ("heat and beat") are frequently limited by kinetic and diffusion factors in bringing the reactants together. However, emerging synthetic methods like those of soft matter ("chimie douce"soft chemistry) promise to revolutionize the field. Chimie-douce synthetic strategies may be especially useful for modifying electronic structure by doping, preparing new metastable phases, and preparing materials with high surface areas. Application of these and related strategies could lead to completely new ways of realizing solid matter. One technique, biotemplating, is used by nature to make unique hybrid materials that go beyond the simple soft/hard dichotomy by incorporating both molecular and mineral substructures, resulting in such elegant structures as seashells and bones (Figure 3.7). Other synthetic approaches include using fluxes, reactive multilayers, metal-organic precursors, intercalation/exfoliation, self-assembly, nanoparticle prercursors, high-pressure and high-temperature synthesis, chemical or physical vapor deposition, sol-gel techniques, electrochemistry, and other synthetic techniques. Many of these are newly developed or have not been broadly implemented. New methodologies are also being developed to grow larger, more perfect crystals for measurements and applications. In the future, exploratory synthetic high-throughput experimentation techniques that rapidly generate large libraries of materials will greatly enhance our ability to discover

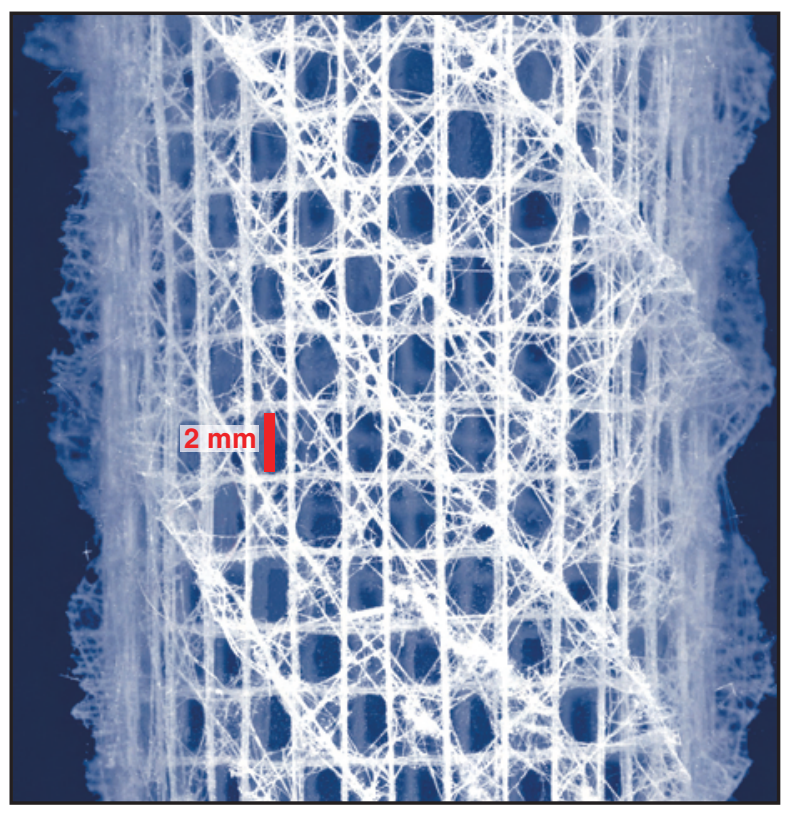

FIGURE 3.7. Example of a natural sea sponge skeleton produced by soft-matter protein templating of hard-matter growth in an aqueous marine environment under mild conditions. The scale bar is $2 \mathrm{~mm}$. 


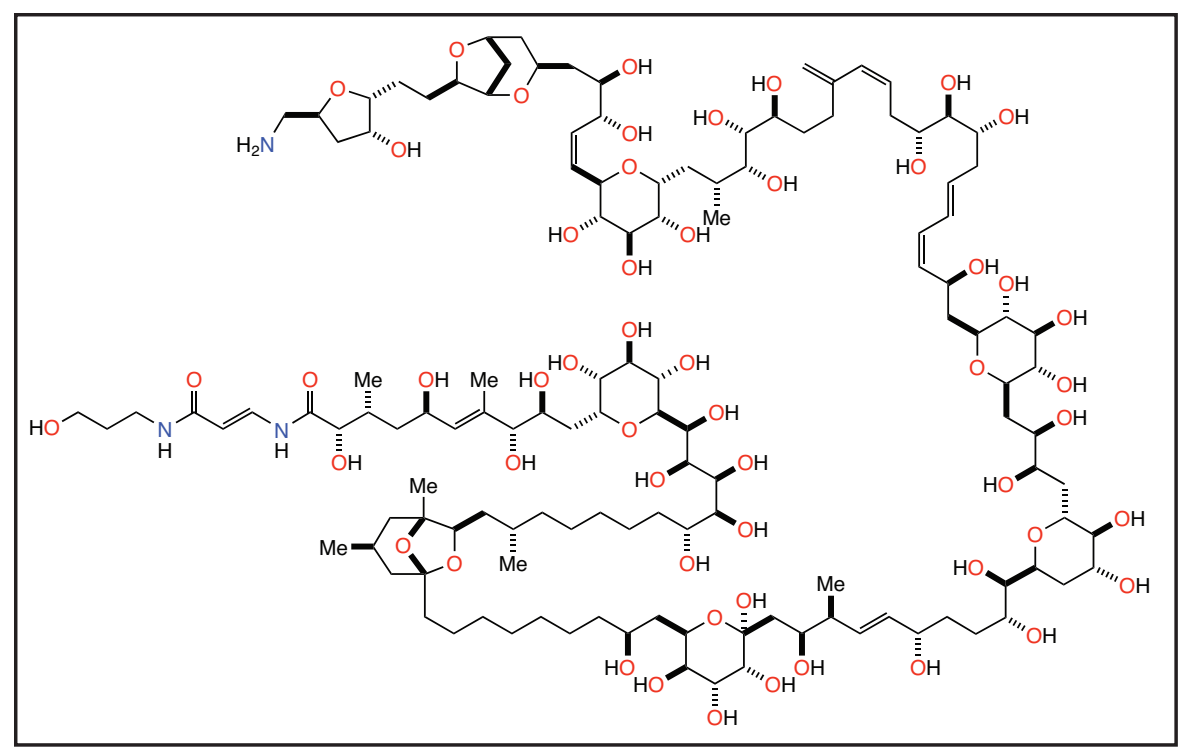

FIGURE 3.8. Palytoxin, a quintessential example of multi-step, soft-matter, fragment-by-fragment, linear chemical synthesis. Can we apply approaches of similar intricacy and elegance to create new forms of hard matter?

new substances when coupled with rapid screening of properties. The products could range from the new highly correlated materials of Chapter 4 to metals and alloys for more efficient battery and fuel cell electrodes to ultra-hard/ultra-strong materials for tools, machines, and aircraft structures, to substances the properties of which lie far beyond our imagination.

What then are the overall challenges in creating hard matter? From the standpoint of new materials creation, can we learn to rationally construct hard matter over diverse length scales with the atom-level "ball and stick" precision and versatility with which we can now engineer soft-matter architectures (Figure 3.8)? Can we precisely tailor crystal lattices over a broad range of length scales (nano- to macro-scale) and dimensionalities for diverse atom combinations? At best, today we have only an inkling of how we might achieve this, and newly developed synthetic methodologies would have to be closely coupled with rapid-feedback, time-efficient, insitu characterization techniques to guide such syntheses and with high-level theory to not only aid in selecting targets but also in selecting promising reactions and reaction coordinates. This is very different from conventional materials creation research and will require new types of interactions and focusing capabilities.

In regard to hard-matter properties, while one doesn't generally confront the fragility typical of traditional soft matter (see the "Soft-Matter Challenges" section above), we find ourselves envying the light weight, ductility, corrosion resistance, steric tunability, and architectural diversity of soft matter. As with soft matter, we are also challenged in the case of hard matter with understanding how mechanically strong we can make a material and yet minimize the weight. How durable can we make it? How resistant to corrosion, decay, or failure in extreme conditions of temperature, radiation, environmental exposure, etc.? How small can a particle of a given substance be and still retain the properties of large pieces of the same composition or structure? If the properties change when the particles become small, how will they change? How do defects control crystallinity and glassiness? Can we learn from (and then surpass!) nature how to make hard matter that "heals" damage or defects? That constantly modifies itself as the surrounding environment changes? Can we learn to stabilize such important non-equilibrium substances as glasses and heterostructures (see Chapter 6)? Finally and operationally most important, what new techniques and strategies will be needed to arrange atoms into such remarkable new substances? These are truly daunting problems and will require new research modalities to address such problems!

One striking yet poorly understood characteristic of hard-matter substances concerns the unique properties of their surfaces. Cyclic sequences of chemical transformations that occur on these surfaces form the basis for heterogeneous catalysis, processes that are capable of effecting extraordinarily diverse and deep-seated chemical 


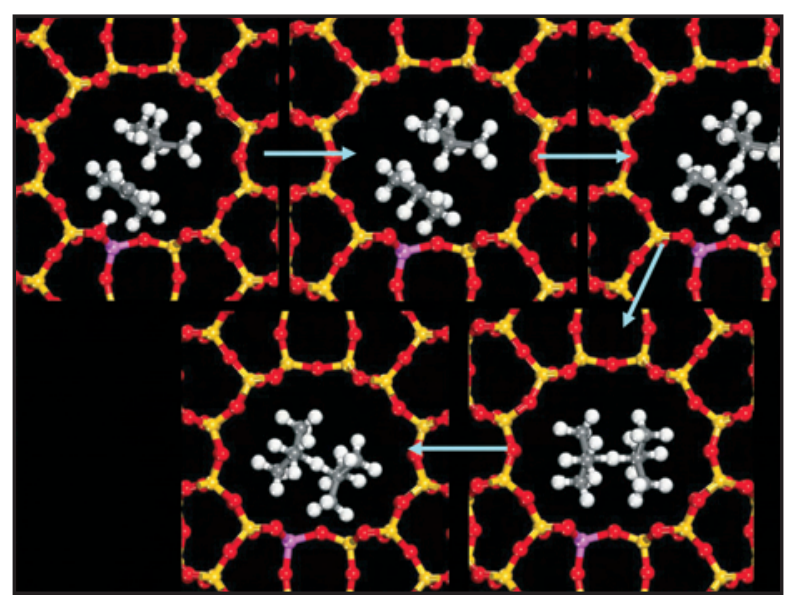

FIGURE 3.9. Computed mechanism for the selective catalytic coupling of two four-carbon hydrocarbon molecules, butane and iso-butene, to form the eight-carbon motor fuel iso-octane in a human-made mesoporous heterogeneous catalyst having acid sites within nanometer-scale channels. Such analyses promise to help us design and realize more energy- and resource-efficient catalysts and catalytic processes.

transformations of numerous organic and inorganic substrates (Figure 3.9). Heterogeneous catalytic processes account for a significant fraction of the U.S. GPD, and new ways to improve them would have enormous scientific and technological value. Their strengths and weaknesses frequently complement those of their homogeneous catalytic analogues discussed earlier - they are far more robust, yet accurately defining their structures and mechanisms has proven far more elusive. Many may depend for their function on stabilized non-equilibrium arrangements of their atoms.

To achieve full mastery and predictive power over heterogeneous catalysts will require a far greater level of understanding than we have today. We require deeper insight into the structures of the actual catalytic sites and the source(s) of their uniqueness, better ability to distinguish "spectators" from "players," knowledge of what percentage of the available sites are active and why, a better understanding of poisoning processes, and a far deeper grasp of the intricate kinetic factors governing catalytic selectivity. To achieve this understanding will require the application of incisive new in-situ physical-characterization tools as denoted in Chapters 2 and 7 that push spatial and temporal resolutions to the limit, along with new theoretical approaches to deal with complex reaction networks far from equilibrium and involving a great many atoms such as discussed in Chapter 6. Equally intriguing is the challenge of combining heterogeneous and heterogeneous catalysts to achieve the best properties of each- "single-site" heterogeneous catalysts. Today we have at best only an inkling of how to prepare them.

Below we discuss some hard-matter scientific challenges. Considering the vastness of hard-matter phenomenology, the focus is necessarily selective. Note that many hard-matter "wishes" closely map on those expressed above for soft matter: the knowledge base to imbue each in a controlled fashion with the most desirable characteristics of the other. For these reasons, we focus on properties not specifically discussed for soft matter and more unique to hard matter.

\section{Contra-Indicated Properties}

Here we seek to understand and master properties that are not normally expected to be present in the same material, yet occasionally are. What is the interplay between these properties and how can their complementarity be manipulated? Transparent conductors represent a challenging example of contra-indicated properties (Figure 3.10). We seek to understand and achieve, in terms of crystal structure, composition and

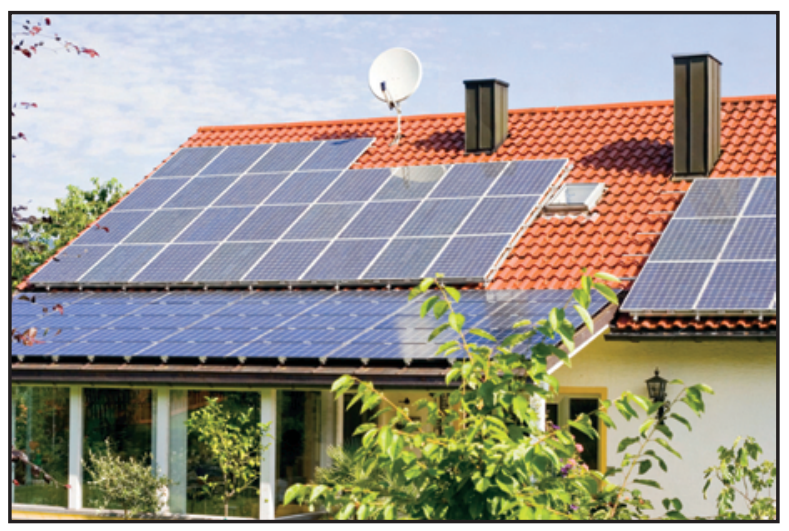

FIGURE 3.10. High-efficiency solar cells use transparent conducting oxide (TCO) electrodes for current collection. Cheaper, more conductive, more corrosion-resistant, and processible materials are needed for more efficient and cost-effective solar cells. 
doping, and processing, the limits to carrier mobility in degenerately doped wide-gap semiconductors, how to maximize the band gap (and optical transparency) and how to produce p-type as well as the more common $\mathrm{n}$-type materials (for $\mathrm{p}-\mathrm{n}$ junctions). We seek to understand whether high conductivities can be achieved with environmentally more benign metal constituents than employed today, and how to deposit such materials on soft-matter substrates (e.g., plastics). Such research could transform the energy landscape by providing far more efficient solar cells, more energy-efficient "smart windows," more efficient sold-state lighting, and completely new types of transparent electronic circuitry.

Thermoelectric materials generate electricity when their junctions are placed in a temperature gradient. Here we wish understand and develop crystal-chemistry strategies at multiple length scales and temperatures to maximize electricity generation and thermoelectric power while minimizing thermal conductivity. What are the realistic limits that can be achieved and how can they be realized? How can we identify ideal laboratory-scale materials and then produce them on a large scale? The ultimate benefit from such advances would be energy-efficiency by turning waste or solar heat directly into electricity and/or by more efficiently air conditioning large structures.

High-temperature superconductors are highly correlated materials in which electrical resistance to current flow completely vanishes at critical temperatures $\left(T_{c}\right)$ sometimes more than $100^{\circ} \mathrm{K}$ above those seen in conventional superconductors. A major challenge is to achieve materials with room-temperature $T_{c}$ values. As also emphasized in Chapter 4, to realize this high-risk breakthrough, we need to fundamentally understand the limits of $T_{c}$ and critical-current density in such strongly correlated systems. We then must learn how to incorporate the accrued crystal chemical and electronic structure knowledge to create high- $\mathrm{T}_{\mathrm{c}}$ hard matter that is environmentally stable and can be readily processed on a large scale into inexpensive flexible tapes and wires. We need to understand and manipulate grain boundaries within high- $T_{c}$ materials as well as their interfaces with non-superconducting materials. This understanding could ultimately lead to new, more sensitive, and energy-efficient magnetic resonance imaging (MRI) techniques, to large-scale levitation processes (Figure 3.11), and to new technologies for high-speed, energyefficient computation.

\section{New Materials for Heterogeneous Catalysis}

As noted above, heterogeneous catalysts embody many scientific challenges in understanding complex sequences of chemical transformations taking place on hard-matter surfaces. Heterogeneous catalysts also play a pivotal role in areas as diverse as the production of transportation and heating fuels, commodity, agricultural, polymeric, pharmaceutical, and fine chemicals, as well as treatment of automotive and industrial exhausts, and their properties nicely complement those of homogeneous man-made and biological catalysts. Deeper fundamental understanding of all catalytic processes as well as insights into creating more active, selective, and robust catalysts holds great promise for cleaner and more efficient energy utilization. Major challenges in heterogeneous catalysis are to more clearly define the nature of the active sites, to engineer at the molecular level catalysis with designed properties in three dimensions, and to create new catalysts for new transformations. Major advances here will require active interplay among instrumentation specialists, synthetic chemists, and electronic structure theorists. Some intriguing opportunities are given below.

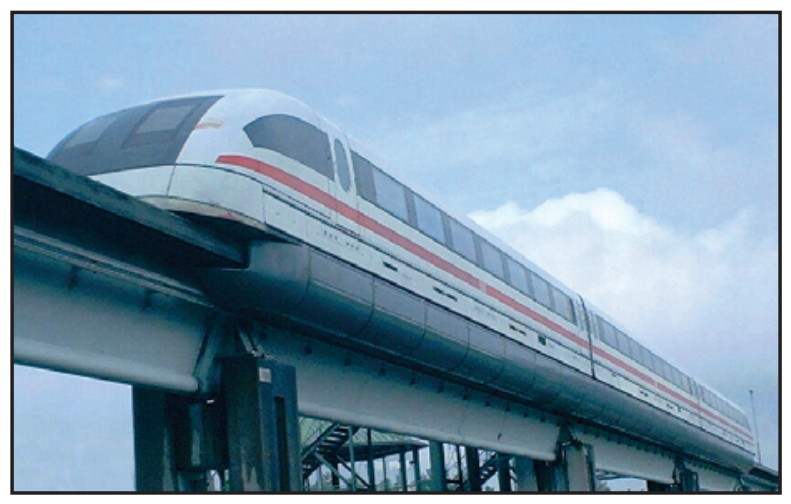

FIGURE 3.11. Magnetic levitation of high-speed trains using low-temperature superconductors. Creating new high-temperature superconducting materials with current-carrying and processing characteristics far superior to those achievable today would provide the means for a complete sea change in transportation systems and in energy transmission. 
Observing catalytic events at the atomic level in real time would greatly advance our understanding of all catalytic processes, and it would move catalytic science toward fulfilling the quest of creating the ultimate catalysts in terms of activity, selectivity, cost-effectiveness, and durability by design rather than by empiricism. The means to acquire incisive "movies" of both homogeneous and heterogeneous catalytic transformations over broad length and time scales is truly a major challenge and depends on successfully addressing the scientific challenges spelled out in Chapter 2. For heterogeneous catalysts, this could completely revolutionize the field. Short of this, but still important, are techniques that can distinguish active catalytic sites from "spectator" or "dormant" sites and to learn exactly how they differ in structure, location, or state of activation.

At the interface of homogeneous and heterogeneous catalysis are "single-site" heterogeneous catalysts. Here we seek to implement the approaches of soft-matter synthesis to create molecular-scale catalyst precursors that adsorb on hard-matter surfaces to create unique catalytic environments. These could include those with chiral-ligand arrays for asymmetric catalysis (see definitions above), with photoactive complexes to achieve challenging "up-hill" transformations such as solarpowered water-splitting into $\mathrm{O}_{2}$ and $\mathrm{H}_{2}$, which would effect the selective polymerization of challenging monomers and would poise dissimilar catalytic centers in proximity, so that unresponsive substrates would undergo enzyme-like bifunctional activation by two or more catalytic centers acting in cooperation. Single-site heterogeneous catalysts might also be used to efficiently carry out cascades of coupled catalytic transformations to achieve sequential stepwise conversions in a single reaction vessel. Generalizable ways to achieve this goal could result in great energy efficiencies. For further insight into catalytic fuel-cell energy-conversion mechanisms from a hard-matter versus biological standpoint, see the Sidebar "Proticity and Electricity" in Chapter 5.

Another major catalytic challenge is to create unique surfaces ("supports") and catalytic environments with three-dimensional aspects. Creating hard-matter surroundings of active catalytic centers that enhance reactivity or selectivity offers great opportunities. These could include extremely electron-deficient or electronrich surfaces that would activate catalytic sites for greater

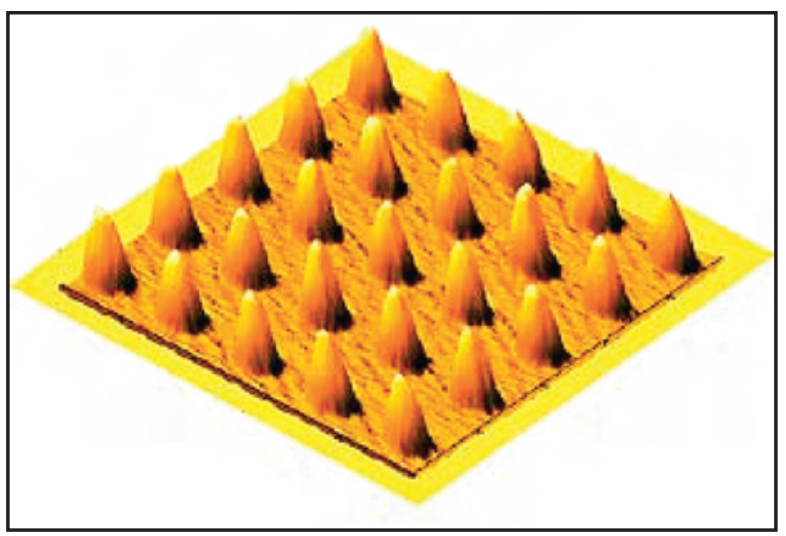

FIGURE 3.12. Regular arrays of 20-nm-high platinum nanoparticles fabricated by electron-beam lithography (a semiconductor-processing technique) on a $\mathrm{Si} / \mathrm{SiO}_{\mathrm{x}}$ wafer for heterogeneous catalytic studies.

electrophilicity (electron-deficiency) or nucleophilicity (electron-richness), or that might serve as "antennae" to concentrate light for more efficient photo-assisted catalysis (e.g., water splitting to $\mathrm{H}_{2}+\mathrm{O}_{2}$ ). In addition, known structures having regular porous structures with Ångstrom or nanometer dimensions could be imbued with regions tuned for enzyme-like hydrophilic or hydrophobic regions; those that would attract substrates, stabilize transition states, and expel products or poisons would be very powerful. Even more challenging would be supports with flexible appendages to introduce enzyme-like "induced fit" dynamic properties, such as opening a passage to the active site for substrate-active site binding, closing to promote reaction (stabilize the transition state), then opening again to expel product. New nanometer-scale lithographic techniques also offer intriguing opportunities to create unique catalytic assemblies placed on substrates with predetermined catalyst coverages and site-site distances (Figure 3.12). For a discussion of hard-matter versus biological approaches to harvesting the energy of sunlight, see the Sidebar "Solar Fuels."

\section{NEW INTEGRATED SYNTHETIC AND ASSEMBLY APPROACHES}

\section{Small Length Scales: Nanostructures}

The science of creating and manipulating materials at very small length scales presents numerous scientific 


\section{SOLAR FUELS}

\section{Nature Provides a Blueprint to Scientists for Capturing, Converting, and Storing Solar Light in the Form of Fuels}

More than 3 billion years ago, nature developed the machinery to capture, convert, and store (CCS) solar energy. One way for us to achieve CCS is to borrow the design of nature in the photosynthetic process. The primary steps of photosynthesis involve the conversion of sunlight into a wireless current. The anodic charge of this wireless current is collected at the oxygen-evolving complex (OEC) to oxidize water to molecular oxygen $\left(\mathrm{O}_{2}\right)$ and the cathodic charge is collected at Photosystem I to reduce the molecule NADP to NADPH, which is nature's form of hydrogen. Thus, the overall primary events of photosynthesis store sunlight by the rearrangement of the chemical bonds of water to form oxygen and hydrogen.

Scientists are now seeking to realize an artificial photosynthetic system by spatially separating solid-state or molecular catalysts for water reduction and oxidation and connecting them to a light-collection and charge-separation system (Figure 1). In one such construct, captured solar light is converted into spatially separated electron-hole pairs within a photovoltaic cell and four of these electron-hole pairs are captured by the catalysts. The solar energy is stored in the bond rearrangement of $\mathrm{H}_{2} \mathrm{O}$ to $\mathrm{H}_{2}$ and $\mathrm{O}_{2}$.

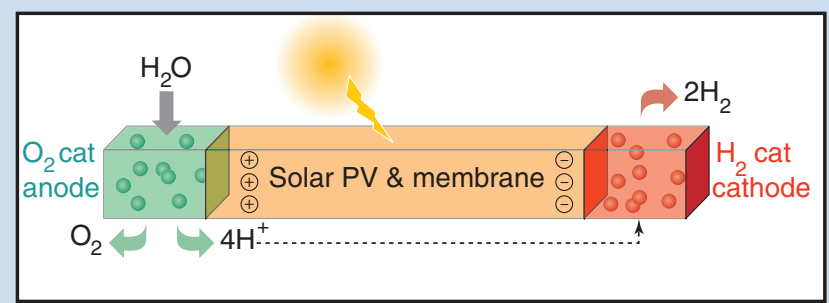

FIGURE 1. Schematic diagram of one concept for an artificial photosynthetic system that begins with spatially separating solidstate or molecular catalysts for water reduction and oxidation.
The blueprint for water splitting has been delivered to science with the appearance of the structure of the photosynthetic membrane (Figure 2). After decades, scientists can now see the biological machine that oxidizes water: $\mathrm{a}\{\mathrm{Mn}\} \mathrm{Mn}_{3} \mathrm{CaO}_{4}$ cluster. Clear principles are revealed by the structure of the photosynthetic membrane: (1) The environment near the water-generating site must be controlled so that two water molecules may be pre-assembled for $\mathrm{O}-\mathrm{O}$ bond coupling; (2) water must be activated by coupling proton-transfer reactions to electron transfer, and (3) high-valent metal, with direct bonding to an $\mathrm{O}$ atom, must be generated for coupling. With these benchmarks, chemists are now working to capture these essential ingredients of the OEC for the assembly of two waters and their activation by coupled proton and electron transfer to produce $\mathrm{O}_{2}$ and protons, which then may be reduced to produce hydrogen.

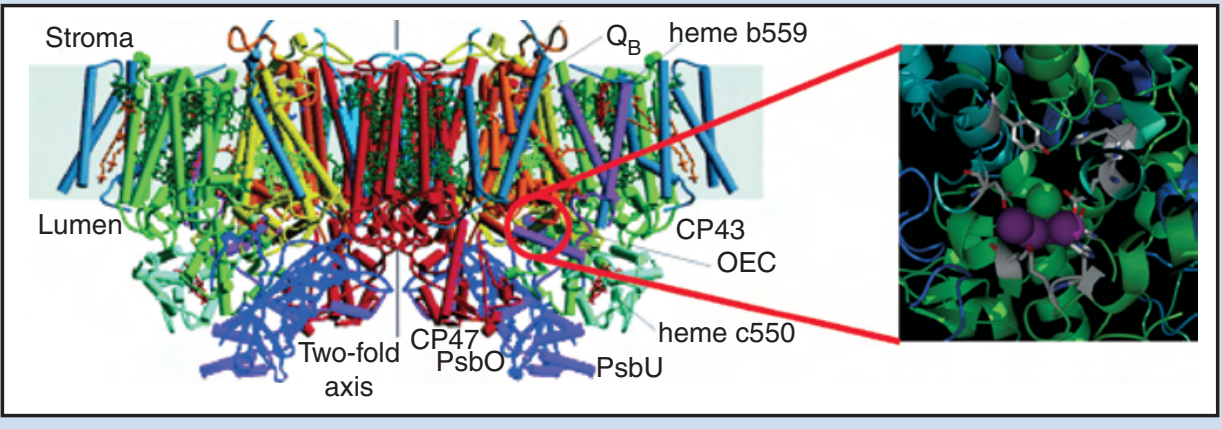

FIGURE 2. Structure of nature's photosynthetic membrane. The inset shows the manganese-based biological machine (OEC or oxygenevolving complex that oxidizes water: a $\{\mathrm{Mn}\} \mathrm{Mn}_{3} \mathrm{CaO}_{4}$ cluster (purple). 
challenges, including how we: (1) synthesize such materials with precise size and compositional selectivity, (2) manipulate their positions at will, (3) scale-up their synthesis and purification, and (4) characterize their properties with depth and accuracy. A key issue central to accounting for every atom in a nanocrystal is achieving completely uniform size in the batches of nanoparticles we synthesize. We seek to understand if it is possible to achieve a true "mole" of nanocrystals, as we can for molecules. Can we know where every atom is, as in a large soft-matter molecule? How many atoms does it take to constitute a nanoparticle "surface"? What tools will be necessary to characterize these surfaces?

Another significant challenge is to devise general approaches to creating alloyed and multi-functional nanocrystals. The principal challenge in accounting for atoms is that at the same length scales (several nanometers) the atoms tend to phase-segregate, and the intended nanostructure is also strained. Can we develop "perfect" alloying in nanocrystals? Can we program into nanoparticles specific kinds of connections or intereactions that lead to the types of "emergent behavior" discussed in Chapter 4? The ultimate products could be new generations of more selective and durable heterogeneous catalysts, components of more energyefficient photovoltaic and display devices, and more sensitive biomedical sensors (Figure 3.13). We also desire to devise ways to assemble nanocrystals into

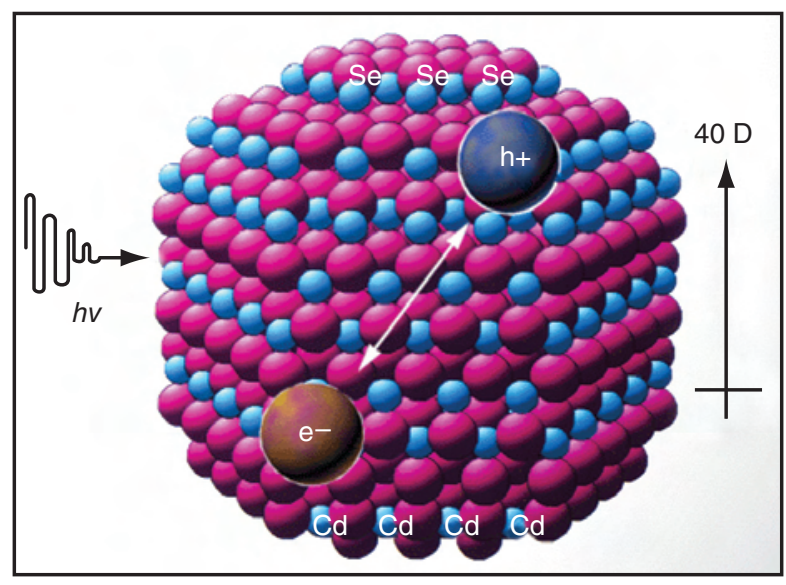

FIGURE 3.13. Photoexcitation of an exciton (an excited state of a bound electron-hole pair) in a semiconducting nanocrystal quantum dot. Such materials and processes can emit light useful for displays, solar energy conversion, and biomedical imaging. regular arrays. Understanding the different forces operative in the assembly of structures with sizes between $1 \mathrm{~nm}$ and $100 \mathrm{~nm}$ and with vastly different physical properties is a new challenge. Can we understand the driving forces and mechanism(s) for assembly? How can external factors (e.g., charge, magnetism) facilitate the assembly of particles into hierarchical structures?

How do we construct phase diagrams for nanomaterials? Nanoparticles are typically referred to as a nonequilibrium "kinetic" or "trapped" state as the result of an arrested growth process. For metal-catalyzed nanostructure syntheses (e.g., carbon nanotubes, semiconducting hard matter nanowires), bulk phase diagrams are traditionally consulted as a first step toward identifying the most appropriate catalyst. Can the equivalent information be obtained for small numbers of atoms? Another major challenge is to monitor nanoparticle chemical reactions in real time. Most nanoscale particles are synthesized under non-equilibrium conditions, and their growth mechanisms are poorly understood. A means to monitor the formation of nanocrystals in real time and with extremely high spatial resolution would afford far greater understanding of the pathways by which they form, hence new ways to manipulate these pathways. Is there a way to observe the nucleation and evolution of very small crystals or determine the growth mechanism at the single-atom level? What time scales are involved? These challenges underlie much of Chapters 2 and 6 .

Nature is rich with nanostructures containing combined hard and soft matter. Indeed, life depends on such hybrid nanostructures as that illustrated by the ferritin protein-iron complex used to store iron as iron oxide nanoparticles in vertebrates, plants, and bacteria. Synthetic hybrid nanostructures include gold quantum dots surrounded by polyatomic ions, first described by Faraday in the 1840s. These are of great interest for ultrasensitive detection in medicine, food, and defense. Related hybrid titania/organic nanodots are the basis of the Grätzel cells used for efficient solar photoconversion. Importantly, design of such hybrid nanostructures is still largely Edisonian, and even the nature of the binding forces remains unclear. Again, both synthetic advances and fundamental theoretical understandings will be required to prepare functional soft matter- hard matter nanostructures by design. 


\section{Realizing Active Nanomaterials Systems}

Here we seek to develop the means to produce large numbers of nanocrystals and to create "engineered" arrays of tailored nanoparticles to execute specific functions. A particularly challenging example would be to design nanoparticle systems using biological and highthroughput experimentation principles. Can the design and evaluation of nanomaterials be based on a systems biology approach or using high-throughput experimentation by which large arrays of experiments are conducted simultaneously under automated control ("combinatorial" experiments) with rapid feedback to efficiently zero-in on optimized materials?

Scaling up the production and purification of nanomaterials also represents an enormous challenge (Figure

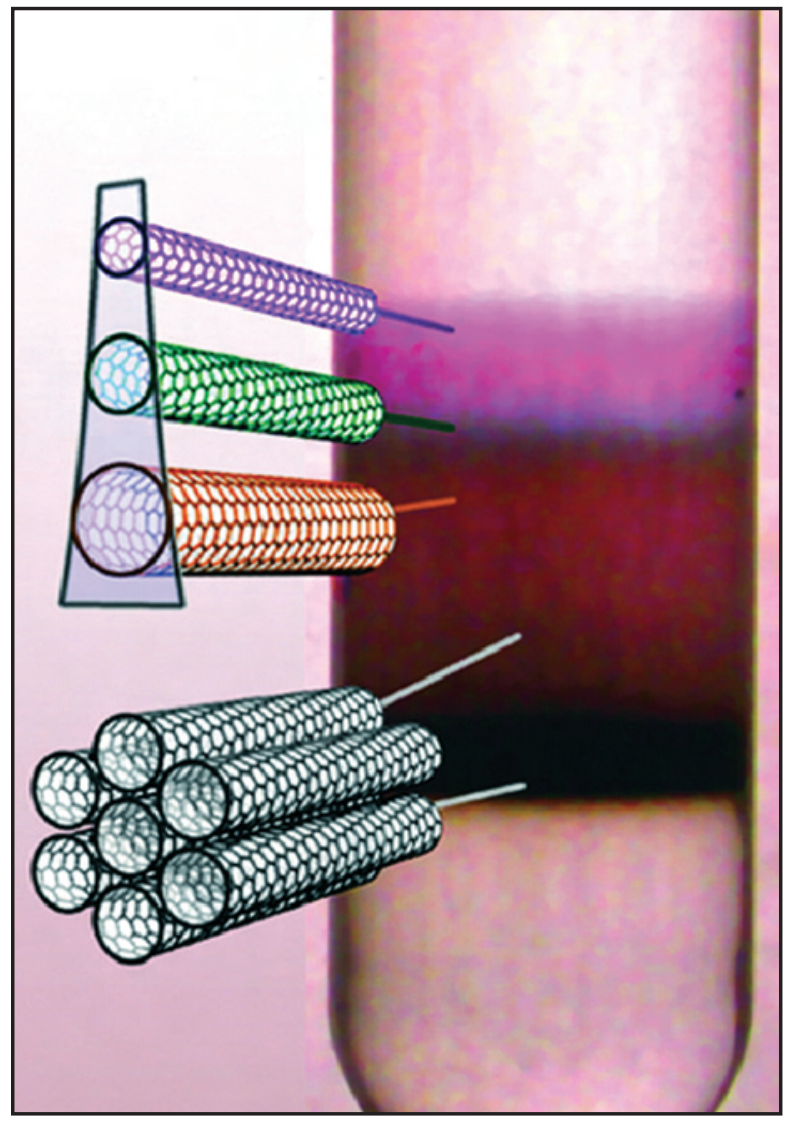

FIGURE 3.14. Centrifuge tube containing single-walled carbon nanotubes (SWNTs) that have been sorted according to physical and electronic structure using density-gradient ultracentrifugation (a biomedical research purification technique). Each colored band contains SWNTs of a different diameter and thus different electronic and optical properties. The sorted SWNTs are then collected for use in a wide range of applications, including electronics, sensors, and displays.
3.14). What new and creative large-scale manufacturing approaches can be devised that can "mass produce" them? It is not obvious that this will be as simple as starting with larger quantities of reagents and larger reaction vessels. New kinds of equipment and reaction procedures are likely to be required if large quantities of these interesting materials are to be prepared.

We also seek to invent new architectures for nanoelectronics and the chemistries and lithographies to create them (Figure 3.15). What innovative ways can be devised to achieve signal transduction? What types of architecture would function optimally?

\section{Achieving Function from Novel Structure: Metamaterials}

Metamaterials are classes of ordered composite structures that exhibit unusual electromagnetic properties not normally found in nature. These properties include new response modalities not observed in the constituent materials that result from artificially structured low-dimensional inhomogeneities (see the Sidebar "Metamaterials").

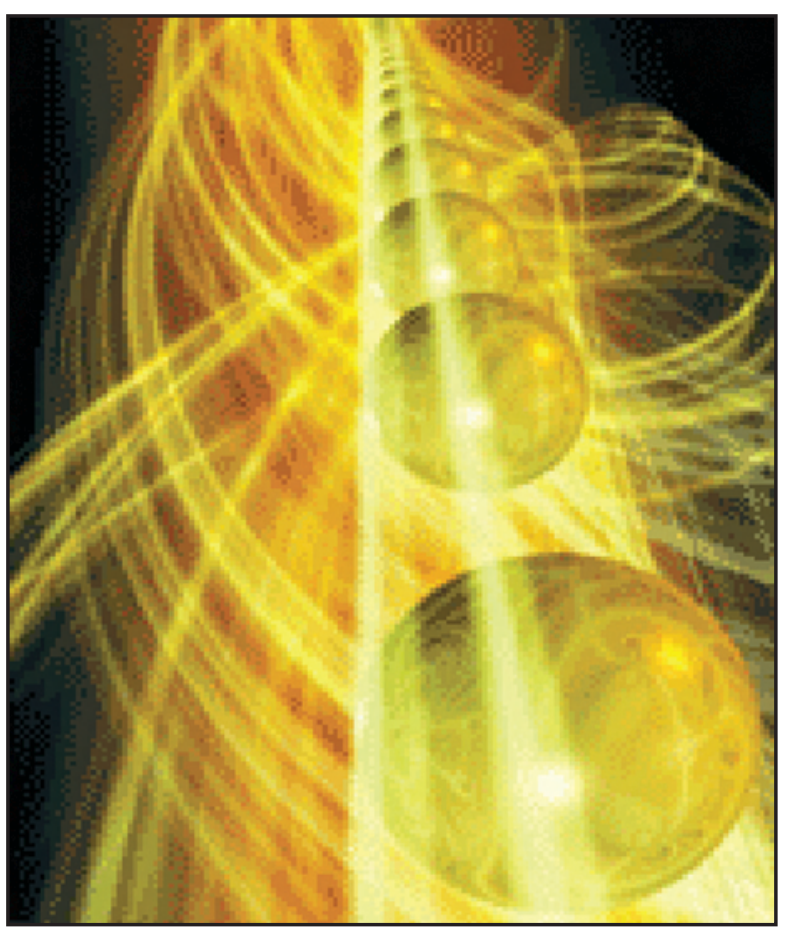

FIGURE 3.15. Representation of the energy transfer that can occur along a one-dimensional array of gold nanostructures. Such phenomena may lead to new information-processing and sensing technologies. 


\section{METAMATERIALS}

\section{Artificially Engineered Structures Have Properties Not Found in Nature and Not Observed in Their Constituent Materials}

Metamaterials have captured the imagination of people from a wide range of backgrounds and ages—-from scientists and engineers who are interested in manipulating light to children and fans of author J.K. Rowling's Harry Potter and his invisibility cloak. Of course the underlying motivation for this interest is quite different. Scientists want to know how structured materials can be designed to exhibit new and exceptional properties; others simply want to know when they will be able to purchase a garment that can render them invisible.

The two most important electromagnetic parameters used to determine how materials interact with light are the electric permittivity $(\varepsilon)$ and the magnetic permeability $(\mu)$, whose values can vary with frequency. Materials that are abundant and naturally occurring (e.g., transparent dielectrics, such as diamond) have a positive $\varepsilon$ and positive $\mu$, and hence a positive index of refraction (n) at optical frequencies. While we experience positive-index materials every day, there are also some natural materials that have a negative $\varepsilon$ and positive $\mu$, such as noble metals (silver, gold, and copper). These materials can be classified as electric metamaterials. In the situation where $\varepsilon$ is positive and $\mu$ is negative, we can speak of magnetic metamaterials, although this combination does not naturally occur at optical frequencies. Finally, when both $\varepsilon$ and $\mu$ are negative, the result is a negative $n$. These materials, also known as "left-handed," do not exist in nature and are referred to as negative-index materials (NIMs).

Metamaterials are constructed from composite (metal and dielectric) building blocks whose size is much smaller than the wavelength of incident light. Thus, their properties can be explained using an effective-medium theory and Maxwell's equations. For example, the first negative electric metamaterial was a periodic array of metal wires described by an effective $\varepsilon$ and a low plasma frequency $\left(\omega_{\mathrm{p}}\right)$ that depended on geometry and not materials. Similarly, a negative magnetic metamaterial could be constructed out of split-ring resonators to exhibit an effective $\mu$ and a high $\omega_{\mathrm{p}}$. A combination of these structures (wires + split rings) resulted in the first NIM, which operated at microwave frequencies (Figure 1).

The idea that materials could be "left-handed" was first introduced in 1968. For instance, left-handed media would follow an inverse Snell's law, where light would be refracted at the interface on the same side to the normal as

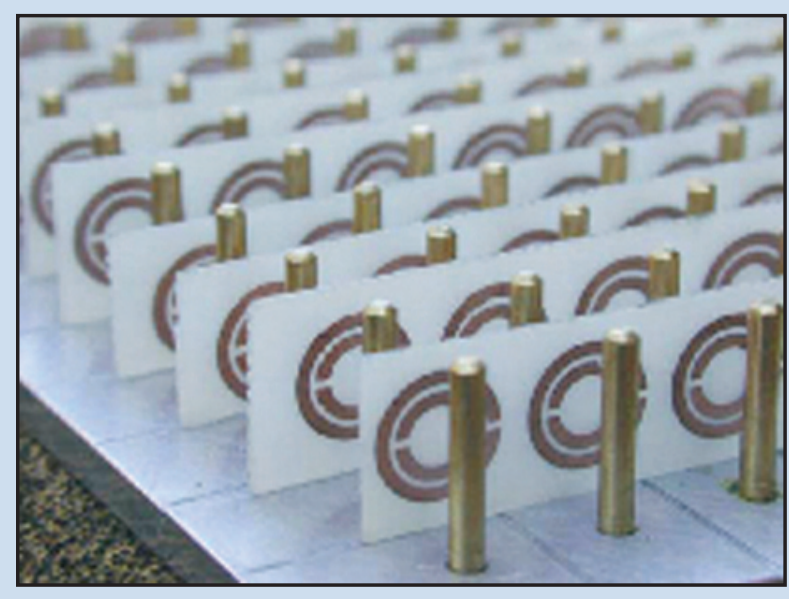

FIGURE 1. Negative-Index Material (NIM) that operates at microwave frequencies. 
the incident light. In the past decade, there have been several demonstrations of negative-index behavior at different wavelength regimes, from the microwave to the optical. All NIMs to date have been created using topdown, fabrication methods. For such structures to realize their full potential, more sophisticated techniques will need to be developed, especially for creating three-dimensional architectures.

Besides the intrinsic scientific interest of these new artificially structured materials, their applications that have attracted extensive recent attention. Two prominent applications are using a NIM as a perfect lens and as a "cloaking" device—and with good reason. What scientist would not want to use a planar or structured metal slab to achieve sub-wavelength imaging with visible light down to $10 \mathrm{~nm}$ ? Or use a NIM to control and bend light around a three-dimensional object at will? Although there have been some initial realizations of these applications, the community still has a long way to go to meet the full potential of these interesting materials (Figure 2).

Metamaterials offer a challenge for designing and controlling the architecture of hard materials. Specific wavelength ranges, in particular, have their own set of challenges. At ultra-low frequencies or DC, can materials be developed for screening sensitive equipment from strong magnetic fields? At microwave frequencies, can electromagnetic waves be controlled using metamaterials? Can low loss materials that are fully three dimensional be produced? At optical frequencies, can subwavelength resolution be achieved exploiting plasmonic structures? Can surface plasmons (collective oscillations of free electrons) be directed and controlled using structured surfaces? Is there a way to layer silver or design low-loss alternatives from new materials to mitigate undesired optical absorption? Also, for optical metamaterials to be more effective, they need to be designed to exhibit an increased magnetic response, which is very weak at optical frequencies.

Negative index materials (NIMs), a type of metamaterial that exhibits a negative permittivity $(\varepsilon)$ and negative permeability $(\mu)$, offer additional challenges. For example, the most common materials for the metallic component of a metamaterial are copper (the microwave region), silver (the visible region), and gold (the near-infrared). Metals are "lossy" because

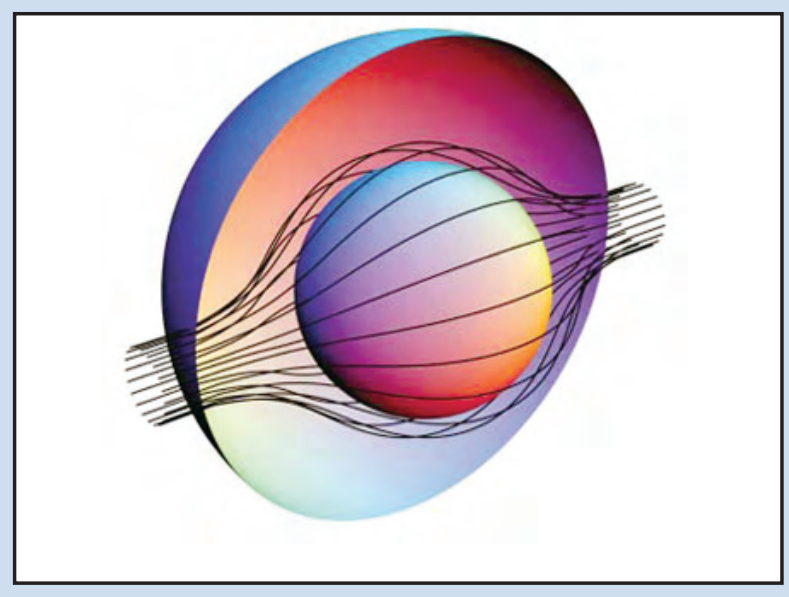

FIGURE 2. Calculated trajectories in three dimensions of $x$ rays in a metamaterial-cloak around a sphere. they absorb light. Are there other materials that can satisfy the requirements for NIM behavior? What would be the materials design rules here?

Metamaterials indeed pose a substantial challenge for designing and controlling the architecture of hard materials. One challenge of NIMs, for any wavelength regime, is to construct them to have a response over a broad range of wavelengths. The two most intriguing applications of NIMs—-subwavelength imaging and electromagnetic cloaking — have only been demonstrated at a single wavelength and in two dimensions (Figure 3.16). For optical metamaterials to be more effective, they must be designed to exhibit an increased magnetic response, which is very weak at optical frequencies, and

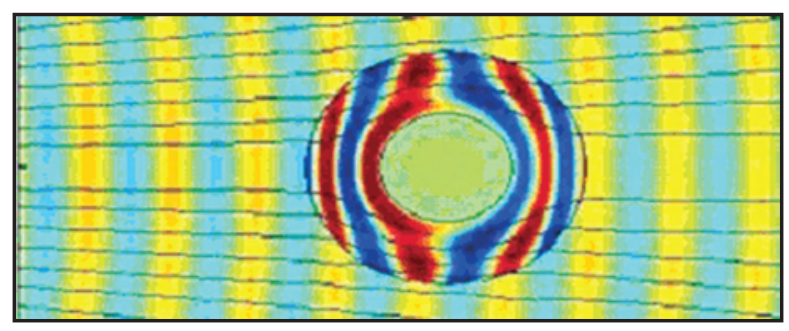

FIGURE 3.16. Electromagnetic cloaking at microwave frequencies using a material with a negative refractive index. Such materials can be used to manipulate light in completely new ways. 
to have reduced loss. Also, an ideal cloak would have minimized absorption and scattering (to achieve transparency), not depend on the object to be hidden, and operate at wavelengths other than the microwave. These challenges may appear daunting, but the scientific journey and the promising applications will certainly be worthwhile.

\section{Discovering New Materials by "Reverse Design"}

An intriguing long-range challenge of broad potential impact at the intersection of synthesis, theory, and characterization and in principle applicable to both soft and hard matter would be to specify a desired electronic structure for a particular desired property of a material, then to iteratively search computationally for the combination of atoms and crystal structure that exhibits this electronic structure (Figure 3.17). Could one then compute the thermodynamic stability of this phase? Would it be stable? What structure would it adopt? What properties would it have? Could one also compute plausible, low-energy synthetic pathways connecting this phase to starting material phases? To achieve this goal will surely require new theoretical approaches (Chapter 4) and new computational facilities (Chapter 7).

\section{CONCLUSIONS}

Understanding, rationally modifying, and improving the architecture and properties of the "stuff" that all things are made of is of central importance to the progress of modern science and to enhancing the quality of human life on earth. This is a common challenge that unites the science of hard and soft matter. Indeed, the boundaries between these traditional categorizations are expected to rapidly fade in the coming decades, and truly Grand Challenge research initiatives should recognize and capitalize on it. Today we have the unprecedented opportunity to conceive, synthesize, and process revolutionary new materials starting from first-principles theoretical design concepts and to characterize them at a spatial, temporal, and energetic depth never before imagined. To address such a truly Grand Challenge will require dedicated long-term research efforts across disciplines, unconventional and highly productive collaborative teams, new kinds of shared facilities, and dedicated pursuit of the goals outlined in Chapters 2 and 4 through 7. In this way, we can truly move beyond the dreams that inspire us to the working techniques by which stuff is made.

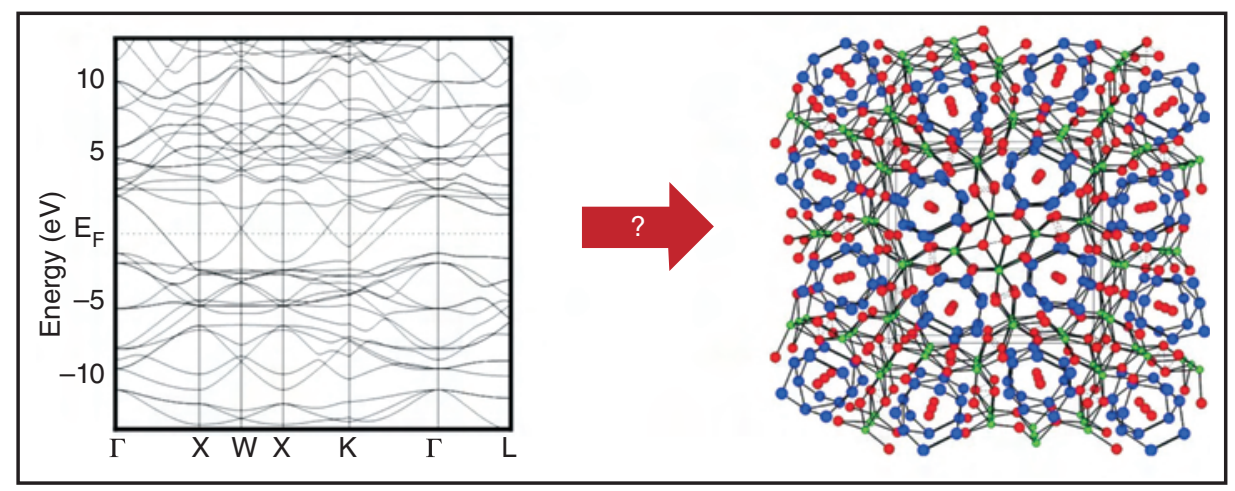

FIGURE 3.17. Materials invention by "reverse design." Is it possible to propose an electronic structure having desired properties and then computationally search for the optimum combination of crystal structure and elements that yields this band structure? Could computation be used to choose the synthetically most promising pathway? 


\section{EMERGENCE OF COLLECTIVE PHENOMENA: STRONGLY CORRELATED MULTIPARTICLE SYSTEMS}

The Grand Challenge for Collective Phenomena is to discover how remarkable properties of matter emerge from complex correlations of the atomic or electronic constituents and to control these properties.

Crystalline diamond is both a precious gemstone and an interesting material with outstanding hardness, excellent heat conductivity, and other remarkable properties. It is also one of the simplest solids known, built from carbon atoms alone. Viewed at the atomic scale, these atoms in their equilibrium state form an intricate lattice with each carbon atom bonded to four equidistant neighbors (Figure 4.1), forming a tetrahedron, one of the five ideal solids envisioned by Plato. The bonds that tie neighboring atoms together in diamond are among the strongest in nature. Because of them, each atom influences and is influenced by its neighbors: we say that they are correlated. The net result of these atomic correlations is the existence of the crystal and its remarkable properties. For example, the hardness of diamond results from both the strength of individual bonds and the perfection of the correlations, or alternatively, the rarity of defects in the crystal. In a one-carat

(0.2-g) diamond without defects, one can follow a chain of 10 million of these minute tetrahedra from one side of the crystal to the other with such precision that the relative locations of carbon atoms at either end of this chain remain fixed to within a fraction of the size of a single atom.

Such "emergent" phenomena, in which the correlated behavior of many particles (in this example, atoms) leads to an unexpected collective outcome (a crystal), are of great significance across the sciences and engineering. In the basic energy sciences that are the subject of this report, the particles may be microscopic like electrons, atoms, or molecules, or may be larger entities such as sand grains, cells in an organism, or rocks in the earth's crust.

The concept of emergence is rather general and permeates all fields of scientific endeavor. One can readily find emergence in many examples throughout this report, including climate change, earthquakes, cell colonies, neurobiology, plasma physics, and life itself. In neurobiology for example, each neuron is a single relatively well-understood cell. Yet billions of these neurons produce consciousness. How and why? In
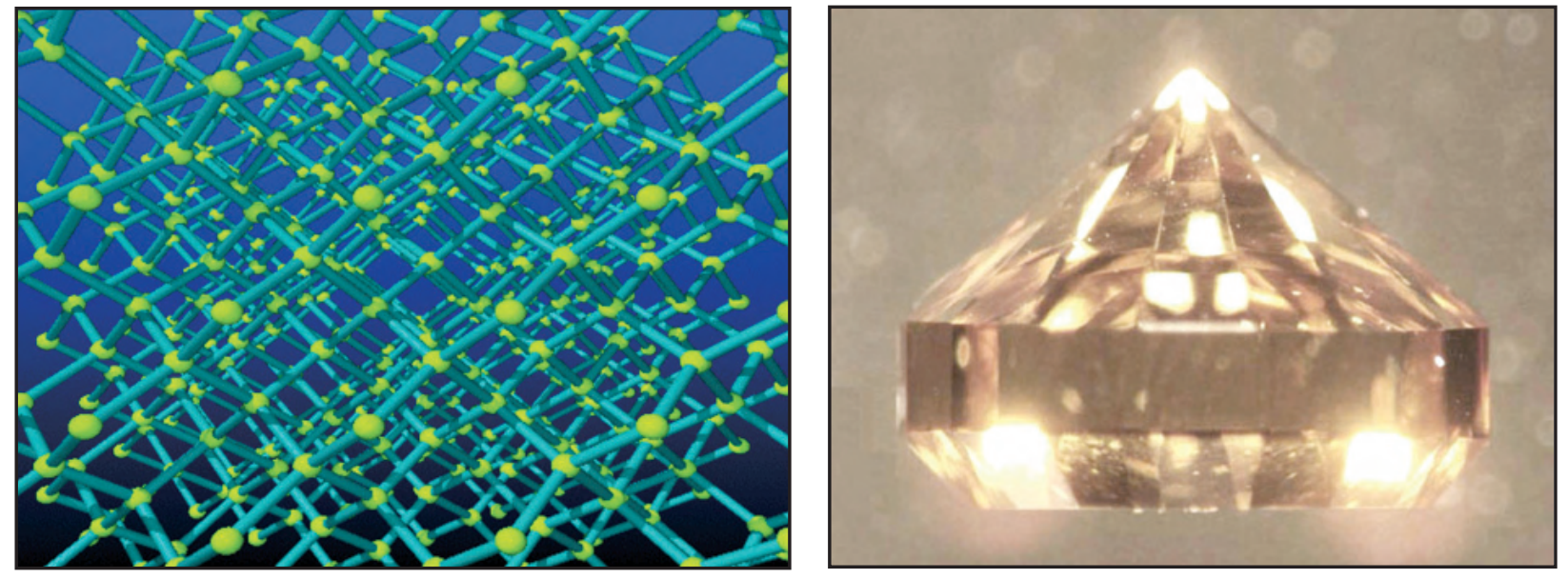

FIGURE 4.1. Left: The diamond lattice. Each carbon atom (yellow sphere) is bonded to four neighbors in a tetrahedral arrangement. The correlations in the atomic positions in the lattice persist over macroscopic length scales. Right: An artificial gem-quality diamond made by deposition of carbon vapor. Many of the extraordinary macroscopic properties of this diamond, such as its clarity and hardness, result from the perfect correlations of the atoms in the lattice. 
addressing such questions, scientists endeavor to find general answers that apply to broad classes of materials, organisms, or phenomena. For instance, the theory of symmetry breaking at phase transitions describes aspects of the solidification of crystals, of the onset of magnetism and superconductivity, and even the formation of the early universe. Uncovering the fundamental rules of correlations and emergence is the first part of the Grand Challenge of this chapter. These fundamental rules are also connected to the concepts of coherent control (Chapter 2), of the fundamental composition of matter (Chapter 3), of nanoscale communications (Chapter 5), and of matter far from equilibrium (Chapter 6).

The second part of the Grand Challenge is to achieve control over those correlations, a prospect that can only now be reasonably contemplated with the advent of tools to probe and affect particles and their correlations on the nanoscale (see Chapter 7). By understanding and controlling correlations, we can put emergence to work for us. The potential applications are as rich as the variety of emergent phenomena. Given this, we cannot hope to be encyclopedic. Our aim is instead to inform the reader of the importance of this type of scientific research, identify some of the other Grand Challenges to which it is connected, and discuss what is needed to make progress in this vital area.

\section{CORRELATIONS AND EMERGENCE: AN OVERVIEW}

A fundamental type of emergence involves correlations between electrons in crystalline materials, which induce a staggering variety of novel phenomena. Research on such materials occupies a large fraction of the physics, materials, and chemistry communities and will be the major focus of this chapter. Electrons are responsible for nearly all electrical and magnetic properties of solids. For instance, magnetism, which arises in part from the intrinsic spin of an individual electron (which can be visualized as a tiny bar magnet), only occurs through strong electron correlations, which align the spin axes of different electrons relative to one another. Magnetism in solids takes diverse forms. In ferromagnetism, known to the ancient Greeks, the spins are all parallel, and a macroscopic magnetic moment with the familiar properties of an ordinary bar magnet results. Beginning in the $20^{\text {th }}$ century, an everincreasing variety of more complex types of magnetism have been discovered, often denoted inappropriately by the single category of antiferromagnetism. These have diverse applications, such as in non-volatile information storage and reading in information technologies.

Another famous example of electronic emergence is superconductivity, in which the electrical resistance of a material vanishes, accompanied by a variety of bizarre macroscopic manifestations of quantum mechanics. Many more types of electronic correlations are under active study. Each distinct phenomenon of this type could provide new functionality for future technologies. For instance, multiferroic materials, which combine ferroelectricity (the emergence of a permanent electric polarization) and magnetism, could enable devices that couple electric and magnetic action (see Figure 4.2).

To realize the potential of such correlated-electron materials, we must control these correlations. Inspiration for the level of control that might be achieved comes from present-day semiconductors, which underlie the phenomenal success of modern electronics. Conventional semiconductors, and their successors that are only dreams today, will continue to advance our technologies and our society, from how we process information and communicate, to harvesting solar energy with efficient photovoltaic solar cells and lighting our homes and workplaces. Under ordinary conditions, however, semiconductors are weakly correlated electron materials, and this characteristic limits their functionality in key ways. A major challenge is to achieve precise processing and control for intrinsically strongly correlated materialswhere these correlations are pervasive, even under ordinary conditions - similar to what is routinely achieved for semiconductors today; this flexibility together with the wealth of emergent phenomena of strongly correlated materials would open up remarkable possibilities. Correlated materials could be greatly superior to conventional materials for problems ranging from efficient charge injection in photovoltaic devices to lossless electrical transmission over superconducting lines. 


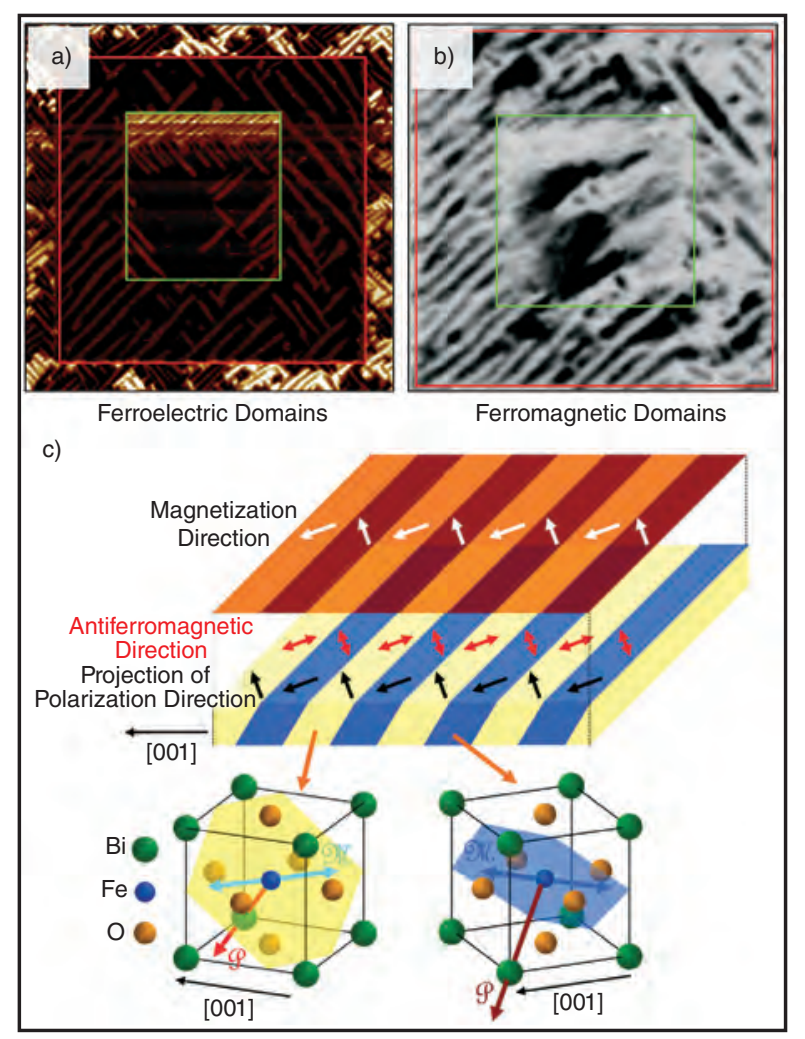

FIGURE 4.2. Two-stage electrical control of magnetism using multiferroics. (a) Piezoforce microscopy image reveals the ability to control ferroelectricity with an electric field in the model multiferroic $\mathrm{BiFeO}_{3}$, which in turn changes the antiferromagnetic structure. (b) Photoemission electron microscopy image showing that the exchange coupling between a ferromagnet ( $\mathrm{CoFe}$ ) and the antiferromagnetism of the multiferroic controls the magnetic structure in the ferromagnetic layer. (c) A schematic that illustrates the nanoscale coupling between the multiferroic and the ferromagnet. Such a multiferroicbased device could provide an efficient link between conventional electronic logic and spin-based logic (see Sidebar "Entanglement in Chapter 2), which offers the potential for significantly reduced energy consumption compared to present-day integrated circuits.

Moving up the scale of lengths from electrons, we encounter correlations of atoms or molecules. The example of diamond discussed above is just one of a cornucopia of beautiful crystals that are formed by atomic correlations. Production of high-quality crystals is critical to the study of the electronic correlations just described, and has much broader applications through the optical, mechanical, or thermal properties of these materials. Synthesis of high-quality single crystals and thin films of highly correlated materials has lagged dramatically in the United States and will require renewed investments particularly with the vast reduction of research activities in industry (see Sidebar "Crystal-Growth Facilities"). Other types of atomic and molecular correlations are subtler than crystallinity and can lead to important materials such as amorphous (meaning non-crystalline) solids and glasses. These correlations, which determine the limits of the mechanical strength of such materials, are just beginning to be understood (see Figure 4.3 and Chapter 3). In soft materials, larger constituents can include polymer chains or lipid molecules that self-organize into gels, membranes, or meso-phases. Such self-organization could be used for advanced lithography of patterns that would be laborious or impossible to produce in any traditional way.

Perhaps the ultimate examples of correlations and emergence are the massively interacting networks of structures in biology that give rise to the amazing capabilities of living things. "Particles" from DNA to proteins to cells to entire organisms within an ecosystem produce large-scale behavior in ways that are largely mysterious to us. However, more detailed knowledge of these constituents and their interactions is available to scientists now than ever before, through the tools of modern biology and nanoscience (Chapter 5). Understanding and controlling correlations in biology based on recent advances in understanding of the fundamental constituents is thus another feasible but extremely difficult scientific challenge.

In the next section of this chapter, we discuss some of the remarkable science of correlated materials, as well as the prospects for, and science of, "semiconductor-style” structures built from them, which are only
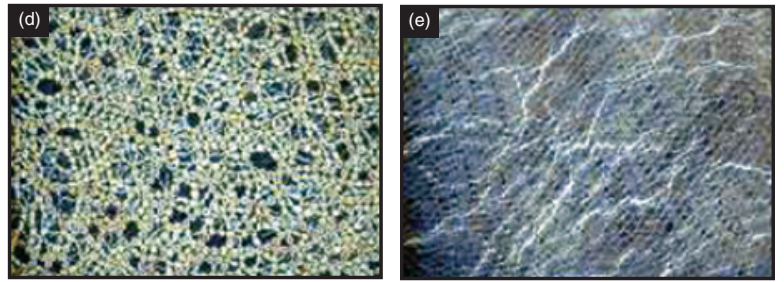

FIGURE 4.3. Images of the stress field (brightness indicates higher stress) in an amorphous "solid" mixture of two sizes of photoelastic polymer disks confined in a single two-dimensional layer. Note the intricate patterns of stress due to the complex spontaneous distribution of contacts, which becomes more filamentary as the mixture approaches the "unjammed" state (right) and the mixture "melts." A theory of these stress correlations, necessary for predictive power, is in its infancy (see also Figure 6.5). 


\section{CRYSTAL-GROWTH FACILITIES}

\section{Functional Crystals Range in Size from 100 Nanometers All the Way up to 1 Meter, and the Facilities for their Growth Are Also Diverse in Size}

Crystalline materials are at the heart of most electronic, optical, and sensing technologies and are also essential for understanding fundamental principles of condensed matter. The size scale at which a material becomes "crystalline" depends on its use. Polycrystalline materials, synthesized to determine the basic electronic or magnetic response, are composed of grains of single crystals typically $100 \mathrm{~nm}$ in size. Large single crystals, such as those used for optical frequency conversion for the large lasers at the National Ignition Facility (Lawrence Livermore National Laboratory) are roughly $1 \mathrm{~m}$ in size.

Not surprisingly, facilities for crystal growth also span a range of sizes. Some crystalline materials form from the melt, much like sugar crystals grown from a solution of water. When the solutions melt at low temperature and are non-toxic, like water, an inexpensive box furnace will suffice for high-quality crystal growth. However, many compounds of interest dissolve only at very high temperatures (greater than $1500 \mathrm{C}$ ) or in toxic fluxes (e.g., lead, bismuth, and hydrochloric acid) and require greater infrastructure investments for safe operation. Certain compounds, usually involving volatile elements, must be grown at high pressure (many kilobars). Finally, other elements require combinations of such growth conditions, thus adding to the complexity of infrastructure.

When crystals are synthesized for industrial applications, such as semiconductor devices or optical components, cost considerations drive the development of large single-crystal boules. Modern apparatuses (see Figure 1) are required not just for the manufacture, but also for R\&D of such materials.

Even for fundamental science, requirements for large crystal size (e.g., for neutron scattering) or extremely high purity (e.g., for novel semiconductor compounds) have driven the development of crystal-growth apparatuses that fall
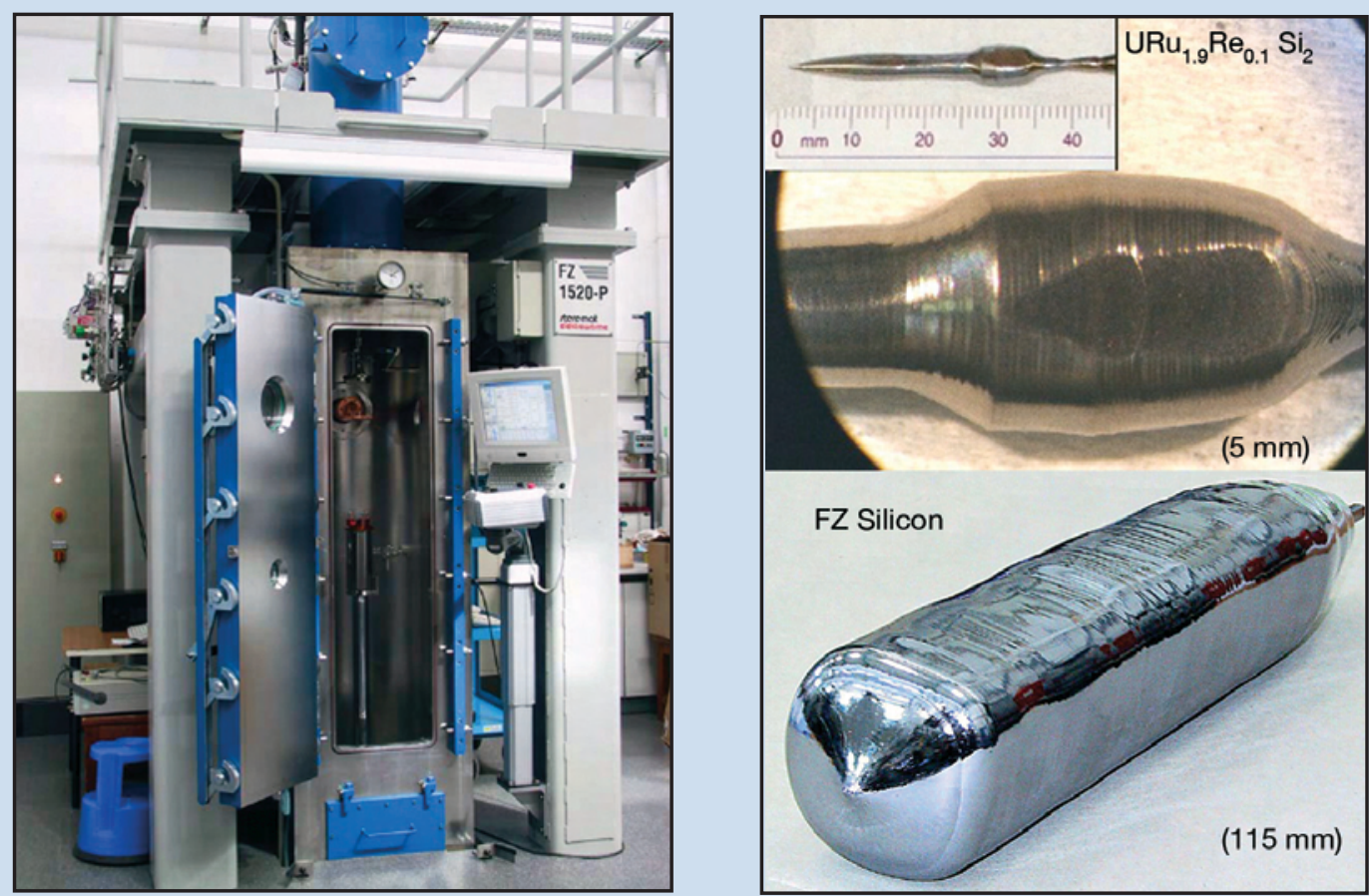

FIGURE 1. Float-zone furnaces are used for the growth of large high-purity crystals. Left: Pressure floating-zone apparatus. Right: Examples of crystals grown in this way: a classic strongly correlated system -a heavy fermion superconductor $\left(\mathrm{UR}_{1.9} \mathrm{Re}_{0.1} \mathrm{Si}_{2}\right)$ lightly doped with rhenium-and silicon. 
into the mid-scale instrumentation category. A prime example is the optical float-zone furnace, which has become the workhorse for growth of large high-purity crystals of oxide compounds. In such an apparatus, the use of optical energy to create a molten zone obviates the crucible, thus releasing limitations on crystal size and purity. Such oxide crystals are required for scanning-probe microscopy, neutron scattering, and angle-resolved photoemission of strongly correlated phenomena that challenge the present understanding of matter.

It is important to emphasize the interplay of different crystal-growth techniques in the maturation of an important material. This can be illustrated no better than in the case of high-temperature superconducting copper oxides. The basic phenomenon of superconductivity in this class of materials was discovered in polycrystalline samples grown in simple furnaces. The phenomenon of high- $T_{c}$ is so exotic and challenging, however, that high-purity crystals, such as those grown in optical float zone furnaces, are required to understand the fundamental mechanisms at play. This example, as well as many other recent examples, shows that to fully promote the discovery and understanding of new compounds for either science or technology, crystal-growth facilities with a wide range of infrastructure size and capability will have continued relevance.

beginning to be achieved in the laboratory. Following this, we turn to examples of strong correlations in other areas of basic energy sciences, and then discuss what is needed for progress in this Grand Challenge area.

\section{CORRELATED ELECTRON MATERIALS AND HETEROSTRUCTURES}

Semiconductors enable the most influential technologies of our time, and semiconductor devices pervade every aspect of life. The spread of cell phones and the Internet throughout society is changing the fundamental nature of human interactions. Within pure science, the impact has been similarly dramatic, with at least four Nobel prizes attributable directly to semiconductor materials, including the 1956 prize for the discovery of the transistor in silicon and the 1998 prize for the fundamental physics of the fractional quantum Hall effect in gallium arsenide. These two discoveries were both enabled by advances in the creation and understanding of heterostructures (structures combining different materials). The trillion-dollar global semiconductor industry that arose from the phenomenal success of the transistor now also promises to address energy and global warming challenges through more costeffective and efficient photovoltaic cells and lighting.

Several factors have contributed to the dramatic success of semiconductors. From a materials point of view, they can be consistently produced with very high quality and fabricated via scalable, high-throughput manufacturing into complex structures. Their key scientific properties are their sensitivity to small perturbations, such as chemical doping and applied electric fields, and the simplicity with which this sensitivity may be described. Semiconductor research is a thriving and important part of the study of hard materials with numerous impacts in basic energy sciences.

Despite all their advantages, there are limitations to current semiconductor materials and devices, and there is no doubt that tantalizing new capabilities might be possible with a departure from this paradigm. Semiconductors are materials with intrinsically weakly correlated electrons: the electrons in the semiconductor act approximately independently of one another. This is a reason for their simplicity, but it is also a limitation. Much richer phenomena can occur in materials in which particles are highly interdependent. In fact, correlation physics is observed in semiconductors under extreme conditions of ultra-high purity, very low electron density, very low temperature, and especially very high magnetic fields. Away from such extremes, however, we suggest that a promising direction for new science and technology that goes beyond conventional semiconductors is to utilize materials that naturally incorporate strong correlations.

Specifically, we envision a major effort to reproduce the amazing progress of semiconductor physics, chemistry, and materials science in new correlated-electron materials, which could create entirely new functionalities as well as higher information densities and processing 
speeds than possible today with conventional materials and approaches. The resources and facilities needed for the success of this effort are discussed at the end of this chapter and in Chapter 7.

\section{What Led to the Success of Semiconductors?}

The combination of factors that led to the remarkable success of semiconductors is formidable to match. The most significant are:

- High-quality materials. The best semiconductor materials, silicon and gallium arsenide, are routinely made with astonishing structural perfection. Even commercial silicon wafers are grown so that the most common impurity, an oxygen atom, occurs only once for every 50,000 silicon atoms. For specialized uses, silicon and other materials (notably gallium arsenide) can be produced in far higher purity. Furthermore, high-quality interfaces between semiconductors and certain other materials play crucial roles in these technologies. For example (as shown by atomic resolution imaging in Figure 7.2), a thin layer of silicon dioxide usually acts as the "gate oxide" in a modern fieldeffect transistor. The interface between gallium arsenide and aluminum gallium arsenide is critical to the creation of nearly ideal two-dimensional electron gases, the basis for many scientific discoveries such as the integer and fractional quantum Hall effects.

- Flexible fabrication. Semiconductors can be manipulated spatially with remarkable precision. They can be grown by successively depositing one atomic layer at a time and with varying composition. They can be patterned within the growth plane by a variety of lithographic techniques with sub-micrometer resolution.

- Simplicity. Semiconductors behave electronically with almost an ideal simplicity. For nearly all purposes, the microscopic complications of the crystal lattice of nuclei and an even higher density of electrons interacting together quantum mechanically can be ignored. Instead, the materials behave like a featureless "vacuum" in which small quantities of particles of negative and positive charges—electrons and holes-behave as they would in free space, provided that one only treats their mass as an effective parameter characteristic of the material and includes a dielectric constant that partially screens their Coulomb interactions. This simplicity makes understanding and modeling semiconductor behavior relatively easy and reliable.

- Sensitivity. Along with the above factors, which highlight the robustness of semiconductors, they also exhibit a complementary sensitivity to small changes of parameters. For instance, the conductivity of a semiconductor can be modified by many orders of magnitude by the introduction of small controlled concentrations of specific impurities (doping), and by the application of electric fields using nearby metallic electrodes (gating). This sensitivity is essential for the usefulness of semiconductors, since it is precisely such modulation of their properties in space and time that allows devices to function. For example, the conductivity of a field-effect transistor is controlled by modifying a voltage on a gate electrode. The science of this sensitivity is a result of specific materials properties characteristic of semiconductors: a large dielectric constant, a small effective carrier mass, and a small band gap.

For another material to supplant semiconductors, even for specialized applications, it must overcome at least one of the limitations of semiconductor materials while remaining cost-effective. What are these limitations? One is a practical limit on the minimum feature size in a semiconductor. As mentioned above, since electrons and holes in a semiconductor behave like particles in free space, the basic electronic unit is just an "effective atom," whose size is simply its effective Bohr radius. Because of the same properties that make their modeling and manipulation simple—small effective carrier mass, large dielectric constant, and small band gap-this Bohr radius is typically large compared to true atomic scales. In silicon and gallium arsenide, for instance, the effective Bohr radius for electrons is approximately 45 and 190 times larger, respectively, than the true Bohr radius of the hydrogen atom. The volume inside this bound state contains many 
thousands of atoms. Thus there is much room for improvement, in principle, by shrinking the size of these effective Bohr radii.

Another limitation is functionality. Because the ground state of a semiconductor is rather featureless, basically the only degrees of freedom available are those of artificially induced (extrinsic) electrons and holes. These are dilute and mainly suitable for controlling the charge density. There is notably no intrinsic magnetism or significant displacement of the atomic lattice. Magnetism (correlation of spins) and ferroelectricity (correlated displacements in the atomic lattice) are key features that enable other types of phenomena and technologies. They are of course used already in a variety of applications, but not with anywhere near the same flexibility as found in semiconductor processing.

\section{What Are the Unique Capabilities of Correlated Materials?}

Strongly correlated electronic materials are promising candidates to provide new functionality, which cannot be found in semiconductors, in part because they exhibit complex types of ordering. Emergent properties of correlated electron materials include ferromagnetism, antiferromagnetism, unconventional and high-temperature superconductivity, charge- and orbital-density waves, electronic phase separation, stripes, ferroelectricity, colossal magneto-resistance and magneto-capacitance. Obtaining even one of these properties in conventional bulk semiconductor materials is difficult, although correlations can become much stronger in semiconductor heterostructures at low temperature. The tendency for ordering even at room temperature in correlated materials results from strong Coulomb interactions between electrons confined tightly in $\mathrm{d}$ or $\mathrm{f}$ orbitals. The small size of these orbitals also means that many phenomena vary over distances much shorter than the large effective Bohr radii encountered in semiconductors, potentially allowing smaller electronic devices in correlated materials.

A second reason to look to correlated materials is their ability to respond sensitively to small perturbations. As discussed above, such sensitivity is critical to the broad utility of semiconductors. In semiconductors, this ultimately results from the small binding energies and large sizes of impurity states (the "effective atoms" that contribute electrons to the device) and their small band gaps. Other weakly correlated materials, which are metals or strong insulators, are much less sensitive, either (as in metals) having so many intrinsic charge carriers that their number can be only negligibly modified, or (as in insulators) having such a large gap and large impurity binding energies that these cannot be controlled. A semiconductor lies between these two extremes and in a sense achieves its exquisite sensitivity because it is close to the transition between metallic and insulating phases.

Strongly correlated materials very often show strong sensitivity to small perturbations for a similar reason: they are "close" to several different types of ordered phases. For instance, in materials known as Mott insulators, Coulomb repulsion forces a fixed number of electrons to partially fill an atomic shell on each atom, leaving undetermined the spin and orbital state of these localized electrons. The secondary effects of kinetic energy, magnetic exchange, and coupling to distortions of the ionic lattice are necessary to pick out the ultimate phase. As a result, there are often several rather different electronic phases that are quite close in energy, and which one is chosen is decided by very small changes in material parameters. This physics is important both for high-temperature superconductors and for certain magnets (see Sidebar "Frustrated Magnetism”). Different electronically ordered states can even coexist in the same material, as for example in materials that combine superconductivity and magnetism, traditionally thought to be mutually exclusive.

\section{Why Are Correlated Materials Challenging?}

Research on correlated materials is not completely new. For instance, the concept of an antiferromagneta material in which electrons are localized on atoms by strong Coulomb repulsion and their spins arrange themselves in a regular but non-uniform pattern-was recognized in the 1930s, and was confirmed experimentally by neutron scattering in 1949. However, the breadth of phenomena in correlated materials and their relative complexity in comparison to semiconductors means that today, both the understanding of strongly 


\section{FRUSTRATED MAGNETISM}

\section{Frustration - the Presence of Competing Interactions that Cannot be Simultaneously Optimized-Leads to Correlated Materials Whose Properties Can Be Controlled by Very Small Chemical Changes}

The spinel structure is one of the most common mineral forms. It is composed of three types of atoms with the generic chemical composition $\mathrm{AB}_{2} \mathrm{X}_{4}$. Usually, the $\mathrm{A}$ and $\mathrm{B}$ atoms are metals, and the $\mathrm{X}$ is a chalcogen (oxygen, sulfur, or selenium). When the structure is magnetic, spins can reside on either or both of the A or B sites. Taken alone, the A sites form the well-known diamond lattice, while the B sites form the so-called pyrochlore lattice (Figure 1).

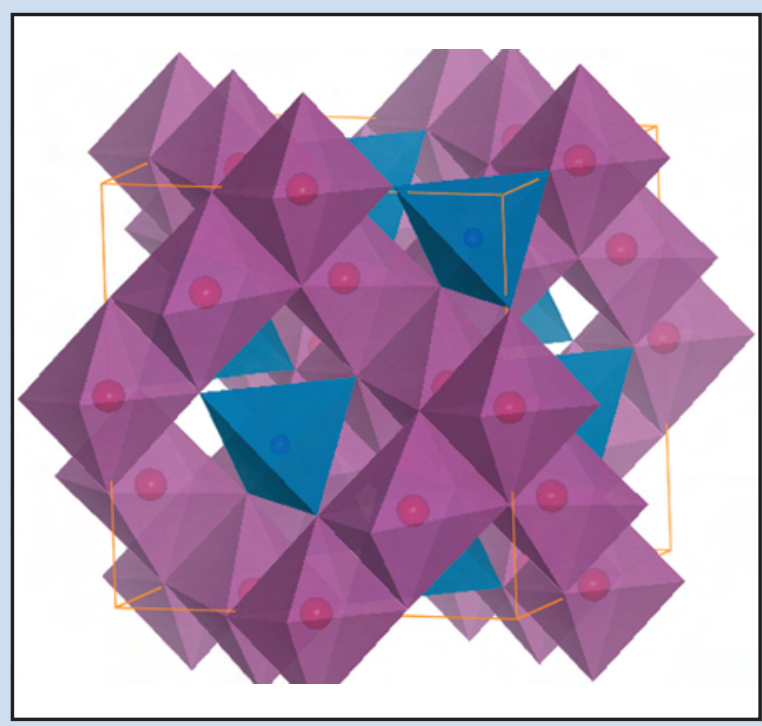

FIGURE 1. Spinel structure. A and B atoms reside inside the tetrahedra and octahedra, respectively.

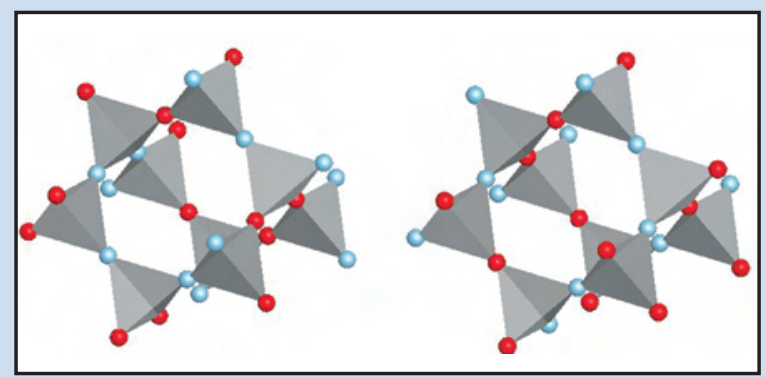

FIGURE 2. Two antiferromagnetic spin structures in $\mathrm{HgCr}_{2} \mathrm{O}_{4}$.
The pyrochlore lattice is a network of corner-sharing tetrahedra. It is one of the most frustrated geometries for antiferromagnetism (neighboring spins are antiparallel) because it contains many triangular faces, and three spins on a triangle cannot all be antiparallel (Figure 2). Spinel magnets illustrate the diversity of behavior of frustrated systems, as well as their sensitivity to small changes in parameters.

Three examples are discussed here; in all cases the B atom is chromium, which in its preferred $\mathrm{Cr}^{3+}$ state creates a frustrated pyrochlore lattice of isotropic spins.

When the $\mathrm{A}$ atom is non-magnetic, $\mathrm{A}=$ zinc $(\mathrm{Zn})$, cadmium $(\mathrm{Cd})$, or mercury $(\mathrm{Hg})$, and the $\mathrm{X}$ atom is as small as possible, $\mathrm{X}=$ oxygen $(\mathrm{O})$, the distance between magnetic $\mathrm{Cr}$ spins is minimized, and their direct exchange coupling dominates their behavior. These materials are beautiful examples of nearly ideal "Heisenberg" pyrochlore antiferromagnets. They exhibit strong frustration, which is relieved by their coupling to the lattice, so that their low-temperature antiferromagnetic ordering is accompanied by strong lattice distortions. The strong correlations in their "spin-liquid" state above the ordering temperature have been observed directly in $\mathrm{CdCr}_{2} \mathrm{O}_{4}$ by neutron scattering (Figure 3). Interestingly, these three materials all order differently, giving further evidence of the sensitivity of strongly correlated materials to perturbations, in this case minute differences in the magnetic and lattice interactions induced by changing the $\mathrm{A}$ atom.

If the non-magnetic $A$ atom is replaced by a magnetic one, e.g., $A=$ cobalt $(\mathrm{Co})$ or iron $(\mathrm{Fe})$, the materials become ferrimagnetic, i.e., show coexisting ferromagnetism and antiferromagnetism. The antiferromagnetism in $\mathrm{CoCr}_{2} \mathrm{O}_{4}$ has a spiral character. This induces an electric polarization, and the two are strongly coupled. Indeed, recent experiments have demonstrated control over the electric polarization by magnetic manipulation of the magnetization. 


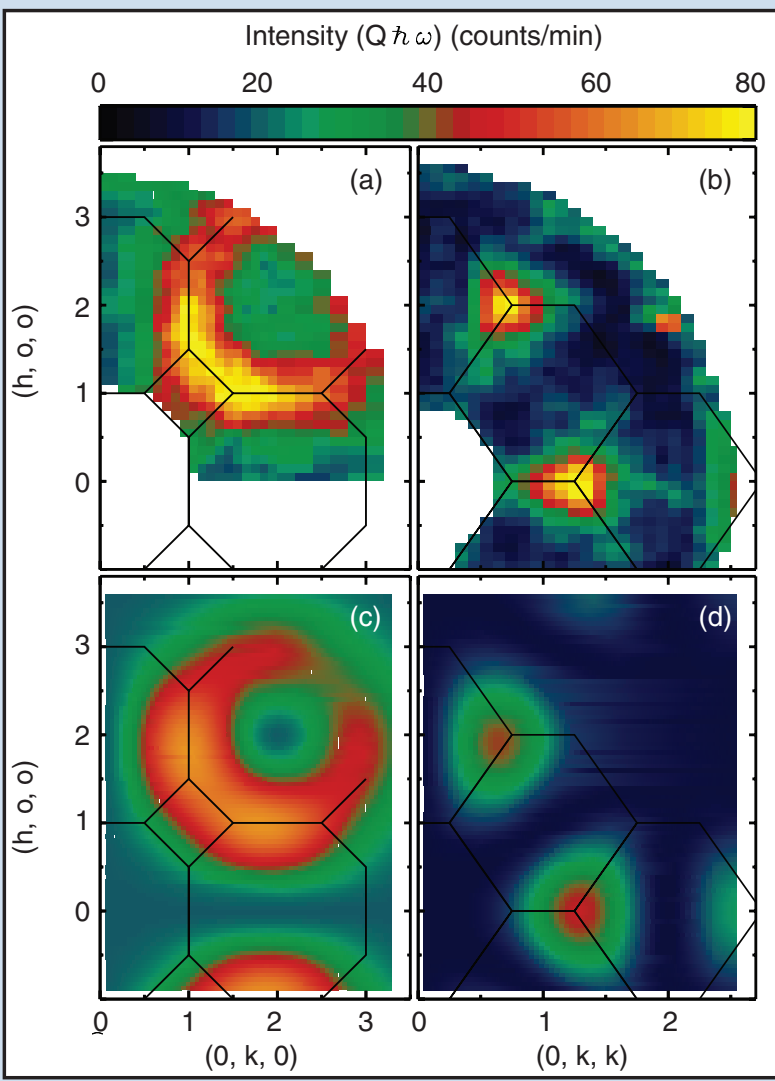

If instead, the A atom remains non-magnetic, but the $\mathrm{X}$ atom is replaced by a larger $\mathrm{S}$ or $\mathrm{Se}$ atom, the lattice is expanded and the distance between $\mathrm{Cr}$ spins increases. In these materials, the magnetic exchange is believed to no longer be dominated by direct coupling between the $\mathrm{Cr}$ spins, and the resulting behavior is much more complex. Just as magnetic frustration can induce lattice distortions, it can also couple to the electrical polarization and dielectric constant. In $\mathrm{CdCr}_{2} \mathrm{~S}_{4}$, a strong spin-lattice coupling gives rise to a "colossal magneto-capacitance," an approximately five-fold increase in the dielectric constant upon application of a relatively small 5-Tesla magnetic field.

FIGURE 3. The intensity of scattered neutrons in $\mathrm{CdCr}_{2} \mathrm{O}_{4}$ (shown by color scale) as a function of momentum. correlated materials and the science to synthesize and process them lag far behind that of semiconductors.

Perhaps the most important contrast between semiconductors and correlated materials is that the basic physics, chemistry, and materials science of the former has been understood for decades. Improvements in semiconductors since that time have been primarily in applications and extensions and in adding fine detail to an already well-established scientific framework. In many strongly correlated materials, simply to understand the basic science at the level of the electron-hole theory of semiconductors remains a challenging puzzle. However, this science is being explored and expanded by the impressive array of new experimental and theoretical tools of modern physics, chemistry, and materials research. Furthermore, driven by industry, the ability to synthesize high-quality semiconductor materials and to process them into functional devices far exceeds our proficiency with highly correlated materials such as the complex oxides described below.

\section{SOME KEY PROBLEMS IN CORRELATED ELECTRON MATERIALS}

\section{High-Temperature Superconductivity}

The family of superconducting cuprate materials, which have fascinated and perplexed the community since their discovery in 1986, provide a paradigmatic example of strongly correlated materials. These materials have increased the highest temperature at which superconductivity occurs in solids more than fivefold, to $138 \mathrm{~K}$ (at atmospheric pressure). High-temperature superconductivity remains one of the defining problems for quantum condensed-matter physics. These materials have catalyzed a rich variety of experimental advances, from crystal and film growth to high-resolution photoemission spectroscopy and diverse scanning-probe microscopies, and they have led to a similar improvement in theoretical techniques. As a result, these materials and their superconductivity are much better 
understood today than a decade ago, and an ever more detailed understanding is emerging year by year. Despite these successes, the key puzzles of the cuprates remain unresolved. When they are resolved, it should be possible to design superconductors that work at higher temperatures, possibly even reaching room temperature. If room-temperature superconductivity is achieved, it will truly and totally transform many technologies from transportation to power generation and distribution to imaging.

The greatest practical and fundamental questions in high-temperature superconductivity are related: How high can one push $\mathrm{T}_{\mathrm{c}}$ (the temperature below which the material is superconducting)? What is the mechanism for the superconductivity? A rational approach to the former requires an answer to the latter, which we still lack. The question of the mechanism is an intriguing one, especially this year, the $50^{\text {th }}$ anniversary of the seminal paper by Bardeen, Cooper, and Schrieffer (BCS) reporting their very successful theory of what are now called conventional low-temperature superconductors. The BCS paper provides a benchmark for what a successful theory should provide: an explanation of how superconductivity evolves out of the "normal" (i.e., nonsuperconducting) state that is found above $\mathrm{T}_{\mathrm{c}}$ and a model for the mechanism.

Comparison to the BCS theory highlights the major theoretical quandary of high- $T_{c}$ superconductivity. The normal state of the cuprates, unlike that of conventional materials, is itself a poorly understood "strange metal." In truly normal metals, like the copper used in house wiring, electrons show no significant correlation beyond what is imposed by their quantum (Fermi) statistics. Such metals are known as Fermi liquids after Enrico Fermi, who first identified how an assembly of a large number of independent electrons should behave. In contrast, the electrons in the strangemetal state are strongly correlated despite remaining liquid and repeatedly defy the expectations for a Fermi-liquid metal.

A theory of the strange metal is likely to be a key component in any successful theory of high-temperature superconductivity itself because it is out of the strange metal that the superconductivity arises. Since the identification of the strange metal in the cuprates, similar metallic "non-Fermi-liquid" behavior has been found in other electronic materials, lending further significance to the problem. While there are some exciting ideas and approaches to the problem-with exotic names like quantum criticality, marginal Fermiliquid theory, and dynamical mean-field theoryunderstanding the strange metal is a significant challenge to the physics and materials communities.

Most recent work on the cuprates centers on one part of the strange metal region of the phase diagram, known as the pseudo-gap regime. In this regime, materials are being pushed away from the optimal superconducting state toward the Mott insulator mentioned earlier- a fundamentally different state of matter that is insulating not because of band structure but because of interaction-induced electronic correlations. As described above, proximity to a Mott insulator seems to be a common source of complex behavior in quantum materials. Indeed, the full complexity of the pseudo-gap regime of the cuprates is only now beginning to be revealed. Early studies based on standard probes observed only that there seemed to be a gradual reduction in the number of low-energy excitations; the current name for the state is based on the similarity between these observations and the excitation gap of a superconductor.

However, in the early years of high-temperature-superconductivity studies, this region of the phase diagram could only be studied to a limited extent, owing to the lack of high-quality samples at low doping and of appropriate experimental probes. As a result of a Herculean effort by the community, this situation has dramatically changed, and both crystals of remarkable quality and a diverse range of high-resolution tools are now being employed to study the problem (see Sidebar "High-Temperature Superconductivity"). They have revealed an unanticipated diversity of phenomena.

Experiments on the pseudo-gap state have uncovered clear examples of coexisting electronic orders-usually referred to as "competing orders" in this community. Perhaps most dramatic are the scanning tunneling microscope images, which have shown intrinsically inhomogeneous energy spectra (Figure 4.4) and strong evidence of short-range electronic charge ordering. Such 


\section{HIGH-TEMPERATURE SUPERCONDUCTIVITY}

\section{Study of High-Temperature Superconductivity Has Revealed Exciting Nerw Physics and Stimulated Advances in Electron Spectroscopy}

High-temperature superconductivity in the cuprates rocked the scientific community upon its discovery in 1986 and has remained a major challenge to our understanding ever since. The occurrence of superconductivity-the flow of electric current without resistance-in such unconventional materials (ceramics instead of metals) at such high temperatures (the record at atmospheric pressure is $T_{c}=138 \mathrm{~K}$, as compared to typical values of $\mathrm{T}_{\mathrm{c}} \sim 10$ to $15 \mathrm{~K}$ in conventional materials) stimulated enormous scientific activity. As it became clear that a quick explanation would not be forthcoming, the importance of the problem led to steady development of difficult experimental techniques and improved materials. These advances facilitated scientific and technological progress much more broadly. Probably the techniques that have benefited most from two decades of high- $T_{c}$ research are spectroscopic probes of electrons: angle-resolved photoemission and scanning tunneling spectroscopy.

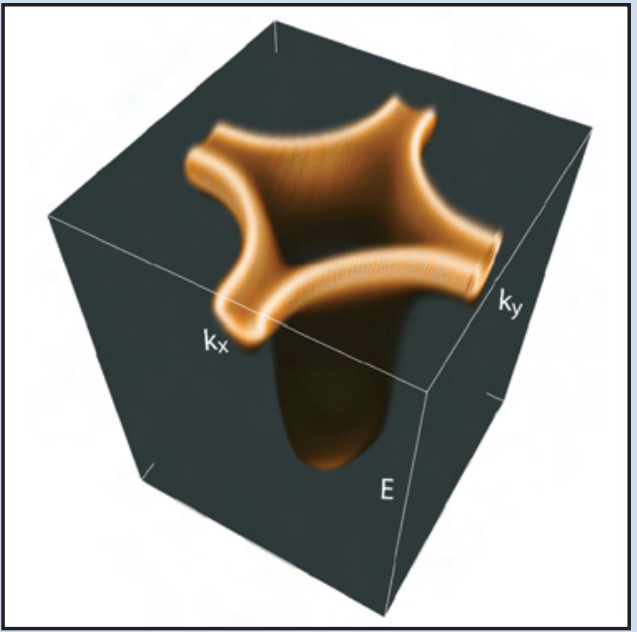

FIGURE 1. An image of the Fermi surface of the hightemperature superconductor $\mathrm{Bi}_{2} \mathrm{Sr}_{2} \mathrm{CaCu}_{2} \mathrm{O}_{8+\delta}$ reconstructed from ARPES data.

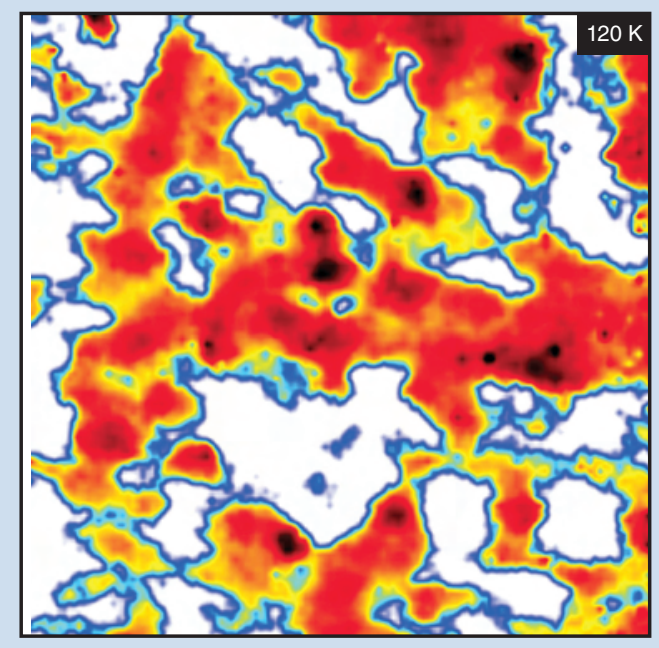

Angle-resolved photoemission spectroscopy (ARPES) is a technique to measure the distribution of energy and momentum of electrons in a material. High-intensity radiation (light) illuminates the sample. A photon absorbed by an electron gives the electron enough energy to escape from the crystal. By measuring the energy and momentum of the ejected electron and knowing these quantities for the incident photon, one can deduce the energy and momentum of the electron before it was scattered. Primarily because of the demands of the cuprates, the energy and angular (hence momentum) resolutions of ARPES have improved by roughly a factor of 100 and 20, respectively! In the cuprates, ARPES has allowed direct measurement of electronic scattering rates, Fermi surfaces, angular variation of the gap, and coupling of electrons to collective modes (see for example Figure 1). It is being applied much more broadly, to characterize novel materials such as $\mathrm{Sr}_{2} \mathrm{RuO}_{4}$, graphene, $\mathrm{MgB}_{2}$, heavy fermion and organic superconductors, and many others.

Development of several scanning probe techniques has been spurred by efforts to understand high-temperature superconductivity. These techniques enable an experimentalist to scan the surface of a sample and record many different properties, including local electronic structure, magnetic field, charge density, compressibility, and others. To make the dramatic observation shown in Figure 2 requires stabilizing the STM and sample at a much-elevated temperature compared to the $4.2-\mathrm{K}$ norm for these sorts of measurements,

FIGURE 2. Scanning tunneling microscope (STM) data on a hightemperature superconductor, demonstrating large local variations of the energy gap (energy required to add or remove an electron). The area shown is $30-n m$ square, and the color scale varies from a gap of zero (white) to approximately $100 \mathrm{meV}$ (black). Remarkably, this data was taken at $120 \mathrm{~K}$, well above the $93 \mathrm{~K}$ superconductingtransition temperature of this sample. Clearly some sort of gap persists into the normal state! 
a significant technical advance. STM measurements of high-temperature superconductors have separately identified and mapped the distributions of different types of impurities, dopants, and superconducting vortices; spectroscopically characterized the different electronic states; and revealed emergent spatial structures, such as checkerboard and stripe correlations. STMs and other scanning probes provide a powerful general tool to measure and manipulate a host of nanoscale structures and are used across an increasing number of scientific disciplines.

fluctuating charge order is present both below and above the superconducting critical temperature, $T_{c}$. Other experiments—nuclear magnetic resonance, muon spin resonance, and inelastic neutron scattering- have provided evidence for magnetic fluctuations and/or local magnetic ordering as well, even coexistence of antiferromagnetism and superconductivity.

Stimulated by experiments like these, both in the cuprates and in other quantum materials, theory seems to be converging on the notion that competing or coexisting orders are often the cause of emergent behaviors such as high-temperature superconductivity. This notion is also relevant to other superconductors, such as those based on the heavy-fermion compounds and organic materials. The key elements to understand are the nature of the competition and the relation between these orders, which would lead to a unifying description. This understanding would permit the community to model and calculate properties of materials in this regime. This challenge to theory remains open, and though there are ideas, they are controversial. Both new experiments and more theoretical work will be needed to elucidate the situation, followed by ideas for the creation of new, higher-performance materials.

\section{Correlated Quantum Liquids}

High-temperature superconductivity, with its non-Fermi-liquid normal state, focused attention on

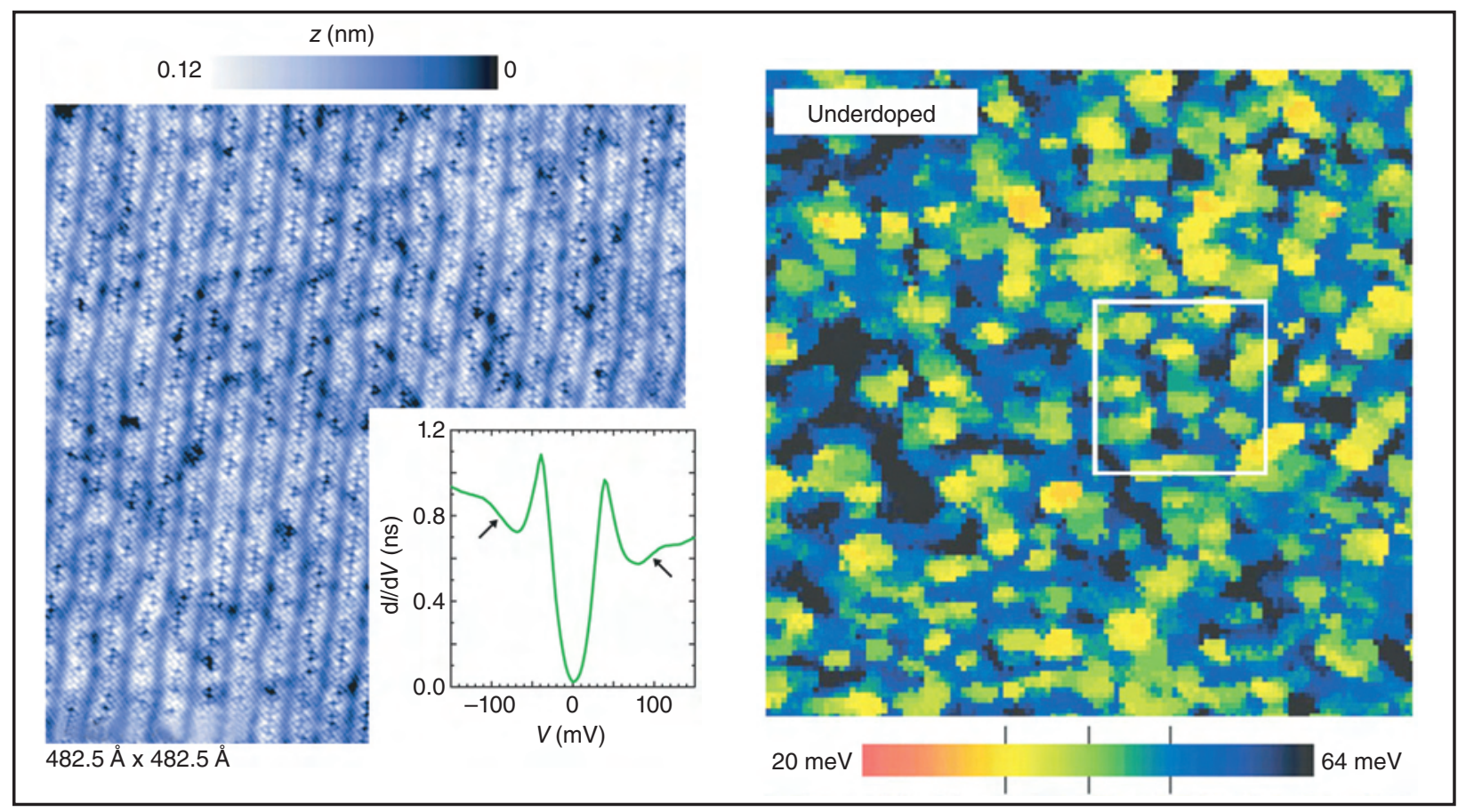

FIGURE 4.4. Spectroscopic imaging with atomic-scale spatial resolution is now possible. Left: Atomic-resolution scanning tunneling microscope image at $4.2 \mathrm{~K}$ of $\mathrm{BiSrCaCuO}$, a high-temperature superconductor (see Figure 1 in sidebar "High-Temperature Superconductivity). Right: A map of the superconducting gap. Since most chemical reactions start at surfaces, these images also hold the key to understanding the relationship between electronic and atomic structure in condensed matter. 
correlated quantum liquids more generally. It is now recognized theoretically and experimentally that unconventional quantum fluids arise in many guises. An interesting example is the thermoelectric (a material that generates an electrical voltage from an applied temperature gradient) $\mathrm{Na}_{\mathrm{x}} \mathrm{CoO}_{2}$, which has a very large thermopower for a sodium concentration $x>0.75$ and may be useful in low-temperature refrigeration applications (Figure 4.5). The high thermopower is directly related to strong correlations, which lead to a large spin entropy transported by the charge carriers. Effective room-temperature thermoelectrics have been made in superlattices combining two strongly correlated oxide materials, made using methods described later in this chapter.

Correlated liquids also occur in insulators, in which case the correlated particles are neutral, e.g., spins rather than charges. Examples of spin liquids are continually accumulating as more and more compounds with frustrated magnetism are being synthesized and studied (see Sidebar "Frustrated Magnetism"). A very exciting prospect, envisioned by Philip Anderson as early as 1974 , is the idea of a quantum spin

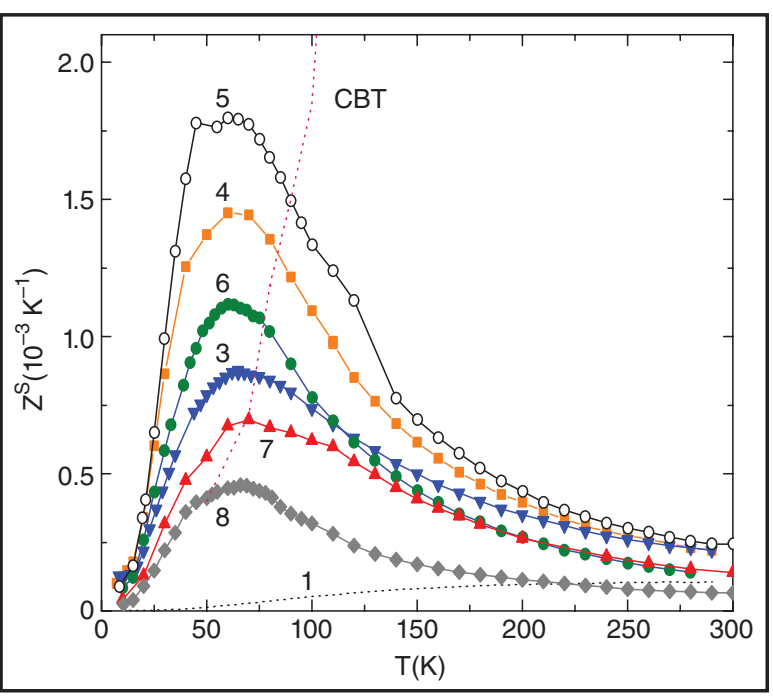

FIGURE 4.5. The thermoelectric figure of merit, $Z$, is shown versus temperature for $\mathrm{Na}_{\mathrm{x}} \mathrm{CoO}_{2}$, a strongly correlated material with hole-like conduction. The different curves result from tuning the sodium concentration, $x$, over a narrow range. This material shows one of the largest known $Z$ values for any hole-like material below $100 \mathrm{~K}$ (the best material CBT above $100 \mathrm{~K}$ is shown by the dashed curve). The large $\mathrm{Z}$ is a direct consequence of strong electron correlations. $\mathrm{Na}_{\mathrm{x}} \mathrm{CoO}_{2}$ is thus a promising candidate component for an electronic Peltiertype refrigerator for applications below $100 \mathrm{~K}$. liquid, in which the combination of frustration and quantum zero-point motion are sufficient to suppress magnetic order, even at zero temperature (absolute zero).

More than 30 years later, a number of very promising materials exhibiting such behavior have just recently begun to appear: $\mathrm{ZnCu}_{3}(\mathrm{OH})_{6} \mathrm{Cl}_{2}$, called herbertsmithite, a layered material whose spins become correlated below $300 \mathrm{~K}$, the so-called "Curie-Weiss temperature," but which does not order even at the lowest measured temperature of $50 \mathrm{mK} ; \mathrm{Na}_{4} \mathrm{Ir}_{3} \mathrm{O}_{8}$, where the Curie-Weiss temperature is greater than $600 \mathrm{~K}$ and there is no order above $2 \mathrm{~K}$; and several other organic and inorganic materials. Theory suggests that quantum spin liquids can exhibit quite remarkable properties, such as artificial photons-propagating excitations that behave just like light in a dielectric but are different from true electromagnetic waves—and fractional quasiparticles with exotic quantum statistics, which could in principle be an error-free foundation for quantum computing. Connecting the experimental examples to the theoretical proposals is a difficult challenge, but one with great intellectual and practical potential. In general, understanding quantum liquids is a key fundamental scientific issue in correlated materials.

\section{Coupling Spin and Charge}

Numerous new technologies could be enabled by materials in which charge-related properties-charge density, electric polarization, and currents-interact with spin-related (magnetic) ones-magnetization, spin currents, and spin waves. A simple example of an exciting device based on these phenomena is a giant magneto-resistance (GMR) spin valve, in which the electrical resistance is modulated by the orientation of a magnetic domain in a metallic material. Such GMR devices are already quite successful technologically for high-density information storage and represent a several-billion-dollar industry. Correlated electron materials offer many other means in which spin and charge can become coupled.

The colossal magneto-resistance (CMR) manganite materials display much larger resistance changes than GMR devices even within a single material. So far, their applications have been limited by materials properties and the fact that the CMR phenomena peaks 
below room temperature. However, the fundamental science of the manganites remains a fascinating subject with many open questions and presents a stimulus for new materials synthetic research. Many manganites display microscopic phase separation, but it is not known whether this is intrinsic or critical to the CMR. Some manganites are "half-metallic" ferromagnets, which means that only one spin polarization conducts, while the other is insulating. Since the current is then spin-polarized, this may be useful to inject currents of electron spin into other materials.

Other manganites behave as multiferroics at sufficiently low temperature. A multiferroic is a material that is simultaneously magnetic (sometimes exhibiting ferromagnetism and sometimes not) and ferroelectric. Similarly to a ferromagnet, which has a spontaneous magnetic dipole moment, a ferroelectric has a spontaneous electric dipole moment or electric polarization, usually as a result of a rearrangement of atomic ions in the crystal structure. Ferroelectrics are used in diverse applications from barbeque lighters to motion sensors and non-volatile memory for data storage. The combination of ferroelectricity and magnetism is, however, quite rare. When present, it can be an impressive example of correlation physics: magnetism arises from electron correlation, while ferroelectricity from correlation of charged ions, i.e., of the crystal lattice. Only recently have new classes of such multiferroics have been discovered, and an understanding of the interplay of these two types of orders has begun to be developed (see an example in the Sidebar "Frustrated Magnetism"). In some of these promising new materials, magnetism and ferroelectricity are strongly coupled. The prospect of multiferroic materials with strong magneto-electric coupling is also exciting from the point of view of applications, potentially allowing (see Figure 4.2) electrical control of magnetism, e.g., for information storage technology.

\section{Correlations in Heterostructures}

While we have emphasized that correlations in conventional semiconductors are ordinarily weak, much stronger correlations appear in semiconductor heterostructures (artificial structures of two or more materials). As a result of their many applications, semiconductors have been the subject of vast R\&D investments aimed at developing materials of unprecedented purity and structural quality, as well as methods to process them with exquisite control, in order to optimize device performance and production yields. These investments have also paid off for fundamental science in a number of ways and will continue to do so. More recently, major progress has been made, largely outside the United States, in fabrication of high-quality heterostructures made from strongly correlated materials. These strongly correlated heterostructures (see Sidebar "Correlated Interfaces") can be used to create devices that surpass some limitations of current semiconductor devices.

Several remarkable examples of electronic correlations were discovered via the creation of nearly ideal twodimensional electron gases (2DEGs) at the interfaces between $\mathrm{GaAs}$ and $\mathrm{AlGaAs}$. In low-temperature experiments, such 2DEGs have provided the setting for the observation of the integral and fractional quantum Hall effects (which reflect complex correlated states that appear in high magnetic fields) and for the observation of quantum-interference effects such as weak localization. When further confining potentials are applied within the plane of such a 2DEG, lower-dimensionality structures are created with high reproducibility. This has given rise to the observation of conductance quantization in narrow constrictions (quantum point contacts), and Luttinger-liquid behavior (a kind of one-dimensional strange metal) in quantum wires, and a great variety of effects. In quantum wires and quantum dots-fully confined structures in which the number of electrons can be precisely controlled-effects such as the Coulomb blockade and the Kondo effect (both discussed in Chapter 6) and the quantum manifestations of classical chaos have been observed.

The GaAs/AlGaAs 2DEG will remain a key platform for fundamental physics research in the foreseeable future, because of its availability, flexibility, and quality. For these reasons, it is one of the best systems to explore controllability of electronic quantum correlations, one possible application of which would be quantum information processing. Some of the most challenging problems in this direction are:

- Control and manipulation of individual electron spins. In quantum-dot structures, it has been shown possible to manipulate and measure the 


\section{CORRELATED INTERFACES}

\section{Interfaces between Correlated-Electron Materials - the First Step toward Semiconductor-Type Heterostructures-Have Been Made and Show New Behavior Not Found in their Semiconductor Counterparts.}

Experimenters in recent years have been taking the first steps toward semiconductor-style heterostructures (devices made by using precisely spatially modulated chemistry) grown from correlated electronic materials, involving $\mathrm{d}$ or f electrons. Ultimately, such devices might allow one to combine the flexibility of semiconductor processing with the added capabilities of these materials due to collective phenomena like magnetism or superconductivity. In fact, the latest experiments show that these devices can show even more functionality that does not show up in bulk ma-

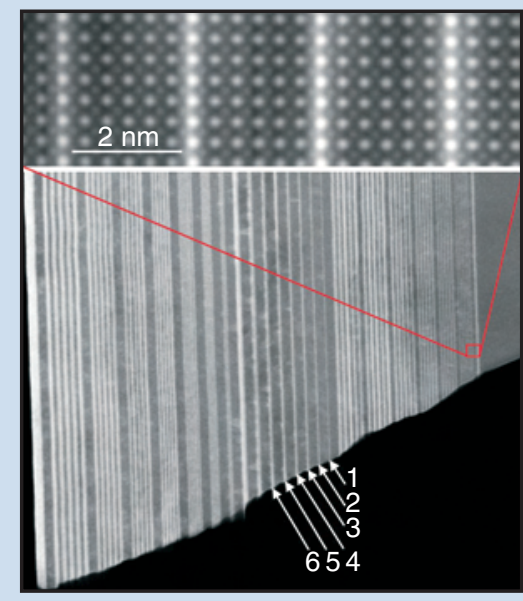

FIGURE 1. Image of a structure consisting of $\mathrm{SrTiO}_{3}$ (dark layers) and $\mathrm{LaTiO}_{3}$ (bright layers) taken by scanning transmission electron microscopy.

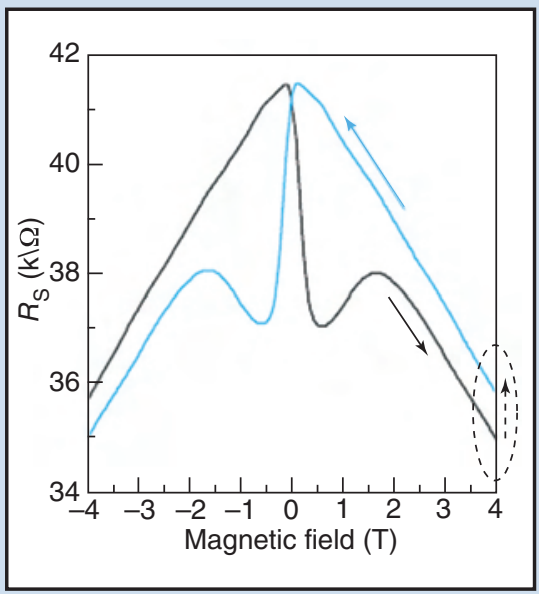

FIGURE 2. Graph showing hysteresis in the sheet resistance of the two-dimensional electron gas at the interface between $\mathrm{SrTiO}_{3}$ and $\mathrm{LaAlO}_{3}$, strikingly similar to that seen for ferromagnetic metals. terials.

Consider a structure consisting of $\mathrm{SrTiO}_{3}$ (dark layers) and $\mathrm{LaTiO}_{3}$ (bright layers) taken by scanning transmission electron microscopy (Figure 1). In bulk crystalline form, $\mathrm{SrTiO}_{3}$ is a "band insulator" with unusual dielectric properties, while $\mathrm{LaTiO}_{3}$ is an antiferromagnetic strongly correlated Mott insulator. Remarkably, charge transfer between the two materials results in a conducting two-dimensional electron gas at the interface, despite the fact that both materials are insulators.

Other interfaces of this type are under increasing investigation. The interface of $\mathrm{SrTiO}_{3}$ and $\mathrm{LaAlO}_{3}$, two correlated band insulators, also gives rise to a conducting interfacial electron gas. In this case the measured conductivity indicates a very high quality. The carrier mobility, the the velocity induced per unit applied electric field, was higher than $10,000 \mathrm{~cm}^{2} / \mathrm{Vs}$, comparable to good quality electron gases in GaAs.

Very recent experiments suggest that the interface between $\mathrm{SrTiO}_{3}$ and $\mathrm{LaAlO}_{3}$ is not only conducting, it is also magnetic. Even more so than the conductivity, this is surprising since neither material is magnetic. Figure 2 shows hysteresis in the sheet resistance of the interfacial electron gas, strikingly similar to that seen for ferromagnetic metals. It is likely that, as proposed by the experimenters and supported by theoretical calculations, electrons are transferred at the interface into the $\mathrm{Ti} d$ orbitals. Then, because of the strong Coulomb repulsion in these orbitals, they become correlated and magnetic. Other signs of classic correlation phenomena like the Kondo effect were also observed in the same study. It is intriguing to consider the possibilities of combining ferromagnetism and two-dimensional electron transport in this structure, which is completely different from the diluted magnetic semiconductors that have been intensively studied in the last decade. 
Another very different effect is observed in a related but distinct structure: an electron gas localized about a single unit-cell-thick layer of $\mathrm{SrTi}_{0.8} \mathrm{Nb}_{0.2} \mathrm{O}_{3}$ embedded inside bulk $\mathrm{SrTiO}_{3}$. This electron gas was found to show an enhanced thermoelectric effect, i.e., generation of a voltage by a temperature gradient. Its Seebeck coefficient-the ratio of the induced voltage to the temperature gradient-was observed to be approximately five times larger than in bulk $\mathrm{SrTiO}_{3}$ (see Figure 3). Such large values could make these kinds of structures useful in diverse thermoelectric applications.

Activity on correlated electron interfaces in on the increase, though almost all fabrication of these structures is taking place outside the United States. Given the richness of collective phenomena in correlated materials, the spectrum of science to be explored in such layered structures is almost limitless.

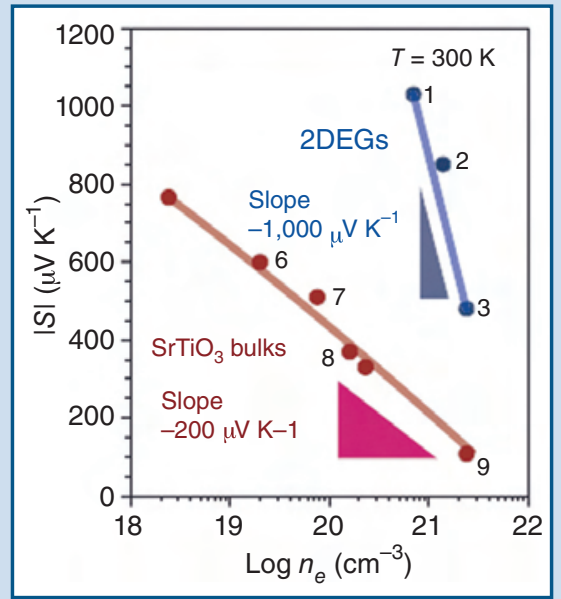

FIGURE 3. Plot of the absolute value of the Seebeck coefficient shows that the two-dimensional electron gases at the interface (2DEGs) have an enhanced thermoelectric effect relative to bulk $\mathrm{SrTiO}_{3}$. real-time dynamics of one or two confined electron and many nuclear spins by electrical (see Figure 4.6) and/or optical (Chapter 2) means. Can this be extended to create correlated "entangled" quantum states (see Sidebar "Entanglement" in Chapter 2) of several electronic and/or nuclear spins in a controlled way?

- Detection of "non-Abelian" fractional quantum Hall states. These are a more exotic version of the established fractional quantum Hall states, and are predicted by theory but only hinted at by current experiments. If these could be observed and controlled at the level of manipulation of individual particle-like excitations, the unique properties of these excitations are believed to offer a faulttolerant method of quantum computing.

- Spintronics of mobile carriers. Most electronic devices operate by manipulating local charge densities and electrical currents. However, electrons also carry spin, and the experimental means to manipulate spin densities and spin currents in semiconductors has been developing extremely rapidly in the past decade. The complexity of spin dynamics and transport is, however, vastly higher than for conventional charge transport, particularly when large-spin ions such as manganese are added to the heterostructure.
Along with continued exploration of semiconductor heterostructures, heterostructures fabricated from two or more strongly correlated materials provide a very powerful path to devices in which spin, charge, and atomic structure are intricately interwoven and can be controlled independently. These are already leading to improvements of materials such as thermoelectrics, in which optimization requires independent control of thermal and electrical transport properties.

\section{CORRELATION PHENOMENA BEYOND ELECTRONS}

\section{Ultra-Cold Atomic Gases}

The last decade has witnessed several remarkable experimental advances in trapping and cooling ultracold atomic gases to extremely low temperatures (microkelvin or even nanokelvin). The interest in doing research on ultra-cold atoms has skyrocketed, and the manipulations of the trapped atoms are increasingly sophisticated. So far, the majority of experiments in these systems has focused on condensates, a quantum phenomena in which, as in a superconductor, a macroscopic number of particles occupy a single quantum state extending over the entire atomic cloud (see the discussion of coherence in Chapter 2). 


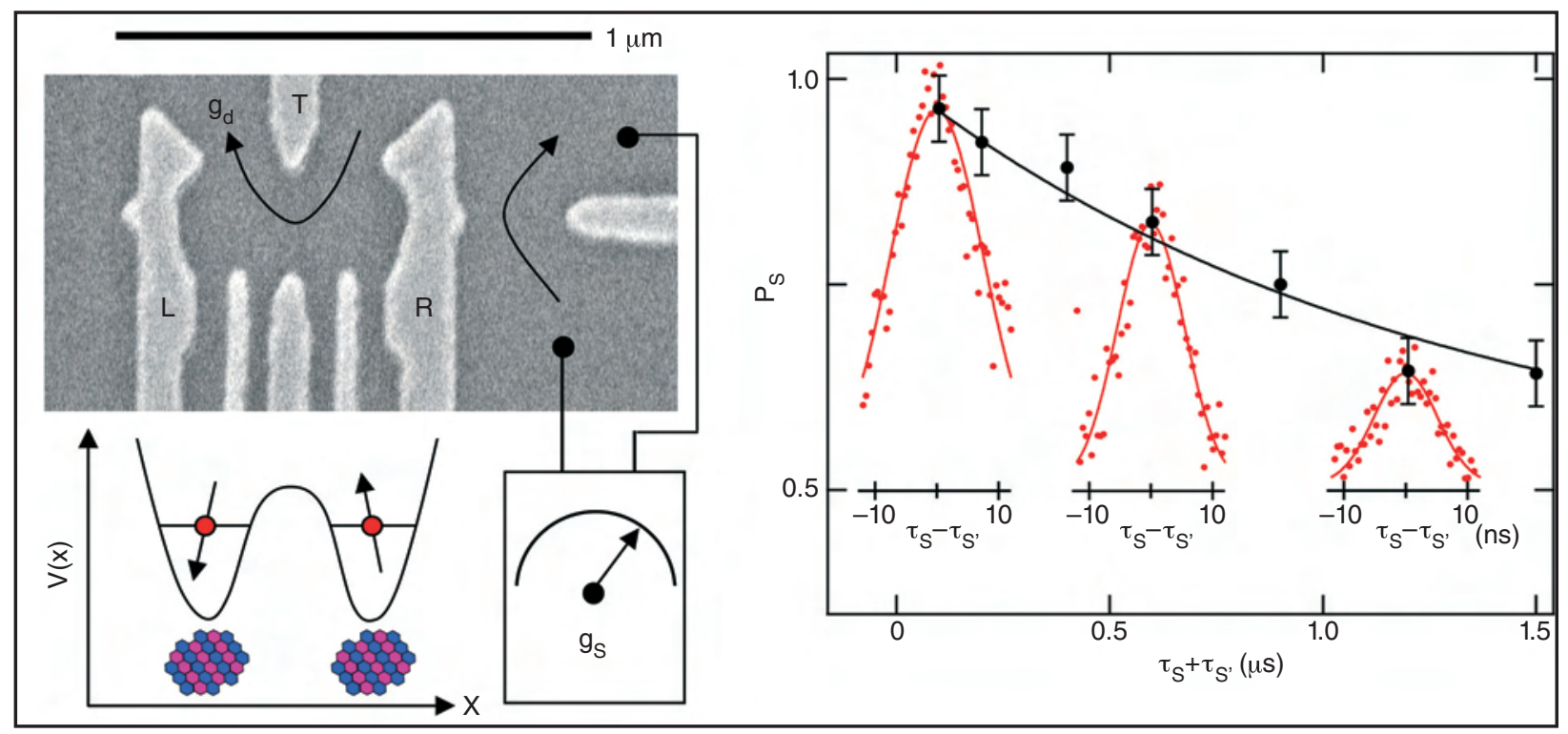

FIGURE 4.6. Left: Scanning electron microscope image and corresponding schematic of a double-quantum-dot device in a GaAs/AlGaAs heterostructure that can be tuned to contain one electron in each dot (potential well in the schematic). The channel on the right (labeled $g_{s}$ in the schematic) is a "quantum-point-contact" charge detector used for the measurement scheme. The spins of the electrons precess, owing to interaction with the many nuclear magnetic moments in the material. Right: By utilizing fast voltage pulse sequences, it is possible to remove the main influence of this precession, extending the "lifetime" of a specific (singlet) spin state a thousand-fold from a few nanoseconds to approximately a microsecond. Plotted is the probability of remaining in this state as a function of time for different pulse sequences.

For bosonic atoms, quantum degeneracy leads to Bose-Einstein condensation, similar in general terms to the superfluid transition in ${ }^{4} \mathrm{He}$. For fermionic atoms, quantum degeneracy and atomic interactions lead to a condensate of Cooper pairs of atoms, in the same way as electrons form Cooper pairs in a conventional superconductor. The tools of atomic physics discussed in Chapter 2 can be used to manipulate these states in ways that are impossible with either liquid helium or superconducting solids. One example is that the superfluid state made from fermionic alkali atoms can be tuned continuously between a Bose condensate of diatomic molecules and a Cooper-paired state analogous to superconductivity. Another is that superfluids of bosonic atoms with spin ("spinor Bose condensates") can be tuned between states that combine magnetic and superfluid order in different ways.

A trend for the future in this field is a move from "simple" condensate formation toward situations in which the atoms are strongly correlated. This can be accomplished by suppressing kinetic energy with an optical potential; it can also be done by strengthening inter-atomic interactions by tuning to an atomic "Feshbach resonance" or using dipolar atoms with long-range interactions. A significant community is developing at the interface of condensed-matter and atomic physics, combining the theoretical expertise in correlation physics from the former with the experimental methods and quantum information perspective of the latter. The result is an ever-increasing ability to control quantum correlations in these systems. This is possible because the atoms in these systems are so well separated that their internal quantum states remain largely unmodified by their environment and thus can be well understood and precisely controlled through laser excitation. Another significant advantage is that it is frequently easier to observe the real-time quantum dynamics in these systems than in solid-state materials because they are so much slower.

A major effort for the future is the creation of optical lattice emulators, in which challenging problems in correlated electron physics are attacked by building atomic analogues. An optical lattice created by interference of laser beams is used to replicate the lattice of a 
solid by trapping atoms preferentially at the lattice points. A precisely engineered atomic system could, for instance, answer the still controversial question of whether the Hubbard model (a popular starting point for theory of high-temperature superconductivity) contains the right physics to describe the cuprate materials. Ultimately, such quantum emulators could address a multitude of problems in which ordinary computational studies are prohibitively costly. By engineering systems with interactions different from any crystalline material, it may also be possible to produce novel quantum states with properties not found in solids but desirable from a fundamental scientific or a technical point of view. For example, one might be able to produce conjectured "topological" states of matter whose exotic excitations encode information in a highly non-local way useful for encryption or computation.

The key challenge in progressing toward the above goals is developing new probes and trapping methods for ultra-cold atoms. Currently most of the probes used to determine the state of an ultra-cold atomic gas are not directly comparable to the standard probes used to determine correlated structure in solids. For example, rather than directly measuring momentum-space properties as in neutron or photon scattering in solids, atomic physicists measure the momentum distribution destructively by releasing the condensate, allowing the atoms to strike a distant screen, and interpreting the real-space distribution on the screen to provide information about the original momentum-space distribution. This method is sufficient to determine basic properties such as superfluidity; however, advances in measurement would enable many more quantitative comparisons between electronic and atomic systems.

\section{Soft Matter}

The ubiquity of strong correlations is not limited to quantum phenomena in hard inorganic and organic crystalline solids. Indeed, complex structures and behaviors appear with great regularity in soft materials, which we take to mean materials built from particles larger than atoms or small molecules, e.g., organic constituents, polymers/macromolecules, or even micron-sized particles or grains (see Chapter 3). Strong correlations can be induced by the geometric constraints from the connectivity of the large particles involved, by strong electrostatic interactions of ionized constituents, or by hydrodynamic effects of the fluids involved. Because the characteristic interaction energies are also significantly lower and often comparable to the available thermal energy at room temperature, thermal fluctuations are more significant, and quantum effects are usually negligible.

Equilibrium correlations in soft matter. Problems in soft matter are very diverse, reflecting the strong interdisciplinary nature of the field and interactions between the scientific and engineering communities in these areas. An interesting and important example with broad relevance is the physics of screening in ionic fluids. Many important structures in biological systems are significantly charged in the cellular fluid. For example, DNA carries a (negative) charge of approximately 1 electron per 3 Ångstroms of its length. However, the DNA must often condense into a very compact form, which requires screening of this charge. Remarkably, under many circumstances, over-screening can occur, resulting in an effective net attraction between likecharged objects (see Figure 4.7) such as DNA or actin filaments! It can be shown that if there are no correlations between the ionic screening charges, such attraction is impossible, and generally it is believed that such attractive forces are the result of highly correlated screening layers very close to the structures being attracted. An understanding of such correlated screening

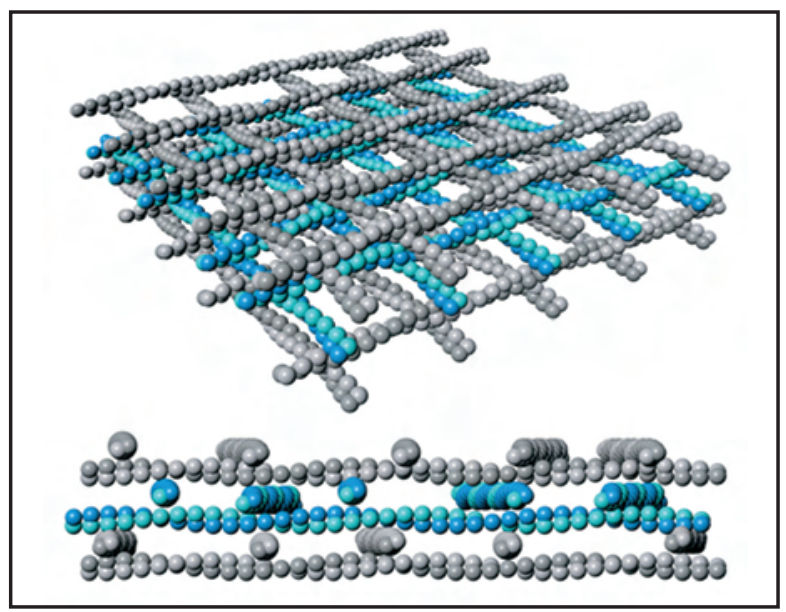

FIGURE 4.7. Schematic illustration of "raft" of actin filaments that forms as the result of a short-range attraction, despite the fact that all actin filaments have the same (negative) charge and would naively be expected to repel one another. The attraction is mediated by counter ions in the solution. 
in a sufficiently general context to be useful to biologically relevant modeling or design of drug delivery systems is a great challenge to theory and experiment.

Non-equilibrium soft matter. Because the molecular building blocks of soft matter are much larger than atoms, generally the microscopic "rearrangement time" is much longer than in hard materials. Moreover, all motions are essentially classical. Without quantum tunneling, the molecules cannot pass through energy barriers in their motion, but must pass over them by thermal activation, which is often a slower process than in hard materials because of the longer rearrangement time. When strong geometrical constraints are present, this longer rearrangement time often leads to the material falling out of equilibrium. In the extreme case, when the particles involved are large compared to atomic or molecular scales, for instance grains in a sand pile, the forces between them can be so strong that thermal agitation is completely negligible, and the system is never even close to equilibrium (see the discussion of "jamming" in Chapter 6).

This general class of phenomena is of great fundamental and practical importance. Structural glasses, materials that exhibit a shear modulus like a solid but are amorphous rather than crystalline, are extremely common over a wide array of chemical constituents, ranging over silica (window) glasses, metallic glasses, polymer glasses, and a variety of soft materials made from larger constituents like polystyrene spheres (see Chapter 3). They exhibit behavior intermediate between that of solids and liquids, and respond to stresses non-linearly and over a tremendous range of time scales. For example, for some materials super-cooling through the glass transition yields a striking range of viscosities that vary a billion-fold over a narrow range of temperatures. The nature of this growth remains a challenge to understand. Failure modes-fracture, crack propagation, and plastic deformation - in amorphous and composite solids are critical to their performance, and their strength can often exceed that of similar crystalline solids. Understanding in this area is vital to improving the performance characteristics of the composite materials now used, for example, in the aerospace industry. Even empirical models for such behavior are not well developed. Advances in experimental probes, such as imaging of individual particle motions in these materials, when coupled with improvements in theoretical models, promise major progress in these important challenges over the upcoming decades. While there are many further examples of equal significance, we defer their discussion to Chapter 6, which focuses entirely on non-equilibrium phenomena.

\section{Collective Phenomena in Biology}

Many of the most challenging problems at the frontier of modern biology require understanding how complex collective phenomena emerge from a large number of relatively simple constituents. Certainly biological organisms, to function, involve correlated interactions of an enormous number of constituents. One feature distinguishing biological systems from the materials discussed earlier in this chapter is their complexity. For example, the number of distinct types of proteins in a cell typically is much larger than the number of distinct types of atoms in a non-biological solid. In addition, while correlated materials may involve several distinct length or energy scales, in biology this is carried to an extreme (see Sidebar "Correlated Biological Materials”). Finally, biological systems are always far from equilibrium (though in some limited situations some biological subsystems may be in local quasi-equilibrium). Thus, it is clear that understanding practically anything about functional mechanisms in a cell or in a higher-level structure from the microscopic degrees of freedom is a very challenging correlation problem! Until recently, this could be viewed largely as an academic observation. Now, with the dramatic advances in understanding the details of molecular biochemistry and bioinformatics, there may be sufficient microscopic knowledge that the problem of understanding the origin of collective phenomena in biological systems can be profitably studied.

At present, such a "mechanical" understanding of collective biological properties and processes is extremely limited. One area where significant progress has been made recently is the biophysics and biochemistry of single molecules. Tools such as "optical 


\section{CORRELATED BIOLOGICAL MATERIALS}

Like many engineered systems, a signature of biological systems is the precise, repeatable placement of its constituents in complex working machines. Unlike engineered objects such as cars, however, biological systems selforganize, develop, and age following rules encoded by the physics and chemistry initiated by the complement of biomolecules, many already precisely arranged, in the zygote. While the basic physics that drives these processes is largely the familiar macroscopic and mesoscopic kinetics and thermodynamics that we are comfortable thinking about, the complexity of the interactions, their nonlinearities, and their inhomogeneities prevent us from understanding how the cooperation of the innumerable chemical processes in a single cell is elaborated into an adult animal.

There are processes, however, that have begun to be uncovered that translate Ångstrom-scale correlations in structure and dynamics to the macroscopic function of entire organs. One clear example is the quasi-periodic beating of the human heart (Figure 1). The heart itself is, of course, a central organ in the life of many creatures. Its structure and placement in the body of the animal is critical to its proper function, and even small defects can lead to nonoptimal functioning of the organism and early death. The heart beats in a nearly periodic rhythm. However, far from being a simple oscillator, these rhythms can have a complex temporal and spatial structure. Different amounts of temporal and spatial correlation in the heart rate are indicative of different heart abnormalities and disease states.

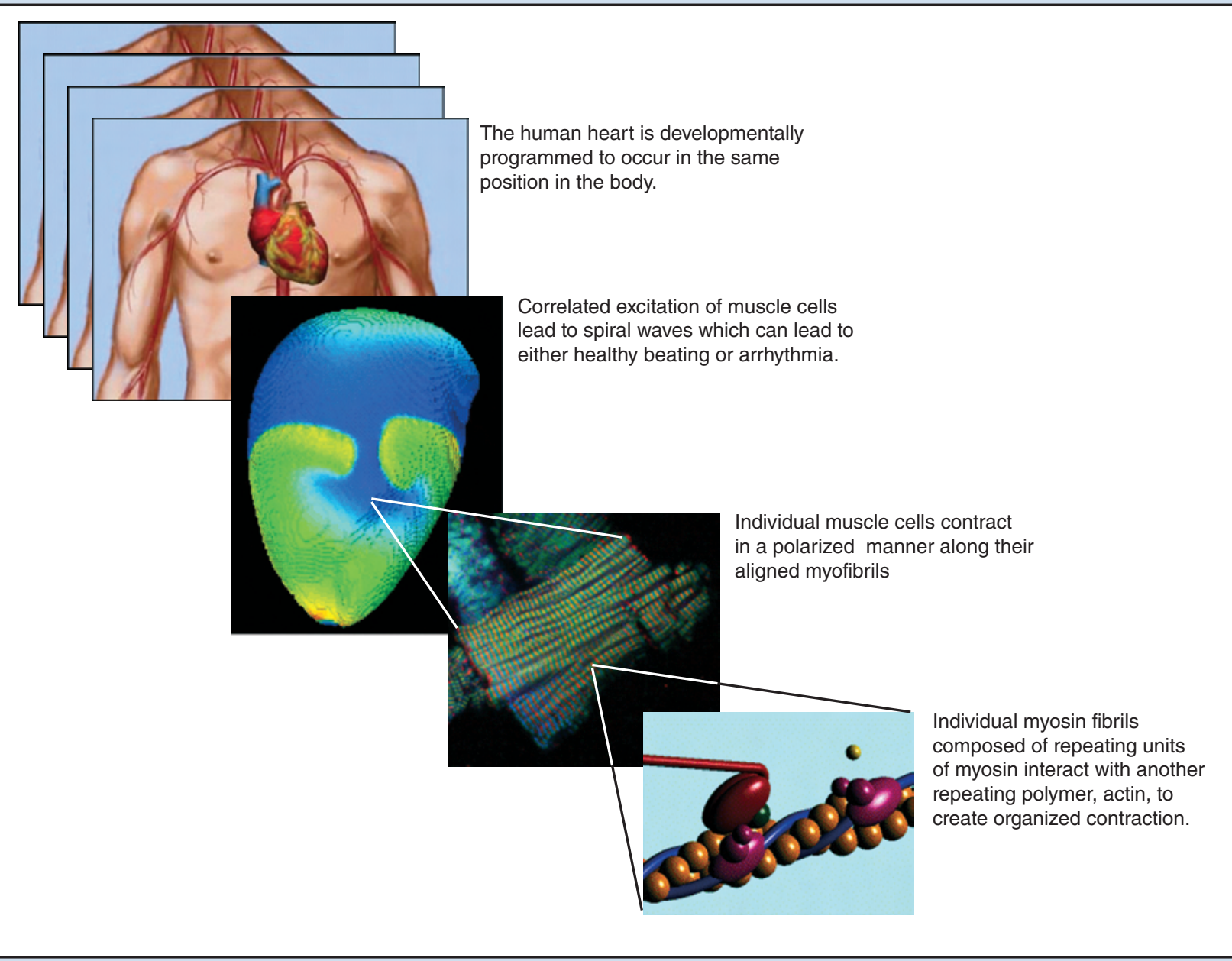

FIGURE 1. Correlations propagating upwards to successively larger length scales and longer time scales result in a beating heart. 
Electrical activity of the pace-maker cells of the sino-atrial (SA) node trigger action potentials that propagate along the atria toward the atrio-ventricular node. When the potentials reach the node, it triggers Purkinje fibers to deliver stimuli in a precise spatially and temporally controlled manner to cause a concerted contraction of the heart muscle. The contraction forces blood along the right paths through the internal chambers of the heart and into the vascular system. The electric wave is sensed by individual cardiomyocytes. When stimulated, each individual cell can contract, and it is the correlated contraction of cells in time that make a good heart beat.

The cell contraction itself is carried out by the motion of organized proteins, actin and myosin, that are organized into complex network structures and long, aligned fibrils. Myosin motors move the actin filaments relative to each other in an energy-dependent manner, resulting in a precise ratcheting motion. Recent studies have done much to demonstrate how Brownian dynamics and the correlated structure of the protein filaments collude to create efficient directed movement. When these Ångstrom-level correlated movements occurring within microseconds propagate to higher length and time scales, the result is strong contraction ( milliseconds) of single cells $(\sim 0.1 \mathrm{~mm})$ and their coordination across macroscopic tissue to create a healthy beating ( seconds) heart ( centimeters) that is precisely positioned and developed in a healthy animal.

The fundamental challenges still remain the ability to measure these effects dynamically in situ at the angstrom scale and to develop the means to rigorously incorporate nanoscale dynamics into micro- and macro-scale models of biological function. Further, it is rapidly becoming possible to genetically manipulate biological systems in very sophisticated ways. However, our ability to develop ab initio, biomolecular systems with the same scale and efficiency as biomolecular motors and with the ability to perform such complex actions as those of cells and animals is lacking, owing to a poor theoretical basis for dealing with highly structured, inhomogeneous materials such as these.

tweezers" are used to stretch single RNA molecules and understand their mechanical properties, which are related to their sequences. Measuring the work done in stretching the molecule rapidly, starting from an ensemble of initial conditions, enables an experimental test of the non-equilibrium work relations and remains an active topic of research in statistical physics (see the Sidebar "Fluctuation Theorems" in Chapter 6). Electrostatic phenomena have been studied (as described above in the soft-matter section) and may be related to the assembly of DNA and histones into chromosomes. The ratcheting action of muscle fibers is another active topic, in which the function of molecular motors called myosins are being studied. These are closely related to basic questions about nanoscale mechanics and statistical physics. Physicists and chemists are attempting to construct inorganic molecular motors that are as small, efficient, and robust as biological examples, and to quantify how close biological nanomotors are to fundamental limits implied by quantum mechanics and thermodynamics. Many other examples of correlations in biological sciences can be found elsewhere in this document, especially in Chapters 5 and 6, but the quantitative understanding of collective phenomena in biology is very much in its infancy.

\section{WHAT ARE THE NEEDS?}

To address the grand challenge of correlation phenomena, a concerted effort is required in three broad areas: materials synthesis, experimental measurement and control, and theoretical analysis. We will discuss each in turn below, with some specific examples. In all of these directions, it is important to recognize that truly fundamental challenges require non-traditional approaches that often develop gradually only by longterm research over an extended period. For example, as described earlier in this chapter, high-temperature superconductivity has been a very productive research area, but progress has occurred over a 20 -year period through the hard work of steadily improving experi- 
mental tools, materials, and theoretical techniques. While each step in this process has yielded its own dividends, it is the long view held by many key researchers that has enabled this field to progress. Progress in such frontier areas also requires collaborative, cross-disciplinary research modes.

Materials synthesis is a key enabler for advances in correlation science. The example of the physics of twodimensional electron gases at semiconductor interfaces has already been highlighted. The pervasiveness of these materials in science and technology is a consequence of a long-term, largely industrial, effort to grow them with ultra-high purity and in diverse geometries, and this has paid enormous scientific dividends. To successfully study and take advantage of strong correlations requires a similar ability to "design" and grow the appropriate samples (with consistently high quality and often in single crystalline form) for a much broader range of materials. For instance, there are many types of probes whose full informative potential can only be realized on large, single-crystal samples. Notable examples are neutron and angle-resolved electron photoemission spectroscopy. Other phenomena only appear when the defects of the material are reduced below some critical concentration.

This type of materials development cannot be done "on the side." Rather, it requires a continuity of effort and expertise over many years to improve quality and overcome hurdles involved in the highly non-equilibrium processes needed to grow most interesting solids. As discussed in Chapter 3, non-traditional approaches to materials growth, including biologically inspired selfassembly schemes may ultimately lead to a step change in this area and should be explored in parallel with traditional materials-science methods. A discussion of how to address the need for improvements in crystal and heterostructure growth is given in Chapter 7 .

An equally important requirement for unlocking the puzzles of emergent phenomena is the creation of new tools for measuring correlations in the laboratory. While some correlations, such as the alignment of spins in a ferromagnet, are easily identified by such macroscopic signatures as a spontaneous magnetic moment, others like the antiferromagnetic order discussed earlier in this chapter are more subtle and require specific tools to uncover them. Continued development of new and improved experimental probes for complex magnetism, including scanning probes and $\mathrm{x}$-ray nanoprobes, remains critical to observing new types of correlations.

Some directions for such development are clear. Spectroscopy and scattering using photons (x-rays), neutrons, and electrons should be pushed to ever higher resolution in momentum, energy, and space for imaging. As discussed earlier, electron photoemission spectroscopy has advanced dramatically in resolution in the last two decades to the point where it can be used to directly measure the Fermi surface of low-energy electron excitations in a variety of materials. This can be directly compared with theoretical electronic structure predictions. Further enhancement of resolution will enable a fuller characterization of the photoelectron wave function and scattering rates. Scanning microscopies such as scanning tunneling microscopy (STM), atomic force microscopy (AFM), and others have enjoyed tremendous development in recent years. These now provide a probe of local electronic and atomic structures at surfaces of crystals, molecules, and other structures. Continued development of scanning probes such as chemically sensitive AFM and spinpolarized STM will allow even more detailed correlations to be measured with atomic precision. Another major direction is the development of optical methods to study real-time dynamics of fast quantum processes in molecules and solids (Chapter 2).

Improved theoretical methodology is a necessary partner to the above materials and experimental efforts. The ultimate goal of theory is to provide an accurate, predictive guide to the properties of materials and structures, both to enable rational design of new synthesis efforts and to explain fundamental mechanisms in natural systems. In strongly correlated situations, the full process of deducing macroscopic behavior from known microscopic laws-e.g., determining the magneto-electric response of a complex crystalline solid from the basic equations of quantum mechanics-is an extremely difficult problem.

In the realm of solid-state physics, the most common of such ab initio ("from first principles") methods is density functional theory in the local 
density approximation (LDA). LDA and its many descendants are designed essentially to capture the electronic structure of energy bands and basic structural parameters. In weakly correlated solids, they are quite successful. While this is an impressive achievement, it is clear that LDA-derived methods have inherent difficulties in capturing strong electron correlations. Recent attempts to combine LDA with dynamical mean-field theory (DMFT) partially address this problem. Even with such advanced corrections, LDA-derived methods are best suited for situations in which there is really a single characteristic length or energy scale; that is, in which a relatively small volume of the solid suffices to approximately reproduce the properties of the whole. However, among correlation problems of greatest interest, this condition is often not the case, and there are coupled, physically distinct processes occurring on a broad range of energy and length scales.

For example, in most magnetic materials, the exchange interactions which couple localized electron spins (typically, 1-500 K) are orders of magnitude smaller than the widths of the energy bands closest to the Fermi energy (usually of order 12,000 K = $1 \mathrm{eV}$ ), which represent the relevant (kinetic) energies of the conduction electrons. Frustration, described earlier, can suppress ordering to even lower temperature. Frequently, the competition between different interactions in correlated electronic materials leads to ordering at temperatures much below electronic energy scales. Competing and coexisting orders as observed experimentally indicate that even physics on tens of nanometer scales can be quite distinct from that within a few crystalline unit cells in materials such as the high- $T_{c}$ cuprates or the manganites. In semiconductors at millikelvin temperatures, new collective phenomena occur because of energy differences thousands of times smaller than the typical kinetic or potential energy of electrons. In all these cases, the more subtle nature of the low-energy physics requires the development of distinct and novel theoretical approaches of a more phenomenological nature. Such phenomenological studies are also important in providing unifying conceptual frameworks for new types of emergent behavior that cannot be obtained from ab initio methods and occur on scales that vary from the quantum world on up to macroscopic biological systems.

If one moves outside the realm of electronic materials, the examples of "simple" systems with only one characteristic energy or length scale are even scarcer (practically non-existent). Failure and plasticity in solids involve atomic-scale rearrangements, motion of large defects such as dislocations, grain boundaries, or cracks, and long-wavelength elastic waves. In biology, even a simple organism makes use of a vast hierarchy of interacting constituents from the DNA and proteins up to organs and tissues, and there is feedback from the largest scales (the organisms) to the DNA through evolution. Thus, in most correlation phenomena, theory must deal with the existence of important processes over a broad range of length and energy scales. It is not practical to expect a single theoretical or computational framework to derive all this behavior from first principles. Instead, it is critical for theory to embrace modeling on multiple scales, combining different tools to connect the distinct scales.

In electronic materials, this means combining results from LDA-type methods with phenomenological "effective-Hamiltonian" modeling to understand low-energy behavior. Even nominally simple effective models like the Hubbard model popular in studies of the high-temperature superconductors contain several distinct energy and length scales. Thus, even at this already reduced level of complexity, such problems may require a combination of approaches for their understanding. Recent developments of powerful "cluster" methods seem likely to describe much of the short-distance, high-energy physics. How this is connected to the ultimate low-energy behavior (e.g., transport properties, magnetism, and superconductivity) remains a largely open problem. It is important that these largely numerical methods-which, although considerably improved in recent years, remain inherently rather local - be carried out in concert with low-energy approaches that can properly describe global behavior.

Conversely, in most correlated electronic materials, the microscopic spin and orbital level structure is not 
obvious, and the interactions between them are even less so. Despite the deep puzzles therein, the hightemperature cuprates are a model of simplicity when compared with most correlated electron materials.

Thus, phenomenological approaches need input from first-principles or quantum-chemical methods to find an appropriate starting point. Much more interaction and collaboration between the ab initio and strongly correlated electron communities is needed to face the major challenges in these materials.

\section{CONCLUSIONS}

In this chapter, we have reviewed the Grand Challenge of understanding and controlling strong correlations and collective behavior in the basic energy sciences. We have seen that correlation phenomena are integral to fundamental scientific issues throughout this area and that their consequences can be highly enabling for improved functionality of materials and other structures. Advances in materials synthesis, experimental tools, and theoretical analysis are required if we are to take on this Challenge with a reasonable probability of success. 


\section{REALIZING THE DREAM OF NANOSCIENCE: ENERGY AND INFORMATION ON THE NANOSCALE}

The Grand Challenge for Nanoscience is to master energy and information on the nanoscale to create new technologies with capabilities rivaling those of living systems.

We find ourselves at the convergence of two nanoscience revolutions. Design and fabrication of devices on the nanometer scale, often based on solidstate electronic materials, is becoming possible. This is probably the first thing that comes to mind when one thinks of nanoscience. However, as we delve ever deeper into the world of biology, we find that biology is also based on a vast array of nanoscale mechanisms. Living systems are built of the same fundamental elements of matter and follow the same laws of physics as all inorganic and human engineered entities. Biological energy and chemical transduction, communication, adaptation, self-repair (see Chapter 3), and reproduction are all emergent properties (Chapter 4) of the laws of physics that have evolved within the biological world. If we look closely at biological nano-machines, such as molecular motors, they appear to resemble their human engineered counterparts. However, the way they work is often dramatically different. In most cases, the biological mechanisms hinge on physical behaviors existing only at the nanoscale.

At the present time, many functionalities of living systems exceed those of most comparable human engineered technologies by so great a margin that, if it were not for the a priori existence of life, they might be inconceivable. But living systems do exist; life thus provides the proof-of-concept for what can physically be achieved with nanotechnology. Biological nanotechnology further offers a great wealth of design concepts and strategies to achieve functionalities that would be of great utility for numerous technological goals. To take two examples, consider the ease with which living systems transform and store energy or their abilities to perform self-repair and adaptation. The ways in which energy, entropy, and information are manipulated within living nanosystems provide us with lessons on what humans must learn to do in order to develop similarly sophisticated technologies.

Implementation and utilization of complex nanotechnologies with capabilities approaching those found within the biological world is quite beyond reach at present. This chapter focuses on the specific issues associated with bringing this level of technology under human control. There are two fundamentally different ways to approach these challenges. One approach follows a long-standing human tradition of domesticating living systems and directing their capabilities to our purposes. Can this top-down approach be extended to the molecular level to tap the existing world of biological nanotechnology by constructing molecular-level, functional interfaces between living systems and synthetic soft-matter and solid-state technology? The other strategy is bottom-up, aiming to develop design and fabrication principles that enable the construction of synthetic devices, with functionalities approaching those of living systems, but with different hardware implementation.

Our consideration of these concepts is divided into four sections. The first explores the challenges of interfacing biological and non-biological systems. Communication is a major theme, as we must learn the chemical language of cells in order to convey information to them, and ultimately domesticate them. Materials fabrication and self-assembly also emerge as important components here because to interface with living systems, we must also be able to handle their hardware configurations. In the following section, this theme of interfacing is broadened to examine more generally the challenges associated with functionally connecting the nanoscopic and macroscopic worlds. In the third section, the discussion moves towards challenges of controlling energy and information on the nanometer scale. We examine the fundamental differences between the nano- and macroscale worlds, such as the importance of fluctuations, and how existing biological nanotechnology has evolved to embrace and 
utilize these features to productive ends. In the final section, challenges associated with the realistic fabrication and implementation of nanoscale devices are addressed.

\section{INTERFACING BIOLOGICAL AND NON-BIOLOGICAL SYSTEMS}

Living systems may well comprise the most complex collection of phenomena encountered in the physical world. A living cell is highly organized on every lengthscale, from the molecular to the macroscopic (Figure 5.1). Biological sciences have made astounding progress revealing this natural nanotechnology over the last 50 years. Beginning with the original clarification of the molecular structure of DNA, we have now reached a point where a nearly complete molecular parts list of a living cell is in hand. While achieving a thorough

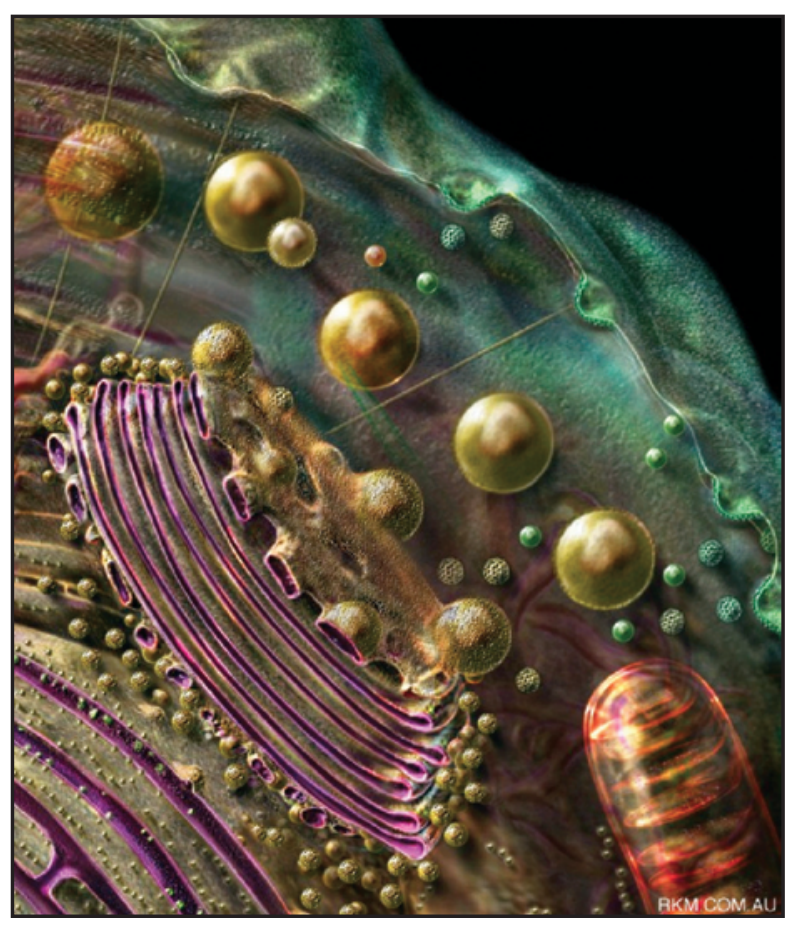

FIGURE 5.1. Artistic rendition of a living cell illustrating nanometer-scale structures. Images such as this are based on extensive electron microscopy imaging data and are quite realistic. The region illustrated is about $1 \mu \mathrm{m}$ across. The visible structures are organelles comprised of lipid membranes studded with a vast array of different membrane proteins. The cell is also filled with extremely high concentrations of soluble proteins and polymerized protein structures (e.g., cytoskeleton); these are not drawn in the image. understanding of the functionality of these systems lies (for the moment) squarely in the category of science fiction, there is a very real possibility of using the molecular knowledge we have of living systems to build functional interfaces between the living and nonliving. In this way, it may be possible to harness the unmatched capabilities of living systems in areas such as chemical, energy, and information transduction, and apply them to our own purposes within integrated devices or architectures.

\section{Actively Communicating with Cells and Directing Cellular Behavior}

The key to domesticating life at the cellular and molecular level is communication. Living cells have tremendous ability to follow directions and perform functions on demand. One need only look at any multicellular organism, from something as simple as the algae that self-assemble to form kelp to structures as complex as human beings, to see the dramatic effects of cells altering their behavior in response to input signals. It does not take a trained biologist to recognize the difference between a neuron from the brain and a liver cell. These two cell types perform extraordinarily different functions, yet they have identical DNA and are ultimately derived from the same embryonic cell. They differ as a result of input signals they have received during development. So if we were able to learn this chemical language by which cells communicate and use it to send our own signals to cells, it ought to be possible to elicit the full range of biological functions on demand (Figure 5.2).

In order to effectively communicate our intentions to cells, we must be able to produce synthetic materials that present the signals necessary to obtain technologically useful behaviors. To integrate a cell into a device will require the simultaneous presentation and modulation of multiple signals, perhaps with controlled spatial and temporal juxtaposition as well. A series of remarkably successful, albeit nascent, experimental advances addressing these issues has emerged over the last decade. Cellular behaviors previously thought to occur only within a living organism have been elicited in hybrid live cell-synthetic surface configurations. One such system, involving formation of a signaling junction between a 


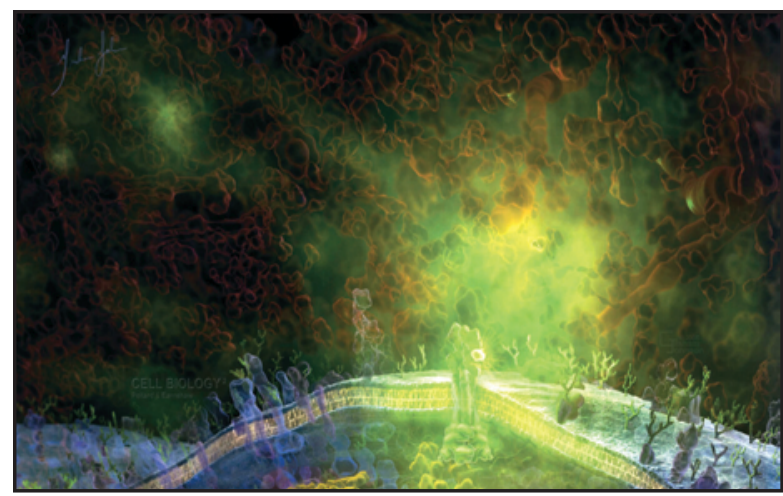

FIGURE 5.2. Artistic rendition of the initiation of a signal transduction event by ligand binding on the cell surface. The illustration of the lipid bilayer structure of the cell membrane and (in the lower portion of image) individual membrane-spanning receptor proteins is based on known structural information about the cell membrane and various proteins. A signaling molecule is also shown (bright green, lower center) binding to its ligand-binding site on a receptor protein. This composite illustration is imaginary, as there are no imaging techniques yet available that can reveal the dynamical structure of a living cell at this level of detail. To be able to do so would be extremely beneficial to efforts to harness biological nanotechnology.

T cell (from the mammalian immune system) and an inorganic surface that has been derivatized with a synthetic cell membrane and relevant signaling molecules is described in the Sidebar "Communicating with Cells."

The concept of interfacing cells with large-scale synthetic materials and devices is just one strategy. It is also conceivable that nanometer-scale synthetic devices could be implanted within cells to control and enhance cellular function. Regardless of which configuration is employed, it will be essential to have a molecular-level understanding of cellular operations as well as sophisticated synthetic capabilities to directly interface biolgical and non-biological systems.

The depth of biological complexity is difficult to perceive. While successes connecting cells and synthetic surfaces have been tantalizing, it is essential that we not lose perspective on the magnitude of the challenge ahead. To date, only a handful of signaling molecules have ever been used in a synthetic setting. The full vocabulary of cells includes thousands of molecular "words," not to mention all the various ways they may be composed into "sentences" and "paragraphs." It is clear that no linear extrapolation of scientific development as it has proceeded thus far will get us to this level.
A major challenge then is to take the vast quantity of detailed biochemical information that is becoming available and learn how to use it within a synthetic setting. This will require development of scalable strategies for the production of synthetic materials displaying biological signals, as well as screening strategies that will allow the examination of an immense set of combinatorial possibilities. This transition can be compared to the transition from pencil and paper computation of 50 years ago to the sophisticated high-performance computations of today. Very large system-integrated experimental platforms, such as are beginning to be applied to the gathering of biochemical information, will need to be applied to the synthetic implementation of these molecular systems. Significant development of soft-matter fabrication technology will be essential. Living cells themselves could also be significantly reengineered by molecular-biology techniques to be better adapted to device integration.

Just as important as being able to send signals to living cells is the ability to read out the internal state of a cell. At the ultimate level, this requires determining the identity and location of all molecules in the cell. In addition, we must have the capability of interpreting this physical configuration and relating it to the corresponding functional state.

From a practical perspective, great advances in nanometer-scale imaging and analytical technology are needed. In particular, extending (far-field) optical fluorescence (and other spectroscopic) microscopies to the nanometer scale is key. Scanning-probe microscopies can also make pivotal contributions to this effort, if they can be extended to allow molecular-scale imaging in living cells. Despite several intriguing successes in highly specialized cases, nanometer-scale imaging within living cells is quite out of reach today.

\section{Developing Minimal Self-Sustaining (Living or Nonliving) Organisms}

Creating a minimal self-sustaining system would be immensely illuminating from an intellectual perspective. By stripping away as much complexity as possible, it will become progressively easier for humans to understand the totality of the functioning system. Just how complex such a minimal system would be is 


\section{COMMUNICATING WITH CELLS}

The cell surface is studded with thousands of receptor molecules that bind to specific signal molecules. Cell surface receptors detect the presence of their cognate ligand (signal molecule) and transduce this information to the interior of the cell. At this point, a signal-transduction system, which consists of a complex series of chemical reactions, is triggered. Signal transduction systems evaluate the strength of a signal and can perform all sorts of logical operations on it. These can include simple things, such as computing time derivatives of temporally varying signals, to complex comparisons among multiple input signals. The final result is a specific shift in which genes are translated into proteins from the cell's DNA. As these newly produced proteins assume their function in the cell, a new mode of behavior is initiated.

In order to communicate with cells, the relevant signaling molecules must be presented in the right way to convey a meaningful signal. One system where molecular-level communications between a synthetic surface and a living cell has been achieved with remarkable success involves $T$ cells from the mammalian immune system. $T$ cells communicate with their target cells by forming a physical junction within which signaling molecules and receptors on both cell surfaces are able to interact. Over the last several years, inorganic substrates that have been coated with supported lipid membranes containing the relevant signaling molecules for the cell surface have been successfully used as synthetic partner cells for living T cells (Figure 1). Amazingly, $\mathrm{T}$ cells respond to these synthetic surfaces in much the same way as they respond to other living cells. Thus, in this case, the right signaling molecules, presented in the right configuration, convey a very specific message to trigger the $\mathrm{T}$ cell.

An interesting feature of this system is that signaling molecules on the synthetic surface must be able to move over large distances in order to functionally convey the signal. This requirement was not anticipated, so that for many years scientists have been presenting signaling molecules to cells in static configurations, with far more limited success. The critical advance was use of a supported membrane. This structure consists of a lipid bilayer with the same core structure as a living cell membrane that has been assembled onto an inorganic oxide surface. Membranes in this configuration can be tightly associated with the solid substrate, but still retain lateral fluidity. Lipids and signaling molecules in the membrane are free to move about on the surface, but they do not leave the membrane. This mobility allows the signaling proteins, along with their corresponding receptors on the live $\mathrm{T}$ cell, to assemble into functional signaling complexes. Only after this happens is the signal triggered.

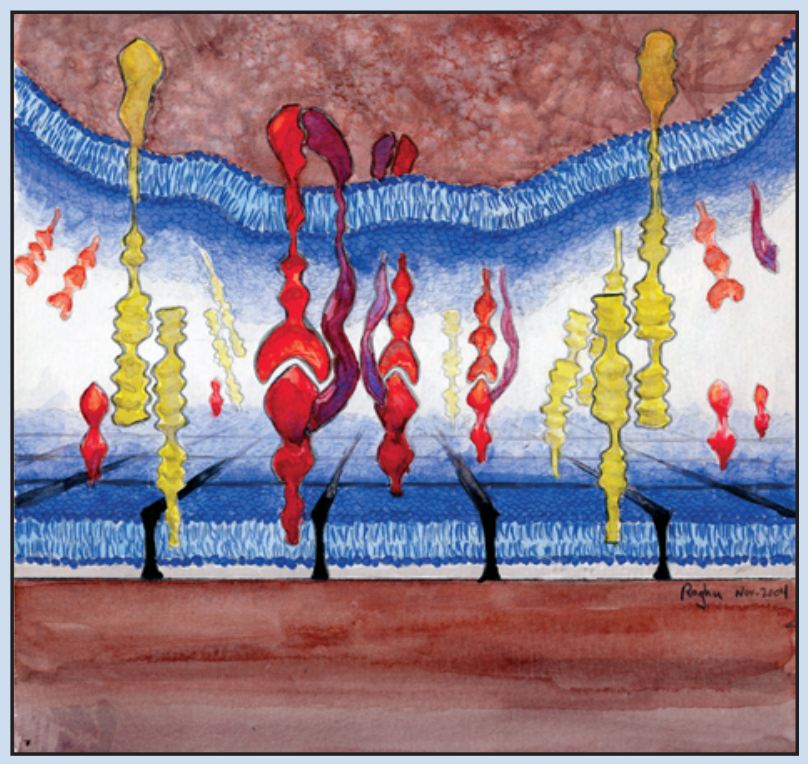

FIGURE 1. Artistic rendition of a system where molecular-level communications between a synthetic surface and a living cell has been achieved. Inorganic substrates that have been coated with supported lipid membranes containing the relevant signaling molecules for the cell surface have been successfully used as synthetic partner cells for living $T$ cells. 
unknown. To learn this would be a great step towards understanding how life may have originated. More practically, it will also point the way towards designing the simplest possible solution, to produce devices that integrate strategies from the biological world among their functioning elements.

Much of the biochemical revolution of the past halfcentury has been fueled by the ability to molecularly reengineer living cells. The now classical technology of molecular biology, by which a cell's DNA is directly manipulated and rewritten, continues to be the dominant experimental technique by which biology advances. A molecular-biological approach to minimizing a living system is a natural way to try to develop a minimal cell. Large-scale genetic reengineering efforts have already led to significantly altered forms of life. For example, cells with an altered genetic code enabling them to incorporate unnatural amino acids into their proteins have been developed. Entire metabolic pathways have also been implanted in unnatural host cells. These experimental feats have made the cells more complicated than they were naturally, but their success illustrates that large-scale genomic manipulation is a viable strategy.

A bottom-up strategy to development of a minimal self-sustaining organism is also a possibility. Certainly there are biological processes whose complexity we can't even perceive, let alone design. On the other hand, many complex biological processes have been exported out of living cells and can be performed in vitro. Synthesis of proteins from genes is a notable example in which the machinery for reading the genetic code and synthesizing the corresponding proteins has been purified and reconstituted. Perhaps other elaborate functions, such as self-repair of the photosynthetic machinery, could also be achieved in vitro. If several such systems could then be combined into self-assembling lipid vesicles, for example, it may be possible that some type of self-sustaining device could be synthesized. From the biological world we see a number of complicated tasks accomplished with brilliantly simple design solutions. Thus, even as we dig into extremely complex biological systems, the possibility of discovering simple and usable strategies is real.

\section{NANO-MACRO JUNCTIONS}

The previous section emphasized that, to understand and control biological systems, we need facile interfaces between the nano world and the macro one. This is a daunting task: humans live at the meter scale, while the atoms and molecules of which we are composed exist at the nanometer scale. A factor of a billion separates these two worlds-as big a gap as exists between our size and the distance to the moon. During the last 50 years, scientists and engineers have bridged much of this gap between the nano and the macro. A challenge for science in the $21^{\text {st }}$ century is to finish the job, making possible the ready flow of information and energy between the human and the molecular world.

Already, great strides have been made. The miniaturization of electronics has extended the reach of human engineering to less than $100 \mathrm{~nm}$, and imaging techniques can yield increasingly accurate pictures at this scale. Coming from the other end of the size scale, chemistry is learning to build ever-more complex structures from atomic and molecular precursors (as discussed in Chapter 3). What remains, however, is a formidable gap, roughly a factor of 1000 in size, where only the most rudimentary tools exist to bridge the nano and the macro worlds. Covering this last gapfrom a few tenths to a few hundred nanometers-is critical to nearly all of the Grand Challenges proposed in this document. This size range, where quantum meets classical, molecular meets bulk material, and fluctuations dominate, also challenges us to deepen our understanding of the fundamental workings of nature at all scales. There are many ways to connect the nano to the macro, from wires that deliver electric and/or magnetic signals to physical probes that poke with atomically sharp tips. An example is shown in Figure 5.3, where a single molecule - a carbon nanotube-is connected to electrical contacts. These contacts inject electrons and holes that recombine in the nanotube to emit light, creating one of the world's smallest LEDs. Below we discuss the broad classes of nano-macro junctions and indicate the promises and challenges with each. 


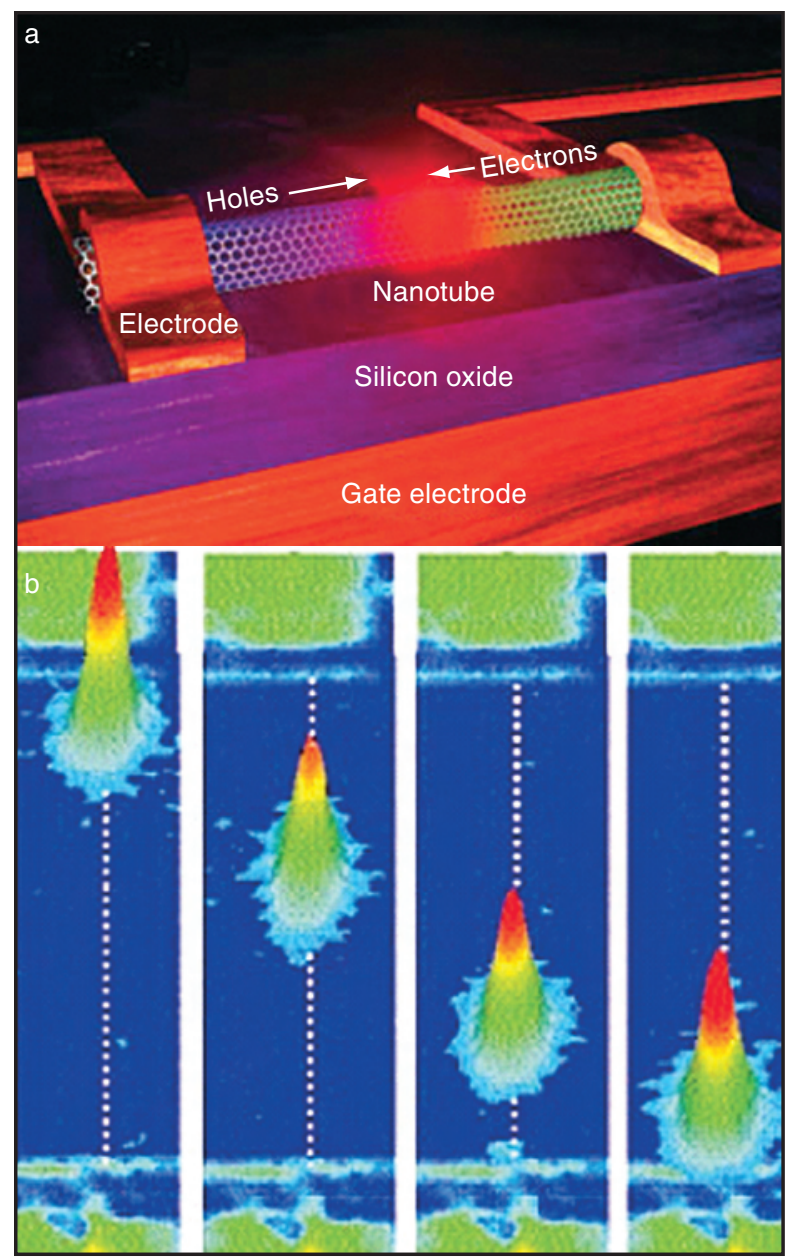

FIGURE 5.3. Top: Schematic of a nanometer-sized p-n junction created in a carbon nanotube. Bottom: Light emission as the $p-n$ junction is moved along the length of the nanotube demonstrates a tunable, nanometer-scale light source.

\section{Photonic}

Electromagnetic radiation (quantized into photons) is one of the most ubiquitous and powerful probes of the properties of matter. For the nanoworld, however, there is an obvious limitation: The spatial resolution achievable with photons is essentially determined by the photon wavelength. For visible radiation, this is approximately $500 \mathrm{~nm}$, too large to directly probe the nanoworld. There are, however, at least three ways to circumvent this resolution limit:

Use shorter wavelengths. This has been the (largely successful) approach of the electronics industry in pushing the limits of lithography. The challenge here is to create sources, lenses, etc., that work into the deep$\mathrm{UV}$ and $\mathrm{x}$-ray range. A problem, however, is that these high-energy photons can readily damage materials by causing bond breaking and ionization.

Confine the light. Light of a given frequency can be channeled into smaller spaces than a wavelength by using dielectrics and metallic waveguides. The emerging field of plasmonics, where the collective excitations of electrons guide light, promises to push visible optics to the nanometer scale.

Use nanometer-scale sources and absorbers. An atom can emit and absorb visible light, even though it is more than a thousand times smaller than the wavelength. Furthermore, its efficiency as an emitter and/or absorber can be controlled by small changes in its environment. It may therefore be a molecular-scale reporter that merely uses visible light as the route of communication. For example, gold nanoparticles shift color when they are in close proximity, and this color shift can serve as a molecular ruler to measure the distance between the nanoparticles. If these particles are tethered to a protein, for example, the functional geometry of the protein can be monitored by the color of the nanoparticle assembly.

All of these techniques will be important in the coming years. To fully push forward, we must both improve our understanding of the interaction between light and matter, as well as develop new technologies to manipulate, confine, and detect light.

\section{Electrical and Magnetic}

The miniaturization of electronics was the first engine driving the nano revolution. The feature size in the state-of-the-art integrated circuit is now below 100 $\mathrm{nm}$ and getting smaller (see the Sidebar "Defectology and the End of Moore's Law").

Fabrication of electronic devices below this scale, either to create smaller circuitry or to wire up individual molecules, is an enormous challenge. The potential payoffs are comparably huge, from extending Moore's Law (see Chapter 2) to realizing entirely new functionalities by exploiting single molecules for computation and sensing. Design parameters and stability raise major issues to be confronted with such devices. For example, electrical resistance is notoriously sensitive at the molecular scale: the resistance of a device can 


\section{DEFECTOLOGY AND THE END OF MOORE'S LAW}

The primary technology driver for the information technology industry has been Moore's prediction that the number of transistors on a chip will continue to double every 18 months. In concert with this exponential growth in transistors has come the dramatic increase in performance of integrated circuits, while the cost of a single chip has remained fairly constant. This astounding improvement over decades has had a major impact on society.

The end of Moore's scaling law has been a long time coming, but now we are quickly running up against a very fundamental obstacle, the granularity of matter. We will not be able to build device components with sizes that are a fraction of a single atom. Thus, we know that there is an end to "traditional" scaling of transistor sizes, but we can't predict exactly when that will occur. This is because it is not just a physics or engineering issue; it is also an economic question (Figure 1).

Today's logic chips are perfect because they have to be: Just one broken component means the entire processing unit is broken. However, as the feature sizes of devices scale down to the few-nanometer scale, there will be inevitable statistical fluctuations in the number of atoms in a component of a transistor or a wire. If the fluctuations are severe enough, the device won't work at all, which means the entire circuit is broken.

Devices in a circuit may be nonfunctional because of manufacturing errors, which we call defects; or they may yield incorrect results because of a fluctuation in a prop-

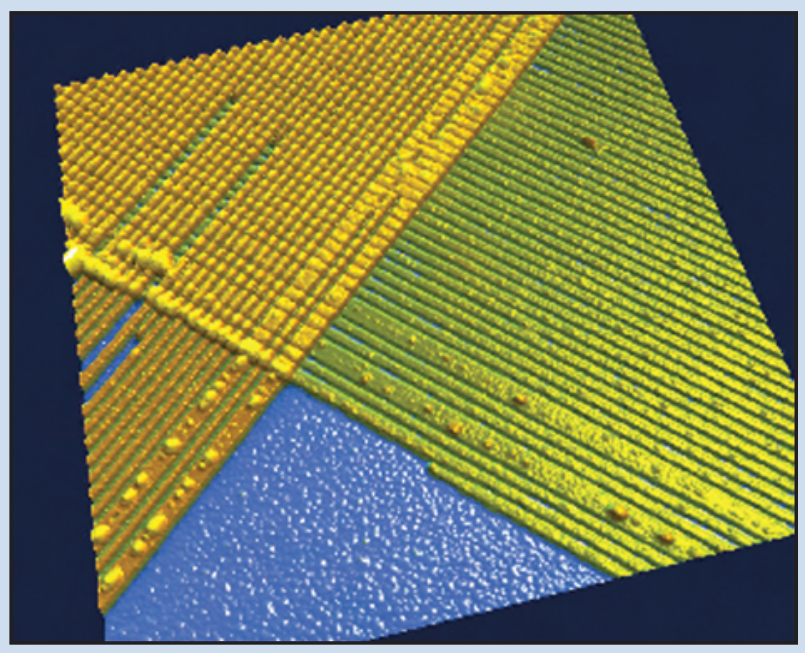

FIGURE 1. An atomic force microscope topograph of a crossbar circuit fabricated by imprint lithography at a feature size (halfpitch) of $17 \mathrm{~nm}$. The atomic-scale granularity of matter is evident in this image because of the "bumpiness" of the surface image. Also, at this scale, various types of defects are obvious in the image, e.g., broken wires and filled-in gaps between wires. At some scale, it will no longer be economically sensible to build such structures perfectly. erty during operation of the circuit, which we call faults; or devices that start out working properly but then experience a catastrophic event, which we call device death. The challenges for integrated circuits with billions or trillions of nanoscale components are that the probability of a problem with an individual component in a circuit is increasing dramatically with decreasing size. We will very quickly cross the threshold from high-yield circuits that perform reliably for long periods to low-yield circuits that experience frequent interruptions and device deaths.

The fundamental architecture of a chip in the future will have to incorporate defect, fault, and death tolerance. This question of building reliable machines out of unreliable parts was a significant area of research in the pioneering days of computing in the 1950's and 60's. It is only now that we are entering into the nanoscale age that we need to seriously reexamine the issues of how to build reliable machines given that they will be manufactured with defects and experience faults and device deaths.

A major approach to making a logic circuit reliable and resilient in the presence of defects, faults, and deaths deals with optimization of redundancy of circuit elements. A small amount of redundancy can provide an exponential increase in the reliability of a circuit, which is excellent in terms of keeping the cost of the error correction to a minimum. Another approach to building more robust circuits is to use coding theory to design and build redundant circuits that contain efficient automatic correction for errors of various sorts. Such an approach is effective for defects, faults and deaths, although it is limited in the types of functions that can be protected. However, at this stage it does not appear 
possible to use coding theory to efficiently protect general Boolean logic circuits against unlocated device defects and deaths. It is conceivable, although not at all certain, that by combining reconfiguration and coding one may be able to construct extremely resilient systems that defend against all error types. This is an active field of research.

There are also opportunities for alternative computational paradigms, such as "neuromorphic" or probabilistic, which are inherently more robust to device defects, faults and deaths. Such approaches generally use simple, regular structures (such as crossbars) in vast numbers that are easily fabricated at the nanoscale (using, for example, self-assembly) and acquire their functionality through self-organization and training rather than detailed design. Although such schemes are generally poor at attacking the number-crunching problems of traditional, logic-based computers, they have the potential to excel in problems that today's computers are notoriously bad at: pattern recognition and adaptive interaction with the environment. As such, these might provide an important complement to our existing computational infrastructure.

change by an order of magnitude if only a single atom moves. Since atomic motions cannot be stopped at ambient temperatures, a functional device must be somehow able to tolerate large-scale fluctuations in its hardware configuration. Biological nanoscale devices face this same problem and have a variety of interesting strategies for its successful management, as will be discussed further below.

While there have been successes is molecular electronics (See Sidebar "Molecular Transport Junctions" and the discussion in Chapter 6), to date there is no approach with the sophistication and reproducibility that is needed. One promising direction is to construct a generic interconnect that can reliably bridge between 100-nm-scale metal electrodes and specific chemical end groups of molecules. A number of candidates exist for this molecular-macro interconnect, such as functionalized carbon nanotubes. The challenge is to wire complex molecular circuits with a large number of interconnects, so that multiple currents or voltages can be applied to a molecular complex only nanometers in size. We are still far from addressing this challenge.

Magnetism is the partner to electricity, and magnetic storage is a key component of modern information technology. Furthermore, the magnetic properties of atoms can be remarkably powerful probes of molecular identity and local structure. For example, magnetic resonance imaging (MRI) routinely yields detailed images at the millimeter scale. This concept can be pushed to the nanometer scale in the highly specialized magnetic resonance force microscopy (MFRM) configuration. Nuclear magnetic resonance (NMR) spectroscopic techniques can be used to determine protein structure, though ensembles of identical molecules are now required. The major challenge to further miniaturization of magnetic technologies is predominantly one of signal size. Magnetic interactions are weak, and only very specialized techniques (e.g. MFRM) can detect the magnetic signature of a small number of spins. The weak signal is an important limitation for information technology, but even more so for using techniques like MRI to image at the nanoscale. Techniques that extend spin detection and imaging below $100 \mathrm{~nm}$ will be important for the further evolution of information and magnetic-imaging technology. A true breakthrough would allow routine MRI-type imaging and spin manipulation at the molecular scale.

\section{Mechanical}

Physical interaction between the nano and macro worlds is perhaps the most intuitive and direct interface that we can envision. To touch, tug, and arrange at the single-molecule scale is a dream of both the scientist and the technologist. Scanned-probe techniques such as scanning tunneling microscopy (STM) and atomic force microscopy (AFM) have already made enormous strides, but the level of sophistication is more or less comparable to a blind person with only one finger. Optical tweezers, in which forces resulting from a tightly focused laser beam are used to manipu- 


\section{MOLECULAR TRANSPORT JUNCTIONS}
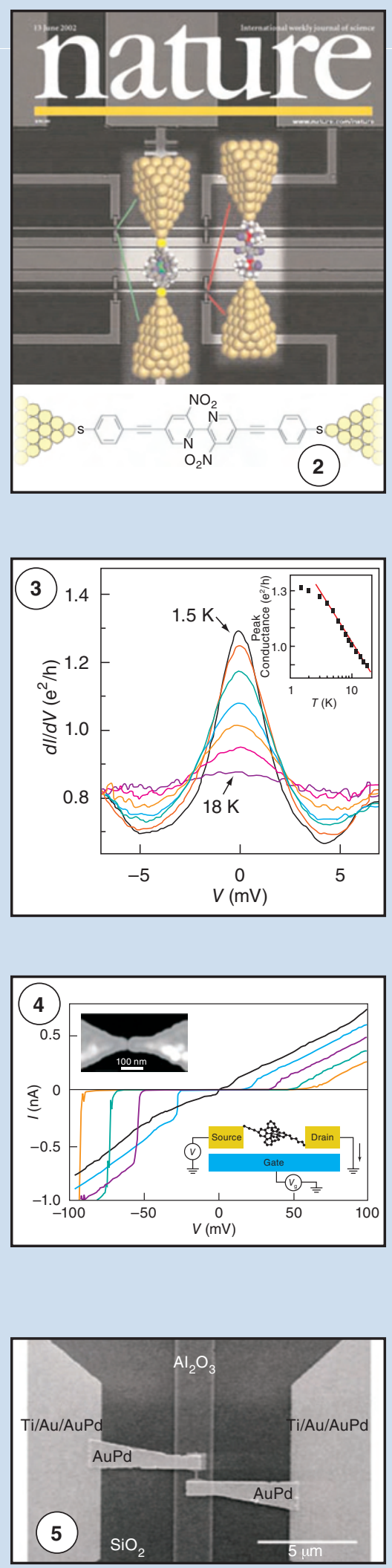

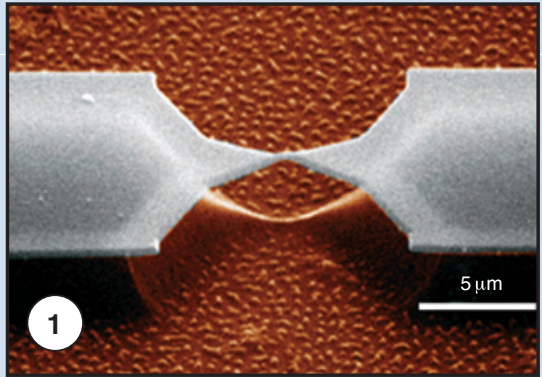

Understanding charge motion in molecular transport junctions is just beginning. Below some of the simplest examples of single-molecule nonequilibrium behavior are illustrated in sketches and images of junctions, in which single molecules, such as the complex molecules shown on the Nature cover, act as conductors in a highly non-equilibrium situation:

- (1) Image of a fabricated metallic break junction. A very narrow contact is stretched by flexing the support until a single atom makes the contact.

- (2) Sketch of molecular junction with an organic $\pi$ system suspended between two metallic electrodes.

- (3) The Kondo resonance, where the conductance through the junction can become very large at low temperatures (see inset), is a characteristic phenomenon due to strong correlations of the electronic system (see Chapter 4).

- (4) In the so-called Coulomb blockade (also a characteristic phenomenon due to strong correlations of the electronic system), depending on the nature of the gating voltage, there can be a long flat area around zero source voltage, when the electron simply cannot be forced onto the molecule, so no current is observed. Remember that if this structure followed Ohm's law, the graph would show a simple straight line sloping upward from origin.

- (5) A molecule rests between the two silver-palladium pads suspended above the alumina gate in the middle of the picture.

- (6) The characteristic Coulomb-diamond structure of the molecule's conductance dependence on a source voltage $\left(\mathrm{V}_{\mathrm{G}}\right)$ in (5).

- (7) The current-temperature behavior at different source voltages of the molecule in (5).
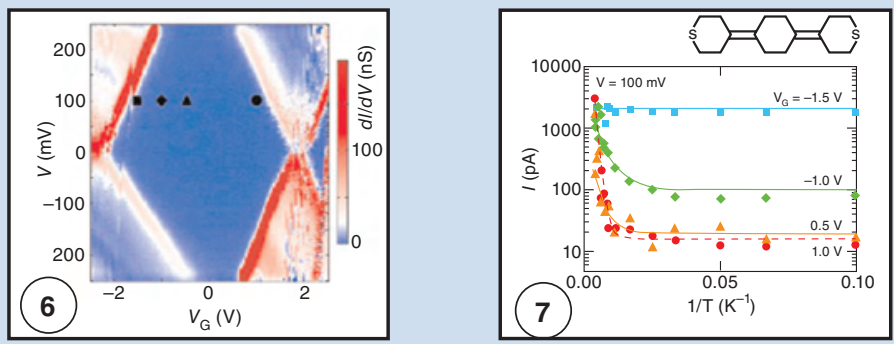
late matter, possess similar limitations. The difficulties in creating the next generation of physical interaction tools are coupled to the other nano-macro problems discussed above. As with the nanoelectronic case, we must seamlessly connect macro-scale manipulation systems to well defined functional nanoscale probes. These probes will be sensors and manipulators, using forces, photons, and electric and magnetic fields to both feel and push at the nanoscale. Also required is real-time nanoscale imaging to see what is happening.

\section{Combining Different Approaches}

The task of creating nano-macro interfaces is one that requires simultaneously integrating more than one approach. In the past, these different fields have evolved more or less independently. Researchers working on molecular wiring were not also working on plasmonics, for example. This isolation is changing, and the change needs to be accelerated. No one technique will provide all that is needed to build a successful interface between the nano and the macro. Each helps the other: imaging helps physical assembly, which can subsequently improve electronics, etc. The positive feedback loops that can occur between different techniques should be fully exploited. To do this requires encouraging work that crosses disciplinary lines (as addressed in Chapter 7).

\section{ENERGY TRANSDUCTION AT THE NANOSCALE}

At the nanoscale, the fundamental quanta of energy (e.g., photon, electron, phonon, etc.) become important. The study of quantized energy flow in molecular systems has been the mainstay of physical chemistry for decades, and great progress has been made in using structure to tune these properties. In molecules, the individual atomic nuclei are relatively few and the electronic orbitals that lead to chemical bonding are quite discrete; that is, their properties are determined by a relatively small set of nearby atomic nuclei. Nanoscale systems typically contain thousands or billions of atoms, which may be coupled to yield a broad continuum of collective states. As a result, there is a degree of adjustability in the design of nanoscale-system properties that differs greatly from smaller molecular systems. Learning to use this design flexibility to control the flow of energy down to the individual quanta represents a challenge that could lead to a wide variety of new technological capabilities.

\section{Photonic, Electronic, and Chemical Transitions}

Photon-electron coupling. One of the most technologically important processes is the conversion of light energy to electrical energy. The classic semiconductor device for accomplishing this is the $\mathrm{p}-\mathrm{n}$ junction: photons are absorbed in a semiconductor, creating electron-hole pairs that are separated by the built-in electric field of the junction. These are "harvested" by an electric circuit and can be stored in a battery. The solar-cell industry is based on this device. Breakthroughs here could have billion-dollar implications and could have even more significant impact on the global energy economy. While high photoelectric energy-conversion efficiency has been achieved in certain exotic materials, no globally scaleable technology is available that operates at high efficiency. Learning how to control photon-electron energy conversion using common elements and easily manufactured materials is a major challenge that requires a deeper understanding of energy flow on the nanometer scale.

Solar cells are just one example of the important science that needs to be explored in electron-photon energy conversion. The inverse process is also important- the conversion of electrical energy into light. Lighting comprises a significant fraction of total electricity usage in this country, but it is also highly inefficient, with incandescent bulbs converting just 5\% of electrical energy into light. The challenge here is to engineer devices that can produce light with efficiencies approaching $100 \%$. The simplest device geometry is the light-emitting diode, the inverse of the solar cell described in the previous paragraph. Electrons and holes injected into the device first find one another and then recombine to create photons. Making this process efficient requires a complete and thorough understanding of the injection of electrons and holes into the material, the formation of bound electron-hole pairs, or excitons, and the subsequent emission of light. While 
these processes are well-understood in simple semiconductors, the basic science remains poorly understood in complex materials, such as organics, where the electrons, the holes, and the background lattice all interact strongly. Experiments such as the one in Figure 5.3 are beginning to explore these fundamental processes in exquisite detail.

Photon-chemical coupling. Converting photon energy into a chemical fuel, e.g., the direct synthesis of alcohols or alkanes from carbon dioxide, is a true major challenge, both scientifically and in terms of potential societal impact. Scientifically, we are only beginning to understand the protein assemblies that convert sunlight into stored chemical energy in biological systems, as discussed previously in Chapter 2 and the Sidebar "Solar Fuels" in Chapter 3. It is a complex, multi-stage process, far more sophisticated than the simple solarcell process involving one photon and one electronhole pair. The challenge for nanoscience is to develop systems whose functions reach even beyond those of biological photosynthetic systems and efficiently convert sunlight into accessible chemical energy. We must integrate our understanding of electronic, vibrational, and electrochemical properties of nanoscale assemblies to predict their behavior and to design more efficient assemblies. A functional system will undoubtedly operate far from equilibrium, a dim area in our current level of scientific understanding (see Chapter 6). Technologically, we must develop fabrication strategies to create these assemblies and develop techniques to probe their properties. Nanoscale energy sources will ultimately be the engines for the self-sustaining nanoscale systems discussed in the next section.

\section{Stochastic Processes, Signals, and Noise}

The molecular systems that perform biological functions generally operate in noisy environments, where thermal fluctuations are large compared with the size of the whole system. For human-engineered devices, noise is often thought of as detrimental and systems must be designed to overcome it. For example, digital microprocessors use large voltages and energies to ensure that stochastic noise in the electronic signals does not compromise their integrity. Biological systems tend not to overpower noise in this way. Rather, evolution has embraced stochastic fluctuations and often relies on them for the functionality of the system. This suggests an interesting design principle that humans have not yet learned to use. Exploitation of statistical fluctuations may well be essential to accomplish some of the more exotic tasks living systems are able to perform (see the discussion of fluctuations in Chapter 6). Below, we will highlight two examples of biological systems in which stochastic noise is utilized in a functional manner. These illustrate some of the alternative strategies we might be able to learn from living systems. More generally, they illustrate a class of problems that are not addressed by our existing technologies, but will be essential to push forward our efforts to realize the potential of nanotechnology.

Biological signal transduction and information processing. Populations of bacteria are able to swarm and work as a group through the release and detection of molecular signals. These are sensed by chemotaxis receptors and the resulting signal is transduced, by a remarkably simple molecular logic circuit implemented with just a handful of different proteins. The output of this circuit biases the bacteria's otherwise random walk and statistically delivers the bacteria to the desired location (Figure 5.4). Though quite simple, this biological signal transduction system achieves significant behavior. An interesting aspect of its function is its reliance on stochastic fluctuations of proteins to achieve the most effective population behavior. In other words, if each bacterium in a population were truly identical, down to the exact number and assembly of each protein, then the molecular program for chemotaxis would not work very well. The fact that each cell has different numbers of proteins, owing to stochastic fluctuations, provides a robustness to the algorithm that gives it a functional advantage. Another example of the role of stochastic fluctuations in cellular signal transduction is described in the Sidebar "Noise can be Useful."

Molecular motors. The conversion of chemical energy to mechanical work is an essential aspect to numerous biological processes and could also be of great use in device engineering. Besides the obviously mechanical behaviors, such as muscle movement, many seemingly chemical processes are actually performed mechanically 


\section{NOISE CAN BE USEFUL}

\section{Immune Cells Make Definite Decisions When Dealing With Noisy Data}

Cells must often make decisions based on fluctuating (noisy) chemical concentrations in their environment. For example, the T-lymphocytes of our immune system must determine whether peptides are foreign pathogens or self peptides. It has recently been shown that dueling positive and negative feedback loops can be used by $\mathrm{T}$ cells to produce digital signals in the presence of stochastic fluctuations in the concentrations of agonist and antagonist ligands. (Agonist ligands bind to a receptor and stimulate production of signaling molecules, while antagonist ligands also bind to receptors but shut off signaling induced by agonists.) If the agonist stimulates a positive feedback loop that further provokes production of the signaling molecule, and the antagonist initiates a negative feedback loop that inhibits production of the signaling molecule, stochastic fluctuations influence the duel between the two loops in a remarkable way.

In a deterministic (mean-field) picture, each cell always produces an intermediate amount of the signaling molecule when confronted with a mixture of agonists and antagonists in its environment. In other words, the cell makes no decision because a threshold amount of signaling product is required to initiate gene transcription and the consequent immune response. In the presence of fluctuations due to small numbers of agonists and antagonists (the realworld situation), for identical conditions that lead to no decision in a deterministic world, each cell makes a clear decision-it is either "on" or "off" (see Figure 1). The proportion of cells that are "on" or "off" depends on the ratio of agonists to antagonists in the cell's environment.

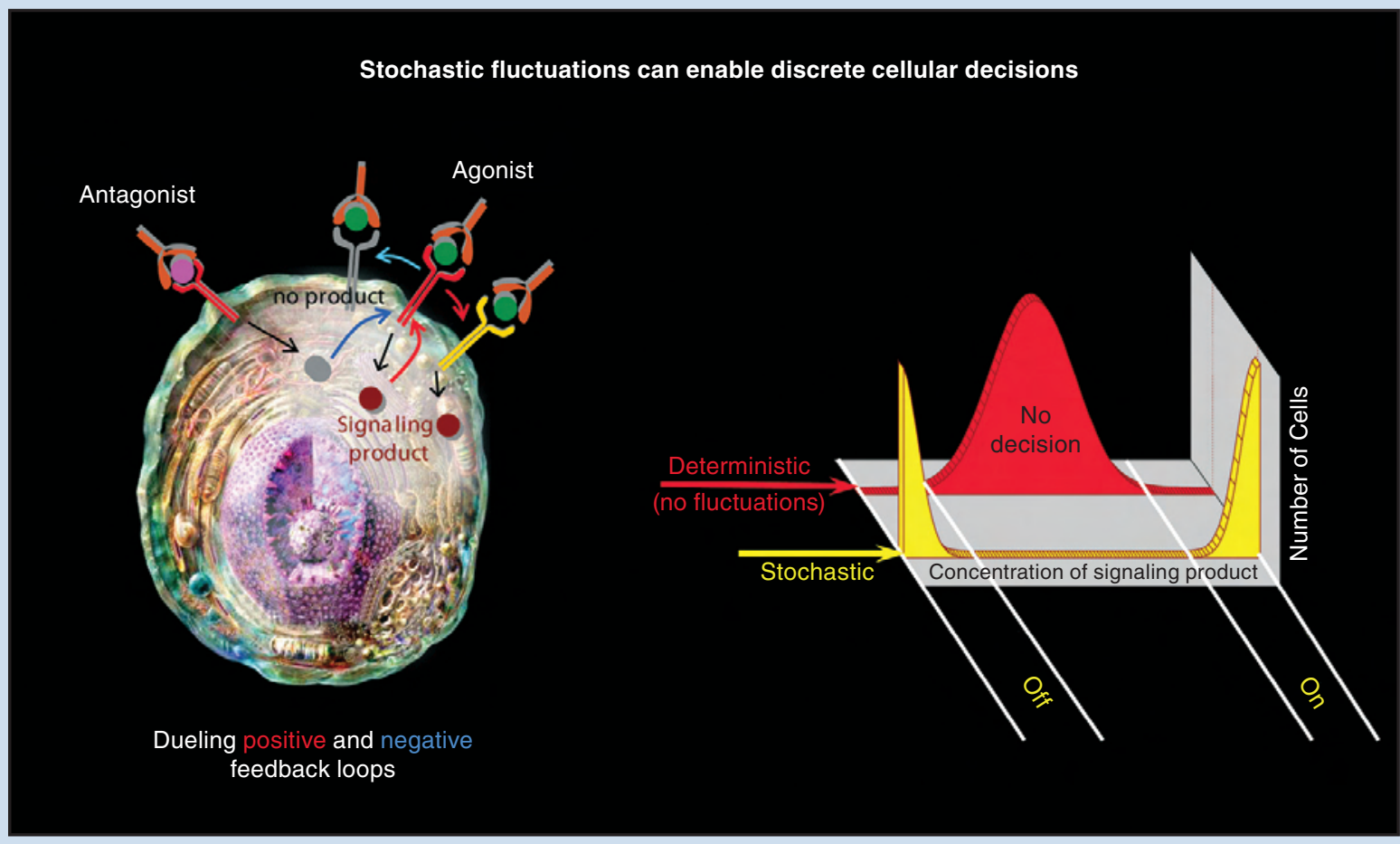

FIGURE 1. Decision making when the ratio of agonist to antagonist is about 1:1. The cells exploit stochastic fluctuations to make decisions that lead to clear functional outcomes not "confusion." This kind of digital signaling has been seen in T lympocytes. The conditions necessary for stochastic effects resulting in binary cellular decisions are ubiquitous in cell biology, and so this phenomenon may be prevalent in both natural and engineered systems. 

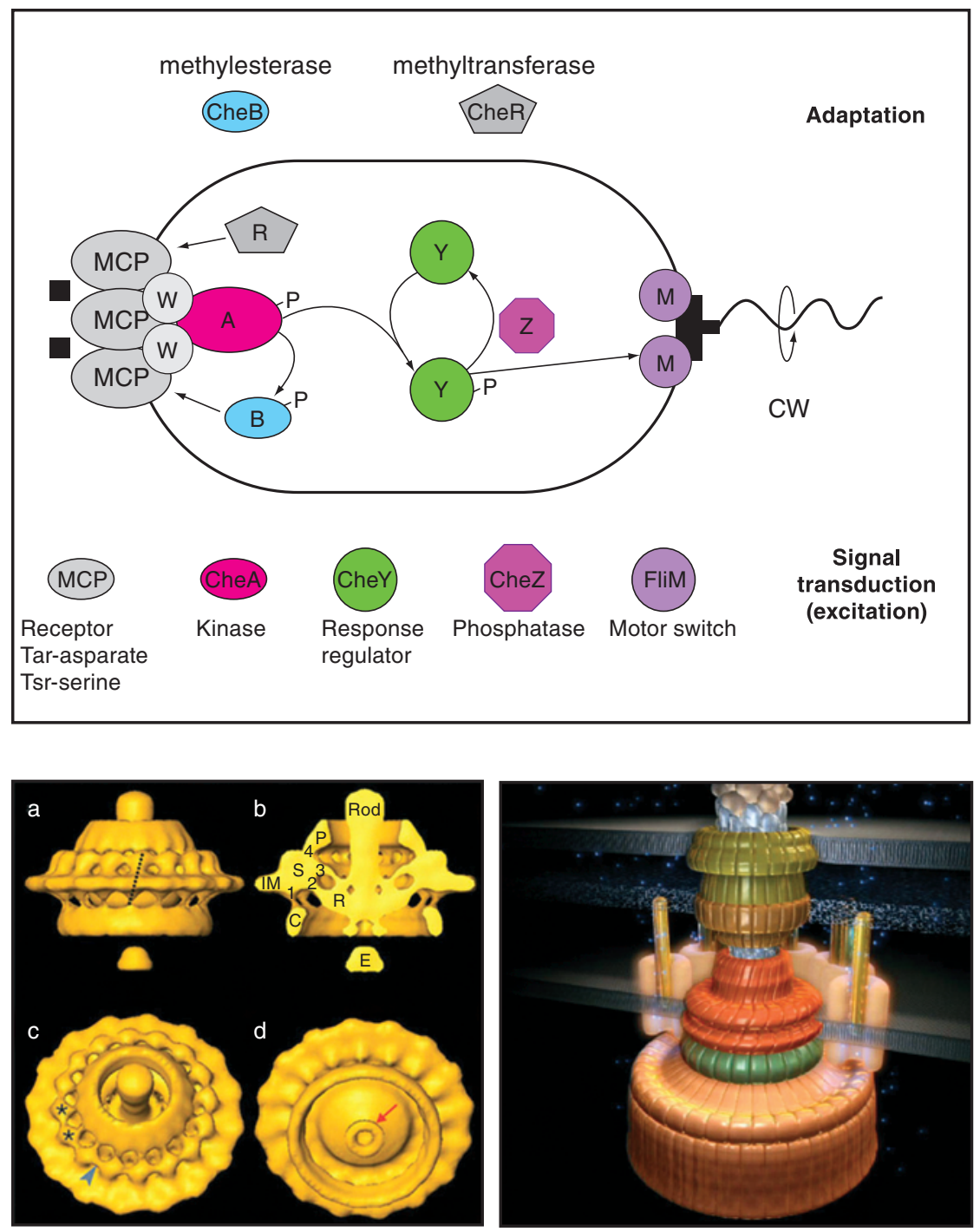

FIGURE 5.4. Schematic diagram illustrating the relatively simple network of proteins that govern bacterial chemotaxis. Signal molecules (black squares at left) bind to cell surface receptors (MCP). These receptor proteins transduce information about the binding event to other proteins in the cell interior ( $W$ and $A)$. These, in turn, modulate the activity of other proteins ( $B$ and $Y$ ), which then modulate the activity of MCP and the flagellar motor (M), respectively. This network of a half-dozen signaling proteins computes temporal derivatives of the signal-molecule concentration and uses this information to direct bacterial swimming. The hardware and software are one for this simple molecular logic circuit. As far as we know, all biological molecular signal logic functions in essentially this way, albeit in many cases with much greater levels of complexity.

FIGURE 5.5. Left: Experimental threedimensional composite reconstruction of the structure of a rotary molecular motor from electron tomography imaging data. Right: Artistic rendition of a similar rotary molecular motor. These motors are several nanometers across and efficiently convert chemical energy to mechanical work. Although they may resemble macroscopic rotary motors in their appearance, their functional mechanism is quite different, as required by the different nature of physical laws at the nanometer length scale. See the Sidebar "Protein Motors." within cells. The behavior of ATP synthase is an example. This is the protein that cells use to synthesize the energy rich ATP molecule, which serves as the primary fuel for nearly all biological processes. ATP synthase is a rotary motor protein (Figure 5.5). When the motor is cranked one way, mechanical work is converted into chemical energy by adding a phosphate to the ADP molecule, thus turning it into ATP. The motor can also run the other way, burning ATP into phosphate and $\mathrm{ADP}$ and producing a torque. Molecular motors such as this generate mechanical forces by using intermolecular binding events and their associated free energy changes to capture favorable fluctuations. In the case of the rotary molecular motors, this free energy arises from the movement of ions down an electrical poten- tial. This is an extremely effective way of achieving direct chemical-to-mechanical energy conversion on the nanometer scale (see the Sidebars "Protein Motors" and "Proticity and Electricity").

\section{FUNCTIONAL NANOSCALE SYSTEMS AND COLONIES}

The small size of nanoscale devices makes it feasible to utilize large numbers of them within a very small footprint. From the fabrication perspective, large numbers of potentially highly sophisticated devices must be produced. From the functional perspective, there arises the additional issue of how such devices can coordinate 


\section{PROTEIN MOTORS}

\section{Molecular Motors Use Binding Energy to Generate Force; the Trick Is to Make a Repeating Mechano-Chemical Cycle}

Protein motors come in a bewildering variety of shapes and sizes with an equally bewildering array of cellular functions. The fundamental operation of molecular motors involves the conversion of energy, generally from chemical bonds, into mechanical work. Theoretical studies of many different motors have revealed a few simple general principles. First, binding of a ligand — usually $\mathrm{Mg}^{++}$ATP, the chemical fuel一to the motor drives a large conformational change in the region around the binding site. This deformation can directly drive a "power stroke" to deliver work to an external load, as in the $\mathrm{F}_{1}$-ATPase (see Figure 1, left, and the Sidebar "Thermodynamics of Molecular Machines" in Chapter 6) . In other motors, such as those packaging DNA into the virus capsid, the binding deformation energy is stored as elastic energy in a beta sheet structure that adjoins the ligand binding site, and this elastic energy is used to drive a "recoil" power stroke that delivers work to the translocating DNA strand. In other motors, such as myosin, the binding energy of ATP is directed towards the site where it is used to pry the motor loose from its actin track.

An essential feature of the ligand-binding-driven power stroke that distinguishes its modus operandi from macroscopic motors is that it depends in an essential way on thermal fluctuations (see Figure 1, center). The motor catalytic site has a mobile region (the "P-loop") that slides over the ATP and progressively "zips up" a hydrogen-bond network that fuses the ligand to the motor. However, this sliding is not a smooth monotonic journey; the free-energy gradient is bumpy, and were it not for thermal fluctuations to surmount the many barriers, the binding zipper could not get very far. This bumpiness on the nanometer scale results from the fundamental atomic structure of matter and applies to essentially all nanometer-scale devices, regardless of their construction or purpose.

Once the ligand binding energy has been delivered away from the binding site, the ligand is now held tightly. So how is the power cycle to be repeated? Here nature has wisely chosen $\mathrm{Mg}^{++}$ATP as the right fuel, for it contains within itself a store of energy that can be used to drive the "exhaust stroke" to rid the binding site of its ligand. This energy resides in the covalent bond that holds the terminal phosphate group to the rest of the molecule. When ATP
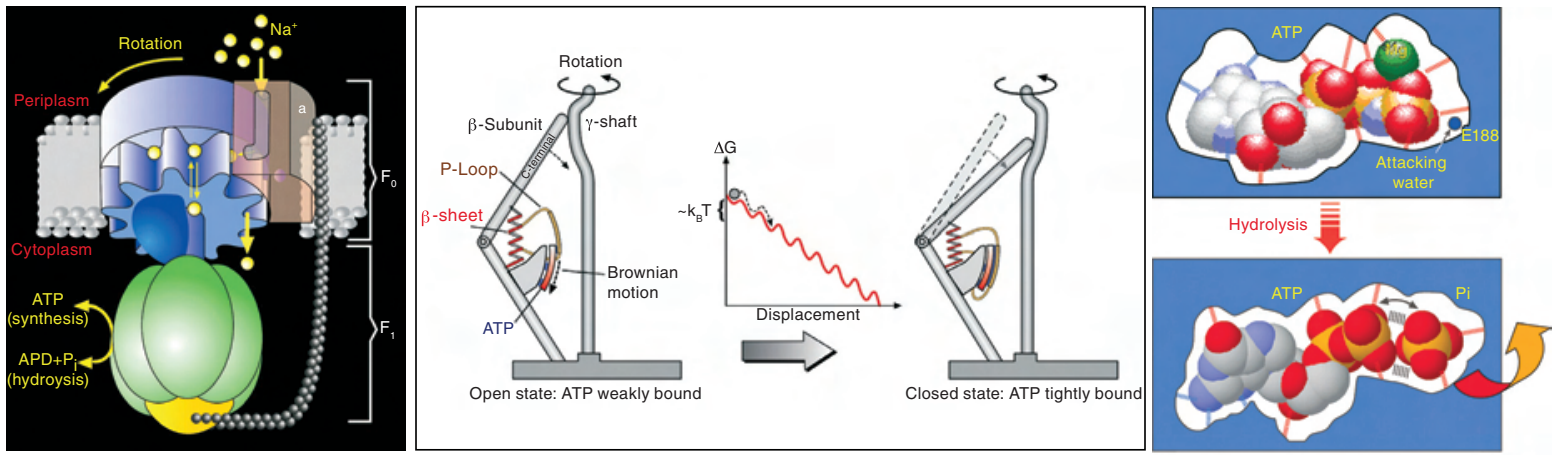

FIGURE 1. The general principles of protein motors illustrated by ATP synthase. Left: ATP synthase (or $F_{0} F_{1}$ ATPase) consists of two counter-rotating motors joined elastically. The $F_{0}$ motor is driven by a transmembrane gradient in electrochemical potential generated by the gradient in sodium ions or protons. The $F_{1}$ portion uses the torque generated by the $F_{0}$ motor to manufacture ATP from ADP and phosphate $\left(P_{i}\right)$; however, $F_{1}$ can also function as an ATPase motor, hydrolyzing ATP to rotate the $F_{0}$ motor backwards turning it into an ion pump. Center: Cartoon illustrating how the "binding zipper" generates mechanical torque. Right: Hydrolyis of the terminal covalent bond by an "inline attack" by an activated water molecule. 
has become bound to the protein, the hydrogen bond network grips it tightly in the "transition state" where it is brought into alignment with a water molecule that is held by a key amino acid residue (see Figure 1, right). This water can now capture the electron cloud that constitutes the terminal phosphate bond, thus hydrolyzing the ATP into ADP and phosphate. These two fragments are negatively charged, and so now they can repel one another strongly enough to loosen the motor's grip on them, allowing the products to be released, so that a new ATP can be processed.

There are many variations on this sequence of events, for different motors are adapted to different tasks, and protein geometry has evolved in many different directions. Nevertheless, the basic operating principles are few and easily understood as variations on the basic physical sequence of events described here.

their behavior to function in synchrony towards a desired goal. Again, we may learn from biological solutions to these problems. Can we make the equivalent of artificial cells? By this we mean a confined system of nanoscale elements with designed metabolism and communication capabilities, and perhaps even the ability to reproduce.

\section{Self-Regulating Adaptive Interactive Systems}

Without a source of usable energy any device is necessarily passive. The next stage is to create active nanoscale assemblies, ones that change and respond in controlled, well defined, and useful ways by consuming fuel. In other words, they must be metabolically active. Creating active assemblies of non-biological nanostructures that come ever closer to mimicking life in their function and adaptability is a complicated challenge. One can divide this challenge into three parts:

Metabolism. The goal here is to create a small system of interacting elements that has what biologists would call metabolism-a complex system of interacting feedback loops that takes input energy from some source, such as sunlight or chemical food, and uses it to achieve a desired objective, e.g., to produce a useful drug. A bacterium does this with ease, packing the power of a pharmaceutical laboratory in a micrometersized bag. The challenge for nanoscientists is to do the same. What this artificial "cell" will look like remains a very open question - it could be a chamber in a silicon chip fed by nanofluidic valves that allow chemicals in or out, as shown in Figure 5.6, or it may be based on a self-assembling structure. The key to establishing a non-biological metabolism is the controlled consump- tion and conversion of energy within a responsive nanoscale system.

Information replication. The second part of the challenge is equally critical. We wish to create a nanomaterial that can store information and, through a relatively simple set of steps, duplicate that information. By repeating those steps, we can duplicate it again and again to create a growing population of information-containing elements, all identical or nearly identical to the first. In life, this is done by the unzipping and reconstruction of the double-stranded molecule DNA. A fascinating challenge is to create a nano-equivalent that is not DNA. Many proposals have been made, from linear molecules like DNA but made from different building blocks, to clay microcrystallites that store information in patterns of defects. To date, no serious experimental demonstration has been achieved. Nor is it known if, perhaps, DNA is the simplest solution to this problem. The only human versions of information $\mathrm{du}^{-}$ plication require large and technologically sophisticated writing instruments, be it a hand and pencil or a hard drive. Can we make a version that requires nothing but a beaker and a few simple processing steps, e.g., heating and cooling?

System integration. Successes at the first two tasks above - creating systems with metabolism and selfreplicating information storage-would be a major accomplishment, but perhaps possible on a twenty-year horizon. Achieving these capabilities individually paves the way for a visionary challenge: integrating them into self-sustaining nonliving systems, which could be based on materials and operate in environments very different from those of living systems. 


\section{PROTICITY AND ELECTRICITY}

\section{Electricity and Proticity-The Contrast between Biological and Human-Engineered Energy Conversion}

The use of electricity as an energy source for human activity seems normal to those living in the developed world. However, biology does not use electrons flowing through wires to carry out the activities of the cell. While the underlying electrochemistry in human-engineered devices, such as fuel cells, and living cells is similar, the implementation in biology is fundamentally different. Comparing and contrasting energy conversion by fuel cells and by mitochondria illustrates the differences that must be taken into account in research that could lead to the use of elec-

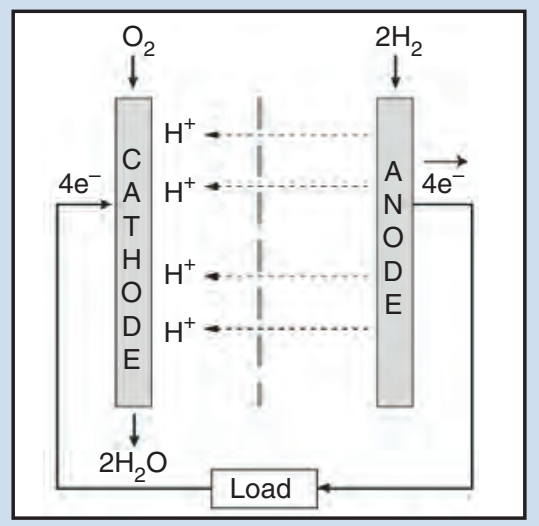

FIGURE 1. The oxidation of fuel and reduction of molecular oxygen $\left(\mathrm{O}_{2}\right)$ in a fuel cell. These processes occur at electrodes (cathode and anode) and are coupled to the generation of an electromotive force (emf).

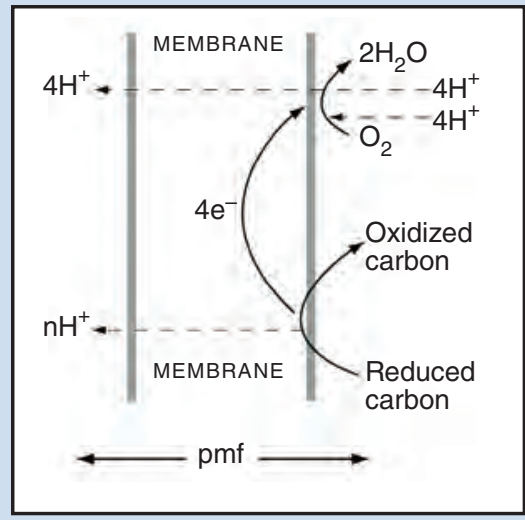

FIGURE 2. Oxidation of fuel and reduction of $\mathrm{O}_{2}$ in the mitochondrion. These processes occur at catalytic sites of proteins arranged vectorially across a membrane, resulting in an electrochemical potential of protons across a membrane, known as proticity, or when expressed as a voltage, proton motive force (pmf). tricity with biological or bio-inspired catalysts to synthesize fuel, drive biological molecular motors, or even augment the growth of organisms.

Both fuel cells and mitochondria convert the high energy content of chemical fuels to other forms of energy. In the fuel cell, the oxidation of fuel and reduction of molecular oxygen $\left(\mathrm{O}_{2}\right)$ occur at electrodes and are coupled to the generation of electromotive force (emf) in a wire (Figure 1). The wiring of the electrical circuit determines the path of the current, and a semipermeable membrane that allows ions to flow in order to maintain electrical neutrality completes the circuit.

In the mitochondrion, fuel oxidation and $\mathrm{O}_{2}$ reduction occur at catalytic sites of proteins arranged vectorially across a membrane (Figure 2). Discrete chemical species carry the electrons from site to site using the principles of molecular recognition to avoid short circuits and skipped sites. At the transmembrane sites, proton translocation across the membrane is tightly coupled to electron flow, so that electrochemical energy is translated into a concentration gradient of protons, $\Delta \mathrm{pH}$, across the membrane. Moreover, electrical neutrality at the coupling sites is not maintained, so that an electrical potential, $\Delta \Psi$, builds up across the membrane. This electrochemical potential of protons across a membrane, $\Delta \Psi+\Delta \mathrm{pH}$, is known as proticity, or, when expressed as a voltage, proton motive force (pmf).

Thus, a current of protons driven by pmf is available to molecular transducers that span energy-coupling membranes. Pmf is the common denominator underlying all bioenergetic processes of all cells. Spectacular molecular motors, such as the flagellar motor of bacteria and ATP synthase, which is ubiquitous in cells, are driven by pmf. The necessary chemical potential that drives these nanomotors is provided by either $\Delta \mathrm{pH}$ or $\Delta \Psi$. Developing constructs for emf-to-pmf conversion is central to some approaches to artificial photosynthesis and will open the door to employing biological energy-transducing catalysts coupled to pmf, in hybrid devices capable of the synthesis of energy-rich compounds. Pmf-driven motors are a paradigm for chemical-tomechanical processes in nanotechnology. Likewise, the oxygen-reducing catalyst in the mitochondrion sets the standard for efficiency and uses only earth-abundant materials. 


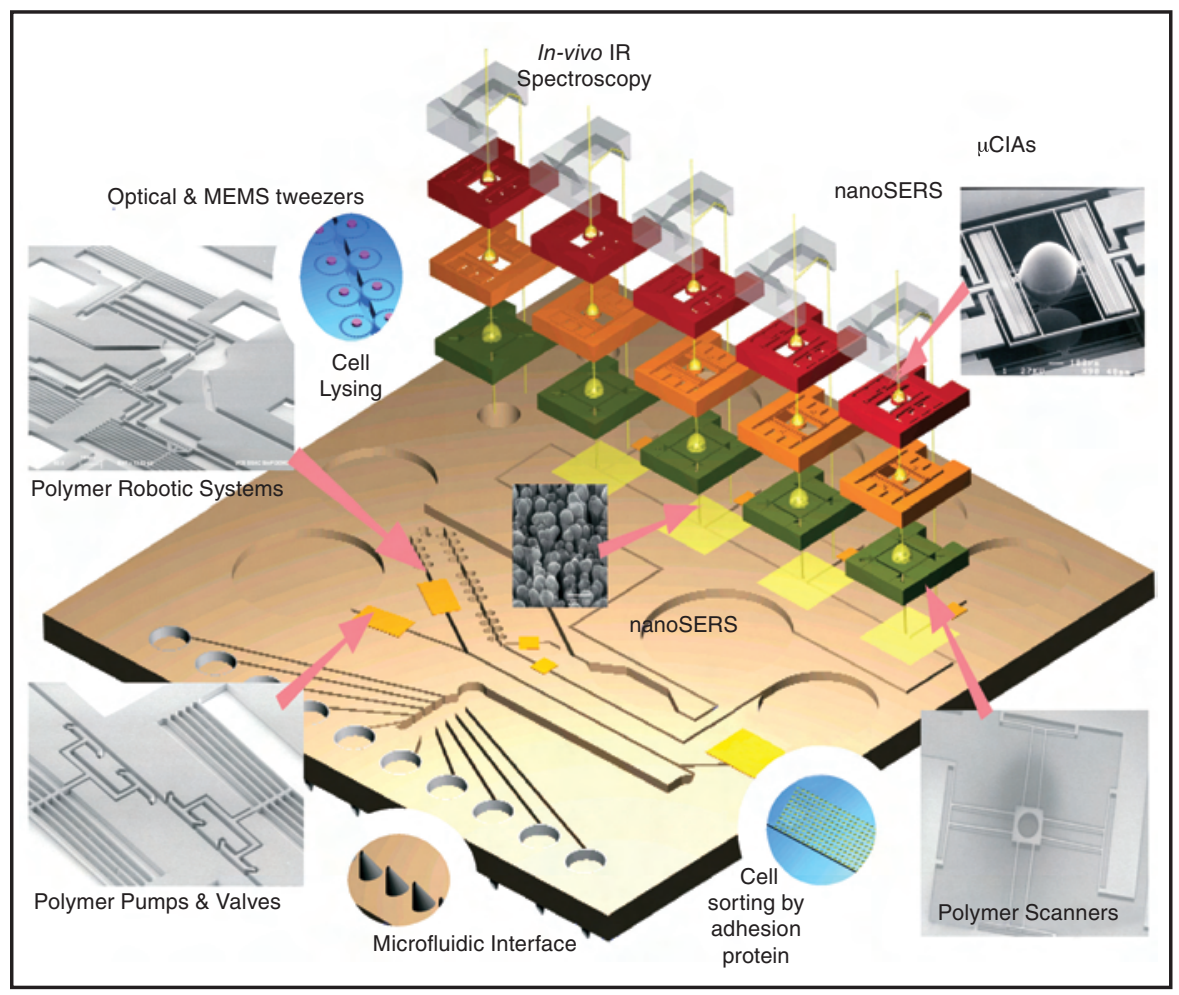

FIGURE 5.6. "Laboratory on a chip" includes an array of fluidic channels and ports for movement of chemicals. Optical and electronic detection systems, including nanoSERS (nano surface-enhanced Raman spectroscopy) and $\mu \mathrm{CIAs}$ (micro confocal imaging arrays), may be integrated as well into a truly hybrid device, combining elements from both biological and non-biological sides of the nanoscience revolution.

\section{Building Nanoscale Assemblies}

At the nanometer scale, a large number of forces are often simultaneously important. Electrostatic attraction and repulsion, chemical bonding, surface tension, and random forces from environmental fluctuations all compete to determine the structure in a mix of nanoscale objects. Depending on these interactions, nanoscale objects can be ordered, disordered, or something in between (Chapter 3). At the simplest level, these forces can be used to construct periodic arrays. Such artificial solids can have emergent bulk properties (Chapter 4) that reflect the nanoscale building blocks and the nature of the periodic arrangement.

Much more complex organizations and interesting structures are also possible, such as a "colloidosome," an assembly of nanoparticles consisting of a water core encapsulated by polystyrene beads. This shell will pass small nanoparticles but block larger ones. More complex structures can be formed by self-assembly of molecules, such as the natural phospholipids that make up living cell membranes or their synthetic counterparts, into membrane vesicles. These are permeable to water and many small molecules but are extremely effective at blocking ion transport. The coming decade will likely see rapid advances in our ability to construct complex cell-like structures made from a mix of organic and inorganic elements, and active support of such development is essential. This emerging technology will lay the foundation for ultimately producing some form of artificial cell.

\section{Ad-hoc Networking among Nanoscale Devices}

Communications among nanoscale devices presents a significant challenge even for the most rudimentary systems. Classical methods for individually connecting and configuring each element within a network (as is done in the CMOS technology in computer chips) become impractical very quickly as devices become small in dimension and large in number. It will be essential for devices to network and configure themselves. This idea of ad-hoc networking certainly exists and is even in use today. Large groups of computers and peripheral devices readily form networks among themselves. But these existing strategies do not extrapolate to nanoscale devices, which will likely function and communicate in very different ways. 
Many interesting examples of ad-hoc networking can be found in the biological world. These provide hints as to alternative ways large numbers of nanoscale devices may be able to interact among themselves. Quorum sensing among bacteria is a behavior wherein bacteria secrete, detect, and move towards higher concentrations of signaling molecules. As a result of a sensing and signal-transduction network based on chemotaxis, such as that mentioned earlier in this chapter, a wide variety of collective and seemingly intelligent group behaviors emerge. Biological signaling networks not only transduce information, they also perform logical operations on information. In this way, the program for group behavior of bacteria is contained within the functionality of their signaling network; that is, hardware and software are not separate. Quorum sensing allows bacteria to find their way through microscopic mazes just as it allows groups of bacteria the size of small countries to move and behave essentially as a single organism (Figure 5.7). It is clear that networking communications among nanosystems presents a major challenge. Correspondingly, a broad and significant effort is going to be needed to realize coordinated group functionalities, which ultimately represent some of the most powerful applications these technologies can offer.

\section{CONCLUSIONS}

This chapter has addressed the challenges of realizing the potential of nanotechnology. At the first level, we consider what is realistically possible and find that biological systems provide an existence proof that a vast array of amazing capabilities are achievable within the laws of physics. Biological systems may also provide guidance in our design of synthetic nanotechnology with similar desired capabilities. Biology, however, does not define the limits of nanotechnology. Indeed, the original applications of the word nanotechnology have generally referred to solid-state and inorganic systems. We are now at a convergence point where this classical and still rapidly growing branch of nanoscience meets the newer realization that biology is a form of nanotechnology as well. Taken together, this merged vision of nanoscience presents us with many new scientific challenges and promises even greater rewards as we gain control of these nanoscale systems.

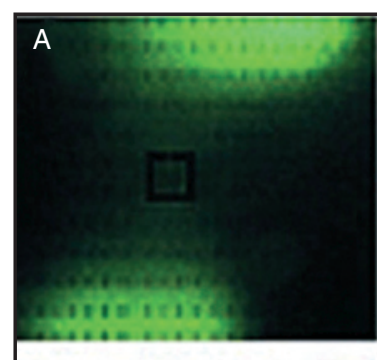

B

B thatiming iniming ए anis WW. W.

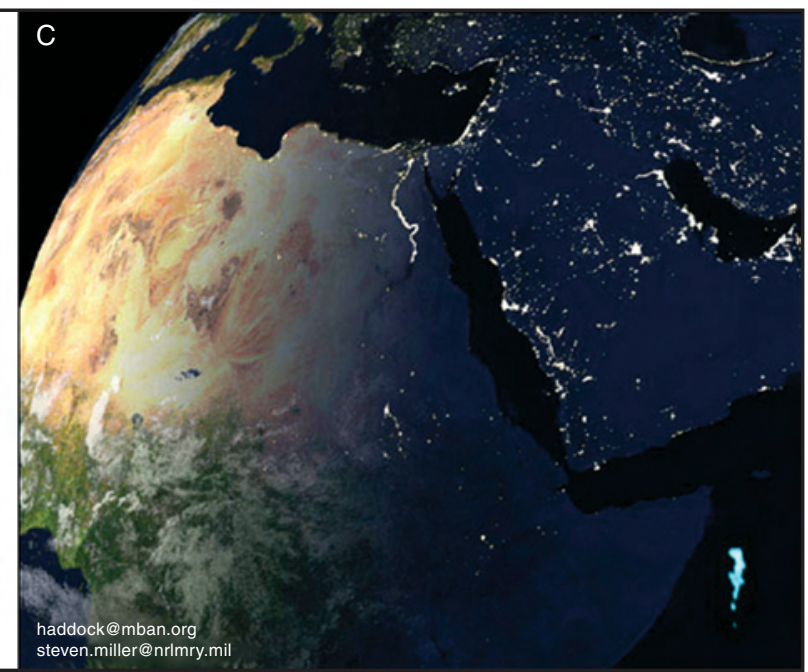

FIGURE 5.7. Bacterial quorum sensing from micrometers to megameters. Left: Fluorescence image of fluorescent bacteria (green) moving through a microscopic maze. Ultimately, the bacteria find their way to the center and become concentrated there. This is achieved by following a simple chemotaxis program encoded in their signaltransduction systems. Right: A similar phenomenon, as imaged from a satellite, is thought to occur among photoluminescent bacteria (bright blue feature, lower right) off the east coast of Africa. 


\section{MATTER FAR BEYOND EQUILIBRIUM}

The Grand Challenge for Matter Far Beyond

Equilibrium is to characterize and control matter

away-especially very far away-from equilibrium.

We live in a world of constant change. From atomic collisions to erosion of mountains, change is omnipresent. Usually, systems do evolve towards equilibrium—cold water and hot water mix to form warm water, copper roofs turn green, a child's swing eventually stops swinging. Equilibrium is the state of matter in which the forward rates of all processes equal the reverse rates for the same processes, so that there is no net change. At equilibrium, we can make many significant statements about what can happen, about the states of matter and of energy, and about the structures that occur.

Change is not always followed by equilibrium. Energy can be added to keep the swing going, or tropical storms can take thermal energy from the warm ocean. When systems are not at equilibrium, some of the most powerful science that we have, from thermodynamics to statistical mechanics, can become almost useless. This raises practical problems from how efficient a biological motor can be to how stable a glass is. But it also raises a fundamental Grand Challenge: how do we characterize and understand matter and information systems away (especially very far away) from equilibrium? These problems arise across natural and synthetic systems (see Sidebar "A Variety of NonEquilibrium Systems"), and our understanding of them is still very rudimentary.

The understanding of non-equilibrium behavior of physical, chemical, biological, atmospheric, geological, and even astronomical phenomena is a grand scientific and engineering challenge. Moreover, it is one whose solution can lead not only to huge advances in our understanding of nature, but also to technological payoffs in areas as different as earthquake and tsunami prediction, manufacture of super-hard, super-strong, and self-repairing materials, and control of crystalization and of financial panics. Understanding non-equilibrium is required for optimizing processes for obtaining, transducing, and storing energy from wind to geothermal to nuclear to fossil fuel to batteries and fuel cells. It is therefore crucial for energy supply and security. This Grand Challenge has been recognized for a long time, and solutions to it have advanced, but at the start of the 21 st century, it remains one of the chief fundamental science aims of the society.

Figure 6.1 shows part of a wind farm in the California desert. Wind turbines convert the kinetic energy of the wind to electricity. Now imagine two situations, both occurring on a day when the wind has been blowing steadily at 40 miles per hour (MPH) for a few hours, and the wind turbines are rotating nicely. In the first situation, suppose the wind blasts through at 200 $\mathrm{MPH}$ for one second. The second situation has exactly the same amount of wind energy, but this time at 42 MPH. For the energy to be the same, the 42-MPH wind will have to keep up for about 2 hours.

In the first instance of the wind-blast at $200 \mathrm{MPH}$, we would hear a lot of noise, but, owing to the mismatch between wind speed and rotor motion, not

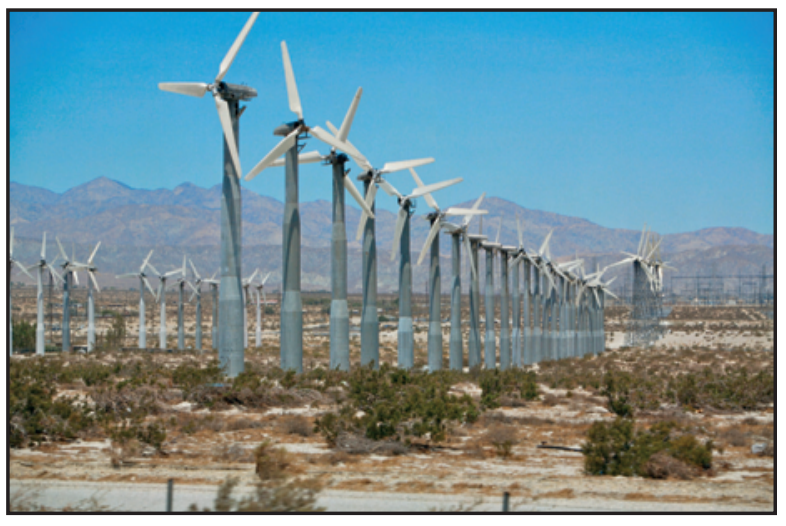

FIGURE 6.1. A wind farm in the California desert converts wind to electricity. 
much electricity would be generated. In the second instance, nearly all of the additional wind energy could be converted, depending on the design of the wind turbine.

The chief difference between these two situations is that the blast of 200-MPH wind is clearly far from equilibrium with the blades rotating in the $40 \mathrm{MPH}$ wind, while the 42-MPH wind is much closer to equilibrium. We can actually calculate the electricity produced in the second situation, using results from simple models. But for the blast, not only is the calculation far more difficult, but the net result is a pretty small jolt of electricity. This indicates two aspects of phenomena occurring away from equilibrium: we have limited ability to calculate or quantitatively predict what will happen, and the energy transduction is generally smaller than would occur at situations of equilibrium (or very close to it).

Conceptually, understanding non-equilibrium processes and systems will require addressing the major difficulties associated with bridging theories across many length and time scales-from the atomic scale and the molecular scale of water to the function of the human heart; from the femtosecond time scale associated with breaking the hydrogen bond to the days, weeks, and months characterizing atmospheric phenomena. New concepts and approaches will be needed, so that (for example) we see combustion as more than a series of coupled chemical reactions. This is not just the standard multiscale problem of computation, but rather the challenge of constructing meaningful statements and organizing principles to describe nature most completely over the many relevant scales of time and of size.

Chapter 3 stressed that conditions during most chemical reactions are far from equilibrium, so that mechanisms of reaction involve issues of dynamics and energetics away from equilibrium. The strong correlations described in Chapters 2 and 4 can affect behaviors both at and away from equilibrium, and the nanoscale processes of Chapter 5 often prepare states that are metastable, but far from equilibrium. Thus, as Figure 1.1 suggested, behavior beyond equilibrium encompasses many topics involved in all of the Grand Challenges.

\section{THE CHALLENGE OF MATTER FAR FROM EQUILIBRIUM}

\section{The Limit-Equilibrium}

Much of our understanding of nature is based on the concept of equilibrium. The idea of equilibrium underlies classical thermodynamics, which started with the steam engine, and eventually became a tremendously powerful scheme for comprehending principles of equilibrium, efficiencies of devices, energy flows in chemical and physical processes, and statements about the impossibility of certain imagined processes. As we look at the world around us, we see many examples of equilibrium systems, ranging from a perfect salt crystal, to a glass of wine, to a gold coin.

But many fascinating systems are far from equilibrium. These range from very large systems, such as weather patterns or ocean currents that remain far from equilibrium owing to an influx of energy (in this case very large amounts of heat), to biological structures from humans to horseflies whose very existence requires the maintenance of non-equilibrium conditions through the consumption of energy, to human-scale entities such as sand piles or window glass or road cuts for highways, to ultra-small structures such as nanoparticles or single cells. Non-equilibrium includes nearly all of functional biology, from processes that store energy by pushing ions against a gradient to complex networks that carry out, for example, metabolic processes operating in all creatures great and small (see Sidebar "A Variety of Non-Equilibrium Systems").

Innovations in the $19^{\text {th }}$ century led to the first important ideas in equilibrium thermodynamics and statistical mechanics from Boltzmann, Maxwell, and Gibbs. In the $20^{\text {th }}$ century, beginning with Einstein, the thermodynamics and statistical mechanics of equilibrium systems were clarified, as were fluctuations occurring at equilibrium. At equilibrium, statistical mechanics very clearly defines the relationship between the probability of a state and the entropy of that same state. While dynamics may occur at equilibrium (the carbon atoms in the perfect diamond are vibrating), the thermal distribution provides all necessary information on the state of the system. 


\section{A VARIETY OF \\ NON-EQUILIBRIUM SYSTEMS}
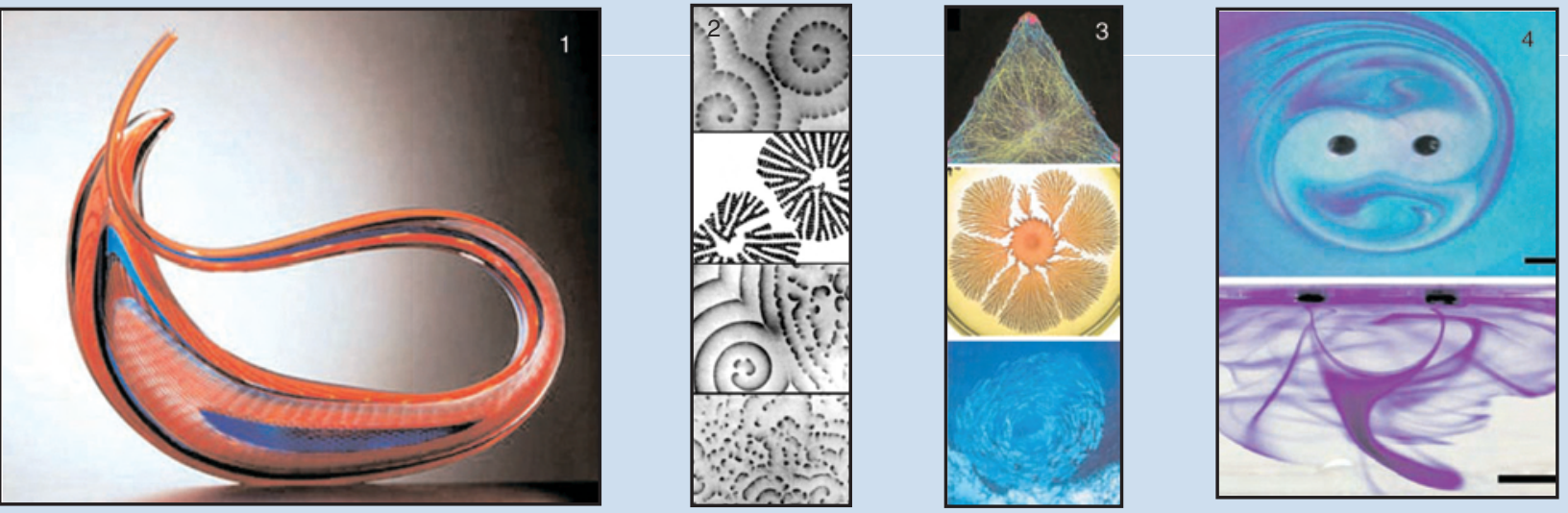

Glass sculpture (1). This is one of the many forms of art that rely on non-equilibrium. The glass is a super-cooled liquid, relaxing on many differing timescales, approaching equilibrium but never attaining it.

\section{Belousov/Zhabotinsky oscillating chemical reaction in a microemulsion (2). These} spatial patterns are observed when the initially mixed chemical reagents diffuse to form patterns (without stirring). Other similar patterns (in space and time) are observed in many systems whose dynamical equations are nonlinear.

Dynamic Self-Assembly (DySA) (3-6). Spontaneous formation of organized structures results from discrete components interacting with their environment by dissipative processes (diffusion, reaction, heat transfer, etc.). To maintain itself, the system must be displaced from equilibrium by supply and dissipation of useful energy. Far from equilibrium, these systems are capable of spontaneous organization, adaptability, self-healing, and even self-replication.

- Nature's preferred way of building its animate creations on various length scales (3). Top: fluorescently labeled microtubules in a cell confined to a 40 micron triangle on a surface of gold $\left(10^{-5} \mathrm{M}\right)$; Middle: Fractal bacterial colony $(0.1 \mathrm{M})$; Bottom: School of fish (10 M).

- Magnetohydrodynamic self-assembly (4). Two magnetic particles rotate at an airwater interface when driven by a rotating magnet. Eventually they reach a non-equilibrium steady state where the magnetic force balances the hydrodynamic repulsion. This simple system combines a conservative confinement force with dissipative in-

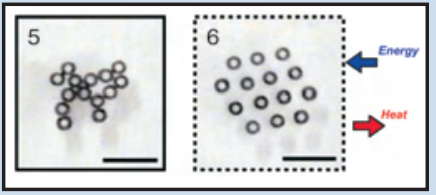
teractions. Is there an extremum principle describing this non-equilibrium steady state? Scale bars are $2 \mathrm{~mm}$.

- Spontaneous ordering $(5,6)$. At equilibrium, a collection of millimeter-sized magnetic particles showing DySA float on a liquid-air interface in a "clump." If one supplies energy by rotating a magnet below, the particles assemble spontaneously into an ordered array.

Icelandic poppy (7). Closing at night and opening in the sunlight, the poppy provides an example of circadian rhythms - a cyclic behavior driven by external inputs (in this case sunlight).

Michelle Kwan (8). This champion ice skater represents a highly non-equilibrium system that uses energy inputs to perform useful work by processes whose efficiency is difficult to characterize and whose functioning depends on non-equilibrium at many levels. Such self-organizing complexity is perhaps the ultimate challenge to our understanding of non-equilibrium systems. 
Many simplifications obtain at equilibrium. First are the state functions (volume, pressure, free energy, entropy) that allow calculations of energy quantities, the stability of structures, and the extent to which chemical reactions can proceed. In systems with $10^{23}$ particles (the typical content of a few cubic centimeters of almost anything), thermodynamic characteristics can be given in terms of a very small number of state functions, which describe not only how the given system behaves, but also how two different systems, when mixed, will evolve. The requirement of equilibrium defines extremum principles, such as the second law of thermodynamics or the variational principle of quantum mechanics, that again can be used to determine structure. A much-reduced set of macroscopic quantities, most of which are intuitive, can then characterize a system of huge spatial extent.

In quantum mechanics, the variational principle permits us to calculate the structures of molecules and of materials. These structures can sometimes be calculated more accurately than they can be measured, and from such structures we can glean very useful information on how to build new molecules and new materials, for applications ranging from medicine through metals to improved fibers for stronger, lighter, and self-repairing materials. Under non-equilibrium conditions, all of these advantages must be re-examined.

\section{The Challenge: Non-Equilibrium}

Under many conditions equilibrium does not occur. The simplest of these is when a gradient of some kind acts across the system - for example, an iron bar with one end in ice and the other end in boiling water has a heat flux across it, and equilibrium is not reached, even once a steady state heat flow is established in the bar. Far from equilibrium, even defining a temperature can be difficult.

States that are not at equilibrium, but are close to it, were analyzed in the $20^{\text {th }}$ century, beginning with Einstein and with Onsager, who established the reciprocal relationships between response properties and pointed to the close link between fluctuations at equilibrium and dissipation in the approach towards equilibrium. These principles were later formalized by Kubo and others in developing a linear-response theory that describes transport coefficients. With the growth of computational science, trajectory calculations could be used to determine correlation functions, which could also be measured experimentally. The comparisons were extremely favorable, and linear coefficients describing, for example, electrical conductivity, magnetic susceptibility, thermoelectric power, diffusion, and other processes in materials were very well established.

But when a system is far from equilibrium, this formalism fails. While we can still follow the motions of particles or other subunits of the systems, we have not yet discovered principles that allow us, without calculating the specific dynamics, to make general statements about the state of the system, or which structures are favored, or what macroscopic quantities can describe the system, or how the system absorbs or emits energy.

One of the fundamental challenges facing science in the $21^{\text {st }}$ century is understanding systems away from equilibrium. While specific instances can be cited, in which both experiments and modeling are leading to some understanding, theoretical approaches to nonequilibrium remains extremely challenging. First, we have no general principles, although major progress has been made in specific areas (such as developing expressions for work terms along non-equilibrium and equilibrium paths and for particular kinds of steadystate thermodynamic and statistical analysis). Second, some of the strongest weapons in the theoretical arsenal fail, away from equilibrium. The only techniques that still generally work are kinetics and dynamics, both quantum and classical.

Non-equilibrium situations include isolated or nearly isolated systems. Most of those discussed in this context are the obvious ones-glasses, fogs, Hollandaise sauce, proteins, polymer blends, and oscillators ranging from playground swings to oscillating chemical reactions. Non-equilibrium also occurs in systems exchanging matter, energy, or information with their environment. These include simple stirred systems (where energy is added by the stirring) weather patterns, feedback systems, directed evolution, and living systems at many length scales. The most challenging 
and significant non-equilibrium system is life itself. Others include driven systems, arrested relaxation, feedback behaviors, consciousness, and memory. Dealing with such major issues will clearly demand new forms of both conceptualization and problem formulation. We will need to develop appropriate techniques for designing and modeling specific systems, to make the appropriate measurements and to define the appropriate set of reduced variables to conceptualize, understand, apply, and master the processes of nonequilibrium (See Sidebar "Scales of Non-Equilibrium").

Approaching the problem of non-equilibrium is sometimes referred to as "bicycling through sauerkraut." General criteria for the evolution of non-equilibrium systems, for their steady states, and for their approaches towards static behavior are difficult. Perhaps the greatest challenge is either to develop an extremum principle or to prove that one does not exist. Intense efforts in this area have proceeded for the last 50 years, including attempts based on entropy production, on energy dissipation, on constrained entropy production, and on sophisticated mathematical approaches. Obtaining general statements to deal with non-equilibrium phenomena remains a major challenge to theory and to our description of matter in this huge space beyond equilibrium phenomena. Meeting this challenge, combined with the wide spectrum of situations where there is no equilibrium, their importance, and their experimental exploration, is one of the challenging enterprises of contemporary science.

\section{The Fundamental Challenges}

Nature changes over many length and time scales. Descriptions that work at one scale may be totally irrelevant at other scales. To approach the Grand Challenge- - how do we characterize and understand matter and information systems away (especially very far away) from equilibrium-we must:

- Develop the new concepts needed for description of nonlinear structures and behaviors.

- Characterize and understand non-equilibrium pathways.
- Develop a quantitative understanding of nonequilibrium dynamics, processes, and configurations in terms of a (drastically) reduced number of variables $\left(10^{23} \rightarrow\right.$ few $)$.

- Link up concepts for a broad range of length scales from the atomic to the macroscopic and time scales from the femtoseconds it takes to break a bond to the hours and years it takes a human body to mature.

- Deal with inputs of new fluxes (energy, information, matter) and predict dynamical evolution and approach towards steady states and equilibrium.

- Model amplification, selection, combinatorics, complexity, feedback and—eventually—-life itself.

\section{Points at Issue, Targets for Progress}

Understanding, describing, and controlling nonequilibrium systems constitute a fundamental challenge. But there are a number of more immediate and more specific situations that arise in contemporary science that both are part of and depend upon these major non-equilibrium issues. They may also lead to realworld advantages in systems and devices. Some of these questions are:

[1] With the advent of single-molecule dynamical experiments, we can now observe the time trace of a single molecule and compare it to the predictions of ensemble statistical mechanics. Many systems (more than we had expected) exhibit long-time tails in the way in which the motion of molecules is correlated over time, suggesting that these systems have microscopic relaxation time scales that are very long. Some of these are nanoparticles, and some are single enzyme molecules. How can we deal with these observtions, and what general rules apply (discussed in Chapter 2)?

[2] Can control of geometry at the nanoscale influence or direct structures and processes? Realizing the promise of nanoscience requires that we deal with non equilibrium and fluctuations (discussed in Chapter 5). Important processes in the geosphere can crucially depend on their non-equilibrium nature (see Sidebar "Nanominerals and Earth Chemistry"). 


\section{SCALES OF \\ NON-EQUILIBRIUM}

These structures, which extend in size from 10,000 kilometers to a million billion times smaller, demonstrate the range of phenomena in nature that occur under far-from-equilibrium conditions. All of these structures persist in this non-equilibrium geometry for a substantial time, but still they are unstable with respect to a different, true equilibrium configuration (the hurricane will dissipate, the oscillators will homogenize). Thus both equilibrium and non-equilibrium behaviors must be understood, if we are to comprehend both how nature works and how synthetic systems can be created and optimized.

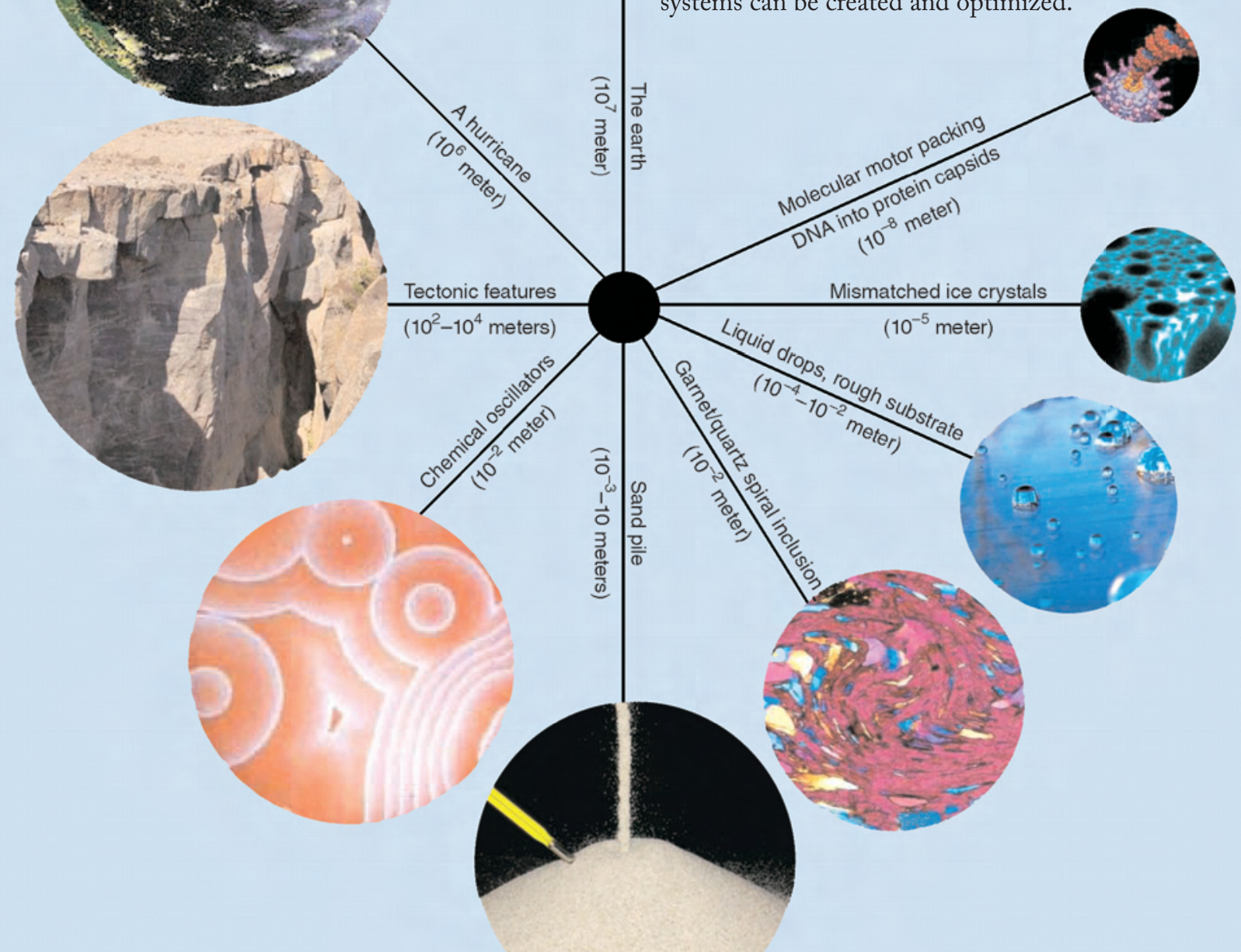


[3] Can we understand enough about biological repair mechanisms to use them (or related schemes) in molecular and solid-state device applications - that is, can we make a self-repairing computer? Or a selfrepairing molecular transistor? Or self-repairing lighting? Solar cells are subjected to light with an effective temperature of $6000 \mathrm{~K}$, so that self-repair will be crucial - can we understand how to achieve it (discussed in Chapters 3 and 5)?

[4] Can we comprehend how systems search freeenergy landscapes? This territory includes structures from molecular recognition to glasses, and kinetics from assembly to stability (discussed in Chapters 2, 3, and 4).

[5] Can we develop efficiency measures for cellular processes, for artificial cellular processes, and for highly non-equilibrium processes to extend the standard Carnot efficiencies of equilibrium systems? How do we design machines to operate far from equilibrium to produce power and to do so efficiently? Is there an effective non-empirical approach for these problems?

[6] Can we understand non-equilibrium processes in biology, such as kinesin/actin motions. entropy reduction. quorum sensing. or active transport within cells, well enough to mimic them with artificial systems (discussed in chapter 5)?

[7] Can we use the multiple-temperature phenomena, associated with very slow relaxations of particular subsets of modes, for energy control? In particular, can we utilize artificial structures with such modes to store excitations long enough for energy transduction from vibrational to electronic and thereby increase substantially the efficiency of solar capture devices (discussed in Chapters 2, 3, and 4)?

[8] Can we develop general methods for stabilizing matter in particular non-equilibrium states such as metamaterials, or high-strength glasses, or graded structures (discussed in Chapter 3)? People have made glass for millenia, but can we deal with the general problem of making stable non-equilibrium structures?

[9] Can we understand and manipulate turbulent flows coupled with phase transitions, diffusion, and chemical reactions? This capability is required for problems as different as climate prediction, tidal flow, or combustion (discussed in Chapter 4)?
These issues and possibilities arise from the current state of understanding of the phenomena, processes, and materials of non-equilibrium systems. The remainder of this chapter is devoted to delineating both the state of that understanding and some of the many aspects of non-equilibrium systems - of great importance to Basic Energy Sciences, to our developing processes and materials to secure our energy future, and to our comprehension of nature- that remain unclear and challenging

\section{ACCOMPLISHMENTS, APPROACHES,} AND AREAS

The fundamental challenges just listed appear in various places in the real world. Areas of specific interest and application presented here are particular manifestations of the non-equilibrium challenge.

The largest-scale issues arise in understanding the structure and dynamics of the atmosphere. The old saying that nobody understands the weather reflects the deeply challenging problem of climate prediction arising from the tremendous complexity of the systems involved. Hurdles include the interaction of atmospheres, earth surfaces. and oceans; diffusive patterns and chemical reactions; surface chemical phenomena in clouds and on particles; and heat and energy flows from sunlight; and turbulent mixing. Weather systems (like the cyclone in Figure 6.2) are never in equilibrium either globally or locally - when the atmosphere is heated in one place and cooled in another (far away), it generally does not go to steady state.

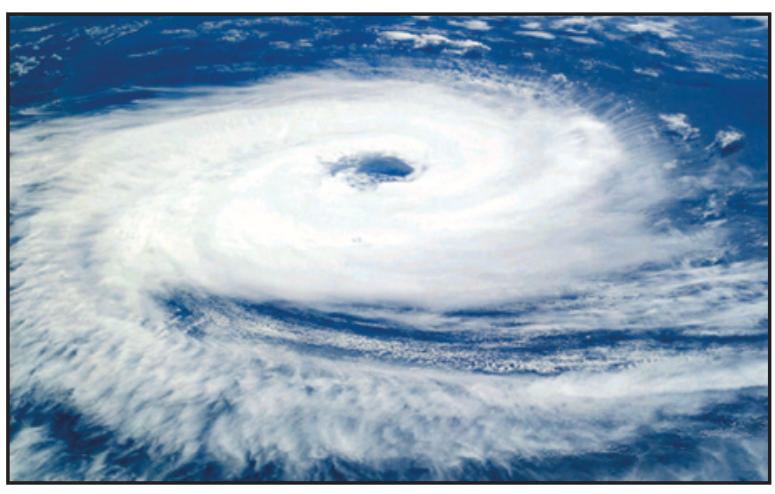

FIGURE 6.2. Cyclone Catarina, a South Atlantic tropical cyclone, viewed from the International Space Station on March 26, 2004. 


\section{NANOMINERALS AND EARTH CHEMISTRY}

The Earth is a magnificent tangled web of systems that are not in equilibrium within themselves or with one another. Although this disorder may at first seem disconcerting, it is precisely what keeps this planet dynamic and alive. These non-equilibrium processes involve more than 4,000 mineral species. Minerals are created, destroyed, and recycled on Earth, and they form the substrate upon which life is sustained. They provide most of our natural resources; they give up the phosphorous (and many other essential elements) necessary for life; and they are the raw material from which soil originates. None of these processes could occur if minerals were at equilibrium with the geosphere.

Like other materials from metallic silver and gold to semiconductors, these minerals, when present as nanoscale particles, show sizedependent properties. When individual grains of minerals approach the size of molecular clusters of atoms that are measured in nanometers $(\mathrm{nm})$, they behave differently, often dramatically differently, than the same mineral in larger sizes. We call these nanominerals, and we find them everywhere, from deep oceans to the upper atmosphere.

- The smallest of the nanominerals can be seen with a scanning tunneling microscope and are only a few atoms across (1). The blue-white mounds are individual iron atoms on the surface of a pyrite crystal. The black patches, only 1 to $2 \mathrm{~nm}$ across, are ironsulfate nanominerals. The formation of these minerals generates acid, which is the origin of acidic mine drainage, a very serious environmental problem.

- Nanominerals can be effective catalysts, an example being 5 to $15-$ $\mathrm{nm}$ grains of titanium oxides found in the Clark Fork River in Montana (2). This river flows through the largest Superfund complex in the United States, whose pollution results from a century and a half of mining activity upstream.

- Bacteria interact with nanominerals (3). Shewanella odeinensis is an important bioremediation species. It respires using metal ions from the environment, whose oxidation states are reduced in the process. This is a promising way to deal with such pollutants as chromium or uranium.

- We even drink nanominerals (4). This cluster of iron oxide nanominerals 5 to $10 \mathrm{~nm}$ in diameter, here carrying lead, was recovered from the water supply of a major U.S. city.

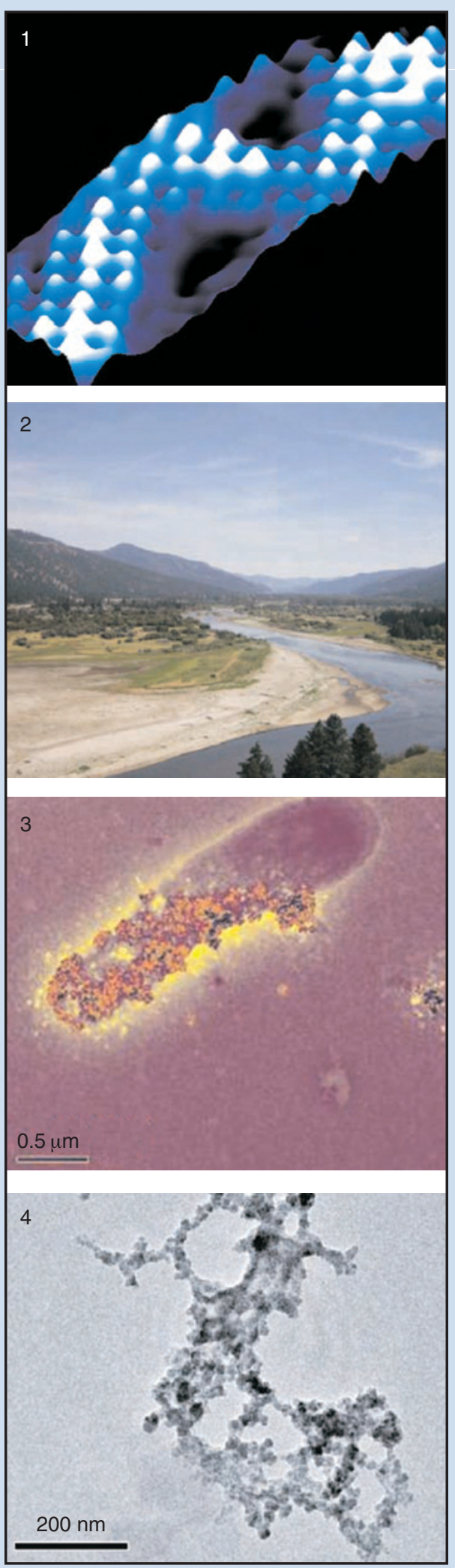


In ocean currents and marine systems, similar situations arise, except that the density of the medium is a thousand times larger. Non-equilibrium again emerges from the tangling of energy flows, complex dynamics, and thermal and density (salination) gradients. Turbulence and phase transitions are extra complications. Turbulence is also relevant in understanding stability of flow systems. This has both obvious effects on daily life (wind patterns, floods, pump failure, noise, tornados) and fearsome mathematical challenges.

At the smaller scale of the laboratory or the objects of everyday life, non-equilibrium situations provide a rich phenomenology, and new issues arise. Arrested relaxation is found in systems such as glasses, glassy polymers, or glassy metals. These systems appear to be at steady state, but in fact are relaxing on a very broad distribution of time scales toward states that are poorly defined both experimentally and computationally. In such metastable systems, relaxation processes, structural evolution, and constitutive processes remain a tremendous challenge. In the important science (and technology) of electrochemistry, very few reactions truly occur at equilibrium, but such concepts as overpotentials and quasi-reversible reduction-oxidation processes provide a useful and practical set of techniques for quantifying and understanding these processes, even away from equilibrium. When dealing with structural and chemical transformations in condensed matter, engineers and condensed-matter scientists have developed methods for growing materials under strongly non-equilibrium conditions - this is how some of the strongest and most useful materials, from plastics to superalloys, have been prepared and characterized.

Oscillatory chemical processes extend simple chemical reactions to include circadian rhythms in biology and other self-organized temporal structures in systems of widely varying size. Conditions of energetic excitation are a classic instance of dynamics far from equilibrium. These can include something as simple as a stirred suspension of particles in a fluid, but dynamical self-assembly is perhaps the most striking example (see Sidebar "A Variety of Non-Equilibrium systems"). Biology is dominated by processes far from equilibrium, many of which are controlled by inputs of new fluxes (of materials and information and energy — see Chapter 5). The richness and diversity of life arises from the complexity of non-equilibrium systems under these conditions.

At the nanoscale, characteristic non-equilibrium behaviors can be observed directly, without the averaging over many molecules or subsystems that are seen in laboratory-scale measurements. These behaviors include single-molecule spectroscopy and fluctuations of small systems, finite-size effects and dissipative structures at the nanoscale, and dynamical self-assembly and dynamical dissipation (see Sidebar "Thermodynamics of Molecular Machines").

\section{A Simple Example: the Molecular Transport Junction.}

Steady-state systems in gradient fields are classic examples of stability obtaining far from equilibrium. A simple and beautiful (and much bigger!) example is Niagara Falls. A second example, the subject of extensive current research, is transport in molecular junctions, from the nanostructured single molecule to conductive polymers.

Imagine a single molecule suspended between two continuum (semi-conductor or metal) electrodes. Suppose, for simplicity, that the molecule is bound to the two electrodes by only one atom at each end. Now imagine that the electrodes are placed under potential bias, and current begins to flow from the cathode to the anode through the molecule. One can then measure a current/voltage characteristic for the structure, and one might ask how to explain both the structure and the process. This situation is shown in the Sidebar "Molecular Transport" of Chapter 5; it involves both the challenge of non-equilibrium and that of nanoscale communication (and parts of strongly correlated systems).

Landauer's work in the 1950s considered a closely related system in which there is an unspecified channel between cathode and anode. Landauer showed that the current (very unexpectedly) is quantized. Even in a perfectly conducting channel, the conductance is finite, given in terms of fundamental constants, including Planck's constant and the electronic charge. It is not behaving in accordance with the Ohmic considerations that describe most near-equilibrium macroscopic structures. 


\section{THERMODYNAMICS OF MOLECULAR MACHINES}

Thermodynamics describes how energy is exchanged in systems; gases, liquids, magnets and superconductors comply with its laws. The observed behaviors of macroscopic systems such as steam engines are reproducible and fluctuations (deviations from the typically observed or average behavior) are small. However, virtually all approaches now being considered in the energy sciences involve small systems that are not fully at equilibrium or described by conventional bulk thermodynamics.

Examples of small systems include biological molecular machines ranging in size from 2 to $100 \mathrm{~nm}$. Biological molecular machines are responsible for almost all cellular processes relevant to bio-energy. For example, light is harvested by photosynthetic machinery; the universal energy currency of all life, adenosine triphosphate (ATP), is produced by an enzyme (the $\mathrm{F}_{0} \mathrm{~F}_{1}$-ATP synthase). These molecular machines are about one billion times smaller than steam engines (Figure 1). Fluctuations are essential for their activity, and their characteristic energy scales are close to thermal energy $\left(\mathrm{k}_{\mathrm{B}} \mathrm{T}\right)$.

Scientists are now beginning to investigate the thermodynamics of molecular machines. How is it that these tiny machines operate at energies only marginally above the thermal energy of the surroundings? How do the large fluctuations experienced by these systems affect their operation? Can we learn to create artificial nano-devices with similar properties?

The development of a thermodynamic formalism applicable to small non-equilibrium systems is one of the fundamental tasks of contemporary thermodynamics and statistical mechanics. So-called "fluctuation theorems" are likely to be the main ingredients of a more general theory (see Sidebar "Fluctuation Theorems").

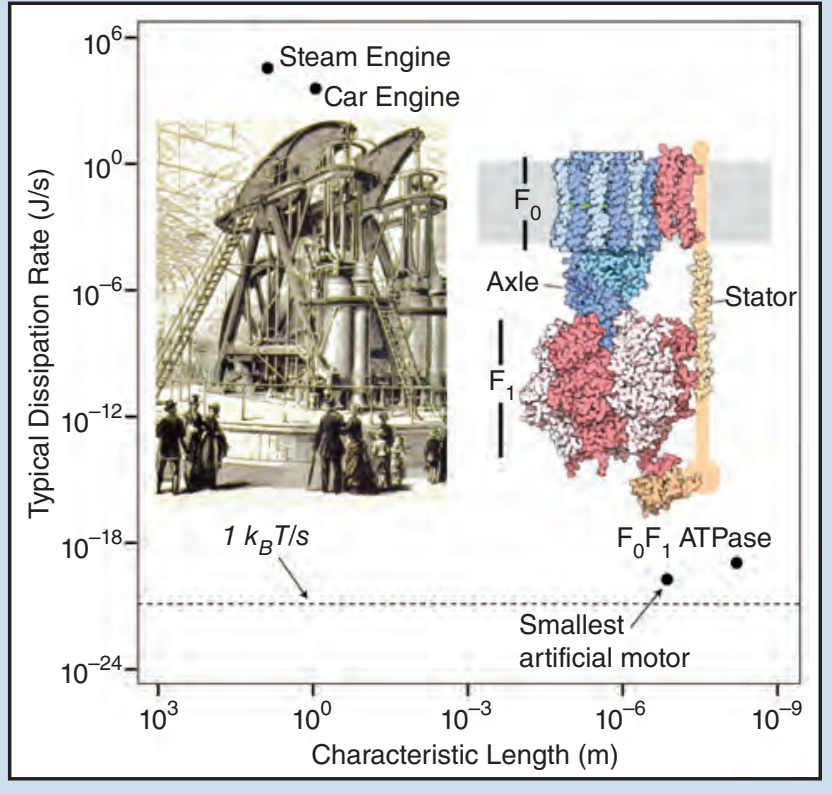

FIGURE 1. Different thermodynamic systems according to their typical length scales and dissipation rates. Conventional bulk thermodynamics was developed to treat systems in the top left quadrant of the plot; contemporary energy research focuses on systems in the bottom right quadrant of the plot. Small systems such as molecular machines are characterized by dissipation rates on order of a few tens of $k_{B} T / s$. 
It is difficult to characterize the non-equilibrium structure. The molecule is in a schizophrenic position, trying to decide whether to equilibrate with the chemical potential on its right or left, when those two chemical potentials are different. There are gradients of electrostatic potential and chemical potential across the molecule, and a current will flow. There is no principle to calculate the geometry of the junction. Indeed, the geometry of the junction can change, depending on the current that flows and in response to the applied potential. At present, there is no direct experimental structural probe to measure the geometry in the operating junction.

We could also imagine (and there is growing experimental evidence for) the system going into multiple steady states or moving among steady states in a periodic or a chaotic fashion. It is possible to write a combination of quantum and classical kinetic equations to describe the motions, but these kinetic equations, while they can yield a description of the conductance, do not characterize the evolution of the system toward a given stationary state, nor do they permit calculation of the geometry, nor do they allow calculating the conductance in a systematically improvable fashion.

Within the last decade, major progress has been made in making, measuring, and modeling such molecular transport junctions. Molecular charge transport is of great interest for applications from cheaper photovoltaics to atmospheric and process monitoring, diagnostics and therapeutics to revolutionary advances in computer efficiency and capability. Nevertheless, even in the simplest such system difficulties arise because of the non-equilibrium nature, issues that demonstrate the wide range of possible behaviors away from equilibrium.

\section{The Science of Life}

Important science has been done on static aspects of biology, such as the structures of the major biological macromolecules, including DNA, proteins, carbohydrates, and other intermediate-size structures (see Chapters 2,3, and 5). The dynamics of biological systems (that nearly always occur far from equilibrium) are far more challenging than these static aspects. Processes of dynamical self-assembly pervade biology, involving everything from DNA forming into the double helix to multiprotein complexes assembling to enable transcription and translation, RNA splicing and signaling, endocytosis, and other cellular processes. On a larger length scale, cellular membranes can selfassemble from phospholipids, cholesterol, glyco lipids and protein building blocks. These come together to delineate the cellular boundary. Cells may spontaneously organize into tissues, tissues into higher organisms, organisms into populations, populations into communities, and communities into ecosystems. The end result is the biosphere.

Biological structures develop flows, self organize, self repair, establish networks, and utilize both gradients and fluxes to keep themselves away from equilibrium-when a living organism reaches equilibrium, that organism is dead! The remarkable knowledge that we have obtained in the biological sciences constitutes deep and powerful phenomenology. Nevertheless, because the relevant behaviors occur far from equilibrium, fundamental physical principles (such as thermodynamics or variation principles— see Sidebar "Thermodynamics of Molecular Machines") are not directly useful for understanding most of the significant problems of biology from evolution to growth. Their description, requiring differing concepts at different length and time scales, and generally far from equilibrium, is a challenge of deep complexity.

\section{Fluctuations}

All molecular and larger systems constantly fluctuate. Whether in a diamond crystal or a gas-filled balloon, these fluctuations are present. When the system is pushed away from equilibrium (and if no further perturbation is applied), the response of the system can be determined by its equilibrium fluctuations, and the ratio of fluctuations to response will be proportional to temperature.

The characterization and measurement of fluctuations is a very active area of non-equilibrium science, and one of the most theoretically useful ways to characterize non-equilibrium behavior (see Sidebar "Fluctuation Theorems"). The Jarzynski relationship and Crooks' theorem define work in non-equilibrium systems (but still require the existence of a bath at thermodynamic temperature). The Jarzynski equality is 


\section{FLUCTUATION THEOREMS}

Fluctuation Theorems (FTs) describe the probabilities for a system to exchange energy with a thermal bath. Experimental techniques, such as optical tweezers and atomic force microscopy, have recently allowed scientists to test directly the validity of several fluctuation theorems.

In 2002, an FT applicable to systems arbitrarily far from equilibrium was verified by repeatedly dragging microscopic beads through water with an optical trap and computing the entropy production for each bead trajectory (see Figure 1). Strikingly, entropy-consuming trajectories were occasionally observed. This behavior was actually discovered by simulation, which predicted the local entropy reduction. Unlike bulk systems, small systems can temporarily borrow energy from the thermal bath and use it to reduce their entropy. Of course, on average, the entropy of the system increases according to the Second Law of Thermodynamics.

There are several fluctuation theorems, each applicable to slightly different thermodynamic systems. The Crooks fluctuation theorem and the closely related Jarzynski equality are directly applicable to mechanically perturbed small systems such as RNA hairpins or single proteins. The Jarzynski equality asserts something remarkable. If you take a small system and drive it many times from state $\mathrm{A}$ to state $\mathrm{B}$, and if you perform a particular kind of averaging over those trajectories, you can recover the free energy difference between A and B, regardless of how violently the system is perturbed (Figure 2)! Like other fluctuation theorems and closely related results, the Jarzynski equality relates equilibrium thermodynamics with non-equilibrium thermodynamics - it is a bridge between those two fields.

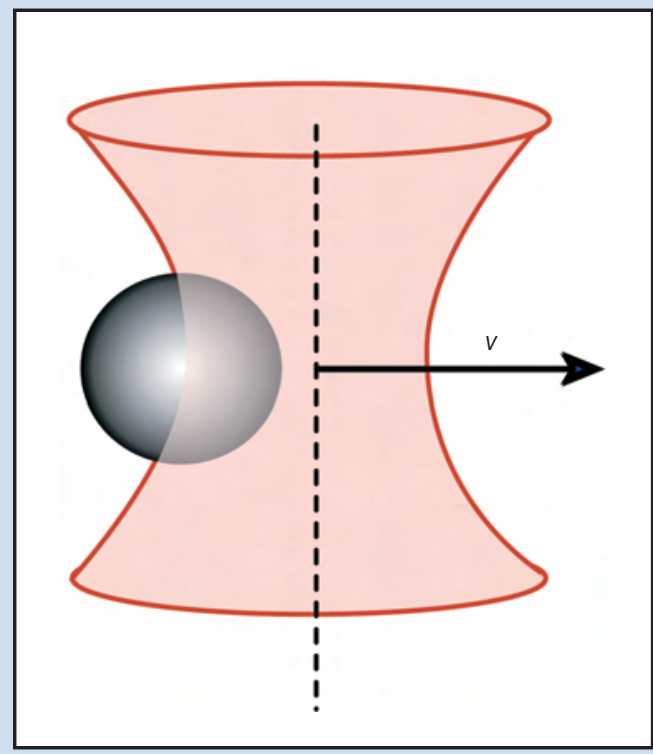

FIGURE 1. Microscopic beads in an optical trap. The pink represents the trap, the black ball is the bead; and $v$ is the velocity of the bead.

$$
\begin{aligned}
& \text { The Jarzynski Equality } \\
& \left\langle e^{-\beta W_{d}}\right\rangle=1
\end{aligned}
$$

where $\beta=1 / k_{B} T$ and $W_{d}$ is the dissipated work

FIGURE 2. The Jarzynski Equality.
The Jarzynski equality has been tested by mechanically stretching a single molecule of RNA, a non-harmonic dissipative system, reversibly and irreversibly between its folded and unfolded conformations. Depending on how rapidly the hairpin was unfolded, different amounts of work were needed. When the polymer was unfolded slowly, the average forward and reverse trajectories could be superimposed, indicating a reversible reaction. When the polymer was unfolded more rapidly, the mean unfolding force increased. The folding-unfolding cycle was thus hysteretic, indicating the dissipation of work. And yet, application of the Jarzynski equality to the irreversible work trajectories yielded an excellent estimate of the free energy of unfolding process.

Fluctuation theorems are not only tools that experimentalists can use to extract equilibrium information from non-equilibrium experiments, but are also useful to comprehend the properties of non-equilibrium systems and may ultimately guide the design of such systems. Now that it is possible to test fluctuation theorems and readily evaluate their performance, the next decade will likely see significant advances in our ability to model, understand, and harness small non-equilibrium systems. 
a substantive advance because it is a generic statement about the behavior of non-equilibrium systems that does not depend on details of the kinetics or dynamics. So-called fluctuation theorems posit that entropy is a fluctuating quantity and that the probability for a positive entropy fluctuation is exponentially larger than for a negative entropy fluctuation of the same magnitude.

Extensive simulations have been attempted to characterize fluctuations of physical systems far from equilibrium. But we still have no general methods for computing response properties of such non-equilibrium situations, in contrast to the equilibrium fluctuation/dissipation theorem. Noise spectra for systems subject to energy fluxes (such as molecular junctions) are being measured, and full counting statistics of such systems challenge analysis. Single molecule spectroscopy observes fluctuations at characteristic frequencies, changes that are due to exchange of energy with the molecular environment. Understanding the nature of such fluctuation provides insight into the dynamics and the overall causal evolution of these systems.

A very simple model of chemical-reaction kinetics assumes that the rate is simply the number of instances per unit time that the molecular entity fluctuates over the top of a potential-energy barrier separating reactant and product geometries. Many natural processes occur by such accepted fluctuations. Some sophisticated models for Darwinian evolution are based on permitting traits to fluctuate randomly, and the genotypes develop by accepted change (the survival of the fittest) from fluctuations (random mutations) evaluated by competitive success. Similar evolutionary or agentbased algorithms are beginning to be applied to such non-equilibrium problems as dynamic self-assembly and polymer dynamics. They do not solve equations of motion (unlike traditional molecular dynamics), and may be better adapted to deal with issues of noise, complexity, and multiscale environments.

The nature of the fluctuations is one index of the existence of non-equilibrium. Understanding how these fluctuations occur, measuring the evolution and fluctuations of these systems, and characterizing the approach to a steady-state or equilibrium situation is a significant aspect of the grand challenge of matter far from equilibrium. As Valentine puts it in Tom Stoppard's Arcadia:
"People were talking about the end of physics. Relativity and quantum looked as if they were going to clean out the whole problem between them. A theory of everything. But they only explained the very big and the very small.... The ordinary-sized stuff which is our lives, the things people write poetry about- clouds, daffodils, waterfalls, and what happens in a cup of coffee when the cream goes in-these things are full of mystery, as mysterious to us as the heavens were to the Greeks. We're better at predicting events at the edge of the galaxy or inside the nucleus of an atom than whether it will rain on auntie's garden party three Sundays from now. Because the problem turns out to be different. We can't even predict the next drip from a dripping tap when it gets irregular. Each drip sets up the conditions for the next, the smallest variation blows prediction apart, and the weather is unpredictable the same way, will always be unpredictable."

The problems he mentions- dripping faucets, daffodils, waterfalls, clouds, cream in coffee and weather prediction - all deal with situations away from equilibrium But part of the challenge is to make his last statement incorrect.

\section{Design Approaches-Complexity, Communications, and Robustness}

The strong analogies among physical non-equilibrium situations, biological systems, and organized assemblies (such areas as the structure of the Internet and the efficiency of markets) suggest that dynamical evolution and design in such systems can be based not only on fundamental physics, but also on different criteria for evolution and selection. Darwinian evolution, briefly mentioned in the last section, is one such principle. Others include optimization of some communications protocols among individuals (for example, in quorum sensing) and evolution dynamics based upon robustness of the assembly to external and internal perturbation.

One simple way to think of this is the analogy of a computer algorithm to the evolution of a real system. Standard computational physics approaches to temporal evolution include molecular dynamics (essentially solving Newton's equations of motion for the atoms), and Langevin dynamics (doing the same, but including 
interaction with an external thermal bath), and MonteCarlo or master-equation dynamics (assuming thermalized motion on particular potential-energy surfaces). Other algorithms, developed more recently and applied to different situations, include agent-based calculations and evolutionary algorithms. These do not start from fundamental dynamical principles of the physical systems, but rather from evolution criteria based on an arbitrary set of rules. These algorithms have been very useful for description of simple physical systems, such as chiral clustering, nucleation, nanostructure formation, and adsorption behaviors. For such problems as supply-chain management and population evolution, which are clearly difficult to represent in terms of simple physical variables, non-equilibrium situations can be dealt with very well using such rulebased simulation methodologies.

These behaviors illustrate how evolution schemes not based on the simple laws of physics can be useful. They also show how, as a system becomes more complex, these more intuitive and (in a sense) arbitrary evolution schemes become advantageous.

So-called complex systems, in which size-dependent behaviors are seen and emergent properties become crucial, are extensively discussed in Chapter 4. They are closely related to the non-equilibrium problems of interest here, since such structures almost always begin in a non-equilibrium situation. Similarly, there is extensive interest in using robustness as a criterion for determining system behavior. Essentially the basis for these arguments is that the systems that are not robust to external perturbation are like metastable situations in physics—small perturbations can push them away from their current stable state, which is therefore a poor descriptor of the system. More generally, the robustness idea uses the capability of the systems to withstand classes of external perturbation as a criterion for optimization. Common examples of such criteria involve statements like "trees that bend do not break," so that robustness against brittle fracture leads to long-term stability. Criteria for robustness are being developed and used to describe the evolution of systems far from the equilibrium state. These must be focused and made more general, if this approach is to be of real utility in understanding arrested non-equilibrium behavior.

\section{Exploring Rough Landscapes-Finding Home in the Mountains on a Dark Night}

A nearly universal model for describing the structure and dynamics of atomic, molecular, and biological systems in condensed phases involves the concept of the potential-energy surface. This is an intuitive structure in which the independent variables are the different coordinates of the particles comprising the system (for example, the hydrogen and oxygen atoms in ice) and the dependent variable is the total potential energy. The surface contains minima- that is, places where the energy can only increase upon leaving the particular point in any direction. These minimum points are stable geometrical structures for the system, but according to equilibrium theory, the relative probabilities for occupying such states decreases exponentially as their energy increases.

The similarity to a mountain range is often discussed, where the deep valleys correspond to the stable minima, and the rate to go from one valley to another depends on the difficulty of passing over a pass in the mountains. If the pass is too high, the required energy to go over it will be very large, much larger than can be obtained from thermal energies. Under those conditions, certain passes may be impassible, and going over them is therefore impossible. Once the system is trapped in a local minimum, it may take an extremely long time to go over the pass to find a deeper valley, and the nonequilibrium state is stable over that long period of time. Figure 6.3 sketches possible landscapes in one and in two dimensions. In the two-dimensional landscape, local minima are labeled by stars on one side of the dividing ridge, and by diamonds on the other. It is clearly easier to get from one star to another than to get from a star to a diamond (because the energy barrier is lower). In the one-dimensional plot, it is clear that higher barriers separate clusters of local energy minima.

We can also imagine situations where there is a very high ridge separating several minima. For example, the minima on one side of the continental divide ridge line in the Rocky Mountains are connected by passes that are lower than the ones crossing the divide. One could then have equilibrium among minima on the east of the divide, but not among all of the minima (on both sides). 


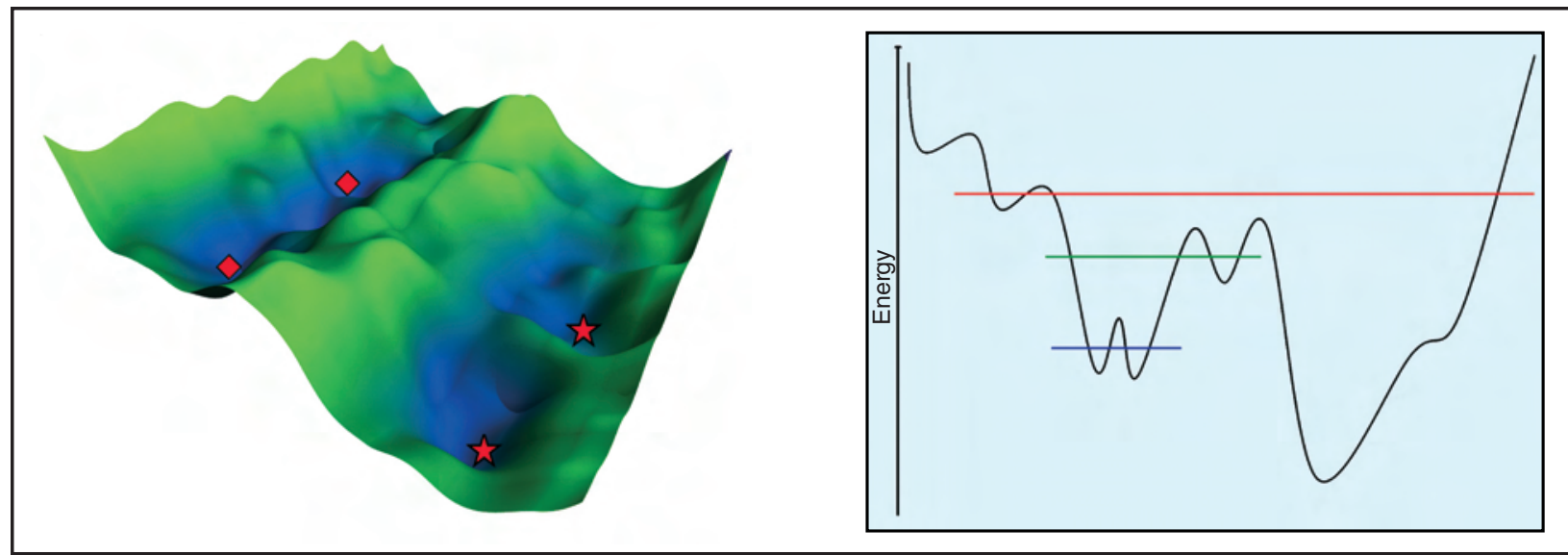

FIGURE 6.3. Schematic potential-energy landscapes, in two (left) and one (right) dimensions. Each point on the horizontal axis represents a different arrangement of the particles.

Under these conditions, once again, the system cannot find its true equilibrium state, and we have a long-lived non-equilibrium situation. This situation characterizes systems such as glasses that exhibit arrested-relaxation processes, when the thermal energies are much lower than the barriers. Proteins can also behave in this fashion, with only a subset of the low-energy valleys having the correct fold for biological function.

Physical examples of such situations abound. Common ones include long-lived molecules with one geometry that is less stable than the other geometry. For example, the two isomers of the azobenzene molecule shown in Figure 6.4 have such long term stability-the extended structure is the more stable, but at room temperature it takes months for them to interconvert. Such interconversion can be made very rapid by photo excitation - we can then have a distribution between the two isomers controlled both by temperature and by the light shining on them. This is a controlled non-equilibrium situation in which the conditions in an experiment can select the distribution desired.
Extended molecular entities in condensed phases can also exhibit long-term non-equilibrium metastabilities. Again many examples are found in the kitchen, where mayonnaise is in a non-equilibrium state that can be stable for extremely long times in the absence of external perturbations. Synthetically, one can utilize these non-equilibrium conditions to prepare (for example) super-cooled liquids such as Pyrex glass, super-strong glassy metal alloys, or stable but non- equilibrium polymer configurations such as the rod-like form of Kevlar.

In biology, improperly self-assembled (misfolded) proteins can exhibit such non-equilibrium states that exhibit long-term stability. These misfolded proteins can result in very serious disease conditions, such as Tay-Sachs, Creutzfeld-Jakob, or sickle cell disease. Misfolded proteins also characterize mad cow disease, and Alzheimer's. They, therefore, are of major importance in medicine.

Thus control of non-equilibrium can be very useful for materials and is crucial for biology. Engineering

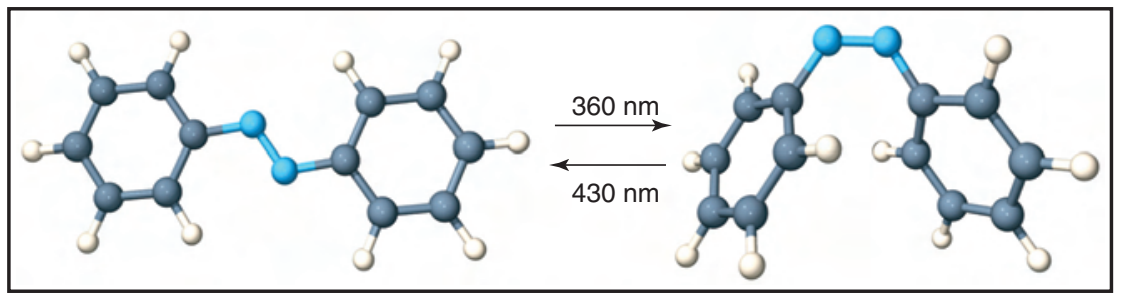

FIGURE 6.4. Photo-interconversion of two isomers of the azobenzene molecule. The direction of the interconversion depends on the wavelength of the light. 
such long-lived non-equilibrium systems requires deeper understanding of how to explore the multi-dimensional potential-energy surface in order to escape from (or perhaps to maintain) the original non-equilibrium regime. This indeed corresponds to the system's trying to find its way home on a dark night in the mountains, and characterizing this process provides a substantive challenge to both theory and experiment.

\section{No Room at the Inn-Jamming Processes}

The potential-energy landscapes comprise a nearly universal framework for discussing the geometric arrangements of atomic, molecular, and biological systems. There is an intriguing limiting situation that has unique consequences: if the only potential terms are hard-core excluded-volume interactions, like those between billiard balls, the potential-energy value (PE) for any geometry is either zero (allowed structure) or infinitely large (forbidden structure). The Boltzmann factor $[\exp (-\mathrm{PE} / \mathrm{kT})]$ is then either unity (allowed) or zero (forbidden) for any geometry, and is thus independent of temperature.

The fact that a given allowed structure may be surrounded in space by forbidden ones leads to the phenomenon of jamming. For example (Figure 6.5), if the marbles in the funnel approach the hole at different

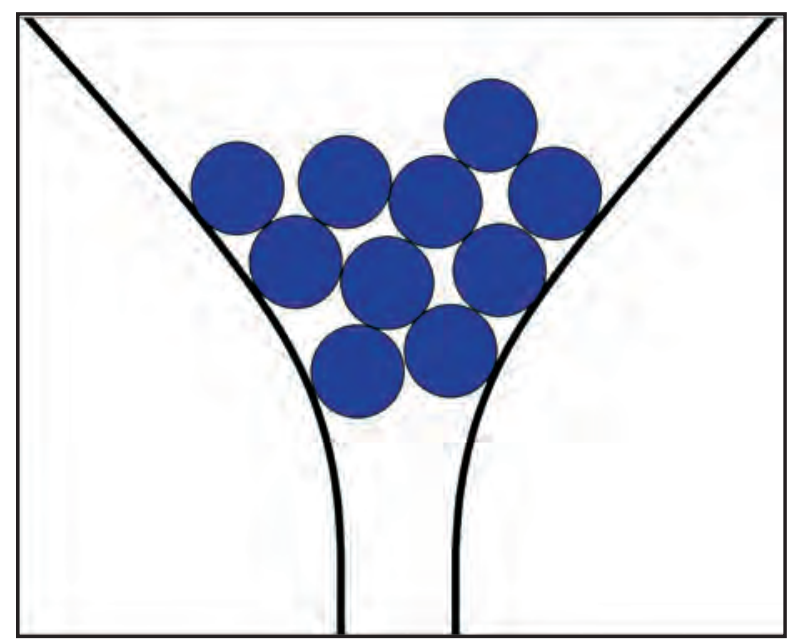

FIGURE 6.5. Marbles trapped in a funnel-one example of jamming. times, they pass through. But if they approach at nearly the same time, they get permanently stuck (jammed), although there is clearly an available lower gravitationalenergy state if they can pass through. Jamming occurs when systems cannot explore all of phase space because kinetics traps them in a jammed (fully arrested) situation.

The notion of jamming is useful for understanding the phase and geometric behaviors of quite different systems. Sand piles are classic cases, where the jamming state can be metastable, depending on the pile steepness. The motion of a single grain can cause collapse (or not). Many glasses can be thought of as jammed (solid-like physical properties, disordered liquid-like structures). Dense colloids, foams, and mixing in solids can all show jamming behavior. Jammed structures can be metastable or very stable (as sand piles show and as traffic jams maddeningly demonstrate). The exploration of geometry and of phase space for jamming systems is related to the potential-energy landscapes, but here thermal effects are far less important than shaking or stirring in modifying the structural stability.

\section{FINAL COMMENTS}

Outside of science and engineering, there are processes that also evolve in a non-equilibrium fashion. In economics, most theoretical and modeling work has been on equilibrium processes. It is recognized that actual functioning economies have important non-equilibrium components that provide their dynamism, their complexity, and their adaptability. But the theory of non-equilibrium systems in economics is still limited, largely because it is so difficult. Perhaps looking at this sister area of economics will help scientists interested in non-equilibrium structures and properties of matter at length scales from the atomic to the interstellar realize the complexity, challenge, and importance of nonequilibrium phenomena.

The widely varying instances of behavior far from equilibrium suggest that addressing this Grand Challenge will require new theoretical ideas, creative experimental approaches, and scientists trained to appreciate 
the differing approaches of several different research fields. Chapter 7 addresses the pivotal issue of how the United States, and more specifically the Department of Energy, can address the support issues that the Grand Challenges pose.

Many of the processes that characterize energy flow, capture, production, storage, and transduction occur far from equilibrium; so do most significant biological behaviors, and important processes in molecules, solids, oceans, and atmospheres. Since most of our current understanding of physical and biological systems is based on equilibrium concepts, far-from-equilibrium behaviors are intrinsically and crucially challenging. The combination of significance and energy relevance, of intrinsic scientific knowledge and its application, characterize the Grand Challenge of systems far from equilibrium. Progress in understanding and quantifying these behaviors must be made in order to deal effectively with the energy, climate, materials, biological, and security issues facing humankind. 



\section{ENABLING GRAND CHALLENGE SCIENCE: THE PEOPLE AND TOOLS REQUIRED}

The Grand Challenges are crucial because even partial solutions to them will allow experimental intuition and imagination to be guided by understanding. That intuitive and imaginative advantage will allow the scientific, engineering, and industrial labs of the United States to build new instruments, make new materials, develop new theories and processes, and solve important societal problems. These problems, from energy supply to advanced batteries, from environmental security to harnessing biology for human benefit, from new materials to human resources, are among the most significant facing our country and our civilization, and solutions to them will naturally lead to new industries. These are the scientific, technological, and societal benefits of the research discussed in this report.

The transition from observation science to control science envisaged in the five Grand Challenges requires a three-fold attack: new approaches to training and funding, creation of theories and concepts beyond those we currently possess, and development of instruments that are more precise and more flexible than those used for observation science.

The research we foresee is inherently multidisciplinary and will require sustained efforts over long periods of time before society will reap the benefits. Pushing an individual discipline to its limits often leads to research at the interface where multiple fields come together. Progress will require scientists who are firmly anchored in one or two disciplines and who can also communicate effectively with scientists in all of the disciplines underpinning BES research: condensedmatter and materials physics, chemistry, engineering, biology, and areas of earth and atmospheric sciences. Moreover, while the application of basic energy science to the global problem of energy production and utilization is being enthusiastically embraced by the current generation of undergraduate and graduate students, the most effective solutions to the nation's energy security will require awareness of the social, industrial, and technological context of energy science. For all these reasons, we believe that new approaches to training and funding that can support a sustained effort in multiple areas of science are as crucial as new instrumentation and theory development.

The theoretical challenges described in this report cannot simply be reduced to ever larger computations - in many cases we do not know how to formulate the theory well enough to compute anything! We need an ambitious scientific culture that encourages high-risk, high-reward theoretical exploration focused on such Grand Challenge issues as non-equilibrium phenomena, coherence, correlations and the inter-conversion of energy and information. This will attract the best theoretically inclined scientists to basic energy science and produce the new laws and concepts we need to understand and control matter with precision.

Designing materials to have the properties we want and then directing the synthesis to produce these properties and not others requires the ability to see functionality at the relevant length, time, and energy scales (see Figure 7.1). We must interrogate matter at a level much deeper than the macroscopic average (defined as average behavior of many molecules or domains), so that the properties of individual molecules or microscopic domains of a material can be observed and controlled. We therefore need to develop and disseminate new tools capable of viewing the inner workings of matter-transport, reactivity, fields, excitations, and motion-to answer questions such as "How does the active site of a catalyst change during the course of the reaction?" Or "What are the correlations of the electrons in high-temperature superconductors?" If we are to be capable of making quantum systems work for us, we need new tools that go beyond probing what is there but instead propel us toward the goal of controlling matter through direct manipulation of electrons. 


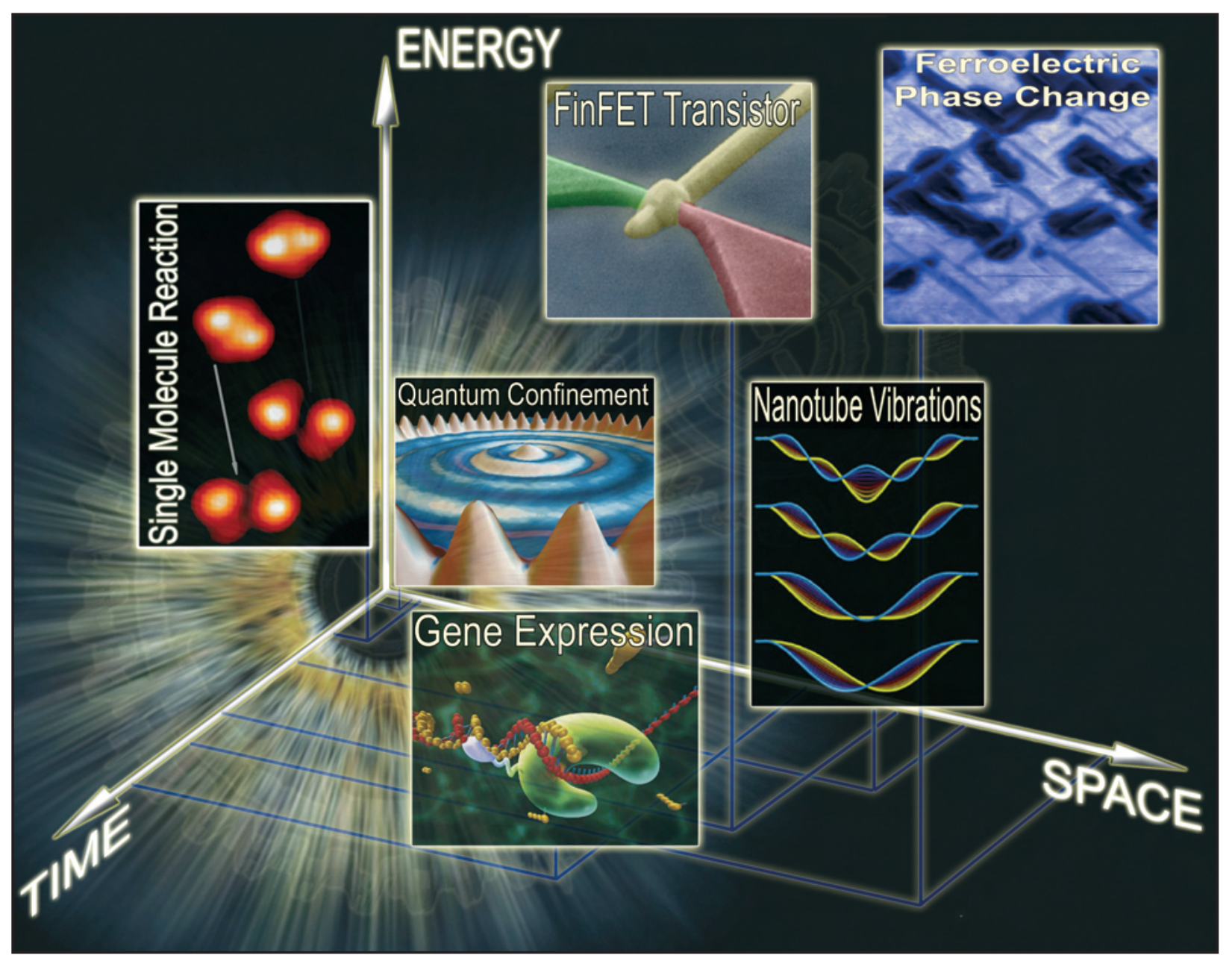

FIGURE 7.1. Designing materials to have the properties we want and then directing the synthesis to produce these properties and not others requires the ability to see functionality at the relevant length, time, and energy scales.

\section{TRAINING AND SUPPORTING THE ENERGY SCIENTISTS OF THE FUTURE}

Attacking the Grand Challenges and providing the United States with the necessary revolutionary ideas to address the overarching national priority of a secure energy future requires fostering, nurturing, and rewarding excellence and creativity in research and in all aspects of education. It is critical to have the right people. The challenges and opportunities discussed in this report call for imaginative and productive new approaches to inspire, train, and support first-rate American scientists. We need new methods to train our next generation of scientists so that they are capable of providing the United States with the deep expertise needed to secure the energy future and transform the nation into the world's undisputed leader in science and in technology. It seems highly likely that sustained efforts over long periods will be required for solutions to the difficult challenges posed in this report (most likely decadesthat is part of why they are Grand Challenges), so that the three-year cycle of grant funding may not always be the most effective method of support.

The most important challenge is to give our future energy scientists, especially graduate students and postdoctoral fellows, the training and support required to succeed in what we are calling interdependent science, where scientists at the forefront of several disciplines come together to create science that is truly more than the sum of the individual contributions. 
Such Grand Challenge science will require sustained support of basic research whose applications are not yet clear.

Given the importance of innovation in basic energy science to the nation, we believe that special efforts to engage and support the most creative and productive scientists and to enhance the prestige of DOE support in this area should be made. Specifically, the committee recommends that DOE provide national leadership (and appropriate funding) towards new capabilitybuilding models that empower the American scientific workforce with the training and the tools for success. Strengthening the U.S. scientific workforce through an increase of its diversity in terms of gender, race, ethnicity, and social class is critical to our nation's success. To assure an American scientific workforce with the knowledge, mastery, and experience to address major research issues in Grand Challenge and energy-security science and their effective transfer to useful applications, we recommend that DOE consider a threepronged approach to capability building and human resources. The three components are:

- A DOE Energy Institute, supporting creativity and accomplishment by the most promising and productive researchers.

- An Energy Sciences Study Group, to approach problems requiring science and technology to meet energy security challenges.

- A DOE Fellows program for training and inspiring young scientists.

\section{DOE Energy Institute}

We propose that some DOE resources be directed towards supporting selected investigators at a significant level. The program we have in mind is modeled after the very successful Howard Hughes Medical Institute Investigator awards. The funding would be unrestricted (but must relate broadly to basic energy science) and be long term. The individual investigators would be called Energy Science Senior Fellows. The selection would be by a blue-ribbon committee consisting of DOE, national laboratory, and university leaders, chaired by a senior investigator. The nature of the Energy Institute Program, plus the funding level and selection process, are designed to provide productivity, diversity, prestige, and visibility.

The DOE Energy Institute would establish a fiveyear program headed by a single principle investigator (PI), whose salary is paid in part by DOE during that time. The support dedicated to such a grant would be considerably greater than current DOE university grants. Energy Science Senior Fellows are expected to push their fields into new areas of enquiry, develop new tools and concepts, and bring in, when necessary, approaches and concepts from other disciplines. The bar for renewal after five years would be set very high. This sort of program would immediately change the prestige and impact of university research sponsored by BES. It would send an unmistakable signal to the university community that a new kind of commitment to basic research has been made, and would provide the freedom necessary for the best scientific minds in the country to focus on truly ground breaking Grand Challenge Science.

\section{Energy Science Study Group}

Following the format of the DARPA-funded Defense Science Study Group, we suggest that the Department of Energy create an Energy Science Study Group (ESSG). The purpose would be to train a group of young scientists to become more fully conversant with rapidly evolving technology. The program would convey to participants both an understanding of the scientific and technical dimensions of national energysecurity issues and an appreciation for the interdisciplinary nature of energy research.

The ESSG would select about 15 young but accomplished scientists per year from a broad geographical range of the nation's top universities, national laboratories, and industry. Over the course of the two-year program, those invited to participate would learn first-hand from visits and in-depth discussions with experts about energy policy, global energy needs, related research and development, and the scientific grand challenges that underpin sustainable energy. The mentors and advisors of the group could come from the U.S. industrial energy sector as well as those areas involving energy and environmental policy. 
During the two year program, the ESSG members would also be responsible for holding a series of international workshops on specific energy needs. This would bring together a multidisciplinary set of scientists and engineers with experts to learn about and think deeply about a particular energy topic, such as the science challenges underpinning carbon sequestration or new, clean, and efficient transportation fuels. These workshops would create Grand Challenge science roadmaps for a particular energy sector (such as transportation fuels or solar power). The ESSG members would then be able to propose small multidisciplinary innovative projects (unconstrained funding for the equivalent of support of one student for three years) in fundamental science or science and engineering that engage energy issues in some way. In this fashion, the ESSG members and their students would be encouraged to be creative and multidisciplinary in their approach to the scientific Grand Challenges underpinning our energy needs.

The ESSG members, after graduating, would continue to take part as ESSG alumni and mentors and principal investigators for DOE Science. In addition, ESSG alumni would be knowledgeable about and able to step up to the challenge of providing the science and technology breakthroughs to create sustainable energy sources and processes for the globe, essential for the energy security of the U.S. They would also provide an important resource for developing and applying the insights attained by Grand Challenge Science.

\section{DOE Fellows Program for Young Scientists}

The core subject training of most graduate programs does not fully prepare students to appreciate and therefore contribute to key issues that limit progress in addressing Grand Challenge science. Likewise, newly graduated $\mathrm{PhDs}$ moving from other disciplines in basic energy sciences may require new, trans-disciplinary training to enable them to contribute rapidly to their new field of study.

Three mechanisms, based on ideas that have been successful elsewhere, may prove helpful in preparing a young and diverse set of scientists to address Grand Challenge science.
(1) Summer Energy Experiences (SEE) program. In this program, DOE would collaborate with universities to sponsor, each summer, several SEE training courses. These would take place on the University campuses or at the national laboratory Nanoscale Science Research Centers, and be open to students, postdoctorals, and more established workers interested in attaining new scientific capabilities. Attendees would be called SEE Fellows. The SEE courses would focus on research approaches to Grand Challenge and energysecurity science; they would also provide focused, collaborative, state-of-the-art research training, utilizing the resources of the hosting university or the Nanoscale Science Research Centers. This coordinated program is modeled after the very successful Cold Spring Harbor courses in the Biological Sciences.

(2) Grand Challenge workshops. We envision workshops with foci in specific areas, and with equal numbers of graduate students/postdoctoral scientists and career scientists present. One possibility is to have a four-day program, in which the first two days are devoted to outlining the fundamental concepts and key issues that will be discussed and debated over the following two days. Only a small subset of the senior researchers will be needed to teach the first two days. For the majority of these researchers, the workshop will be a normal scientific meeting, but with much greater involvement and participation by early stage scientists. This mechanism could have significant impact by encouraging junior scientists to pursue a research career, opening up employment opportunities through their contacts with leading scientists and allowing them to expand their collaboration base. It seems likely that a short series of coherent workshops over, say, a two-tothree year period on a specific Grand Challenge area would be the most effective mechanism.

(3) Network Energy Teams. Team Science has been an essential aspect of some science areas for at least 75 years, but is now becoming increasingly important in areas that have been traditionally driven by single-PI activities. This raises the possibility of training in Network Energy Teams. These would work quite similarly to the European Union Marie Curie Research Training Networks, where several teams propose a joint project, and early stage researchers (those completing 
graduate school or postdocs) spend periods of time in more than one research team. This would allow these early stage scientists to customize (in consultation with their advisor) their research training, be exposed to multiple viewpoints on key scientific issues, and begin their own collaborative group at an early and significant career stage.

\section{THEORY FOR THE GRAND CHALLENGES}

Pasteur famously said that chance favors the prepared mind. The role of theory is to prepare our minds-to formulate questions in a way that makes it productive - to imagine new instruments, measurements, materials, and processes. The Grand Challenges require theorists to discover the new behaviors that govern systems from the nanoscale to the human scale. The search for such laws is as difficult and important as theoretical work in cosmology or particle physics. However, the quest for fundamentally new theories is frequently neglected in favor of simpler extensions of existing theories where the basic concepts are already known. While the latter is certainly less risky, developing a culture that encourages deep exploration in theory as well as experiment is necessary for progress on Grand Challenge science. A healthy and demanding culture of theoretical exploration, focusing on the major issues involved in developing theoretical insights into such Grand Challenge topics as coherence, nonequilibrium, and emergence, will help steer the best theoretically inclined scientists away from other research areas with less connection to experiment, to energy, and to human welfare.

\section{PHYSICAL RESOURCES: SEEING MORE DEEPLY INTO NATURE}

New instruments lie at the heart of discovery. To see something new one must do something new, yet the instruments required to image function and to control matter do not exist and must be invented based on energy science. While many of the new tools will be extensions and combinations of current technologies, such as scanning probes, electron microscopes, synchrotron and laser light sources, etc., others must be based on discoveries not yet foreseen. These instruments must image systems in space, time, and energy with the correct resolution for each of these three dimensions (See Sidebar "Imaging Molecular Functionality"). The ability to image structure and function will inform and direct our efforts to control molecules and materials, and from the new generations of instruments will naturally emerge devices to direct matter at the levels of electrons, atoms, or molecules.

A dedicated and sustained effort to invent and disseminate novel and sophisticated instruments forms the experimental underpinning of the Grand Challenges described in this report. Instrumentation advances can be supported in three main ways:

- New instrument development in individual laboratories or small groups. Conceiving and developing new instruments in individual laboratories can provide unanticipated, transformative ways to create and understand materials, devices, and architectures. They also provide unique opportunities to train and inspire young scientists.

- Major national facilities. The DOE's light sources, neutron scattering facilities, and highthroughput genome screening centers represent an extraordinary success story, enabling an enormous range of science across all the disciplines mentioned in this report. Maintaining and upgrading of this resource base is clearly critical to the broad success of Grand Challenge Science.

- Specific new enabling methodologies. The specific areas of imaging tools, next-generation detectors, and materials-synthesis facilities present special opportunities and are areas in which the United States is starting to lag.

Equipment funding must be appropriately balanced among individual investigators, mid-scale, and largescale facilities. Such balance is necessary for the research discussed in this report to be successful and to transform the capabilities of American science and technology. In some instances, such as recent and current research on laser wake fields, the results of individual instrumentation efforts can contribute strongly to the capabilities of large central facilities. 


\section{IMAGING MOLECULAR FUNCTIONALITY}

\section{Coupling Ultra-Fast and Ultra-Small with Light and Scanning Tunneling Microscopy}

The advent of scanning tunneling microscopy (STM) has opened a gateway to the scientific and aesthetic beauty of the nanoworld, allowing us to visualize objects with spatial resolution far exceeding our natural abilities and that of conventional microscopy. Single molecules can today be observed with sub-molecular resolution, manipulated to assemble complex structures and excited to induce chemical transformation. Yet the biggest challenge lies ahead: to go beyond the frozen picture into the vast and intricate domain of molecular dynamics with the ultimate goal of achieving unprecedented control over functionality on a single-molecule level. The key to such an undertaking is breaking the existing barrier of measurement bandwidth, a barrier that limits us to temporal resolutions about ten orders of magnitude slower than the characteristic femtosecond time-scale of chemical reactions, particularly those involving excited molecular states.

One concept of an "ultimate" measurement of molecular dynamics couples the femtosecond temporal resolution of light excitation to the atomic spatial resolution of a scanning tunneling microscope (Figure 1). The STM tip is used to locally enhance the electric field of the incoming light, thereby selectively producing either a chemical reaction or fluorescence of a single molecule. Recent experiments have demonstrated the feasibility of this concept (Figure 2). In one experiment, fluorescence was excited in a porphyrin molecule with sub-molecular precision by means of tunneling electrons. In another experiment, coupling of photons to the tunneling junction with a magnesium porphine (porphines are the parent chemical compounds for porphyrins) molecule was mapped at the atomic scale.

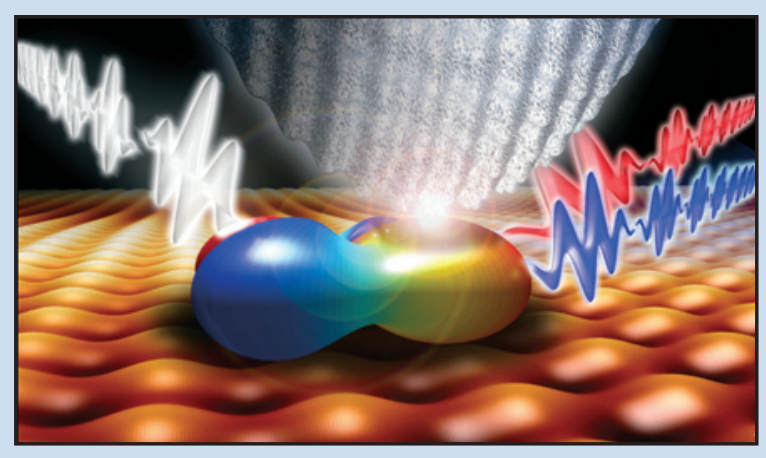

FIGURE 1. Artistic rendition of future single-molecule dynamics measurements. The tip of a scanning tunneling microscope above the molecule locally enhances the electric field of the incident light resulting in red and blue fluorescence.

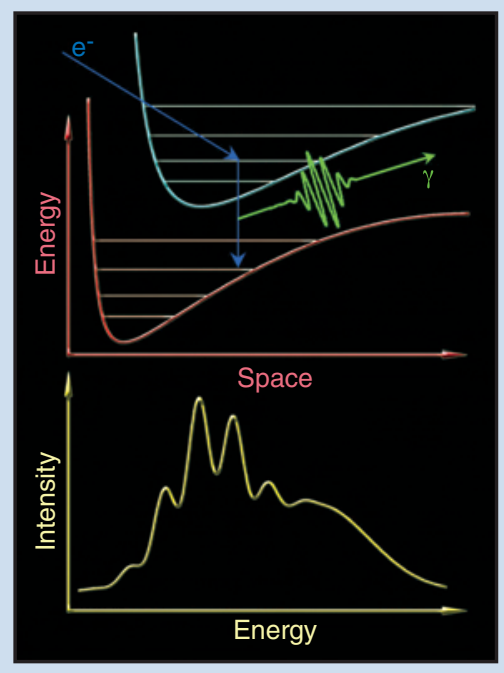

FIGURE 2. Fluorescence of a single porphyrin molecule excited by tunneling electrons with sub-molecular precision. Top: Photons are emitted when the molecule relaxes from the excited (blue) to the ground (red) electronic state. Bottom: The resulting single-molecule fluorescence spectrum. 
We briefly considered what machines or facilities might provide the kind of wide-ranging impact of the current synchrotron facilities or high-throughput genome-sequencing centers. In considering what new facilities are required for Grand Challenge science, we focused on reaching a consensus on the need for certain generic capabilities. Enhancing our current capabilities of time-resolution and imaging arose repeatedly in our discussions. The existing $\mathrm{x}$-ray, neutron, computing, and nanoscience facilities supported by BES will play a major role in Grand Challenge Science, and it will be important to ask each facility to describe how their facility can most effectively respond to the challenges.

There was also consensus that facilities for fabrication of materials, especially crystal synthesis and growth, (see the Sidebar "Crystal-Growth Facilities" in Chapter 4) were lacking. The full information potential of, for example, neutron scattering and angle-resolved photoemission spectroscopy is only reached with large, single-crystal samples.

High-performance computing is also a critical enabler. A few areas in Basic Energy Sciences have made use of the very highest end of the computing spectrum, represented by the most powerful parallel computers in the world. However, much of the progress in the modeling efforts that will be necessary for Grand Challenge science depends on the middle of that spectrum, which is now at the terascale ( $10^{12}$ operations per second). Plentiful and easy access to computing at this level, possible both on large clusters in individual research groups and at centralized computing user facilities, is essential to the development of the new modeling languages and capabilities that will address the Grand Challenges. To create understanding through multi-scale simulations in extended nanoscale and biological systems, computing at the petascale and beyond will be needed.

For major instrumentation needs, we consider that further study and input from the scientific community is required to determine the best path forward. We recommend that the community be engaged via a BESAC subcommittee charged with the responsibility to make specific recommendations for implementing Grand Challenge science. This input should be sought as quickly as possible in order for the U.S. to remain internationally competitive.

\section{New Ways of Probing and Controlling Matter}

The coherent interaction between light and matter lies at the heart of control at the level of quantum mechanics (see Chapter 2). We have very far to go in the quest for the "wave synthesizer" mentioned in Chapter 2 that connects matter waves and electromagnetic radiation in a phase coherent manner, but it is clear that improved time resolution from the ultraviolet through the soft to the hard $\mathrm{x}$-ray regions is an essential component of Grand Challenge science.

There are both laser-based and accelerator-based approaches to providing this capability. The Linac Coherent Light Source (LCLS) under construction at Stanford Linear Accelerator is designed to produce ultra-short ( 1 to $230 \mathrm{fs}$ ) duration $\mathrm{x}$-ray pulses with wavelengths between 1.5 and $15 \AA$ ( $8 \mathrm{keV}$ to $800 \mathrm{eV}$ ). It is scheduled for completion in 2009 with full operation in 2010.

Imaging materials and devices with atomic resolution, chemical specificity, and electronic and magnetic state characterization will also be essential for meeting many of the challenges proposed in this report. Aberration-corrected scanning transmission electron microscopes currently under development will have $0.05-\mathrm{nm}$ spatial resolution for real-time imaging and for direct imaging of individual nanostructures and of reaction dynamics and mechanisms. The National Synchrotron Light Source II (NSLS II) at Brookhaven National Laboratory will provide extremely high brightness and flux with about 1-nm spatial resolution, about $0.1-\mathrm{meV}$ energy resolution, and single-atom sensitivity. NSLS II is scheduled to begin initial operations in 2013.

Complementing these light sources is the Spallation Neutron Source (SNS) at Oak Ridge National Laboratory. This new pulsed source will greatly expand capabilities for scattering studies and allow, for example, time-resolved (on the seconds to minutes timescale) structural studies of materials as they operate in devices or are subject to external stresses.

(a) Next-generation light sources. Synchrotron storage rings for the production of ultraviolet and $\mathrm{x}$-ray light have reached a high degree of refinement. New types of light sources with much shorter pulses and much larger flux and brightness than is currently 


\section{FOUR GENERATIONS OF LIGHT SOURCES}

The First Three Generations. Electron synchrotrons have evolved through three generations to provide highly optimized x-ray sources for diffraction, imaging, and spectroscopy. They are based on electron storage rings around which electrons travel in "bunches" of tens to a few hundred picoseconds in duration. When forced to follow a curved path through bending magnets or directed through a linear array of periodic magnets (known as insertion devices, which come in two flavors - undulators and wigglers ), the electrons emit light pulses of duration equal to that of the electron bunches. The Advanced Light Source (ALS) at Lawrence Berkeley National Laboratory, the Advanced Photon Source (APS) at Argonne National Laboratory, and the Stanford Synchrotron Radiation Laboratory (SSRL) are all third generation sources. The National Synchrotron Light Source II (NSLS-II) at Brookhaven National Laboratory will be a green-field, third-generation replacement for the original NSLS, with enhanced capabilities, particularly brightness, that push storage-ring technology to its limit.

Fourth-Generation. Interrogating atoms, molecules and materials with coherent light pulses on the timescale of the electron motion is a critical first step toward control at the quantum level (see Chapter 2). For such experiments, a light source is needed with all of the properties of a laser beam but with photon energies tunable from VUV into the $\mathrm{x}$-ray region and pulse durations from picoseconds to attoseconds. A light source with the following properties would create great opportunities:

- Even higher flux, brightness, peak power (power per pulse), average power, and stability than available with the third-generation synchrotron radiation sources (Figure 1).

- Much shorter pulse widths and hence temporal resolution.

- Temporal and spatial coherence.

Providing these properties requires redesigning the familiar synchrotron storage ring to capture some of the emittance advantages of a linear accelerator (linac). Energy-recovery linacs (ERL) and free-electron lasers (FEL) are first attempts in this direction, and more novel designs that combine laser and accelerator technology appear likely in the future. No single machine will optimize all of the improvements listed above. For example, high-peak-power machines

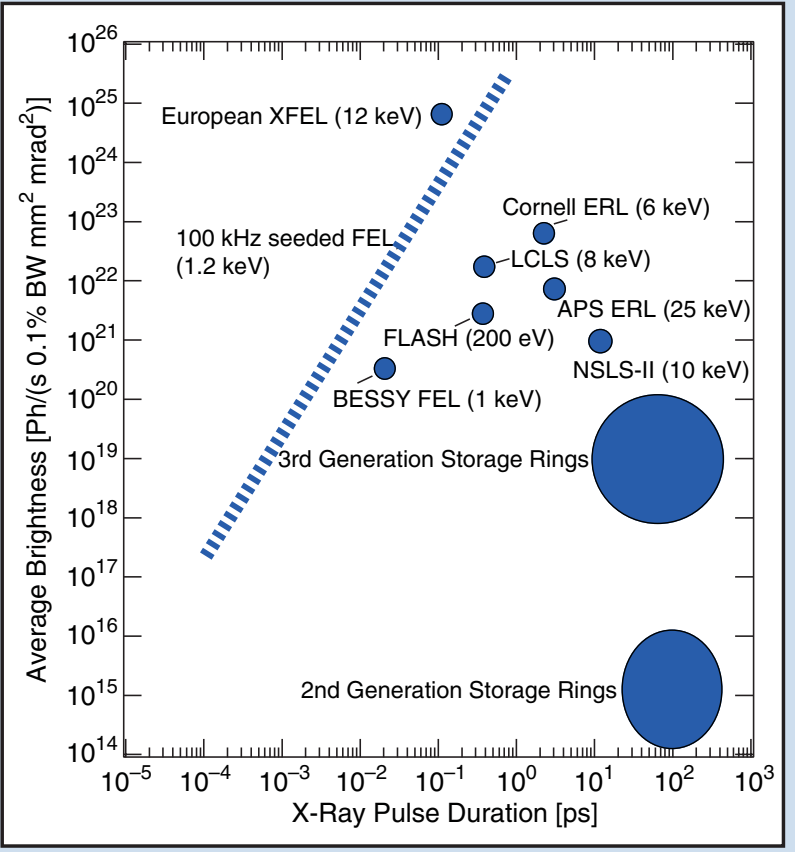
may not provide the best stability and average power.

In the United States, the Linac Coherent Light Source (LCLS) at the Stanford Linear Accelerator Center is being built as an $\mathrm{x}$-ray laser at a wavelength of $1.5 \AA$. The Thomas Jefferson National Laboratory operates an ERL that drives an infrared FEL at a repetition rate up to tens of megahertz. Efforts to extend performance of FELs and ERLs are being actively pursued around the world.

FIGURE 1. Average brightness as a function of pulse duration for representative storage-ring, ERL, and FEL sources in the VUV to $x$-ray range. For identified proposals, values are shown for design brightness at the photon energies indicated in parentheses, based on available data. 
available are now being proposed and constructed. This "next generation" of light sources (see Sidebar "Four Generations of Light Sources") is based either on freeelectron lasers (FELs), energy-recovery linacs (ERLs), or sometimes a combination of the two. For example, in Europe and Asia, at least 13 facilities are being proposed or under construction to produce ultrashort pulses in the UV and $\mathrm{x}$-ray ranges, almost all of which are based on these approaches. Regarding our proposal for a BESAC subcommittee to investigate major instrumental needs, we urge that, for light sources, both the accelerator and potential user communities participate, so that the design space capable of providing the maximum scientific payoff can be defined.

As a complement to conventional accelerator-based light sources, very significant advances in the development of high-power laser systems have occurred in recent years. Such sources are already valuable for the first exploration of attosecond physics and the generation of intense $\mathrm{THz}$ radiation. They have also produced a $1-\mathrm{GeV}$ electron beam via optical acceleration. We consider that an evaluation of the U.S. portfolio in this area would be very timely in conjunction with the BESAC subcommittee study proposed above for major instrumentation needs.

(b) New imaging tools. The transition to control science requires us to visualize and manipulate function and mechanism at the atomic level. From the structure of DNA to that of a moving surface step, an emerging dislocation (nucleating crystal growth), or an array of superconductor vortices, no technique has provided greater confidence in theoretical ideas (or suggested new ones) more effectively than imaging at the nearatomic scale. In each case, mechanisms, function, and dynamics were suggested by a single image. But we need to go further and learn how to image and manipulate with molecular resolution those properties that directly lead to function and change, such as the electric field in the active site of an enzyme, the energy flows in a nanomachine, or the arrangement of spins in a device. Radically new instruments will result from combining techniques from different fields, and such interdependent instrument science should be strongly encouraged.
High-resolution and time-resolved imaging and spatially resolved spectroscopy (see Figures 7.2 and 7.3 and Sidebar "Imaging Molecular Functionality") provide the key to the "reduction of mechanisms to chemistry" needed to understand most of our grand challenges at the atomic level. Spectacular recent advances in the attainment of resolution beyond the diffraction limit, femtosecond imaging, lensless imaging, atomic manipulation at surfaces, tagging methods for new optical microscopies, chemically sensitive photoemission images, and the formation of electron probes of sub-Angstrom dimensions for imaging and spectroscopy hint at what may be possible. In-situ imaging (e.g., during synthesis) has allowed us to monitor the fabrication of new structures almost at the atomic scale in real time. In light of the theoretical difficulties in predicting pathways, this capability is in urgent need of development. Active-site identification in catalysis by atomic-scale imaging remains an elusive and important goal, as well as the atomic-scale imaging and spectroscopy of defects, which can reveal, for example, the mechanism by which hydrogen degrades solar cells and

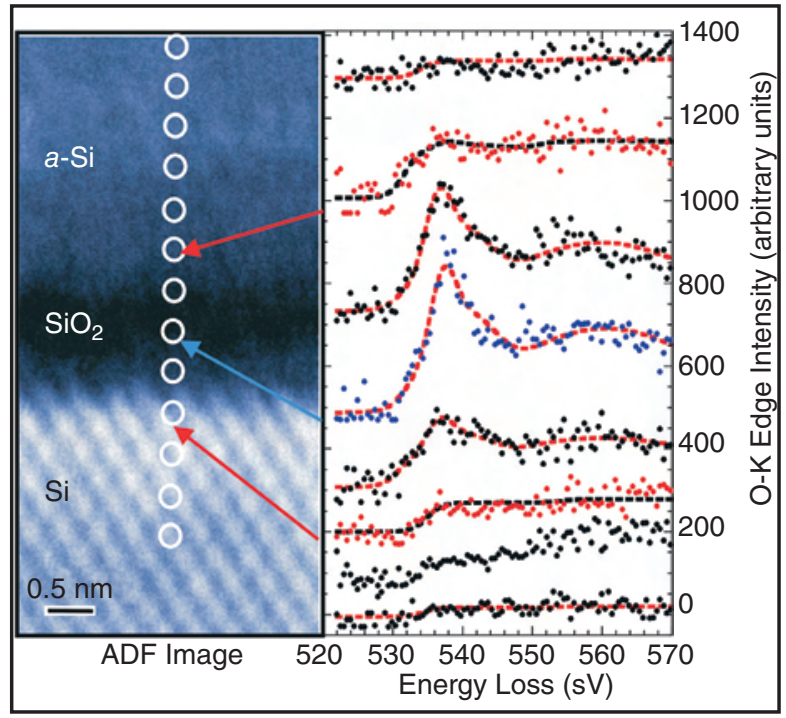

FIGURE 7.2. An electron-energy-loss spectrum (EELS) recorded point by point across a gate stack containing a thin gate oxide (the narrowest feature in today's integrated circuits). Left: Circles in the annular dark field (ADF) image show where each spectrum was taken. The [100] silicon substrate is in the lower half; the gate oxide is in the middle; and the deposited a-Si layer in the top half. Right: The background-corrected oxygen $\mathrm{K}$ edges for the various positions across the gate stack. 


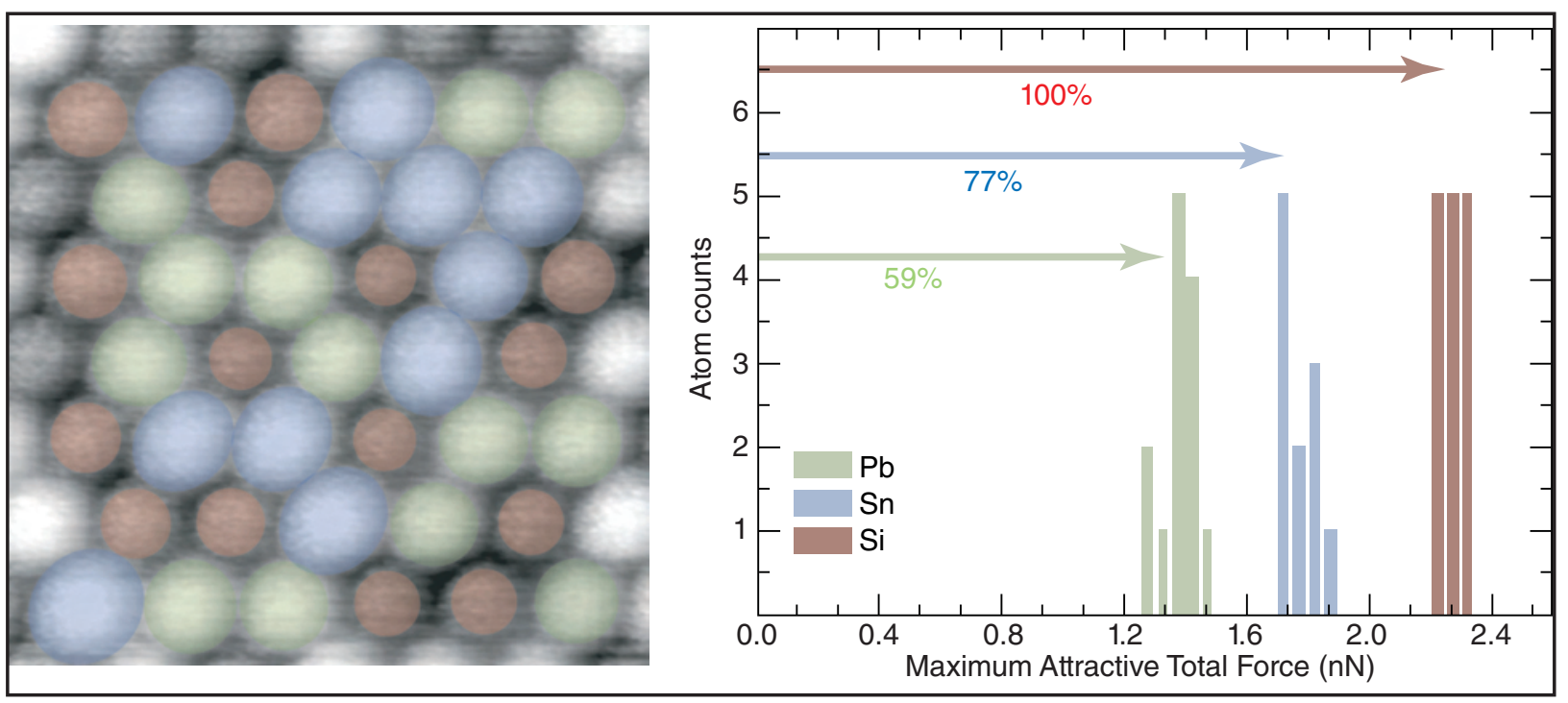

FIGURE 7.3. Left: Single-atom chemical identification by scanning dynamic force microscopy at the surface of a tin-lead-silicon $(\mathrm{SnPbSi})$ alloy (blue-Sn, green-Pb, red-Si). Right: Distribution of maximum attractive total forces measured over the atoms in the image.

fuel-cell materials. The ability to form lensless images using a single $\mathrm{x}$-ray pulse shorter than the nuclear vibration period has already been demonstrated, presaging the creation of real-time movies of molecular dynamics.

(c) Next-generation detectors. The detectors used today across most of the physical and biological sciences are based on commercial technologies or modifications thereof. This is in stark contrast to high-energy physics, where the experimental demands of separation of very rare collision products from an enormous competing background necessitated the early development of highly parallel detectors with intrinsic processing and decision-making power.

Detectors that are fully parallel with pixel-level processing and are capable of decision-making export processed data rather than raw data, and they have the potential to revolutionize many types of experiments. For example, in x-ray photon correlation spectroscopy (XPCS), the spatial and time dependence of a process is monitored through measurement of the temporal autocorrelation spectrum over a range of spatial frequencies via $\mathrm{x}$-ray diffraction. Present-day technologies limit the technique to either single spatial frequencies with nanosecond temporal resolution or a large array of spatial frequencies with a two-dimensional detector but with a temporal resolution of a few milliseconds.

If we apply and adapt the technologies of high-energy physics detectors to this area, the result would be revolutionary. For example, both ultrafast and high-resolution tomographic imaging science would be greatly aided by improvements and new features in detector capability. In another example, the properties of complex materials are guided by fluctuations in electronic and structural configurations, from a biological system seeking a potential energy minimum through structural deformation to electronic fluctuations of spin, charge, and orbital ordering in correlated electronic systems. $\mathrm{X}$-ray correlation spectroscopy with advanced detectors would give access to length scales from the atomic to the macroscopic, and from slow dynamics all the way to the picosecond regime.

Similar comments can be made with regard to dynamic light microscopy, where large pixellated arrays of sensors with gain can be developed and combined with CMOS pixel processing, and to dynamic electron microscopy, where there is now an urgent need to develop very large array detectors with excellent point spread 
function response for high-energy electrons and with fast framing and time stamping capabilities. This will enable the "movies" of dynamical phenomena mentioned above and take electron microscopy from the domain of observation of static phenomena or slow dynamics to the nanosecond domain and beyond.

\section{Creating New Materials}

The creation of new materials forms an essential component of the Grand Challenges. Here, our committee formed a strong consensus that new facilities for the growth of novel crystals are a key requirement for rapid progress. This is an area (perhaps more than any other) that has suffered from the decline of the great industrial research laboratories in the United States. The most useful and exotic condensed-matter systems-superconductors, semiconductors, magnets, ferroelectrics-are usually crystalline matter. Crystalline systems will likely comprise fundamental components of quantum information processors, efficient solar cells, or novel sensors for multiple applications. They provide a platform for truly novel states of matter such as quantum liquids, spin ice, and composite fermions.

There are two areas where we see significant needs and opportunities. At present, there is a mismatch between the supply and demand of crystalline materials, so that the infrastructure for existing types of synthesis needs to be expanded and renewed. Second, capabilities to synthesize crystals of toxic or radioactive materials and materials created at extreme pressures and/or temperatures and to synthesize very large single crystals are needed (see the Sidebar "Crystal Growth Facilities" in Chapter 4). Materials to be gained from these more specialized and expensive facilities include fluorides for topological spin matter, phthalates for negative-pressure superconductors and magnets, and oxides for $\mathrm{THz}$ frequency conversion.

Such facilities should be complemented by an organizing center for crystal synthesis that would be responsible for an advanced materials database, coordinate researchers' requests for samples, and provide education and training in crystal synthesis.

Progress in catalytic science would greatly benefit from a nationwide multi-user facility for highthroughput generation and evaluation of heterogeneous and homogeneous catalysts. This would allow rapid, efficient synthesis of substantial catalyst libraries and screening of the resulting structures for activity and selectivity in specified catalytic transformations. Such specialized facilities are heavily utilized in industrial laboratories but are not generally accessible to the academic or national laboratory communities. A highthroughput catalyst-discovery facility would be most productive when coupled to one of the aforementioned materials analysis and imaging facilities.

\section{CONCLUSIONS}

In order to take advantage of the extraordinary opportunities described in this report, Basic Energy Sciences will need to build and maintain the following world leading capabilities for the future:

- A highly trained, diverse, and empowered scientific workforce that possesses scientific depth in one or two areas and is able to collaborate across a breadth of scientific and technical fields.

- A group of theorists, concentrating on the very difficult and demanding fundamental questions that arise in such problems as systems far away from equilibrium or nanoscale communication or strong correlations.

- Appropriate new experimental and computational facilities and a balance of funding mechanisms permitting development of new instruments, tools, and materials capabilities for single PIs, team science, and major user facilities. Such capabilities will transform basic energy sciences, play a critical role in securing our energy future, and produce applications not yet imagined. 



\section{APPENDIX 1}

\section{THE GRAND CHALLENGES SUBCOMMITTEE PROCESS}

The formal origin of the Grand Challenges study is a January 25, 2005 request from DOE Office of Science Director, Ray Orbach, to BESAC Chairperson John Hemminger (University of California, Irvine) to hold a workshop to examine the key scientific questions for the Basic Energy Sciences with the following goals:

1. Identify and articulate for the broader scientific community the most important scientific questions and science-driven technical challenges facing the disciplines supported by BES.

2. Describe the importance of these challenges to advances in disciplinary science, to technology development, and to energy and other societal needs.

3. Describe what insight might be needed to address these challenges, including the development of theories, instruments, facilities, and computational capabilities and education and workforce development.

4. Connect the challenges with disciplines outside those supported by BES, as appropriate.

5. Use as resource material prevous discussions at BESAC and relevant studies by BESAC, other SC Advisory Committees, the NRC, and other bodies.

6. Suggest follow-on activities, as appropriate.

To permit the extended study needed to respond to this request, rather than initiating a workshop, BESAC formed a Subcommittee on Grand Scientific Challenges for the Basic Energy Sciences with Graham Fleming (Lawrence Berkeley National Laboratory and University of California, Berkeley) and Mark Ratner (Northwestern University) as Co-Chairs. The subcommittee held three brainstorming sessions, in Berkeley, CA (July 2006), Evanston, IL (October 2006), and Berkeley, CA (April 2007), before producing a draft report for presentation at the July 31-August 1, 2007 BESAC meeting. Weekly teleconferences were also held from April 2007 onward. A subsequent brain- storming session immediately after the BESAC meeting at Rockville, MD (August 2007) and two editorial meetings in Boston, MA (August 2007) and Berkeley, CA (August 2007) were held prior to producing a second draft for approval at the September 20, 2007 BESAC meeting. The final report includes further refinements in response to BESAC recommendations.

In order to define Grand Challenges for the Basic Energy Sciences, the subcommittee set the following criteria:

A Grand Challenge must

- Be scientifically deep and demanding.

- Be clear and well defined.

- Be relevant to the broad portfolio of BES.

- Promise real dividends in devices or methods that can significantly improve the quality of life and help provide a secure energy future for the United States.

The next task was to ask each subcommittee member and some members of BESAC to fill out a survey that asked the following questions:

1. What is/are your Big Question(s)? Please create one-to-three such questions and state each in one sentence.

2. What are the issues surrounding your Big Question? Please describe in one paragraph (250 words or less) for a non-specialist audience.

3. Please provide a full description of your Big Question and include 1) its relevance to other fields and 2) its relevance to BES and DOE (BES Mission statement is appended):

4. Is there science infrastructure (including workforce issues) that needs to be developed to address this Big Question? Please describe.

5. Describe any specialized funding mechanisms that could be useful or necessary to address this Big Question. 
There was no limit to the number of responses any subcommittee member could provide. Around one hundred surveys were completed, and from these a number of scientific areas emerged where it was clear that our current knowledge and capability were seriously deficient.

The areas were:

1. When we go to the very small.

2. When we go far from equilibrium.

3. When we encounter strongly correlated systems and systems with emergent properties.

4. When we want to define the limits of material properties.

5. When we want to manipulate energy and information ever more rapidly and efficiently.

6. When we want to recreate in synthetic systems properties and capabilities we find in nature.

Underlying these areas a set of concepts emerged (see Figure 1.1 in Chapter 1).
The subcommittee discussions and its broader consultation of the scientific community suggested that we are on the threshold of a transition from observation science to control science at a much deeper level than is currently possible. Combining this notion with the set of underlying concepts in Figure 1.1 led the subcommittee to identify the five broad Grand Challenge areas that are described in Chapters 2 through 6.

A primary writer from among the subcommittee members was assigned to each science chapter (and in the case of Chapters 4 and 5, secondary writers were also added). The authors are: Chapter 2, Philip Bucksbaum (Stanford University and Stanford Linear Accelerator Center); Chapter 3, Tobin Marks (Northwestern University); Chapter 4, Leon Balents (University of California, Santa Barbara), Joel Moore (Lawrence Berkeley National Laboratory and University of California, Berkeley), and Julia Phillips (Sandia National Laboratories); Chapter 5, Jay Groves (Lawrence Berkeley National Laboratory and University of California, Berkeley) and Paul McEuen (Cornell University); Chapter 6, Mark Ratner (Northwestern University); and Chapter 7, Graham Fleming (Lawrence Berkeley National Laboratory and University of California, Berkeley). 


\section{ACKNOWLEDGEMENTS, FIGURE SOURCES, AND RESOURCE MATERIALS}

\section{FROM THE GRAND CHALLENGES SUBCOMMITTEE CO-CHAIRS}

The Co-chairs Graham Fleming and Mark Ratner would like to extend a special thank you to the following individuals whose assistance and expertise were invaluable to the making of this report: Adam Arkin (Lawrence Berkeley National Laboratory and University of California, Berkeley), Kristin Balder-Froid (Lawrence Berkeley National Laboratory), Jim Barber (Imperial College London), Roberto Bassi (University of Verona), Kyle Bishop (Northwestern University), Arup Chakraborty (Massachusetts Institute of Technology), John Corlett (Lawrence Berkeley National Laboratory), Gavin Crooks (Lawrence Berkeley National Laboratory), Peter Denes (Lawrence Berkeley National Laboratory), Phillip Geissler (Lawrence Berkeley National Laboratory and University of California, Berkeley), Bartosz Grzybowski (Northwestern University), John Hemminger (University of California, Irvine), Nancy Holt (SAIC), Walter Kohn (University of California, Santa Barbara), Joel Lebowitz (Rutgers University), Karin Levy (Lawrence Berkeley National Laboratory), Steve Louie (Lawrence Berkeley National Laboratory and University of California, Berkeley), Jan Liphardt (Lawrence Berkeley National Laboratory and University of California, Berkeley), Luke Lee (University of California, Berkeley), Shaul Mukamel (University of California, Irvine), Peter Nixon (Imperial College London), George Oster (University of California, Berkeley), Howard Padmore (Lawrence Berkeley National Laboratory), Ward Plummer (University of Tennessee), Arthur Penn Ramirez (Bell Labs, Alcatel-Lucent), Stuart Rice (University of Chicago), Arthur Robinson (Lawrence Berkeley National Laboratory), Robert Schoenlein (Lawrence Berkeley National Laboratory), Gary Snider (Hewlett-Packard), Josh Vura-Weis (Northwestern University), and Lynn Yarris (Lawrence Berkeley National Laboratory).
They also thank, particularly and gratefully, Tiffaney Dressen - without Tiff, this report would be later and less useful.

\section{CHAPTER 2}

\section{Authors and Contributors}

This chapter was coordinated by subcommittee member Phil Bucksbaum. Additional contributions were received from many individuals, including: BESAC member Bill McCurdy (Lawrence Berkeley National Laboratory and University of California, Davis), subcommittee members Walter Kohn (University of California, Santa Barbara), Cherry Murray (Lawrence Livermore National Laboratory), and Jun Ye (University of Colorado, Boulder, and JILA), as well as Philip Anderson (Princeton University), Joe Eberly (University of Rochester), Brett Esry (Kansas State University), Matthew Fisher (University of California, Santa Barbara and Microsoft), Pierre Meystre (University of Arizona), David Reis (University of Michigan), Frank Wilczek (Massachusetts Institute of Technology), Linda Young (Argonne National Laboratory), and many others.

\section{CHAPTER 3}

\section{Authors and Contributors}

This chapter was coordinated by Tobin Marks and received input from many individuals, including Mark Barteau (University of Delaware), Alex Bell (University of California, Berkeley and Lawrence Berkeley National Laboratory), John Bercaw (California Institute of Technology), Bill Banholzer (Dow Chemical), George Crabtree (Argonne National Laboratory), Frank DiSalvo (Cornell University), Arthur Epstein (Ohio State University), Graham Fleming (Lawrence 
Berkeley National Laboratory and University of California, Berkeley), John Hemminger (University of California, Irvine), Mark Hersam (Northwestern University), Mercouri Kanatzidis (Northwestern University), Walter Kohn (University of California, Santa Barbara), Gabrielle Long (Argonne National Laboratory), Bill McCurdy (Lawrence Berkeley National Laboratory and University of California, Davis), Joel Miller (University of Utah), Martin Moskovits (University of California, Santa Barbara), Matt Neurock (University of Virginia), Dan Nocera (Massachusetts Institute of Technology), Teri Odom (Northwestern University), Ward Plummer (University of Tennessee), Mark Ratner (Northwestern University), John (Jack) Richards (California Institute of Technology), Gabor Somorjai (Lawrence Berkeley National Laboratory and University of California, Berkeley), John Spence (Arizona State University), Mary Wirth (University of Arizona), and many others.

\section{CHAPTER 4}

\section{Authors and Contributors}

This chapter was coordinated by Leon Balents, Joel Moore, and Julia Phillips. We would like to thank James Langer (University of California, Santa Barbara), Douglas Scalapino (University of California, Santa Barbara), Eric Isaacs (Argonne National Laboratory), Laura Greene (University of Illinois, Urbana-Champaign), Ramamoorthy Ramesh, (University of California, Berkeley, and Lawrence Berkeley National Laboratory), and especially Walter Kohn (University of California, Santa Barbara) for key inputs into this chapter.

\section{CHAPTER 5}

\section{Authors and Contributors}

This chapter was coordinated by Jay T. Groves and Paul L. McEuen. Valuable input was received from many individuals, including: Adam Arkin (University of California, Berkeley, and Lawrence Berkeley
National Laboratory), Arup Chakraborty (Massachusetts Institute of Technology), Eric Isaacs (Argonne National Laboratory), Walter Kohn (University of California, Santa Barbara), Bill McCurdy (Lawrence Berkeley National Laboratory and University of California, Davis), Cherry Murray (Lawrence Livermore National Laboratory), George Oster (University of California, Berkeley), John (Jack) Richards (California Institute of Technology), John Spence (Arizona State University), Stan Williams (Hewlett-Packard), and many others.

\section{CHAPTER 6}

\section{Authors and Contributors}

This chapter was coordinated by subcommittee co-chair Mark Ratner. Additional contributions were received from many individuals, including: Stephen Berry (University of Chicago), Kyle Bishop (Northwestern University), David Chandler (University of California, Berkeley and Lawrence Berkeley National Laboratory), Gavin Crooks (Lawrence Berkeley National Laboratory), Irving Epstein (Brandeis University), Bartosz Grzybowski (Northwestern University), Leo Kadanoff (University of Chicago), Joel Lebowitz (Rutgers University), Jan Liphart (Lawrence Berkeley National Laboratory and University of California, Berkeley), Phillip Geissler (Lawrence Berkeley National Laboratory and University of California, Berkeley), Stuart Rice (University of Chicago), and Josh Vura-Weis (Northwestern University).

\section{CHAPTER 1}

\section{Figure Sources}

Figure 1.1. From the Grand Challenges subcommittee, BESAC.

Figure 1.2. Please see figure source for Chapter 2, Figure 2.1.

Figure 1.3. Please see figure source for Chapter 3, Figure 3.6. 
Figure 1.4. Please see figure source for Chapter 4, Figure 4.1.

Figure 1.5. Please see figure source for Chapter 5, Figure 5.6.

Figure 1.6. Courtesy of Eshel Ben Jacob (Tel Aviv University); http://star.tau.ac.i1/ eshel/

Figure 1.7. Photograph by Roy Kaltschmidt (Lawrence Berkeley National Laboratory, CSO); Courtesy of Fleming Research Group members Hohjai Lee, Gabriela Schlau-Cohen and Naomi Ginsberg, (Lawrence Berkeley National Laboratory and University of California, Berkeley).

Figure 1.8. Reprinted with permission from E.T. Foley, N.L. Yoder, N.P. Guisinger, and M.C. Hersam, Rev. Sci. Instrum., 75, 5280 (2004); Copyright 2004, American Institute of Physics.

\section{CHAPTER 2}

\section{Figure Sources}

Figure 2.1. Reprinted Figure 1 (inset) with permission from L.P. Rokhinson, V. Larkina, Y.B. Lyanda-Geller, L.N. Pfeiffer, and K.W.West, Phys Rev Lett., 93(14), 146601 (2004); Copyright 2004, by the American Physical Society.

Figure 2.2. M. Ben-Nun, F. Molnar, H. Lu, J.C. Phillips, T.J. Martínez, and K. Schulten, Faraday Discussions, 110, 447 (1198);

Reproduced by permission of The Royal Society of Chemistry.

Figure 2.3. Reprinted by permission from Macmillan Publishers Ltd: Nature, R.J. Sension, Nature, 446, 740 (2007).

Figure 2.4. Courtesy of Shinkoh Nanbu (Kyushu University); http://sn-gate.cc.kyushuu.ac.jp/sb/

Figure 2.5. Plasma Science: Advancing Knowledge in the National Interest, Plasma 2010 Committee, National Research Council,
ISBN: 0-309-10940-X; Reprinted with permission from the National Academies Press, Copyright (2007), National Academy of Sciences; http://www.nap.edu/ catalog.php?record_id $=11960$

Figure 2.6. Courtesy of Andre Schirotzeck (Massachusetts Institute of Technology); http://cua.mit.edu/ketterle_group/ experimental_setup/BEC_I/image_ gallery.html

Figure 2.7. Reprinted by permission from Macmillan Publishers Ltd: Nature, Y. Silberberg, Nature, 430, 624 (2004).

Figure 2.8. Reprinted with permission from H.C. Kapteyn, M.M. Murnane, and I.P. Christov, Physics Today, 58(3), 39 (2005); Copyright 2005, American Institute of Physics.

Figure 2.9. Reprinted by permission from Macmillan Publishers Ltd: Nature, M. Drescher, M. Hentschel, R. Kienberger, M. Uiberacker, V. Yakovlev, A. Scrinzi, Th. Westerwalbesloh, U. Kleineberg, U. Heinzmann and F. Krausz, Nature, 419, 803 (2002).

Figure 2.10. Courtesy of Gordon Moore (Intel); http://www.intel.com/technology/moores law/index.htm).

\section{Sidebar "Theoretical Challenges of Quantum} Calculations"

Figure 1. (left) From T.N. Rescigno, M. Baertschy, W.A. Isaacs, and C.W. McCurdy, Science, 286, 2474 (1999); Reprinted with permission from AAAS. (right) W. Vanroose, D.A. Horner, F.Martín, T.N. Rescigno, and C.W. McCurdy, Phys. Rev. A, 74, 052702 (2006); Copyright 2006 by the American Physical Society.

\section{Sidebar "Entanglement"}

Figure 1. AMO 2010: Controlling the Quantum World, The Science of Atoms, Molecules, and Photons, National Research Council, 
2007; Reprinted with permission from the

National Academies Press, Copyright

2007, National Academy of Sciences.

\section{Sidebar "Conical Intersections and Wavepacket} Dynamics

Figure 1. G.A. Worth and L.S. Cederbaum, Annu.

Rev. Phys. Chem. 55, 127 (2004).

Reprinted with permission from the Annual Review of Physical Chemistry, 55;

Copyright 2004 by Annual Reviews, www.annualreviews.org

\section{Sidebar "Quarks"}

Figure 1. Reprinted by permission from Macmillan Publishers Ltd: Nature Physics, F. Wilczek, Nature Physics, 3, 375 (2007).

\section{Sidebar "Quantum Electron Scattering"}

Figure 1. AMO 2010: Controlling the Quantum World: The Science of Atoms, Molecules, and Photons, National Research Council, 2007; Reprinted with permission from the $\mathrm{Na}$ tional Academies Press, Copyright 2007, National Academy of Sciences; Original artwork by D. Villeneuve, NRC. Ottawa.

\section{CHAPTER 3}

\section{Figure Sources}

Figure 3.1. Stock image

Figure 3.2. Courtesy of Nanosys; http://www.nanosysinc.com/

Figure 3.3. Reprinted by permission from Macmillan Publishers Ltd: Nature Materials, K.S. Toohey, N.R. Sottos, J.A. Lewis, J.S. Moore, and S.R. White, Nature Materials, 6, 581 (2007).

Figure 3.4. G.S. Chen, J.A. Labinger and J.E. Bercaw, PNAS 104, 6915 (2007); Copyright (2007) National Academy of Sciences, U.S.A.
Figure 3.5. Courtesy of Yoshinori Naruta (Institute for Materials Chemistry and Engineering, Kyushu University); http:// narutalab.ifoc.kyushuu.ac.jp/index_e.html

Figure 3.6. Courtesy of Universal Display Corporation; http://www.universaldisplay.com/

Figure 3.7. From J. Aizenberg, J.C. Weaver, M.S. Thanawala, V.C. Sundar, D.E. Morse and P. Fratzl, Science, 309, 275 (2005); Reprinted with permission from AAAS.

Figure 3.8. Reprinted with permission from R.W. Armstrong, J.-M.Beau, S.-H. Cheon, W.J. Christ, H.Fujioka, W.-H. Ham, L.D. Hawkins, H. Jin, S.-H. Kang, Y.Kishi, M.J. Martinelli, W.W. McWhorter, Jr., M. Mizuno, M. Nakata, A.E. Stutz, F.X.Talamas, M.Taniguchi, J.A. Tino, K. Ueda, J. Uenishi, J.B. White, and M.Yonaga, J. Am. Chem. Soc., 111, 7530 (1989); Copyright 1989, American Chemical Society.

Figure 3.9. Courtesy of Matt Neurock (University of Virginia), Michael Janik (Penn State), R.J. Davis (University of Virginia), and Rutger A. van Santen (Eindhoven University of Technology)

Figure 3.10. Stock image

Figure 3.11. http://en.wikivisual.com/images/9/9b/ Transrapid.jpg

Figure 3.12. G. Somorjai, R. York, D. Butcher and J. Park, Phys. Chem. Chem. Phys., 9, 3500 (2007); Reproduced by permission of the PCCP Owner Societies.

Figure 3.13. Courtesy of Sandra Rosenthal and her research group (Vanderbilt University); http://www.vanderbilt.edu/AnS/ Chemistry/groups/rosenthal/

Figure 3.14. Reprinted by permission from Macmillan Publishers Ltd: Nature Nanotechnology, M.S. Arnold, A.A. Green, J.F. Hulvat, S.I. Stupp, and M.C. Hersam, Nature Nanotechnology, 1, 60 (2006); Copyright 2006. 
Figure 3.15. Courtesy of Harry Atwater and his research group (California Institute of Technology); "Guiding Light" by $\mathrm{H}$. Atwater, oeMagazine, 2(7) 42 (2002); Reprinted with permission from The International Society for Optical Engineering.

Figure 3.16. From D. Schurig, J.J. Mock, B.J. Justive, S.A. Cummer, J.B. Pendry, A.F. Starr and D. R. Smith, Science, 314, 977 (2006); Reprinted with permission from AAAS.

Figure 3.17. Courtesy of Google images.

\section{Sidebar "Nature's Answer to Fragile Building Blocks"}

Figure 1. (Top) created by Roberto Bassi (University of Verona) Published in The Plant Cell, 13, 1245, June 2001 "A Role for PsbZ in the Core Complex of Photosystem II" by Nancy A. Eckardt, Editor, News and Reviews. Permission granted by the American Society of Plant Biologists, Copyright 2001.

(Bottom) Courtesy of Peter Nixon (Imperial College London) and Ph.D. thesis of Marko Boehm (2007) "Probing the role of the Band 7 protein superfamily in the cyanobacterium Synechocystis sp. PCC 6803," (Imperial College London).

\section{Sidebar "Molecular Logic"}

Figure 1. Reprinted with permission from S.D Straight, J. Andréasson, G. Kodis, S. Band-yopadhyay, R.H. Mitchell, T.A. Moore, A.L. Moore, and D. Gust, J. Am. Chem. Soc., 127, 9403 (2005); Copyright 2005, American Chemical Society.

\section{Sidebar "Solar Fuels"}

Figure 1. N. S. Lewis and D.G. Nocera, PNAS, 103(43), 15729 (2006); Copyright 2006, National Academy of Sciences, U.S.A.
Figure 2. From K. N. Ferreira, T. M. Iverson, K. Maghlaoui, J. Barber, and S. Iwata, Science, 303, 1831 (2004). Reprinted with permission from AAAS. Inset, courtesy of Daniel Nocera (Massachusetts Institute of Technology).

\section{Sidebar "Metamaterials"}

Figure 1. First "left handed" material photographed by William Padilla (Boston College); Courtesy of David R. Smith (Duke University).

Figure 2. From J.B. Pendry, D. Schurig, and D.R. Smith, Science, 312, 1789 (2006).

Reprinted with permission from AAAS.

\section{CHAPTER 4}

\section{Figure Sources}

Figure 4.1. (Left) Created by Leon Balents (University of California, Santa Barbara) (Right) C.S. Yan, H.K. Mao, W.Li, J. Qian, Y. Zhao, and R.J. Hemley, phys. stat. sol. (a), 201, R25 (2004); Copyright Wiley-VCH Verlag GmbH and Co. KGaA. Reproduced with permission.

Figure 4.2. Courtesy of Ramamoorthy Ramesh, Ying-Hao (Eddie) Chu, Lane W. Martin, and Ms. M. Holcomb (University of California, Berkeley); http://www.mse. berkeley.edu/faculty/ramesh/ramesh.html

Figure 4.3. Reprinted figure $1 \mathrm{~d}$ with permission from T.S. Majumdar, M. Sperl, S. Luding, and R. P. Behringer, Phys. Rev. Lett., 98, 058001 (2007); Copyright 2007, by the American Physical Society.

Figure 4.4. (left) Reprinted by permission from Macmillan Publishers Ltd: Nature, J. Lee, K. Fujita, K. McElroy, J.A. Slezak, M. Wang, Y. Aiura, H. Bando, M. Ishikado, T. Masui, J.-X Zhu, A.V. Balatsky, H. Eisaki, S. Uchida, and J.C. Davis, Nature, 442, 546 (2006); Copyright 2006. 
(right) Reprinted by permission from Macmillan Publishers Ltd: Nature, K.M. Lang, V. Madhavan, J.E. Hoffman, E.W. Hudson, H. Eisaki, S. Ushida, and J.C. Davis, Nature, 415, 412 (2002); Copyright 2002.

Figure 4.5. Reprinted by permission from Macmillan Publishers Ltd: Nature Materials, M. Lee, L. Viciu, L. Li, Y. Wang, M.L. Foo, S. Watauchi, R.A. Pascal Jr, R.J. Cava, and N.P. Ong, Nature Materials, 5, 537 (2006); Copyright 2006.

Figure 4.6. Reprinted from Physica E, J.R. Petta, A.C. Johnson, J.M. Taylor, E.A. Laird, A. Yacoby, M.D. Lukin, C.M. Marcus, M.P. Hanson, A.C. Gossard, 35, 251 (2006); Copyright 2006, with permission from Elsevier.

Figure 4.7. Reprinted Figure 1 with permission from G.C.L. Wong, A. Lin, J.X. Tang, Y. Li, P.A. Janmey, and C.R. Safinya, Phys. Rev. Lett., 91(1), 018103, (2003); Copyright 2003, American Physical Society.

\section{Sidebar "Crystal-Growth Facilities"}

Figure 1. (left panel) Courtesy of Arthur Penn Ramirez (Bell Labs, Alcatel-Lucent) and the Institut for KristallZuchtung, Berlin. (right panel, top image) Courtesy of $\mathrm{M}$. Brian Maple (University of California, San Diego). Published in "US condensed-matter community grapples with availability of crystalline samples" $T$. Feder, Physics Today, 60 (8), 26 (2007). (Right panel, bottom image) Courtesy of Arthur Penn Ramirez (Bell Labs, Alcatel-Lucent) and the Institut for KristallZuchtung.

\section{Sidebar "Frustrated Magnetism"}

Figure 1. Created by Leon Balents (University of California, Santa Barbara).
Figure 2. Reprinted by permission from Macmillan Publishers Ltd: Nature Physics, M. Matsuda, H. Ueda, A. Kikkawa, Y. Tanaka, K. Katsumata, Y. Narumi, T. Inami, Y. Ueda, and S.-H. Lee, Nature Physics, 3, 397 (2007); Copyright 2007.

Figure 3. Reprinted by permission from Macmillan Publishers Ltd: Nature, S.-H. Lee, C. Broholm, W. Ratcliff, G. Gasparovic, Q. Huang, T.H. Kim, and S.-W. Cheong, Nature, 418, 856 (2002); Copyright 2002.

\section{Sidebar "High-Temperature Superconductivity"}

Figure 1. Courtesy of Worawat Meevasana and Z.-X. Shen (Stanford University); http://arpes.stanford.edu/

Figure 2. Reprinted by permission from Macmillan Publishers Ltd: Nature, K. Gomes, A.N. Pasupathy, A. Pushp, S. Ono, Y. Ando, and A. Yazdani, Nature, 447, 569 (2007); Copyright 2007.

\section{Sidebar "Correlated Interfaces"}

Figure 1. Reprinted by permission from Macmillan Publishers Ltd: Nature, A. Ohtomo, D.A. Muller, J.L. Grazul, and H.Y. Hwang, Nature, 419, 378 (2002); Copyright 2002.

Figure 2. Reprinted by permission from Macmillan Publishers Ltd: Nature Materials, A. Brinkman, M. Huijben, M. van Zalk, J. Huijben, U.Zeitler, J.C. Maan, W.G. van der Wiel, G. Rijnders, D.H.A. Blank, and H. Hilgenkamp, Nature Materials, 6, 493 (2007); Copyright 2007.

Figure 3. Reprinted by permission from Macmillan Publishers Ltd: Nature Materials, H. Ohta, S.-W. Kim, Y. Mune, T. Mizoguchi, K. Nomura, S. Ohta, T. Nomura, Y. Nakanishi, Y. Ikuhara, M. Hirano, H. Hosono, and K. Koumoto, Nature Materials, 6, 129 (2007); Copyright 2007. 


\section{Sidebar "Correlated Biological Materials}

Figure 1. (2nd from top) Courtesy of Wouter-Jan Rappel Group (University of California, San Diego); http://physics.ucsd.edu/ $\sim \mathrm{rappel} / \mathrm{research} / \mathrm{res} . \mathrm{html}$

(2nd from bottom) Courtesy of Mark Sussman (San Diego State University Heart Institute); http://heart.sdsu.edu (Bottom) Courtesy of San Diego State University College of Sciences, Biology 590, Human Physiology, Actin Myosin Crossbridge 3D Animation; http://www. sci.sdsu.edu/movies/actin_myosin_gif.html

\section{CHAPTER 5}

\section{Figure Sources}

Figure 5.1. Used with permission, copyright Russell Kightley Media: Scientific Illustration and Animation; http://www.rkm.com.au/

Figure 5.2. Used with permission, copyright Russell Kightley Media: Scientific Illustration and Animation; http://www.rkm.com.au/

Figure 5.3. Reprinted with permission from P. Avouris and J. Appenzeller, Industrial Physicist, 10, 18 (2004); American Institute of Physics.

Figure 5.4. Courtesy of Victor Sourjik (RuprechtKarls-Universität Heidelberg); http:// www. zmbh.uni-heidelberg.de/Sourjik/ default.shtml

Figure 5.5. (left panel) Reprinted by permission from Macmillan Publishers Ltd: Nature, G.E. Murphy, J.R. Leadbetter, and G.J. Jensen, Nature, 442, 1062 (2006); Copyright 2006.

(right panel) Courtesy of Keiichi Namba (Osaka University); http://www.fbs. osaka-u.ac.jp/eng/labo/09a.html
Figure 5.6. Courtesy of Luke Lee (University of California, Berkeley); http://biopoems.openwetware.org/

Figure 5.7. (left panel) Courtesy of S. Park, P.M. Wolanin, E.A. Yuzbashyan, H. Lin, N.C. Darnton, and J.B. Stock, PNAS, 100, 13910 (2003); Copyright 2003, National Academy of Sciences, U.S.A.

(right panel) Courtesy of Steven Miller (Naval Research Laboratory Monterey) and Steve Haddock (Monterey Bay Aquarium Research Institute); http://www.lifesci. ucsb.edu/ biolum/ organism/milkysea.html

\section{Sidebar "Communicating with Cells"}

Figure 1. Created by Raghuveer Parthasarathy (University of Oregon); http://physics. uoregon.edu/ raghu/

Published in J. T. Groves Angew. Chem. Int. Ed. Engl. 44, 3524 (2005). Copyright Wiley-VCH Verlag GmbH and Co. KGaA. Reproduced with permission.

Sidebar "Defectology and the End of Moore's Law"

Figure 1. Courtesy of Greg S. Snider (HewlettPackard) and R. Stanley Williams

(Hewlett-Packard).

\section{Sidebar "Molecular Transport Junctions"}

Figure 1. Courtesy of Martin Chavin et. al, Quantronics group, SPEC CEA-Saclay, France; http://www-drecam.cea.fr/drecam/ spec/Pres/Quantro/

Figure 2. Courtesy of Heike Riel (IBM Zurich Research Laboratory); Earlier version of figure appeared in article by E. Lörtscher, J.W. Ciszek, J. Tour, and H. Riel, Small, 2, (8-9), 973 (2006). 
Figure 3. Reprinted by permission from Macmillan Publishers Ltd: Nature, J. Park, A.N. Pasupathy, J.I. Goldsmith, C. Chang, Y. Yaish, J.R. Petta, M. Rinkoski, J.P. Sethna, H.D. Abruña, P.L. McEuen, and D.C. Ralph, Nature, 417, 722 (2002); Copyright 2002.

Figure 4. Reprinted by permission from Macmillan Publishers Ltd: Nature, J. Park, A.N. Pasupathy, J.I. Goldsmith, C. Chang, Y. Yaish, J.R. Petta, M. Rinkoski, J.P. Sethna, H.D. Abruña, P.L. McEuen, and D.C. Ralph, Nature, 417, 722 (2002); Copyright 2002.

Figure 5. H. S. J. van der Zant, E.A. Osorio, M. Poot, and K. O'Neill, phys. stat. sol. (b), 243, No.13, 3408 (2006). Copyright Wiley-VCH Verlag GmbH and Co. KGaA. Reproduced with permission.

Figure 6. same as above

Figure 7. same as above

\section{Sidebar "Noise Can Be Useful"}

Figure 1. (left) Courtesy of Arup Chakraborty (Massachusetts Institute of Technology); (right) M.N. Artyomov, J. Das, M. Kardar, and A.K. Chakraborty, PNAS, 104, 18958 (2007); Copyright 2007, National Academy of Sciences, U.S.A.

\section{Sidebar "Protein Motors"}

All figures courtesy of George Oster (University of California, Berkeley); http://www.cnr.berkeley.edu/ goster/ home.html

\section{Sidebar "Proticity and Electricity"}

Figure 1. Courtesy of Thomas Moore and Larry Orr (Arizona State University); Reprinted with permission from A.L. Moore, D. Gust, and T. Moore, L'Actualité Chimique May/June 2007.
Figure 2. Courtesy of Thomas Moore and Larry Orr (Arizona State University).

\section{CHAPTER 6}

Figure Sources

Figure 6.1. Stock image

Figure 6.2. Wikipedia; http://en.wikipedia.org/wiki/ Hurricane_Catarina

Figure 6.3. Drawn by Joshua Vura-Weis (Northwestern University).

Figure 6.4. Drawn by Joshua Vura-Weis (Northwestern University).

Figure 6.5. Drawn by Joshua Vura-Weis (Northwestern University).

\section{Sidebar "A Variety of Non-Equilibrium Systems"}

Figure 1. Courtesy of the artist Lino Tagliapietra and Holsten Galleries (Contemporary Glass Sculptures); http://www.holstengalleries.com/gifs/art/tagliapietra/ 608_fenice.jpg

Figure 2. Courtesy of Vladimir K. Vanag and Irving R. Epstein (Brandeis University); V.K. Vanag, and I.R. Epstein, PNAS, 100(25), 14635 (2003); Copyright 2003, National Academy of Sciences, U.S.A.

Figure 3. (top) Photograph by Kristiana KandereGrzybowski; (middle) Courtesy of Eshel Ben-Jacob; both appeared in B.A. Grzybowski, K.J.M. Bishop, C.J. Campbell, M. Fialkowski, and S.K. Smoukov, Soft Matter, 1, 114 (2005); Reproduced by permission of The Royal Society of Chemistry; (bottom) Courtesy of Google images.

Figure 4. Photographs by Bartosz Grzybowski (Northwestern University).

Figure 5. Photograph by Bartosz Grzybowski (Northwestern University). 
Figure 6. Photograph by Bartosz Grzybowski

(Northwestern University).

Figure 7. http://en.wikipedia.org/wiki/Iceland_poppy

Figure 8. Courtesy of Nikonians; http://blog. nikonians.org/archives/MichelleKwan.jpg

\section{Sidebar "Scales of Non-Equilibrium"}

Figures. (Molecular Motor Packing) Reprinted by permission from Macmillan Publishers Ltd: Nature, D. E. Smith, S.J. Tans, S.B. Smith, S.Grimes, D.L. Anderson, and C. Bustamante, Nature, 413, 749, (2001); Copyright 2001.

(All others): stock images.

\section{Sidebar "Nanominerals and Earth Chemistry"}

Figure 1. Reprinted from Geochimica et Cosmochimica Acta, 66, No. 16, 2641 (2007), Copyright 2007, U. Becker, K.M. Rosso, and M.F. Hochella Jr. with permission from Elsevier.

Figure 2. "Aquatic environmental nanoparticles," N. S. Wigginton, K.L. Haus, and M.F. Hochella Jr., Journal of Environmental Monitoring. In Press. Reproduced by permission of the Royal Society of Chemistry.

Figure 3 Courtesy of Michael Hochella (Virginia Tech).

Figure 4. "Aquatic environmental nanoparticles," N. S. Wigginton, K.L. Haus, and M.F. Hochella Jr., Journal of Environmental Monitoring. In Press. Reproduced by permission of the Royal Society of Chemistry.

\section{Sidebar "Thermodynamics of Molecular Machines"}

Figure 1. Courtesy of Jan Liphart and Gavin Crooks (University of California, Berkeley, and Lawrence Berkeley National Laboratory).
Sidebar "Fluctuation Theorems"

Figure 1. Courtesy of Jan Liphart and Gavin Crooks (University of California, Berkeley, and Lawrence Berkeley National Laboratory).

Figure 2. Courtesy of Jan Liphart and Gavin Crooks (University of California, Berkeley, and Lawrence Berkeley National Laboratory).

\section{CHAPTER 7}

\section{Figure Sources}

Figure 7.1. Courtesy of Stephen Jessee, Art Baddorf, and Peter Maksymovych (Center for Nanophase Materials Sciences, Oak Ridge National Laboratory).

Figure 7.2. Reprinted by permission from Macmillan Publishers Ltd: Nature, D.A. Muller, T. Sorsch, S. Moccio, F.H. Baumann, K. Evans-Lutterodt, and G. Timp, Nature, 399, 758 (1999); Copyright 1999.

Figure 7.3. Reprinted by permission from Macmillan Publishers Ltd: Nature, Y. Sugimoto, P. Pou, M. Abe, P. Jelinek, R. Pérez, S. Morita, and Ó. Custance, Nature, 446, 64 (2007); Copyright 2007.

\section{Sidebar "Imaging Molecular Functionality"}

Figure 1. Courtesy of Wilson Ho (University of California, Irvine).

Figure 2. Courtesy of Wilson Ho (University of California, Irvine).

\section{Sidebar "Four Generations of Light Sources"}

Figure 1. Courtesy of John Corlett and Cristoph Steier (Lawrence Berkeley National Laboratory). 


\section{RESOURCE MATERIALS}

In its deliberations, the subcommittee drew on a considerable body of reports and studies, principally from the National Academy of Sciences-National Research Council and the DOE Officer of Basic Energy Sciences. These included:

\section{National Academy of Sciences-National Re- search Council.}

1. Committee on AMO 2010, Controlling the Quantum World: The Science of Atoms, Molecules, and Photons, National Research Council, 2007. Available online at books.nap.edu/catalog. php?record_id=11705.

2. Committee on CMMP 2010, Condensed-Matter and Materials Physics: The Science of the World Around Us, National Research Council, 2007. Available online at books.nap.edu/catalog. php?record_id=11967.

3. Committee on High Energy Density Plasma Physics, Plasma Science Committee, Frontiers in High Energy Density Physics: The X-Games of Contemporary Science, National Research Council, 2003. Available online at books.nap.edu/catalog. php?record_id=10544.

4. Plasma 2010 Committee, Plasma Science: Advancing Knowledge in the National Interest, National Research Council, 2007. Available online at books.nap.edu/catalog.php?record_id=11960.

5. Advancing the Fundamentals of Controlling Quantum Systems, Report of a workshop held at Princeton University, Princeton, NJ, March 18-19, 2005. Report available at www.princeton.edu/ hrabitz/ Quantum\%20Control\%20Workshop/report.htm.

6. Committee on Benchmarking the Research Competitiveness of the United States in Chemistry, The Future of U.S. Chemistry Research. Benchmarks and Challenges, National Research Council, 2007.

Available online at: http://books.nap.edu/ catalog.php?record_id=11866.
7. Keck Futures Initiative Nanoscience and Nanotechnology Planning Committee, Designing Nanostructures at the Interface Between Biomedical and Physical Systems, National Research Council, 2005. Available online at: http://books.nap.edu/catalog. php?record_id=11317.

8. Committee on Globalization of Materials Research and Development, , Globalization of Materials $R E D$ : Time for a National Strategy, National Research Council, 2005. Available online at: http://books.nap.edu/catalog.php?record_id=11395.

9. Committee on Challenges for the Chemical Sciences in the 21st Century, Beyond the Molecular Frontier: Challenges for Chemistry and Chemical Engineering, National Research Council, 2003. Available online at: http://www.nap.edu/ catalog.php?record_id=10633.

10. Committee for the Workshop on Materials and Manufacturing, Committee on Challenges for the Chemical Sciences in the 21st Century, Materials Science and Technology: Challenges for the Chemical Science in the 21st Century, National Research Council, 2003. Available online at: http://books. nap.edu/catalog.php?record_id=10694.

11. Committee on Novel Approaches to the Management of Greenhouse Gases from Energy Systems, Novel Approaches to Carbon Management: Separation, Capture, Sequestration, and Conversion to Useful Products, National Research Council, 2003. Available online at: http://books.nap.edu/catalog. php?record_id=10699

12. Chemical Sciences Roundtable, Board on Chemical Sciences and Technology, Carbon Management: Implications for RED in the Chemical Sciences and Technology, National Research Council,2001. Available online at: http://books.nap.edu/catalog. php?record_id=10153.

\section{Office of Basic Energy Sciences.}

13. Basic Research Needs for Electrical Energy Storage. Report based on a Basic Energy Sciences workshop held April 204, 2007. Available online at: http:// www.sc.doe.gov/bes/reports/files/EES_rpt.pdf. 
14. Basic Research Needs for Geosciences: Facilitating 21st Century Energy Systems. Report based on a BES workshop held February 21-23, 2007. Available online at: http://www.sc.doe.gov/bes/reports/ files/GEO_rpt.pdf.

15. Basic Research Needs for Clean and Efficient Combustion of 21st Century Transportation Fuels. Report based on a Basic Energy Sciences workshop held October 29-November 1, 2006. Available online at: http://www.sc.doe.gov/bes/reports/files/ CTF_rpt.pdf.

16. Basic Research Needs for Advanced Nuclear Energy Systems. Report based on a Basic Energy Sciences workshop held July 31-August 3, 2006. Available online at: http://www.sc.doe.gov/bes/reports/files/ ANES_rpt.pdf.

17. Basic Research Needs for Solid State Lighting. Report based on a Basic Energy Sciences workshop held May 22-24, 2006. Available online at: http:// www.sc.doe.gov/bes/reports/files/SSL_rpt.pdf.

18. Basic Research Needs for Superconductivity. Report based on a Basic Energy Sciences workshop held May 8-10, 2006. Available online at: http:// www.sc.doe.gov/bes/reports/files/SC_rpt.pdf.

19. The Path to Sustainable Nuclear Energy: Basic and Applied Research Opportunities for Advanced Fuel Cycles. Report based on a small DOE-sponsored workshop held September 12-14, 2005. Available online at : http://www.sc.doe.gov/bes/reports/files/ PSNE_rpt.pdf.

20. Basic Research Needs for Solar Energy Utilization. Report based on a Basic Energy Sciences workshop held April 18-21, 2005. Available online at: http://www.sc.doe.gov/bes/reports/files/SEU_ rpt.pdf.

21. Advanced Computational Materials Science: Application to Fusion and Generation IV Fission Reactors. Report is based on a Basic Energy Sciences Workshp held March 31-April 2, 2004. Available online at: http://www.sc.doe.gov/bes/reports/files/ ACMS_rpt.pdf.
22. Subcommittee on Theory and Computation of the Basic Energy Sciences Advisory Committee, $O p$ portunities for Discovery: Theory and Computation in Basic Energy Sciences, U.S. Department of Energy, 2005. Available online at www.sc.doe.gov/bes/ reports/files/OD_rpt.pdf.

23. Nanoscience Research for Energy Needs. Report based on a National Nanotechnology Initiative (NNI) workshop held March 16-18, 2004 and cosponsored by Basic Energy Sciences and the Nanoscale Science, Engineering, and Technology (NSET) subcommittee of the National Science and Technology Council (NSTC). Second edition available online at: http://www.sc.doe.gov/bes/ reports/files/NREN_rpt.pdf.

24. DOE-NSF-NIH Workshop on Opportunities in THz Science. Report based on a workshop jointly sponsored by DOE, NSF, and NIH held February 1214, 2004. Available online at: http://www.sc.doe. gov/bes/reports/files/THz_rpt.pdf.

25. Basic Research Needs for the Hydrogen Economy. Report based on the Basic Energy Sciences workshop held May 13-15, 2003. Available online at: http://www.sc.doe.gov/bes/reports/files/NHE_rpt. pdf.

26. Theory and Modeling in Nanoscience. Report based on a work held May 10-11, 2002 and conducted jointly by the Basic Energy Sciences Advisory Committee and the Advanced Scientific Computing Advisory Committees. Available at: http:// www.sc.doe.gov/bes/reports/files/TMN_rpt.pdf/

27. Opportunities for Catalysis in the 21st Century. Report based upon a Basic Energy Sciences Advisory Committee subpanel workshop held May 14-16, 2002. Available at: http://www.sc.doe.gov/bes/ reports/files/OC_rpt.pdf.

28. Biomolecular Materials. Report based on a Basic Energy Sciences Advisory Committee workshop held January 13-15, 2002. Available at: http:// www.sc.doe.gov/bes/reports/files/BM_rpt.pdf. 
29. Basic Research Needs to Assure a Secure Energy Future. Report based on a Basic Energy Sciences Advisory Committee workshop held in October 2002. Available online at: http://www.sc.doe.gov/bes/ reports/files/SEF_rpt.pdf.

30. Basic Research Needs to Counter Terrorism. Report based on a Basic Energy Sciences workshop held on February 28 to March 1, 2002. Available online at: http://www.sc.doe.gov/bes/reports/files/ NCT_rpt.pdf.
31. Complex Systems: Science for the 21st Century. Report based on a Basic Energy Sciences workshop held March 5-6, 1999. Available online at: http://www.sc.doe.gov/bes/reports/files/NCT_rpt. pdf.

32. Nanoscale Science, Engineering and Technology Research Directions. Report prepared in 1999 by the Basic Energy Sciences Nanoscience/Nanotechnology Group. Available online at: http://www.sc.doe. gov/bes/reports/files/NSET_rpt.pdf. 


\section{DISCLAIMER}

This report was prepared as an account of work sponsored by an agency of the United States Government. Neither the United States government nor any agency thereof, nor any of their employees, makes any warranty, express or implied, or assumes any legal liability or responsibility for the accuracy, completeness, or usefulness of any information, apparatus, product, or process disclosed, or represents that its use would not infringe privately owned rights. Reference herein to any specific commercial product, process, or service by trade name, trademark, manufacturer, or otherwise, does not necessarily constitute or imply its endorsement, recommendation, or favoring by the United States Government or any agency thereof. The views and opinions of authors expressed herein do not necessarily state or reflect those of the United States Government or any agency thereof.

Report available online at http://www.sc.doe.gov/bes/reports/list.html.

Report prepared at Lawrence Berkeley National Laboratory

Editor: Arthur L. Robinson (Advanced Light Source Division)

Writer: Lynn Yarris (Communications Department)

Design, production, and printing: Creative Services Office (Alice Ramirez, Flavio Robles, Jr., Michael Muller, Caitlin Youngquist, Faye Jobes, Cheryl Ventimiglia)

CSO_14212 

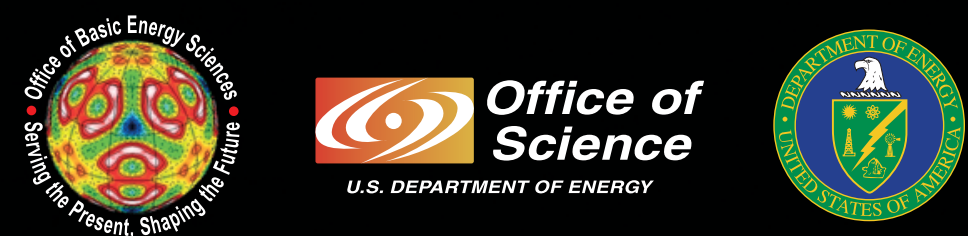